\title{
TfOH-Catalyzed Phosphinylation of 2,3-Allenols into $\gamma$-Ketophosphine Oxides
}

\author{
Runmin Zhao, $\uparrow$ Xianhua Huang, $\uparrow$ Minning Wang, $\neq$ Shanshan Hu, $\neq$ Yuxing Gao, $\neq^{*}$ Pengxiang Xu $t^{*}$ and Yufen \\ Zhaof \\ Fepartment of Chemical and Biochemical Engineering, College of Chemistry and Chemical Engineering, \\ Xiamen University, Xiamen 361005, Fujian, China. \\ $\neq$ Department of Chemistry and Key Laboratory for Chemical Biology of Fujian Province, College of Chemistry \\ and Chemical Engineering, Xiamen University, Xiamen 361005, Fujian, China. \\ *E-mail: gaoxingchem@xmu.edu.cn; xpengxiang@xmu.edu.cn.
}

\section{Table of Contents}

Page S2-S3 1. Crystallographic detail of $\mathbf{3 e}$

Page S4-S67 2. $\quad{ }^{31} \mathrm{P}$ NMR, ${ }^{1} \mathrm{H}$ NMR, ${ }^{13} \mathrm{C}$ NMR Spectra of Compounds 3a-3ap, 4 and 5 


\section{Crystallographic detail of $3 \mathrm{e}$}

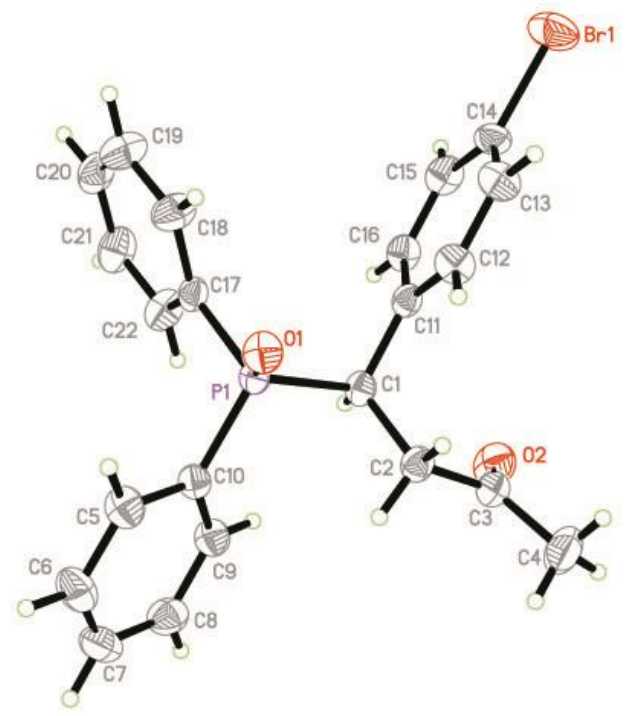

The single crystal of compound 3e was prepared from its solution in acetone by slow evaporation at room temperature. Single crystal X-ray structure of compound 3e, showing 50\% probability displacement ellipsoids (arbitrary spheres for $\mathrm{H}$ atoms). Compound $\mathbf{3 e}$ crystallizes in the monoclinic P -1 space group with two 3 e per unit cell. There is one independent molecule in the asymmetric unit of $\mathbf{3 e}$.

Detector with graphite-monochromated CuKa radiation $(\lambda=0.71073 \AA)$ at $273 \mathrm{~K}$. All of the Data were corrected for absorption effects using the multi-scan technique. The structures were solved by direct methods using OLEX2 (Version 1.2.7) program package. Non-H atoms were refined anisotropically unless otherwise stated. Hydrogen atoms were introduced at their geometric positions and refined as riding atoms unless otherwise stated. Crystal data and details on data collection and refinement are summarized in Table S1. Further details on the crystal structure investigation have been deposited at the Cambridge Crystallographic Data Centre as the deposition number CCDC 1982767. 
Table S1. Crystallographic data and structure refinement parameters for compound $\mathbf{3 e .}$

\begin{tabular}{|l|l|}
\hline Identification code & $\mathrm{CCDC} 1982767$ \\
\hline Empirical formula & $\mathrm{C}_{22} \mathrm{H}_{20} \mathrm{BrO}_{2} \mathrm{P}$ \\
\hline Formula weight & 427.25 \\
\hline Temperature/K & 173 \\
\hline Crystal system & monoclinic \\
\hline Space group & $\mathrm{P}-1$ \\
\hline $\mathrm{a} / \AA$ & $5.6728(12)$ \\
\hline $\mathrm{b} / \AA$ & $9.823(2)$ \\
\hline $\mathrm{c} / \AA$ & $17.884(4)$ \\
\hline$\alpha /{ }^{\circ}$ & $86.194(3)$ \\
\hline$\beta /{ }^{\circ}$ & $88.953(4)$ \\
\hline$\gamma /{ }^{\circ}$ & $75.743(3)$ \\
\hline Volume/ $\AA^{3}$ & $963.7(4)$ \\
\hline $\mathrm{Z}$ & 2 \\
\hline$\rho_{\text {calc }} / \mathrm{cm}^{3}$ & 1.472 \\
\hline $\mathrm{F}(000)$ & 436.0 \\
\hline Final R indices $[\mathrm{I}>2 \sigma(\mathrm{I})]$ & $\mathrm{R}_{1}=0.0576, \mathrm{wR}_{2}=0.1540$ \\
\hline
\end{tabular}


2. ${ }^{1} \mathrm{H},{ }^{13} \mathrm{C}$ and ${ }^{31} \mathrm{P}$ NMR spectra of 3a-3an, 4 and 5

${ }^{1} \mathrm{H} \mathrm{NMR}\left(\mathrm{CDCl}_{3}, 500 \mathrm{MHz}\right)$ of $\mathbf{3 a}$ :

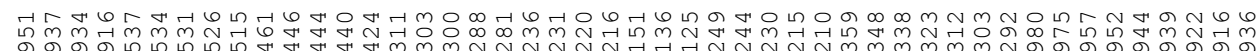

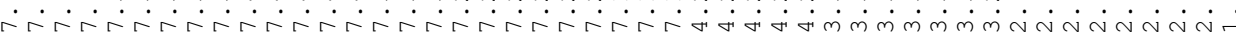

$\rightarrow$
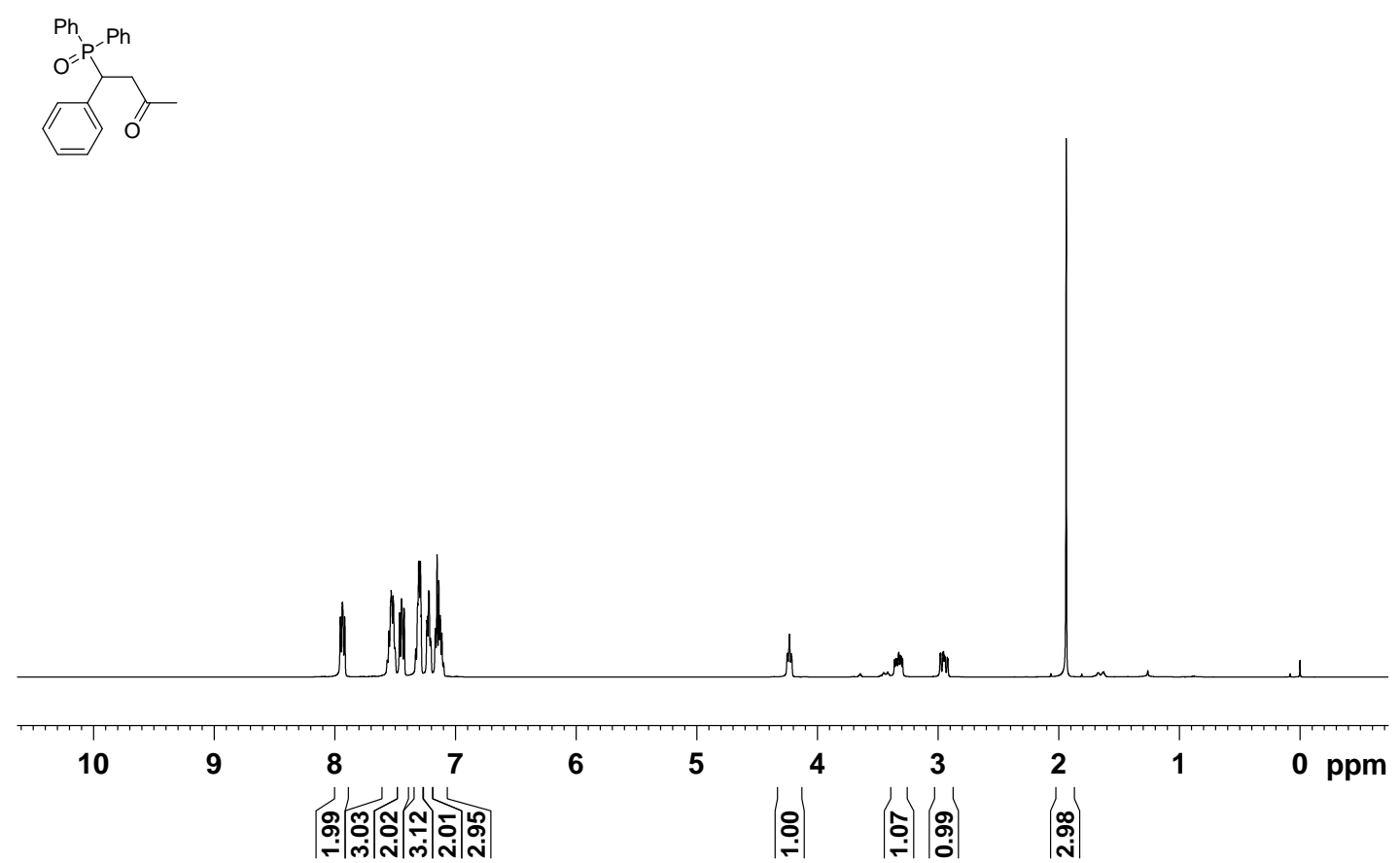

${ }^{13} \mathrm{C}$ NMR $\left(\mathrm{CDCl}_{3}, 125 \mathrm{MHz}\right)$ of $\mathbf{3 a}$ :

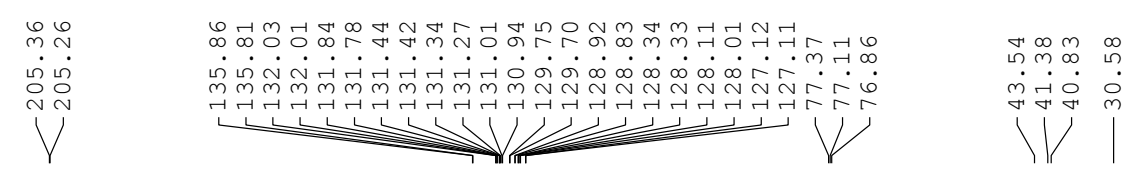

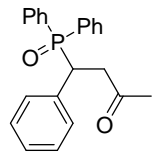

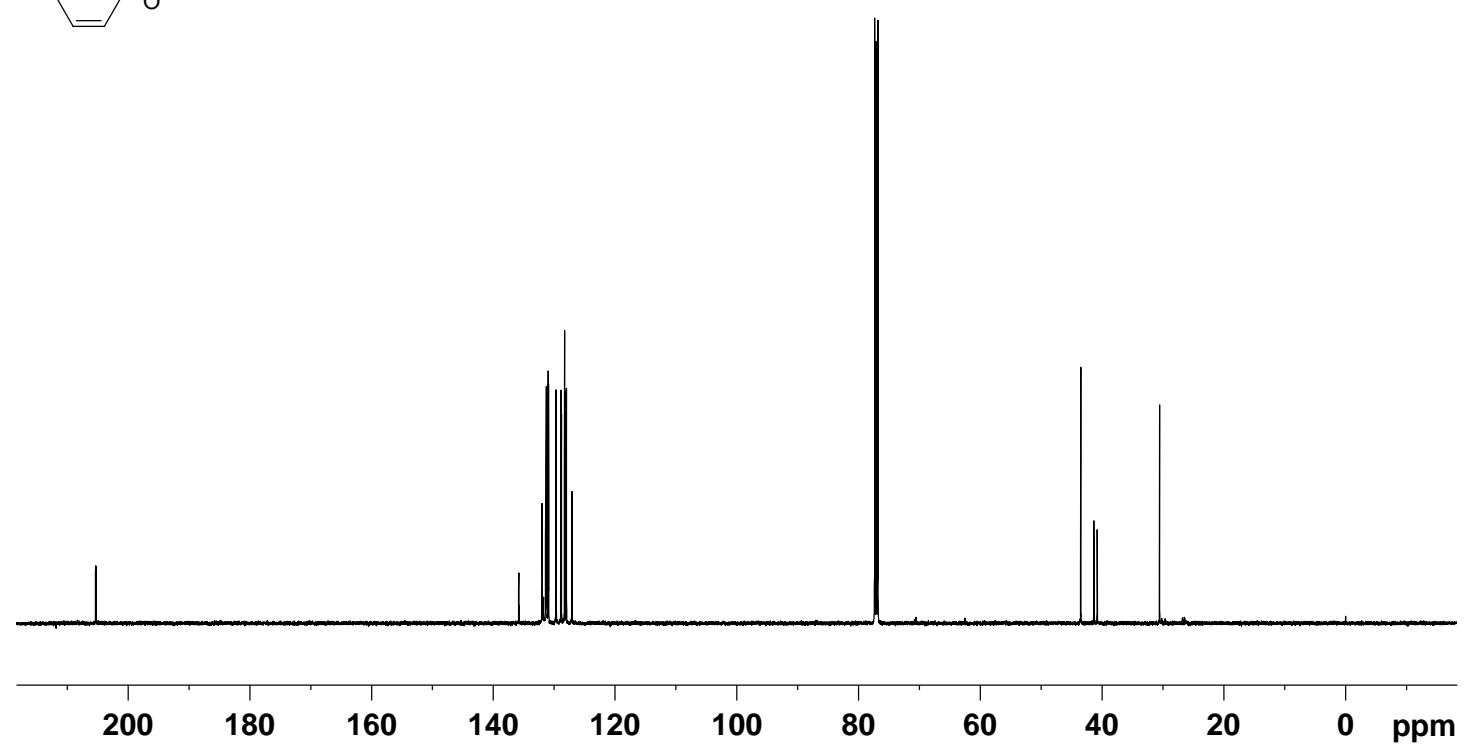


${ }^{31} \mathrm{P} \mathrm{NMR}\left(\mathrm{CDCl}_{3}, 202 \mathrm{MHz}\right)$ of $\mathbf{3 a}$ :

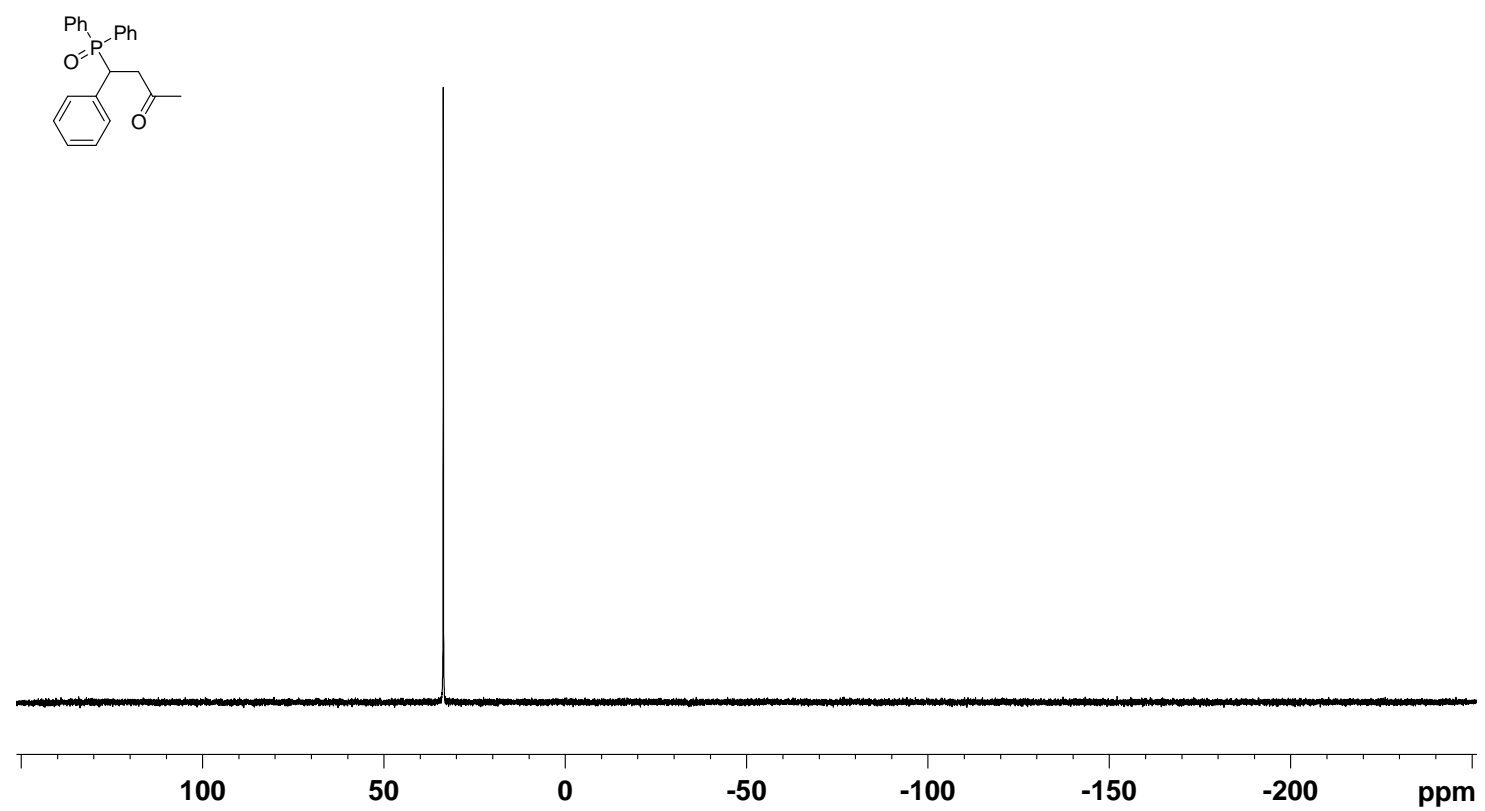

${ }^{1} \mathrm{H} \mathrm{NMR}\left(\mathrm{CDCl}_{3}, 500 \mathrm{MHz}\right)$ of $\mathbf{3 b}$ :

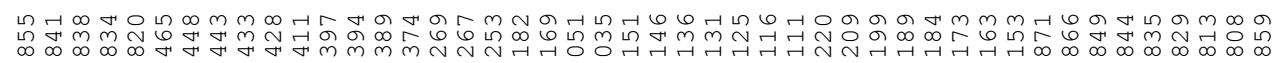

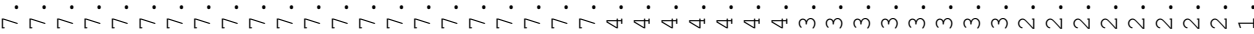

(n)
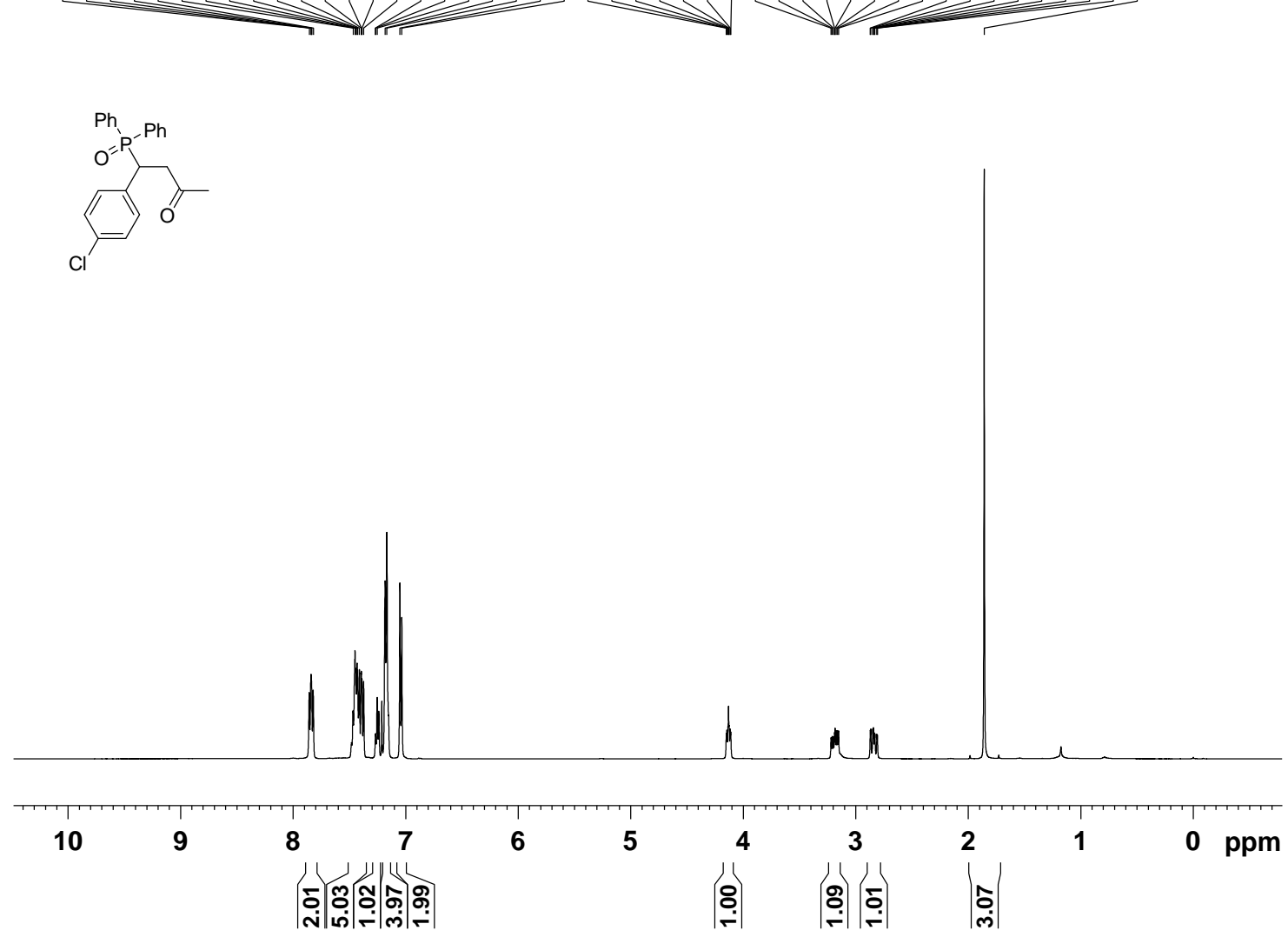
${ }^{13} \mathrm{C}$ NMR $\left(\mathrm{CDCl}_{3}, 125 \mathrm{MHz}\right)$ of $\mathbf{3 b}$ :
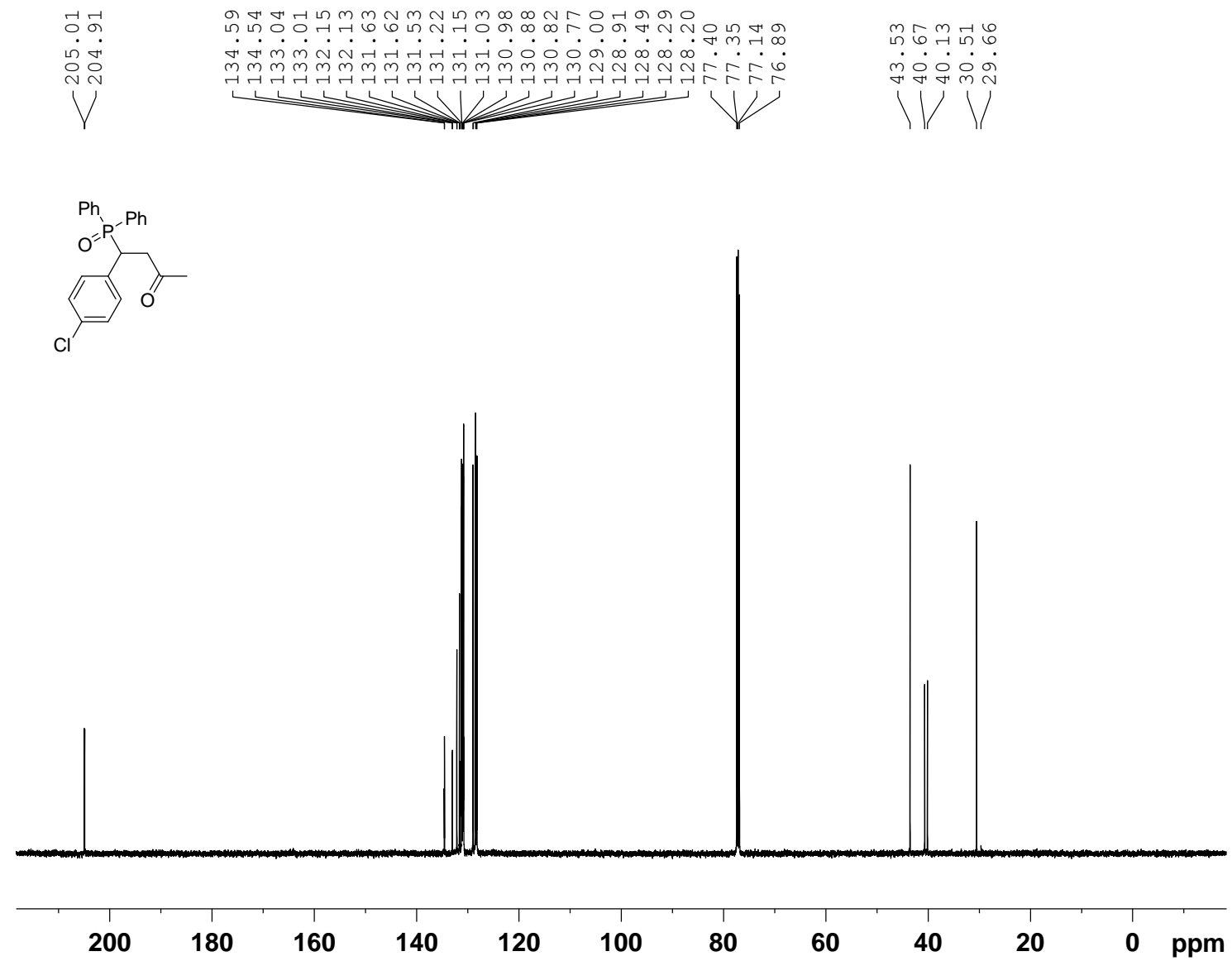

${ }^{31} \mathrm{P} \mathrm{NMR}\left(\mathrm{CDCl}_{3}, 202 \mathrm{MHz}\right)$ of $\mathbf{3 b}$ :
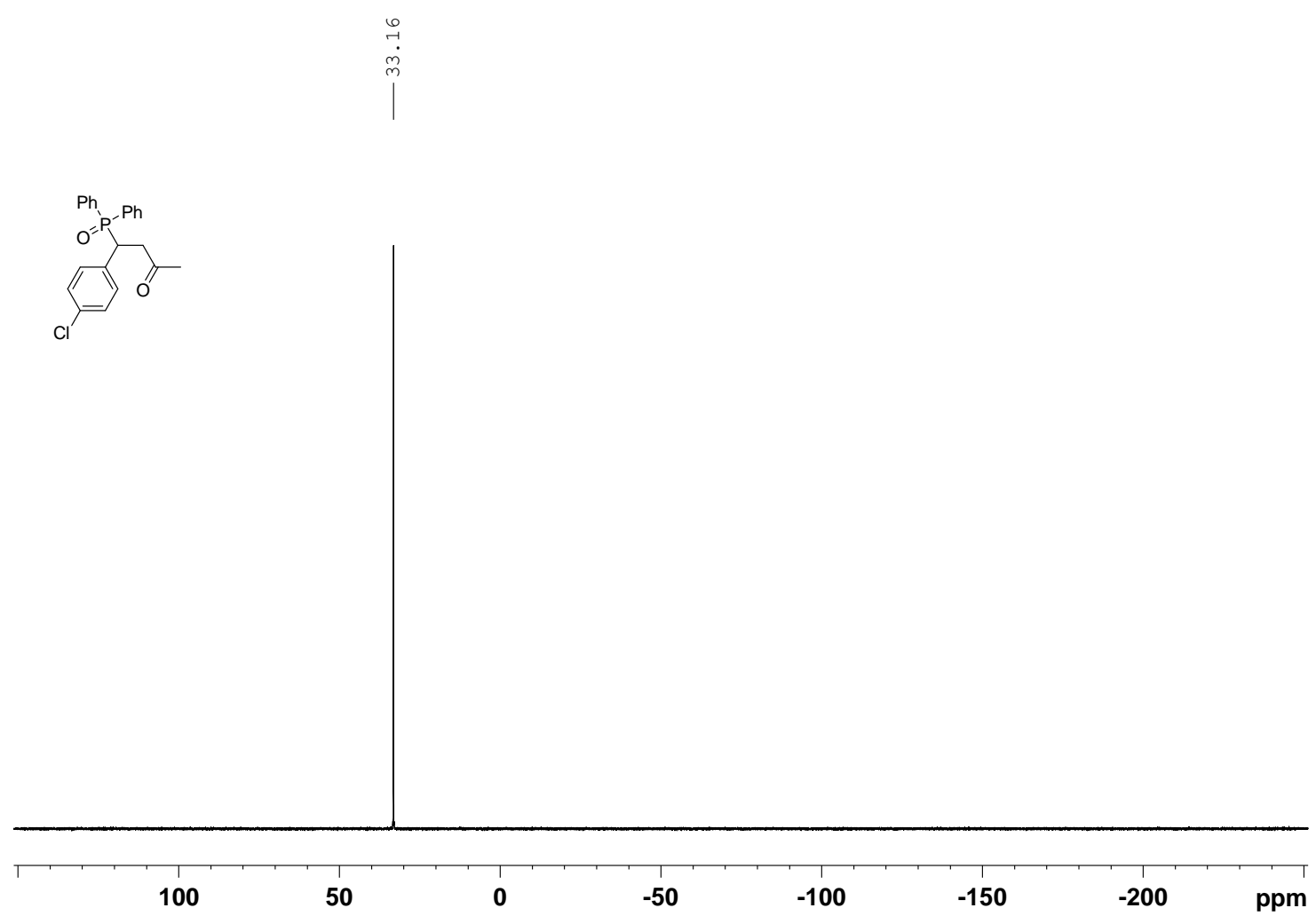
${ }^{1} \mathrm{H} \mathrm{NMR}\left(\mathrm{CDCl}_{3}, 500 \mathrm{MHz}\right)$ of $\mathbf{3 c}$ :

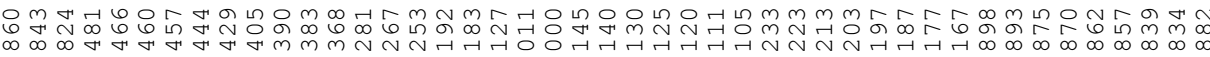

-

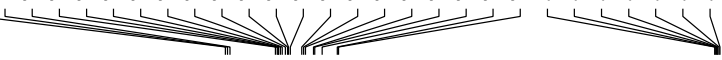

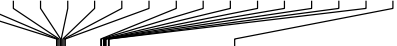

Cl

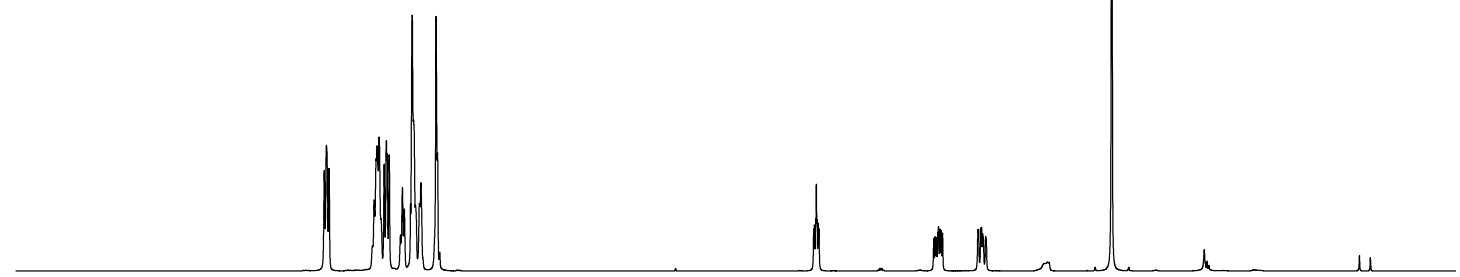

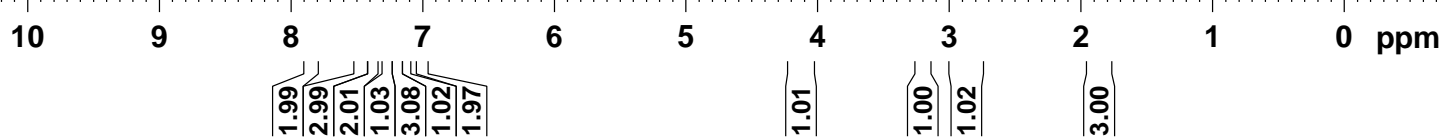

${ }^{13} \mathrm{C} \mathrm{NMR}\left(\mathrm{CDCl}_{3}, 125 \mathrm{MHz}\right)$ of $\mathbf{3 c}$ :
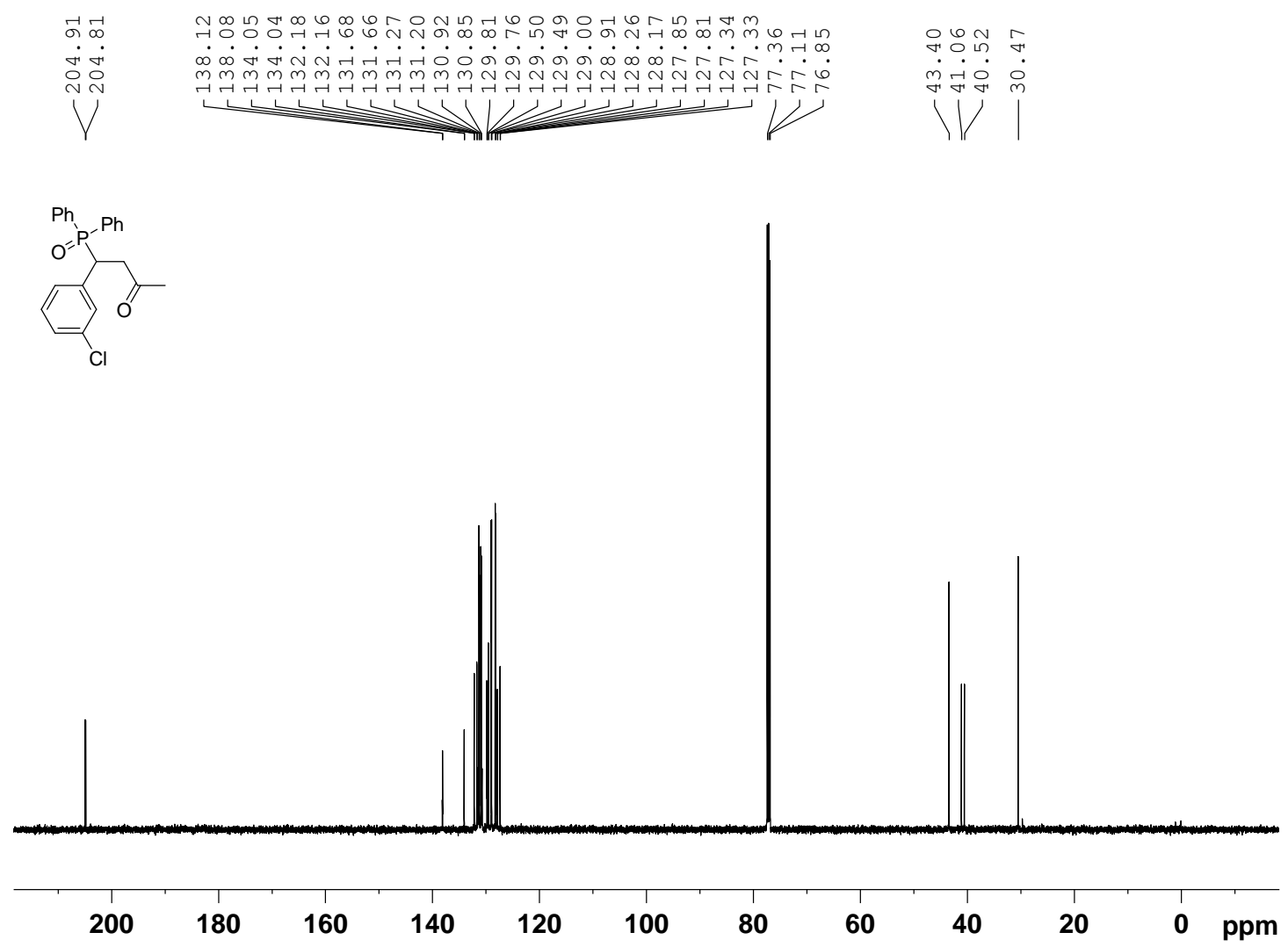
${ }^{31} \mathrm{P}$ NMR $\left(\mathrm{CDCl}_{3}, 202 \mathrm{MHz}\right)$ of $\mathbf{3 c}$ :

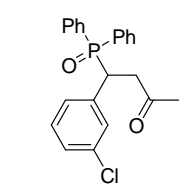

100

50

$-50$

$-100$

$-150$

$-200$

ppm

\section{${ }^{1} \mathrm{H} \mathrm{NMR}\left(\mathrm{CDCl}_{3}, 500 \mathrm{MHz}\right)$ of $\mathbf{3 d}$ :}

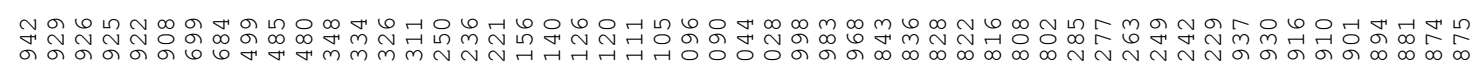

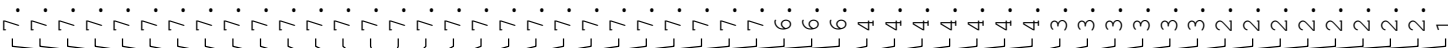

$=0$

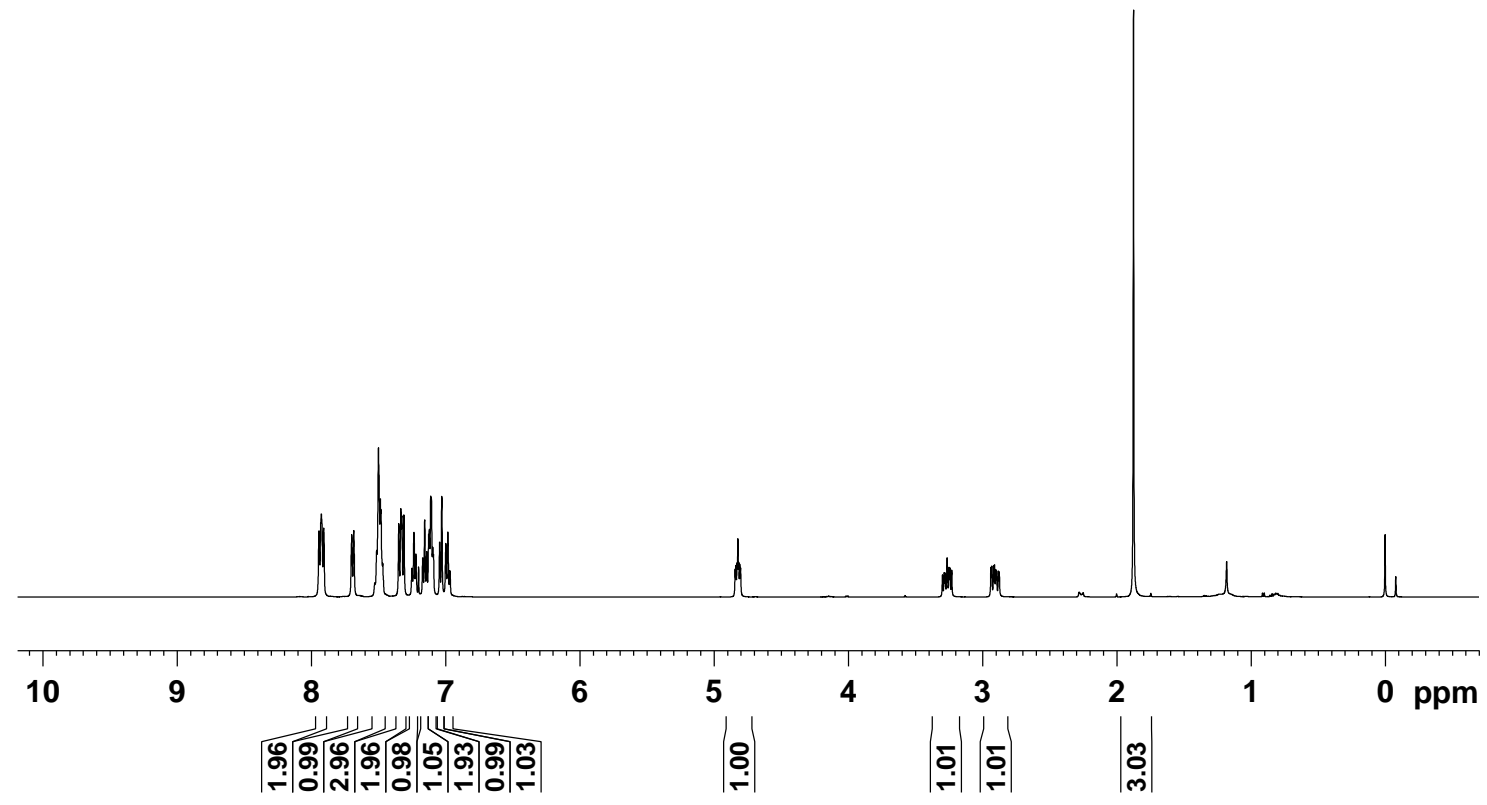


${ }^{13} \mathrm{C}$ NMR $\left(\mathrm{CDCl}_{3}, 125 \mathrm{MHz}\right)$ of $\mathbf{3 d}$ :

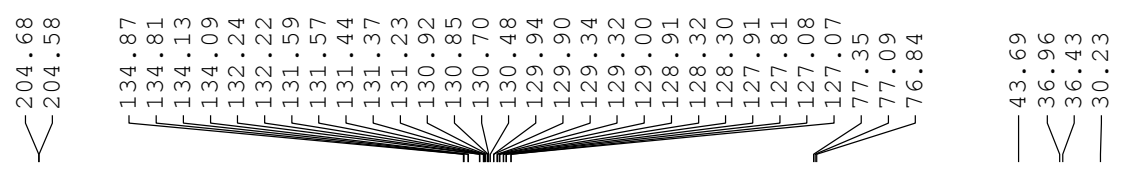

(1)

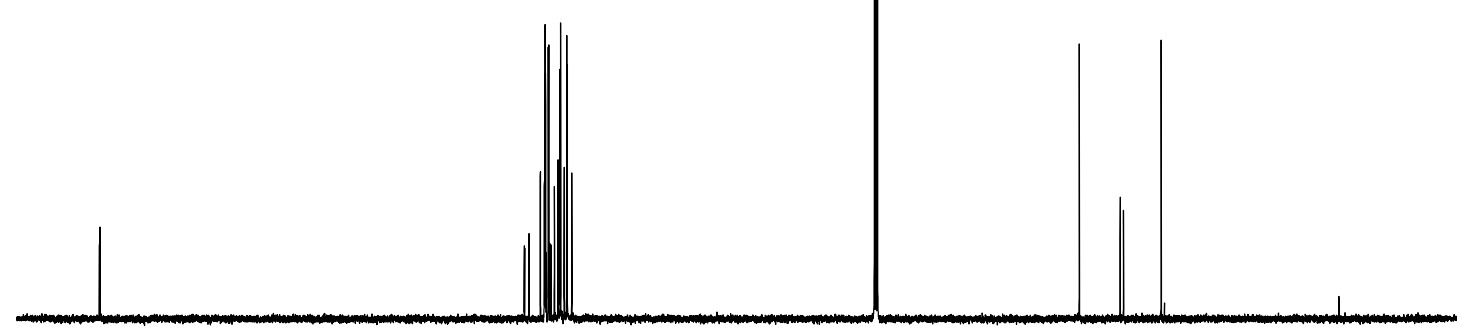

200

$\begin{array}{lll}180 & 160 \quad 140\end{array}$

120

100

80

60

40

${ }^{31} \mathrm{P}$ NMR $\left(\mathrm{CDCl}_{3}, 202 \mathrm{MHz}\right)$ of $\mathbf{3 d}$ :

年)

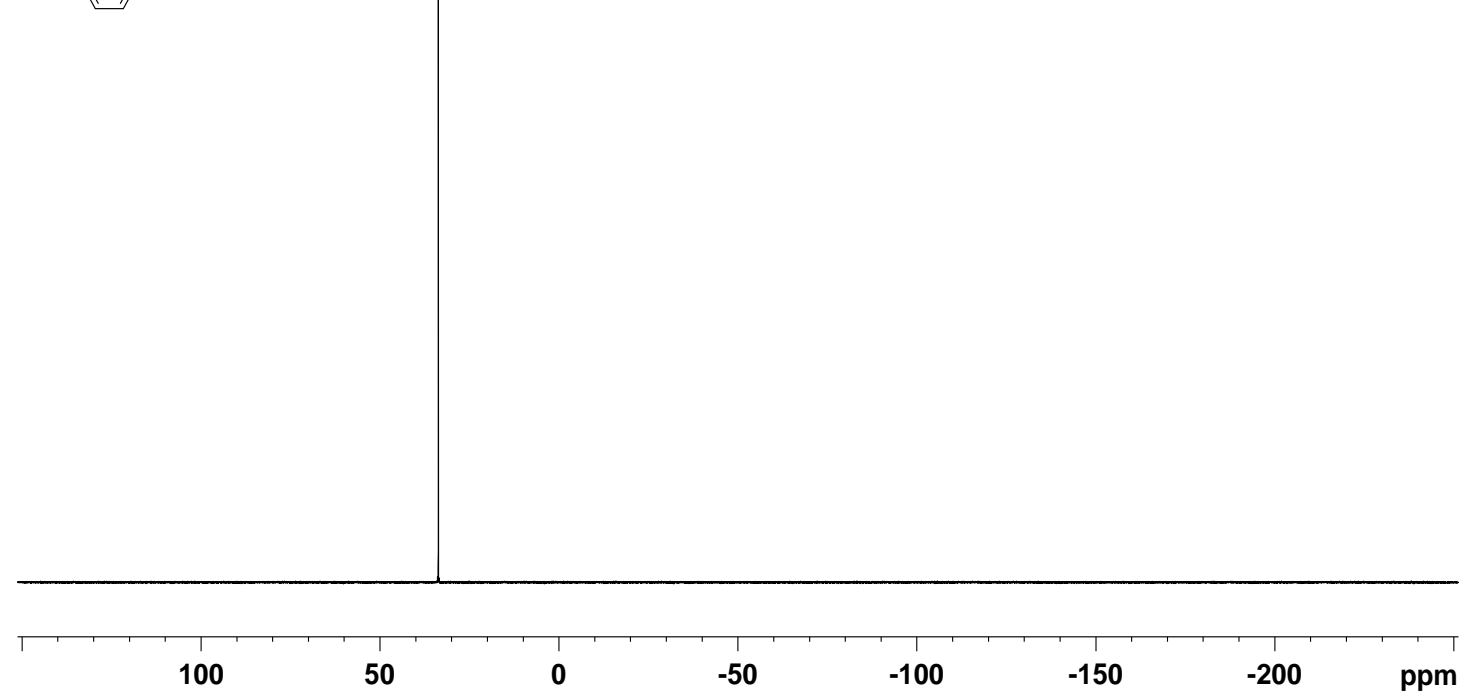


${ }^{1} \mathrm{H} \mathrm{NMR}\left(\mathrm{CDCl}_{3}, 500 \mathrm{MHz}\right)$ of $3 \mathrm{e}:$

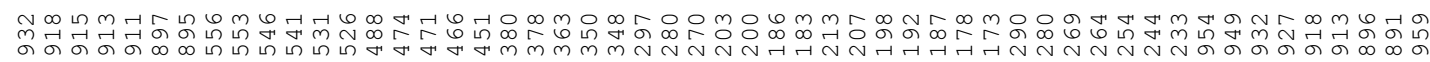

-

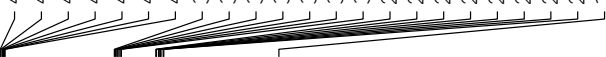

(n)

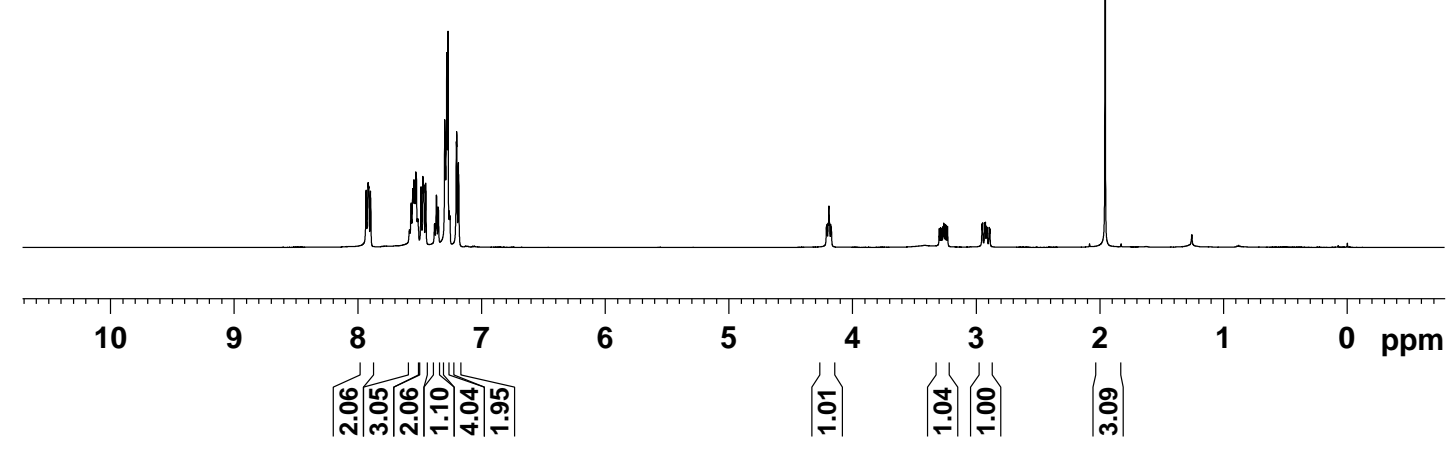

${ }^{13} \mathrm{C} \mathrm{NMR}\left(\mathrm{CDCl}_{3}, 125 \mathrm{MHz}\right)$ of $\mathbf{3 e}:$
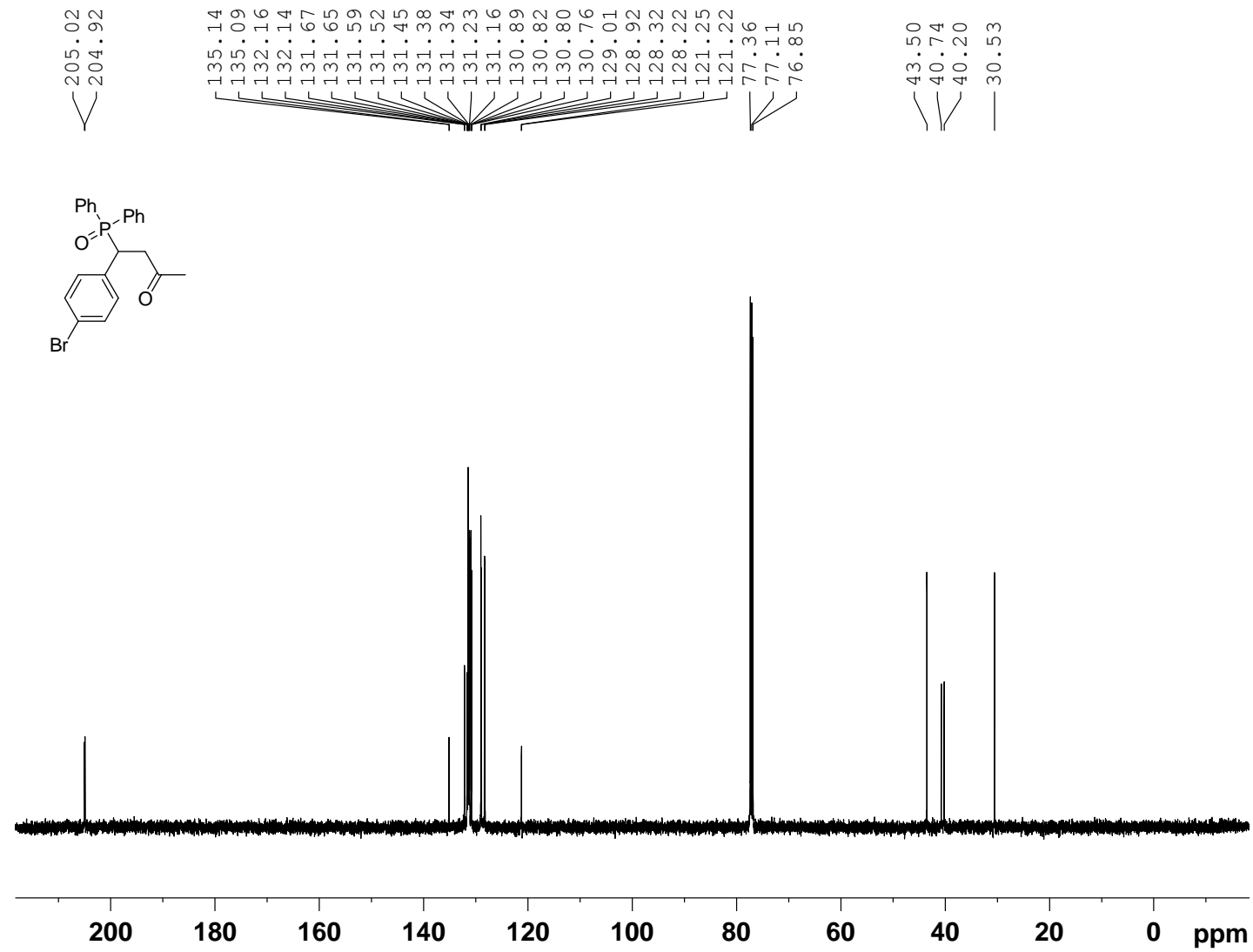
${ }^{31} \mathrm{P}$ NMR $\left(\mathrm{CDCl}_{3}, 202 \mathrm{MHz}\right)$ of $\mathbf{3 e}$ :<smiles>CC(=O)CC(c1ccc(Br)cc1)P(=O)(c1ccccc1)c1ccccc1</smiles>

${ }^{1} \mathrm{H}$ NMR $\left(\mathrm{CDCl}_{3}, 500 \mathrm{MHz}\right)$ of $\mathbf{3 f}$ :

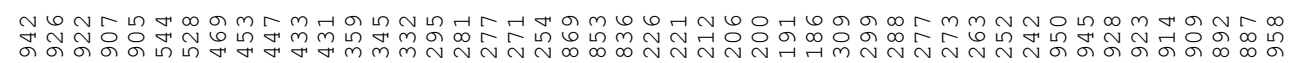

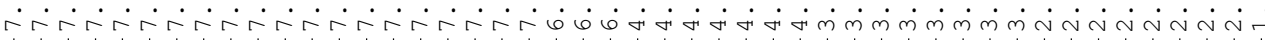

N

(O)

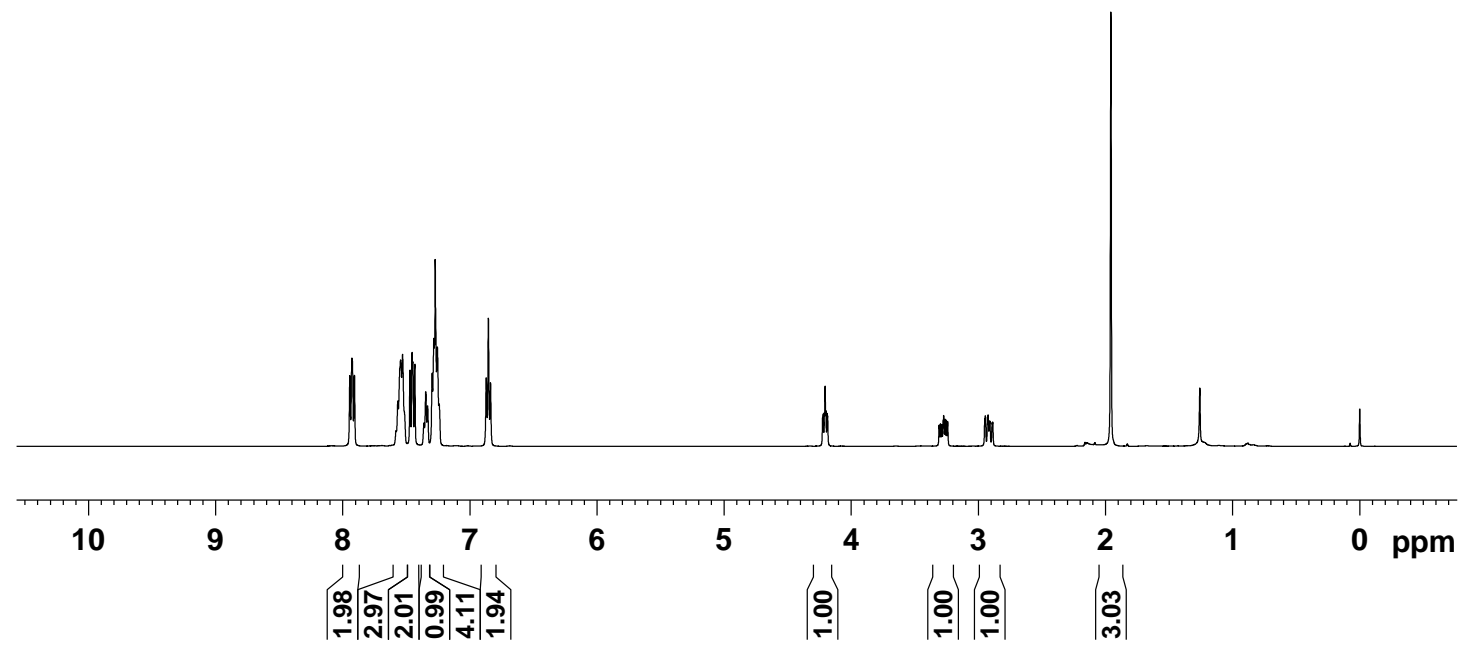


${ }^{13} \mathrm{C} \mathrm{NMR}\left(\mathrm{CDCl}_{3}, 125 \mathrm{MHz}\right)$ of $\mathbf{3 f}$ :
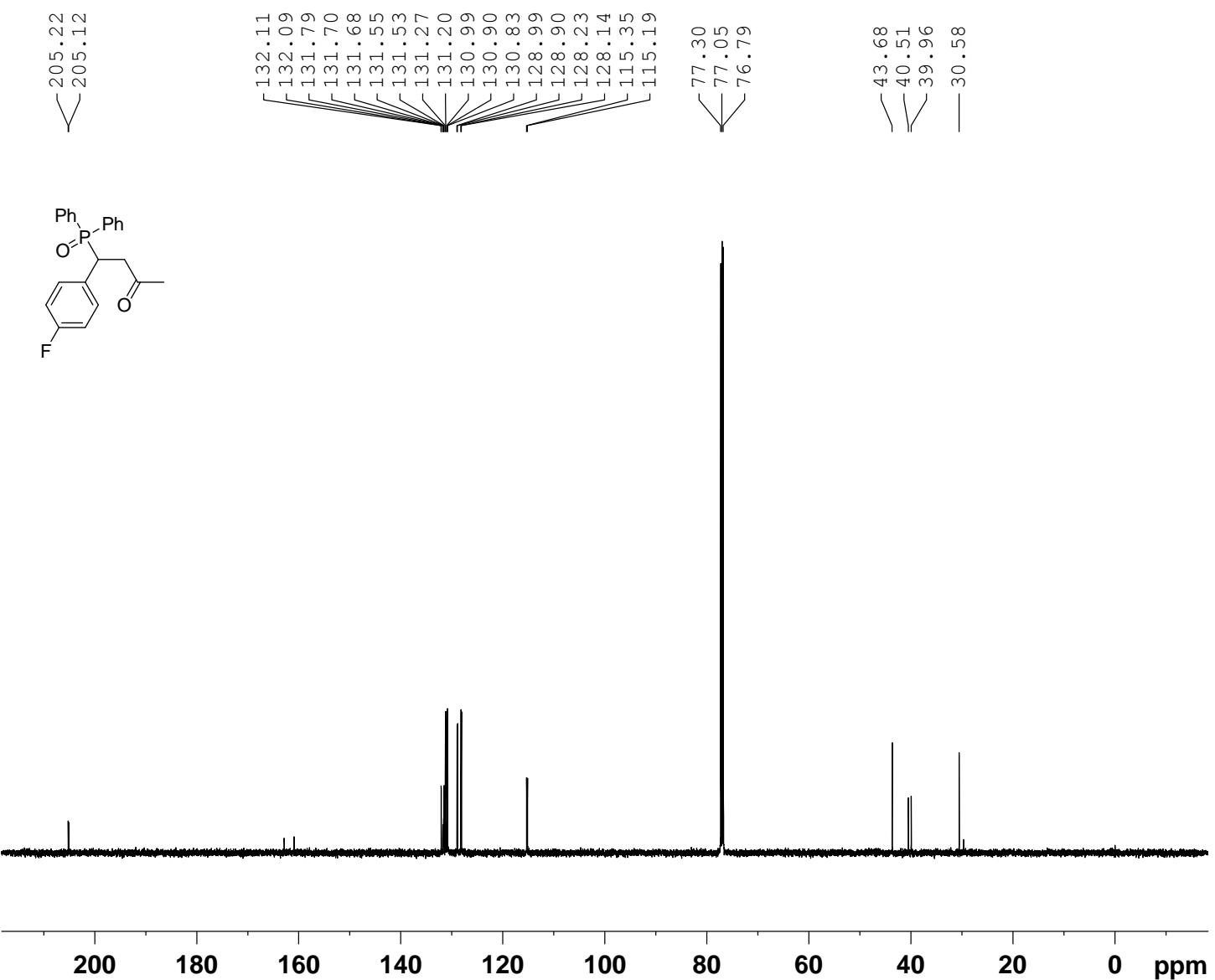

${ }^{31} \mathrm{P} \mathrm{NMR}\left(\mathrm{CDCl}_{3}, 202 \mathrm{MHz}\right)$ of $\mathbf{3 f}$ :
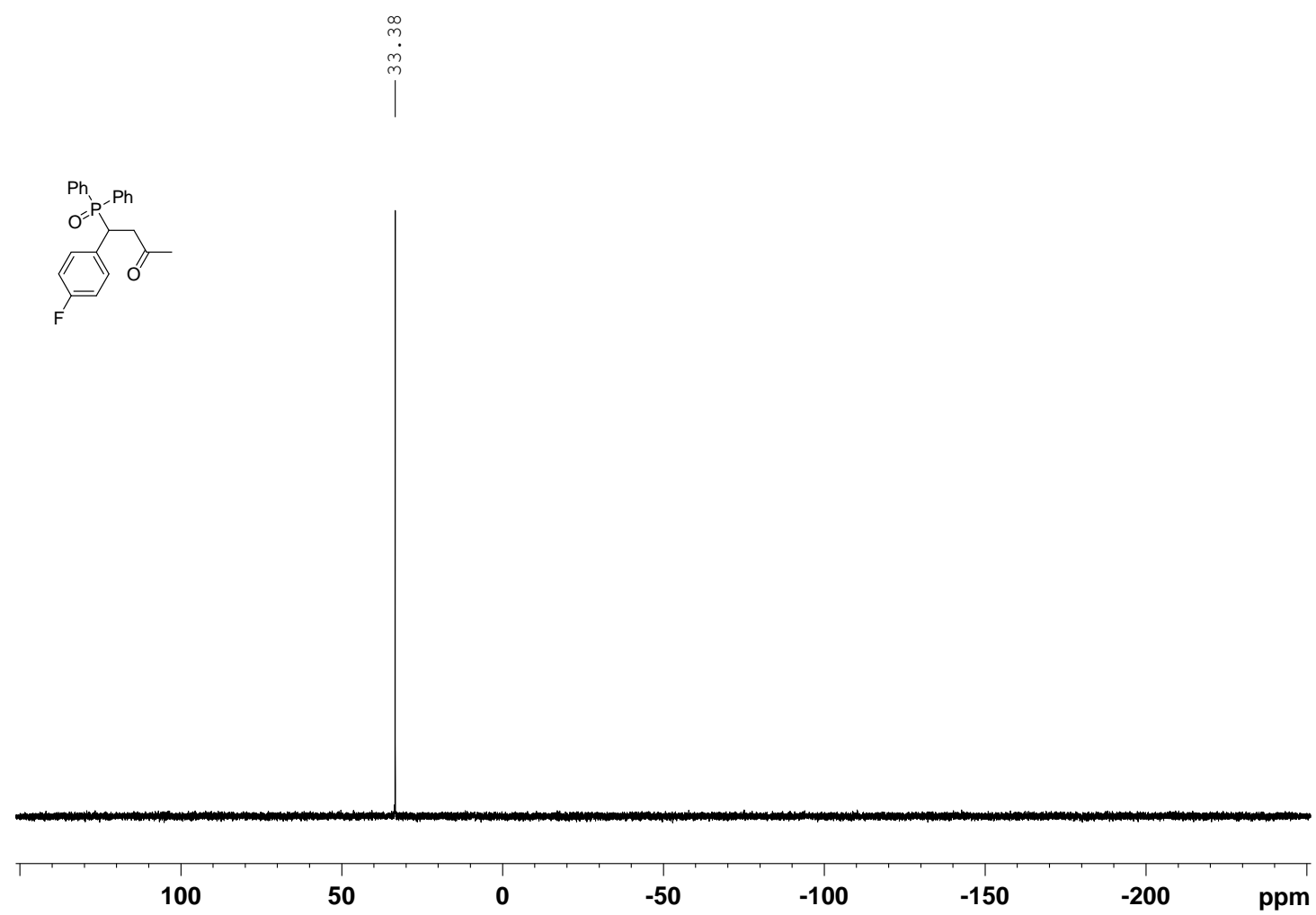
${ }^{1} \mathrm{H} \mathrm{NMR}\left(\mathrm{CDCl}_{3}, 500 \mathrm{MHz}\right)$ of $\mathbf{3 g}$ :

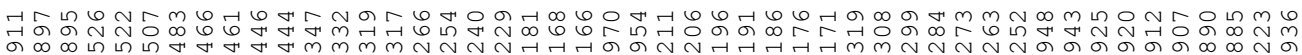

-

(P)

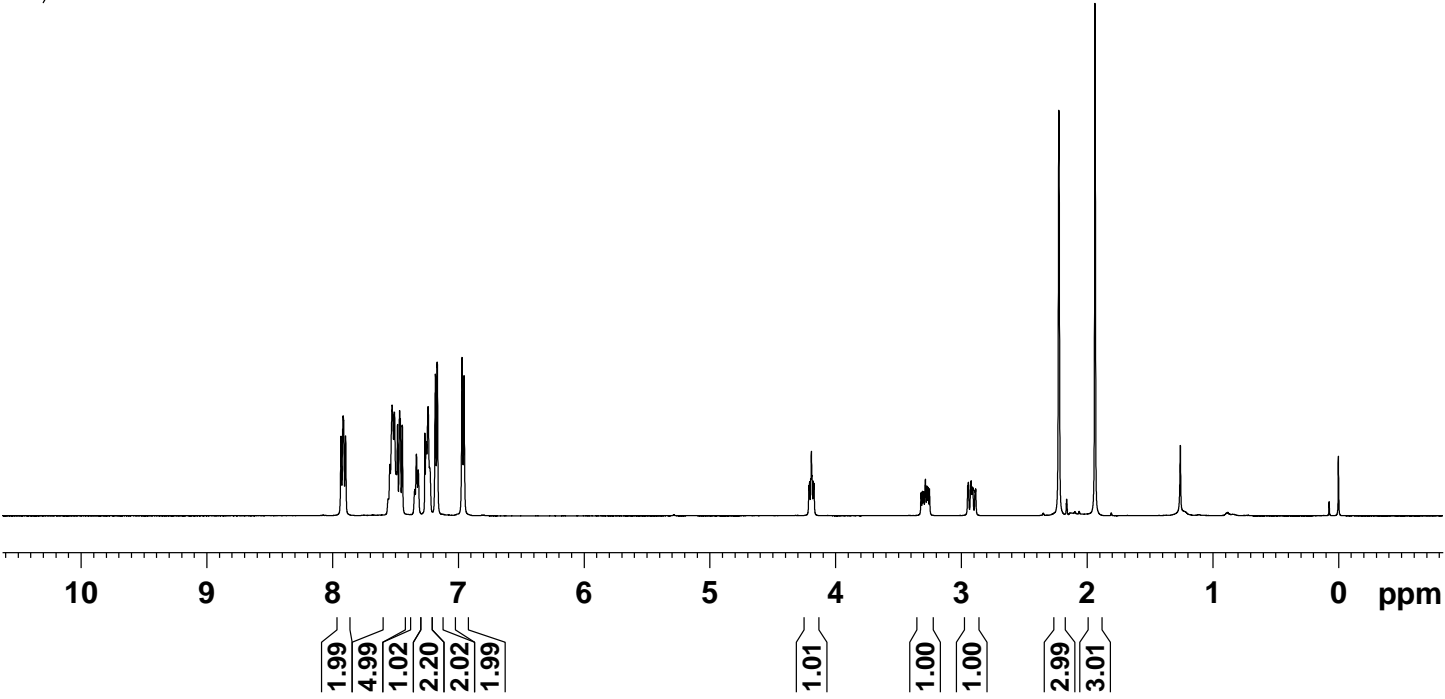

${ }^{13} \mathrm{C} \mathrm{NMR}\left(\mathrm{CDCl}_{3}, 125 \mathrm{MHz}\right)$ of $\mathbf{3 g}$ :

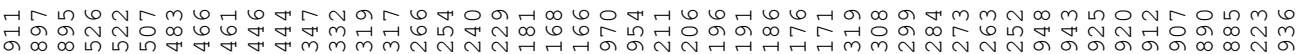

-

$+1$

(1)

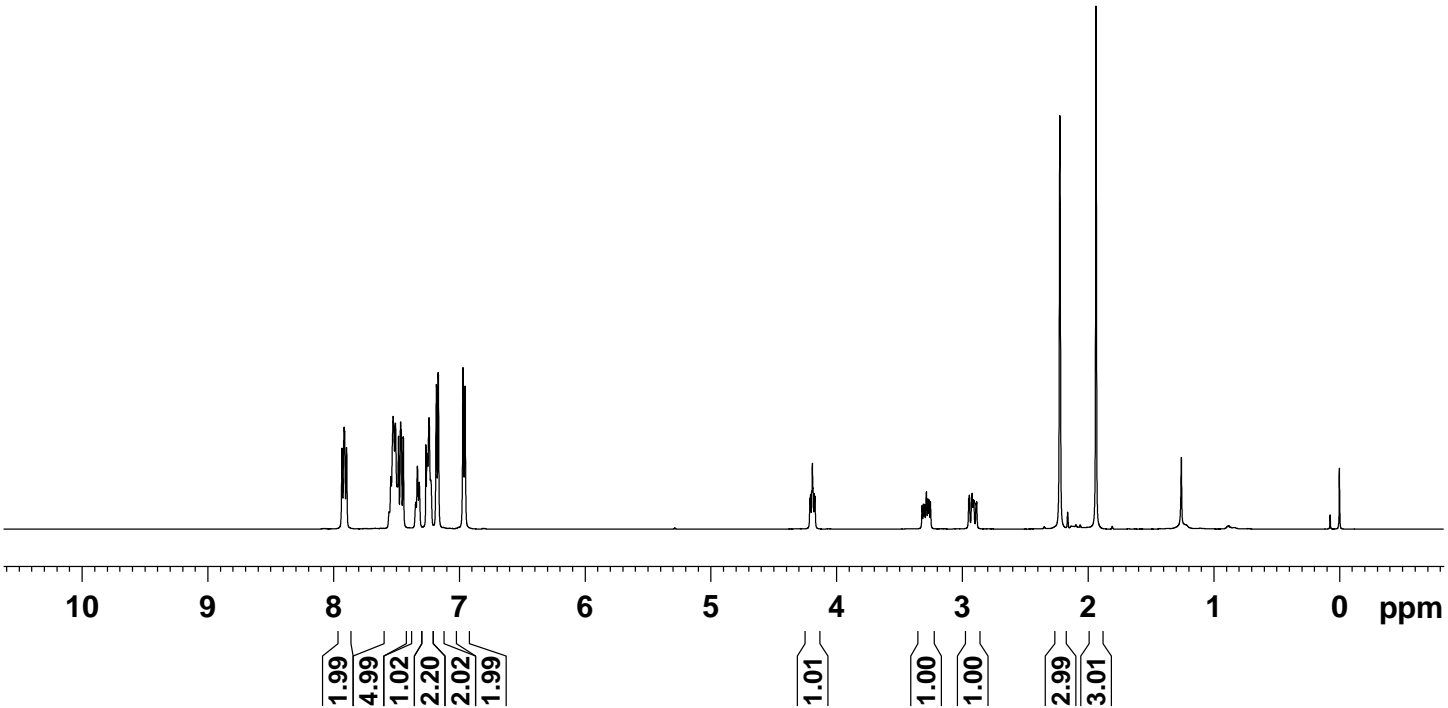


${ }^{31} \mathrm{P} \mathrm{NMR}\left(\mathrm{CDCl}_{3}, 202 \mathrm{MHz}\right)$ of $\mathbf{3 g}$ :
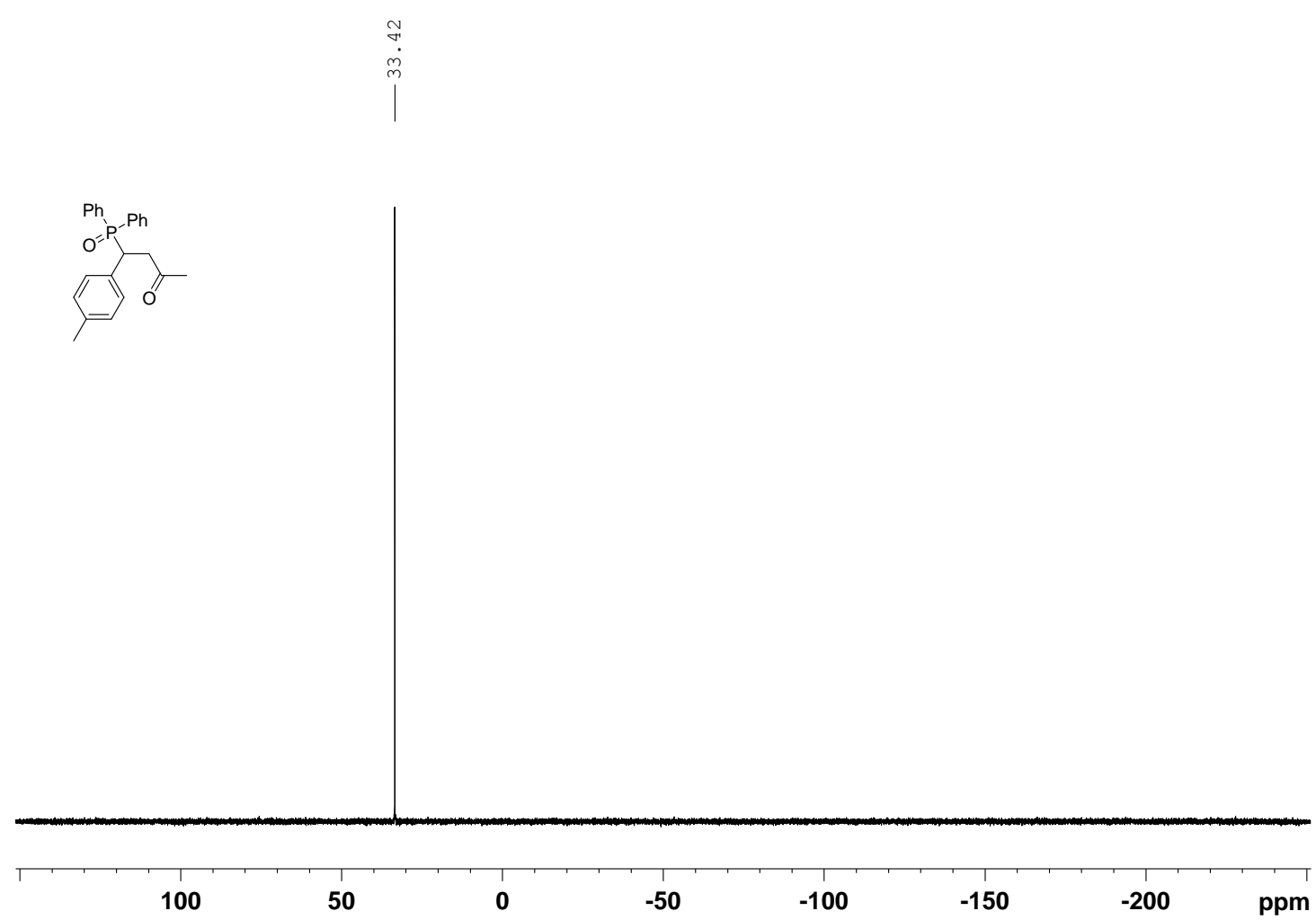

${ }^{1} \mathrm{H} \mathrm{NMR}\left(\mathrm{CDCl}_{3}, 500 \mathrm{MHz}\right)$ of $\mathbf{3 h}$ :

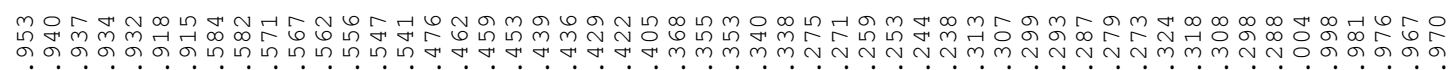

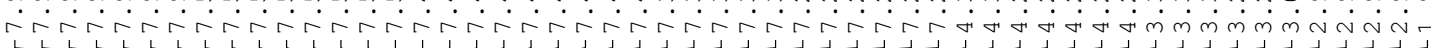

(1)

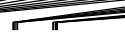

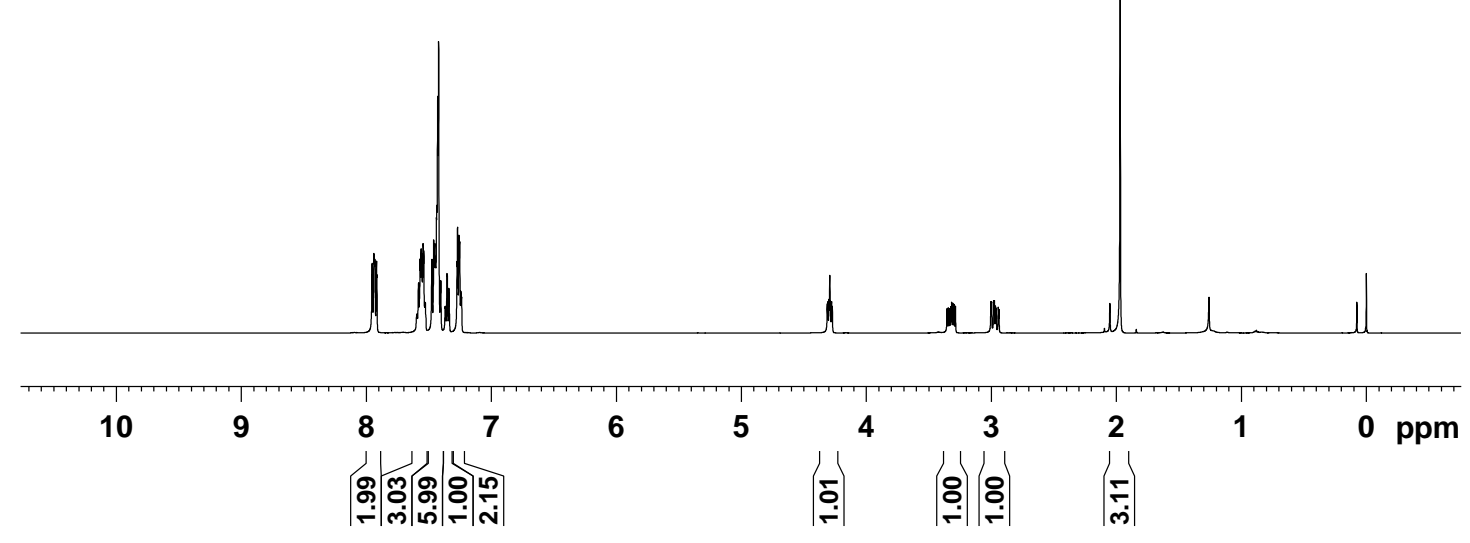


${ }^{13} \mathrm{C}$ NMR $\left(\mathrm{CDCl}_{3}, 125 \mathrm{MHz}\right)$ of $\mathbf{3 h}:$
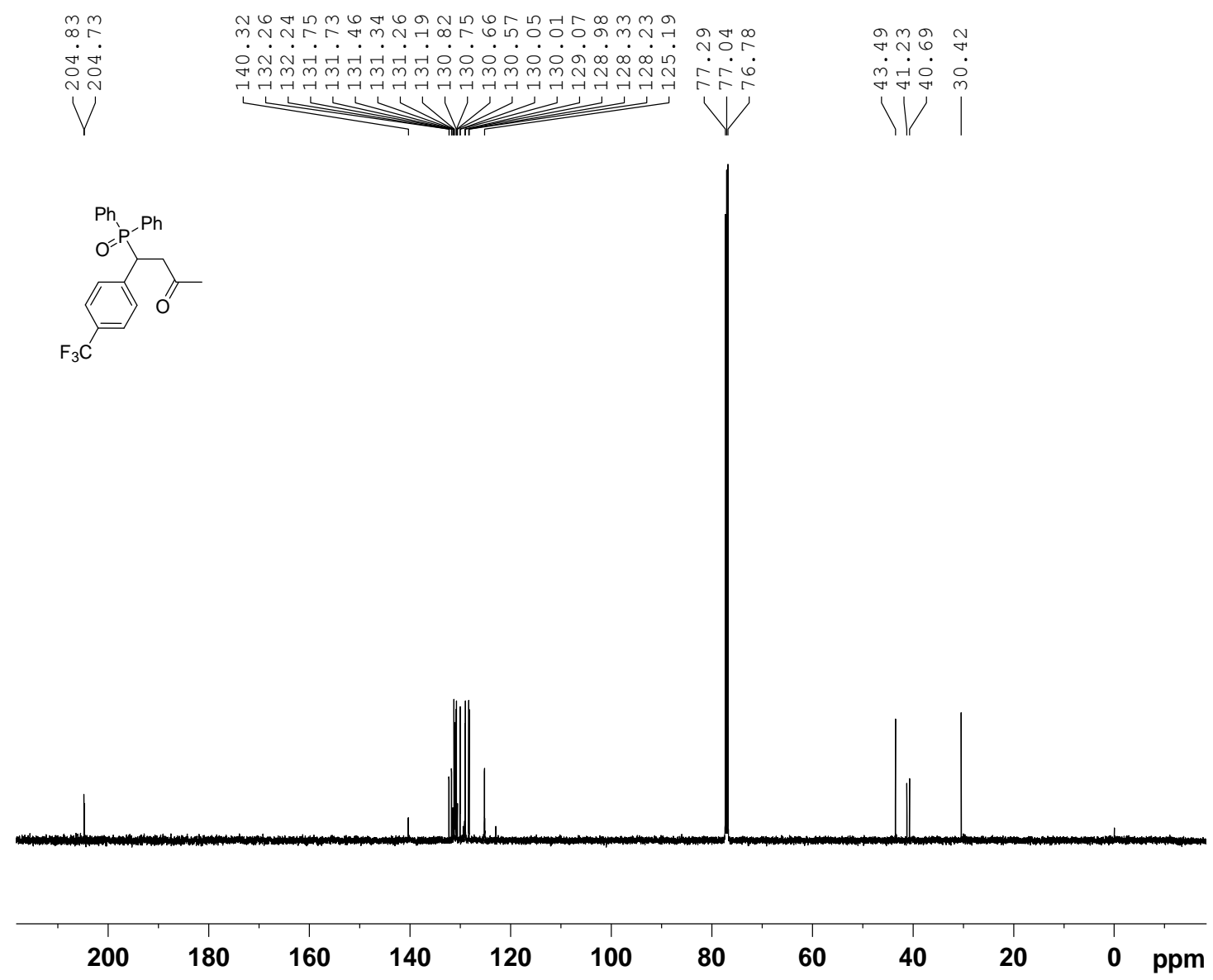

${ }^{31} \mathrm{P}$ NMR $\left(\mathrm{CDCl}_{3}, 202 \mathrm{MHz}\right)$ of $\mathbf{3 h}$ :
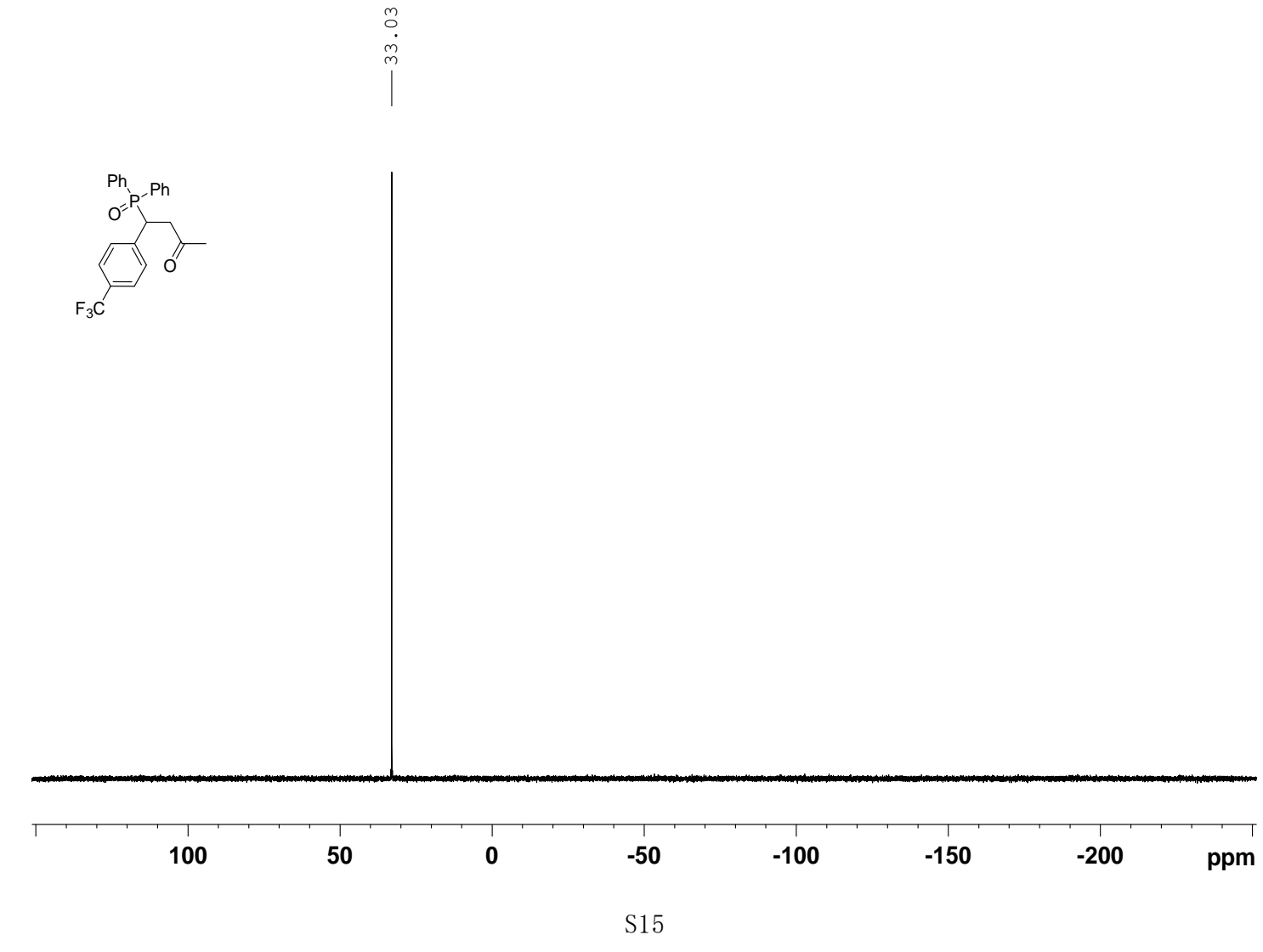
${ }^{1} \mathrm{H}$ NMR $\left(\mathrm{CDCl}_{3}, 500 \mathrm{MHz}\right)$ of $\mathbf{3 i}$ :
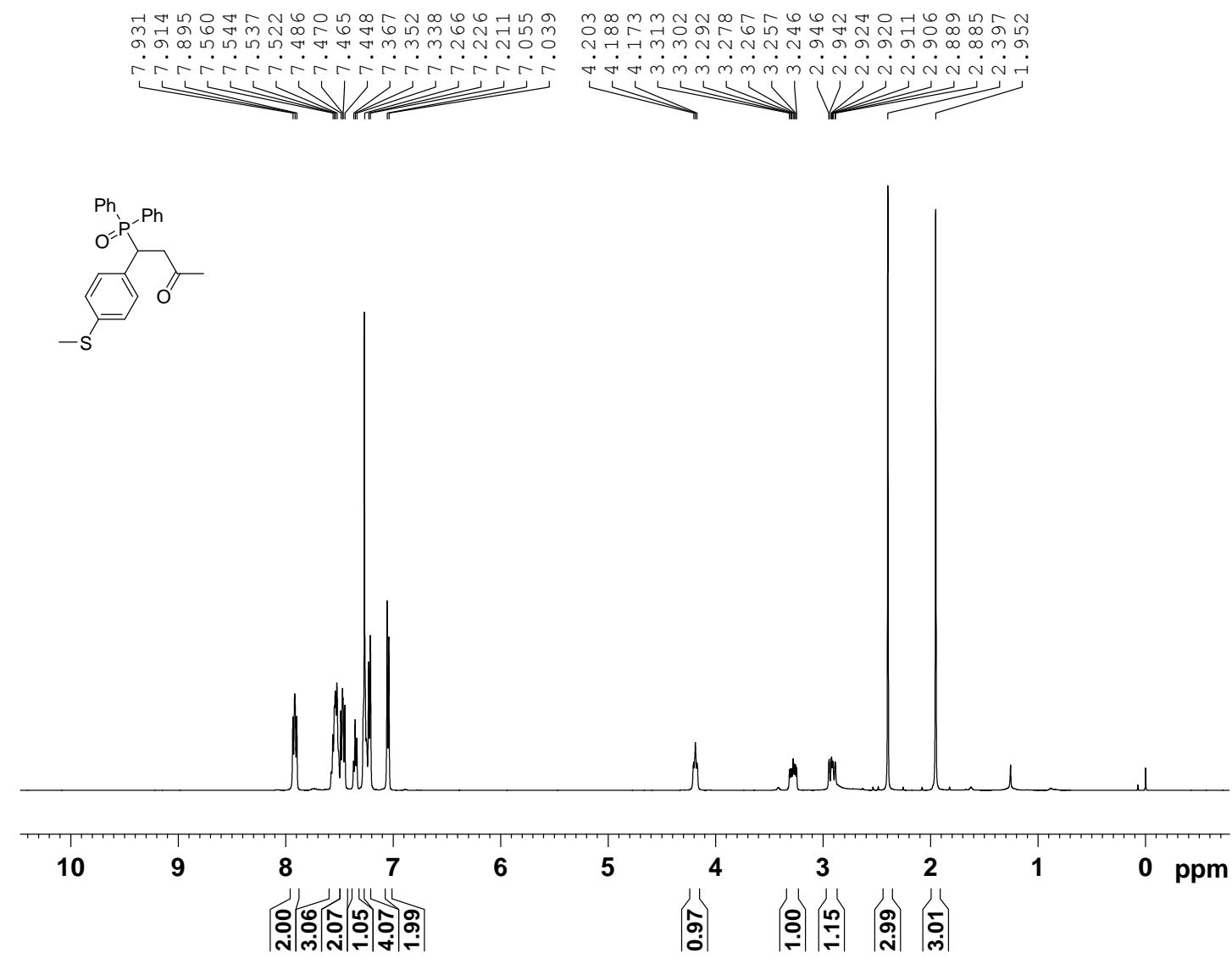

${ }^{13} \mathrm{C} \mathrm{NMR}\left(\mathrm{CDCl}_{3}, 125 \mathrm{MHz}\right)$ of $\mathbf{3 i}$ :

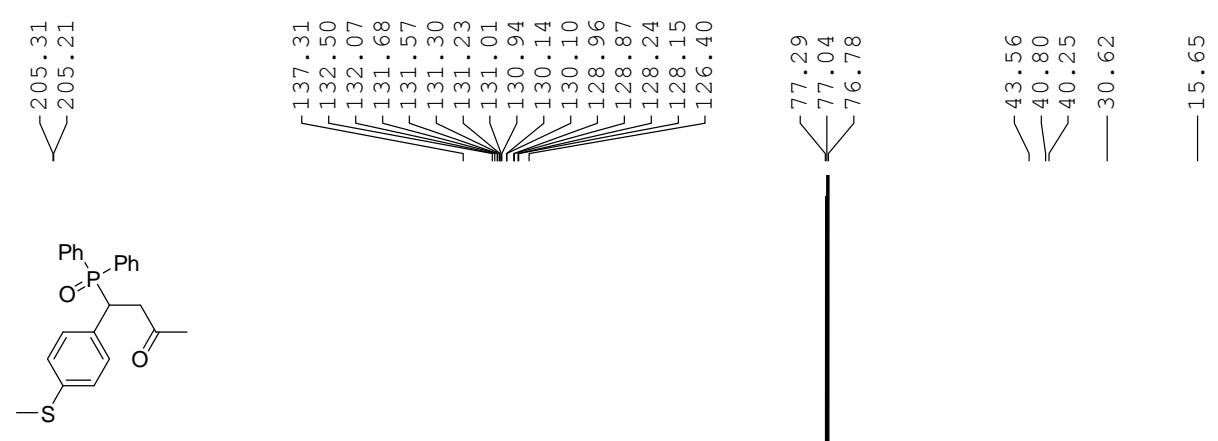

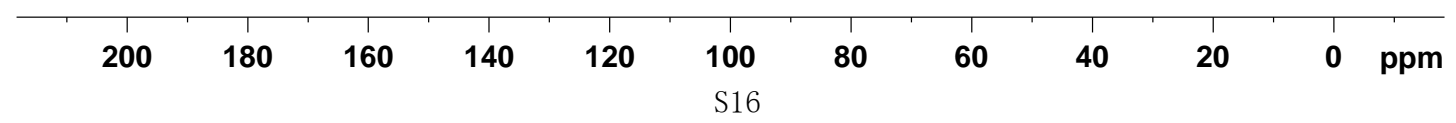


${ }^{31} \mathrm{P}$ NMR $\left(\mathrm{CDCl}_{3}, 202 \mathrm{MHz}\right)$ of $\mathbf{3 i}$ :
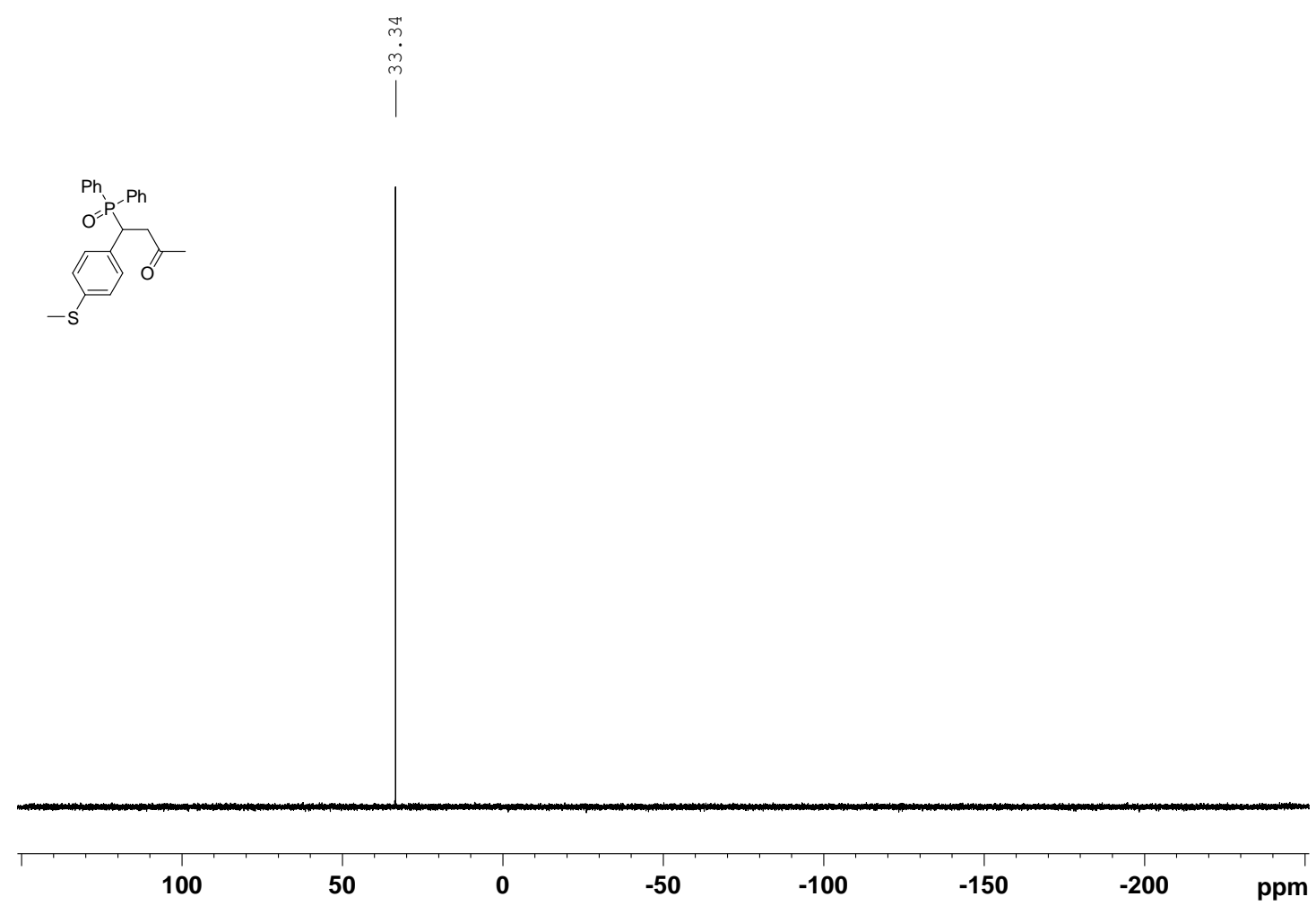

${ }^{1} \mathrm{H}$ NMR $\left(\mathrm{CDCl}_{3}, 500 \mathrm{MHz}\right)$ of $\mathbf{3 j}$ :

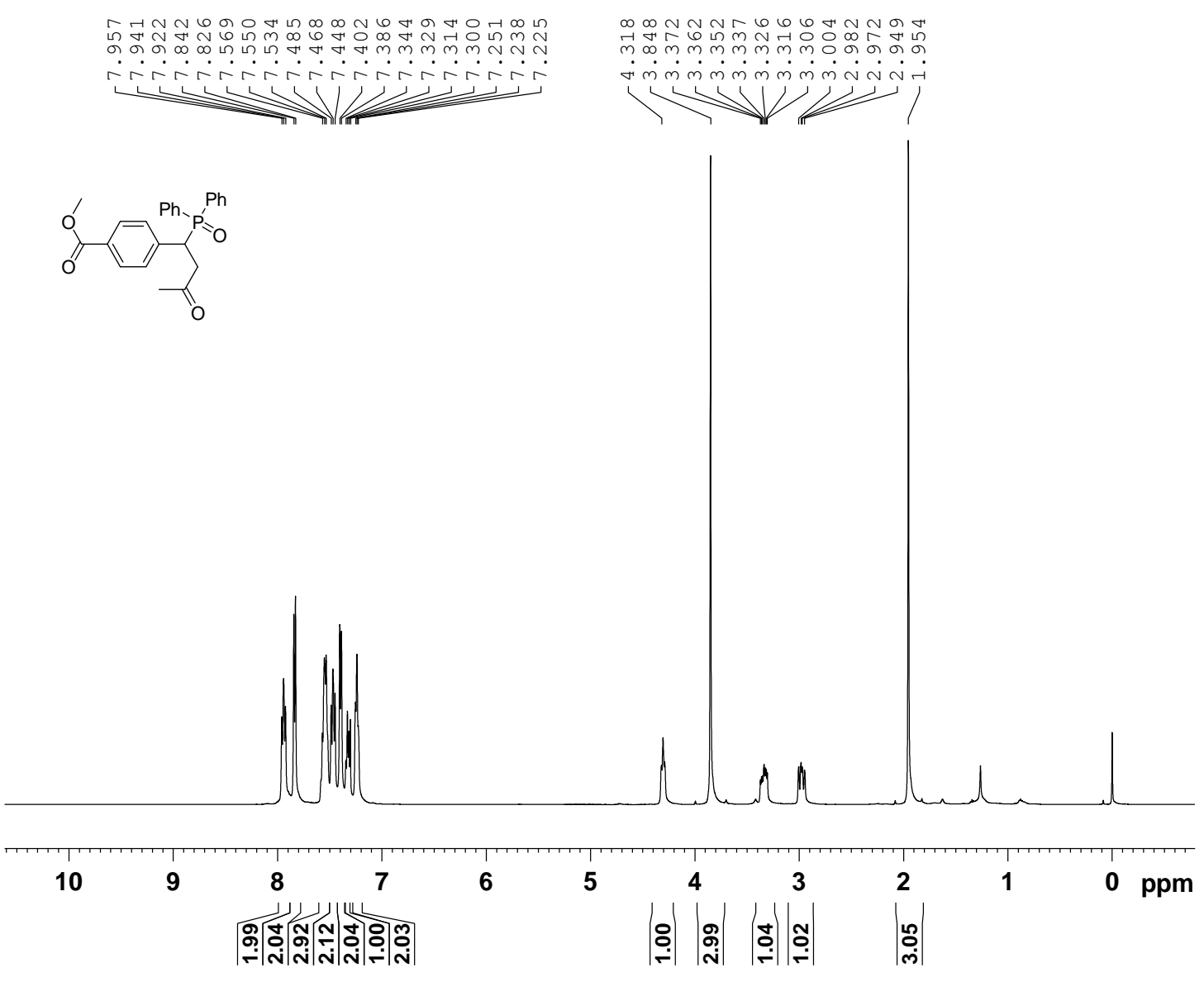


${ }^{13} \mathrm{C} \mathrm{NMR}\left(\mathrm{CDCl}_{3}, 125 \mathrm{MHz}\right)$ of $\mathbf{3 j}$ :

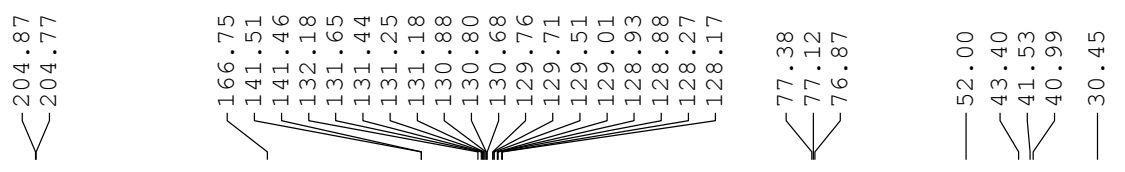

(1)

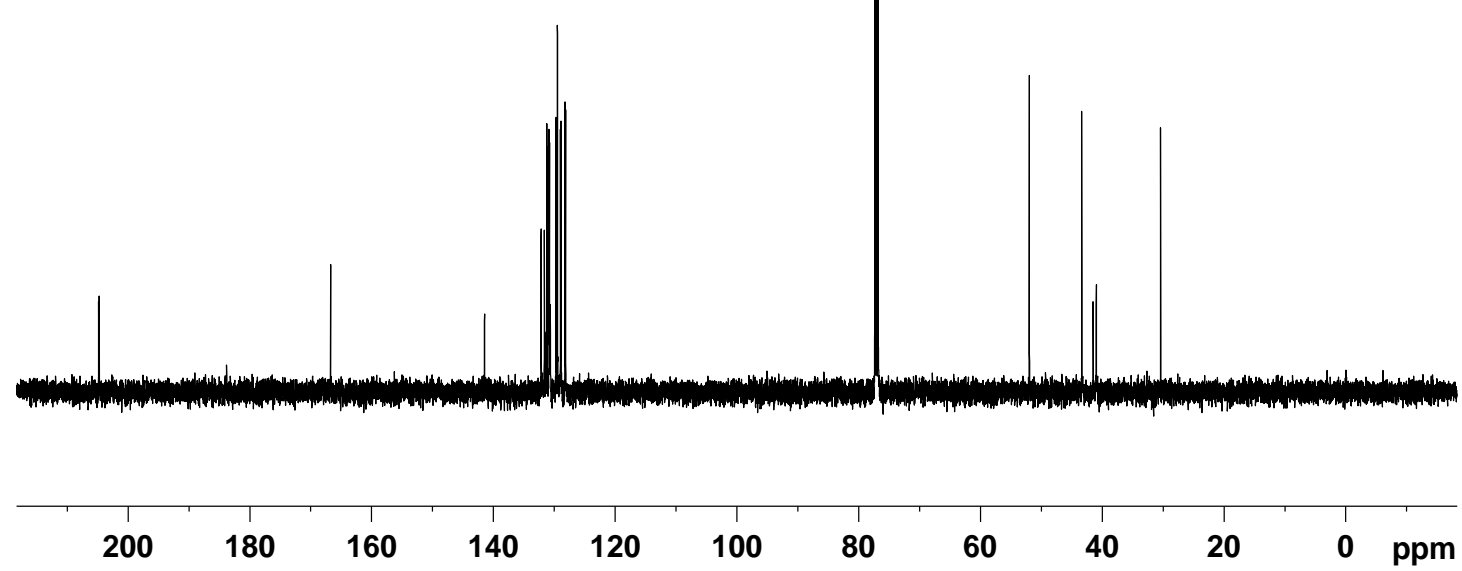

${ }^{31} \mathrm{P} \mathrm{NMR}\left(\mathrm{CDCl}_{3}, 202 \mathrm{MHz}\right)$ of $\mathbf{3 j}$ :
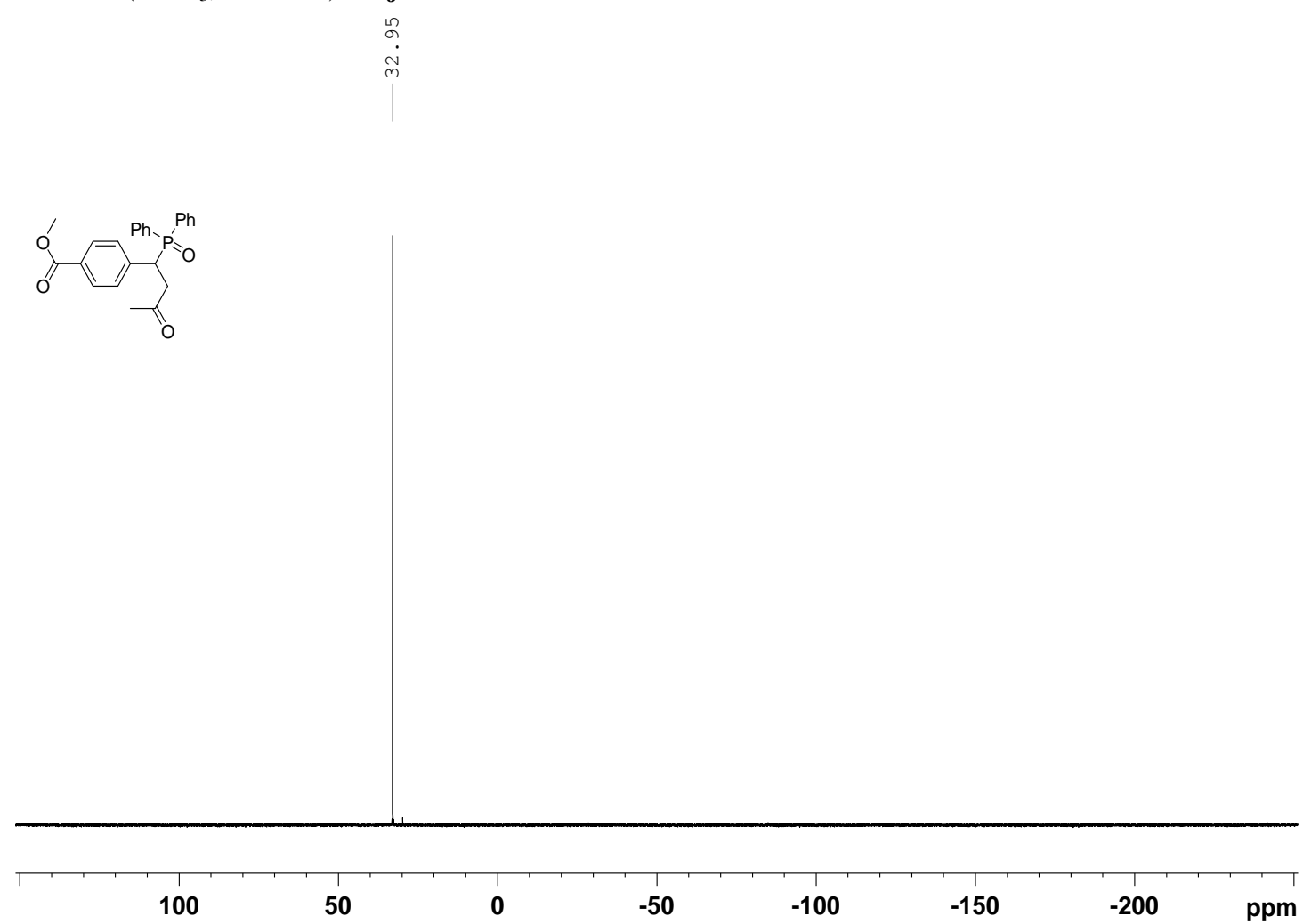
${ }^{1} \mathrm{H} \mathrm{NMR}\left(\mathrm{CDCl}_{3}, 500 \mathrm{MHz}\right)$ of $\mathbf{3 k}$ :

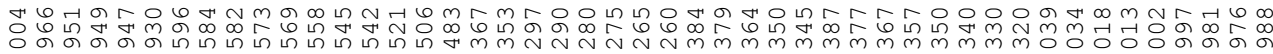

.

$\longrightarrow$

(O)

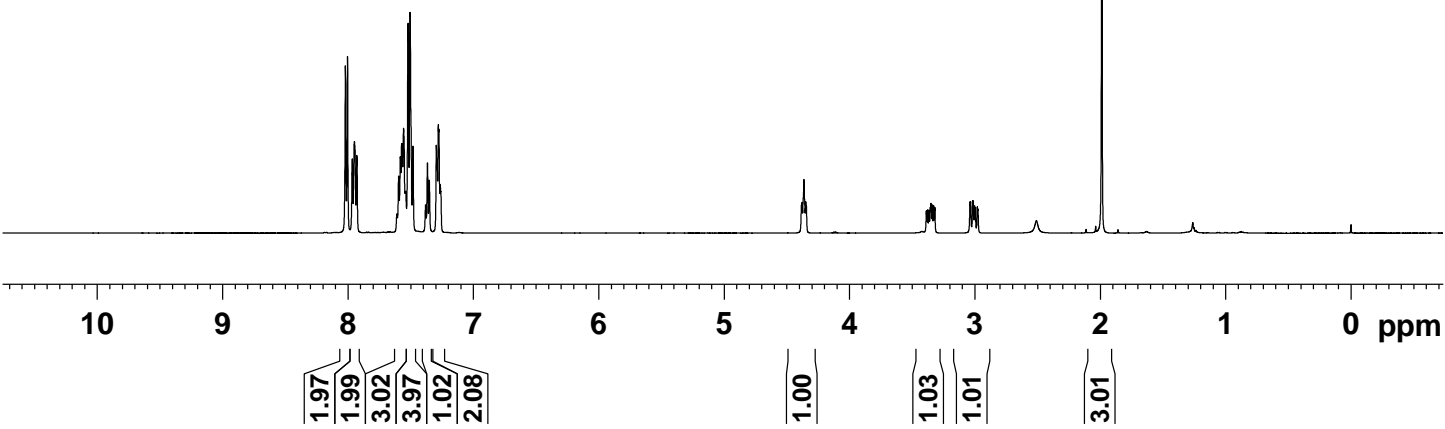

${ }^{13} \mathrm{C} \mathrm{NMR}\left(\mathrm{CDCl}_{3}, 125 \mathrm{MHz}\right)$ of $\mathbf{3 k}$ :
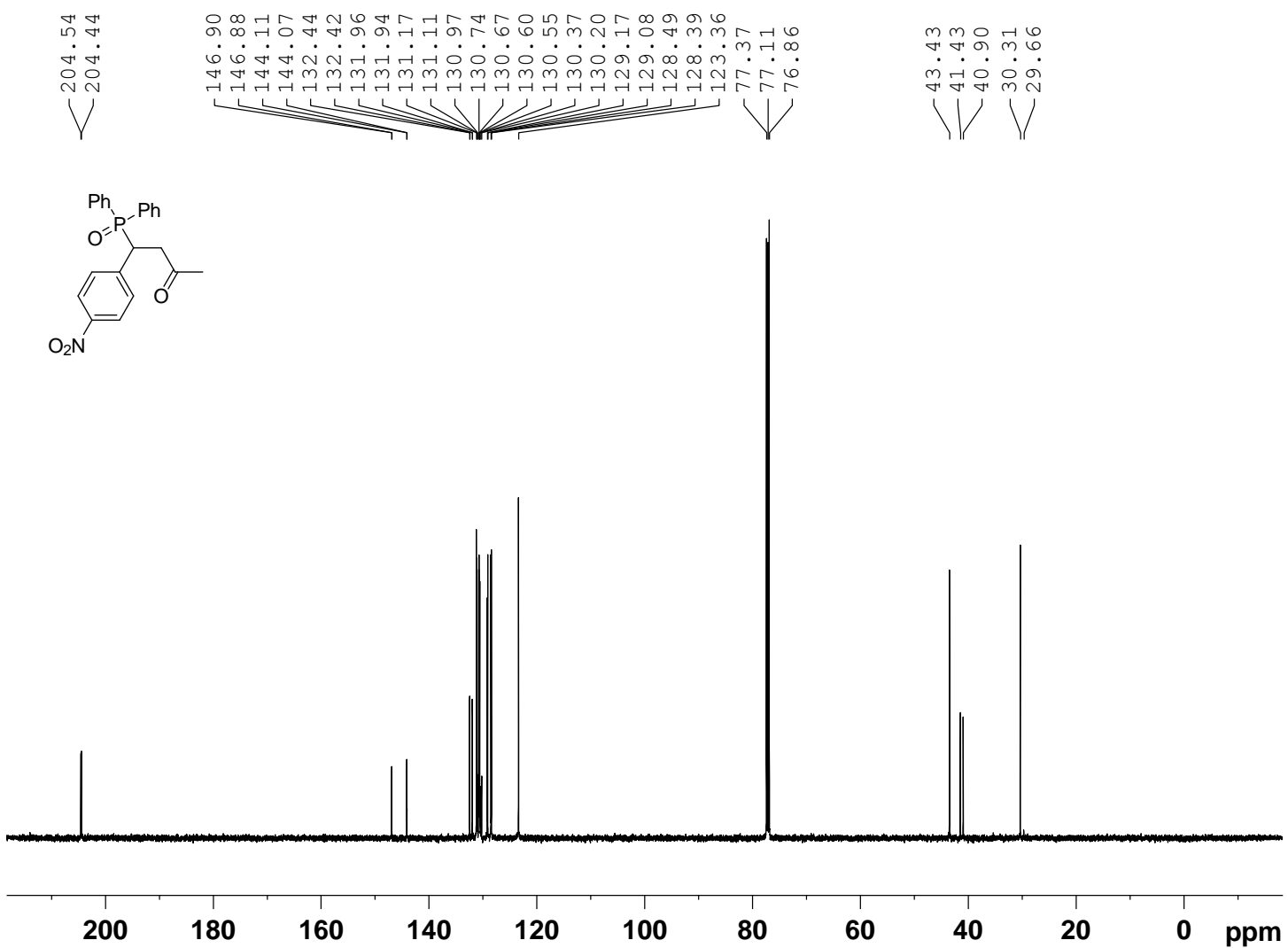
${ }^{31} \mathrm{P}$ NMR $\left(\mathrm{CDCl}_{3}, 202 \mathrm{MHz}\right)$ of $\mathbf{3 k}$ :
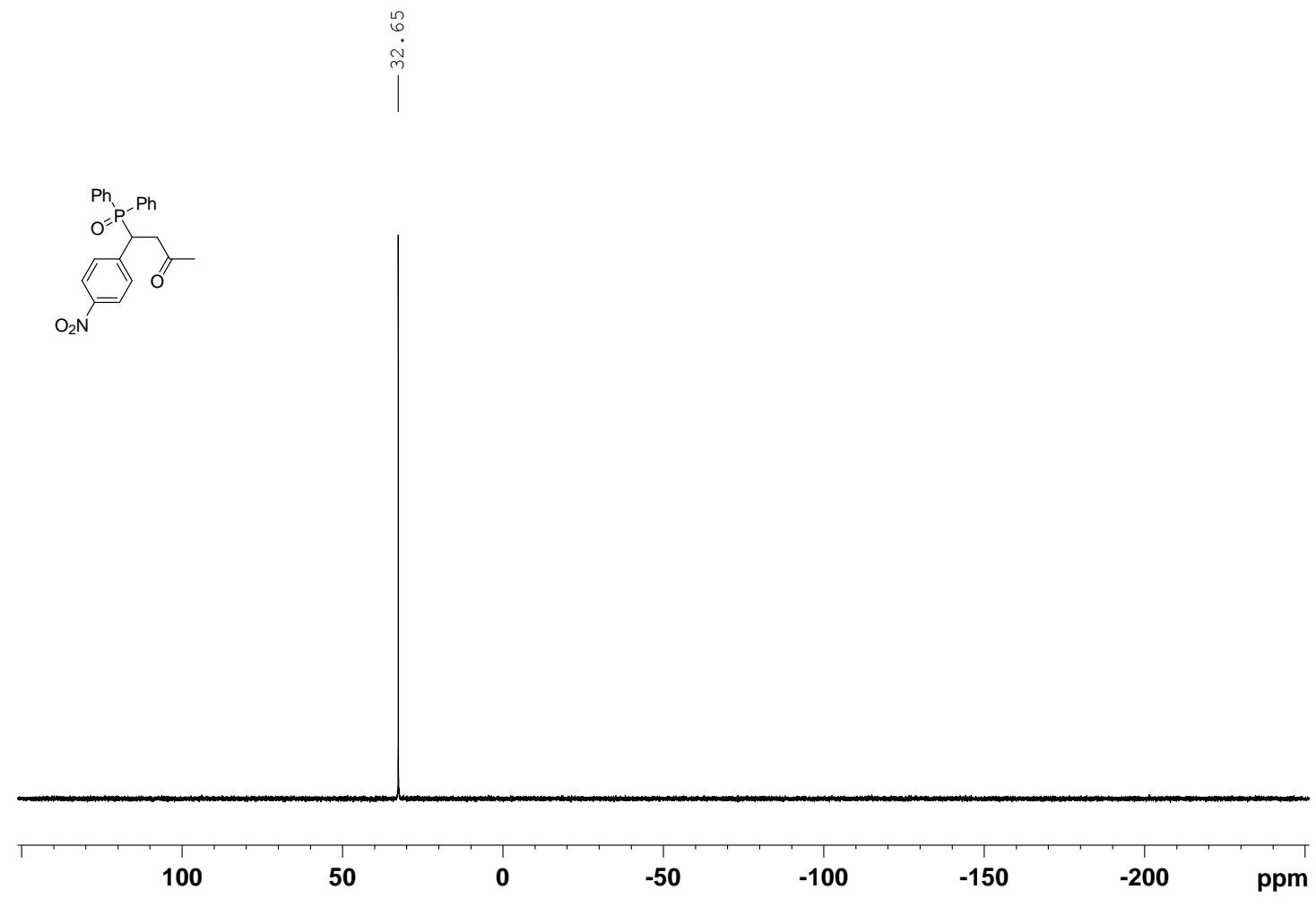

${ }^{1} \mathrm{H} \mathrm{NMR}\left(\mathrm{CDCl}_{3}, 500 \mathrm{MHz}\right)$ of 31:

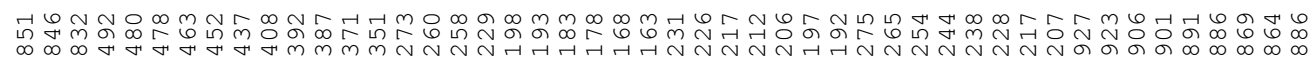

Nom

s.rum

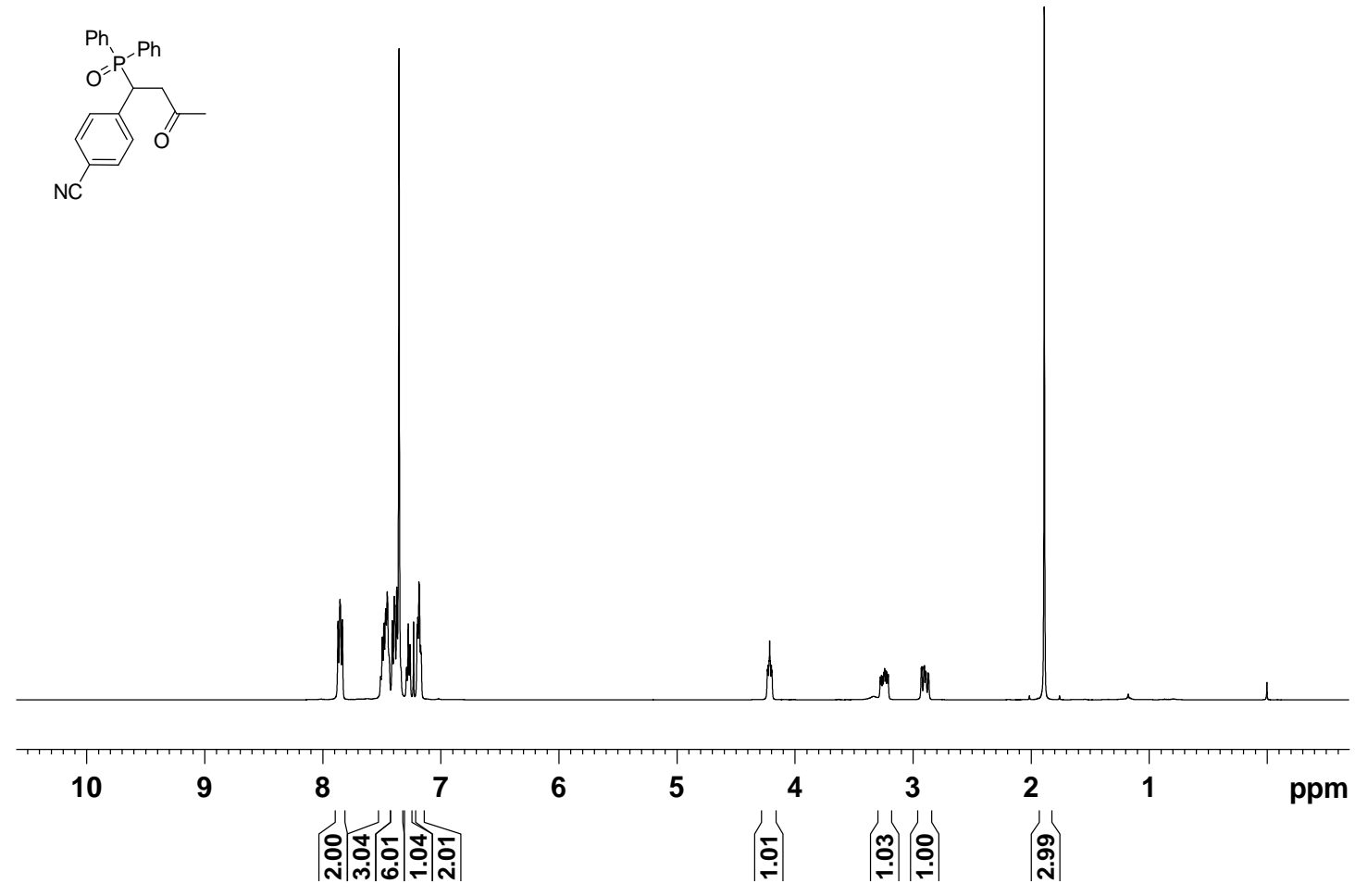


${ }^{13} \mathrm{C}$ NMR $\left(\mathrm{CDCl}_{3}, 125 \mathrm{MHz}\right)$ of 31:
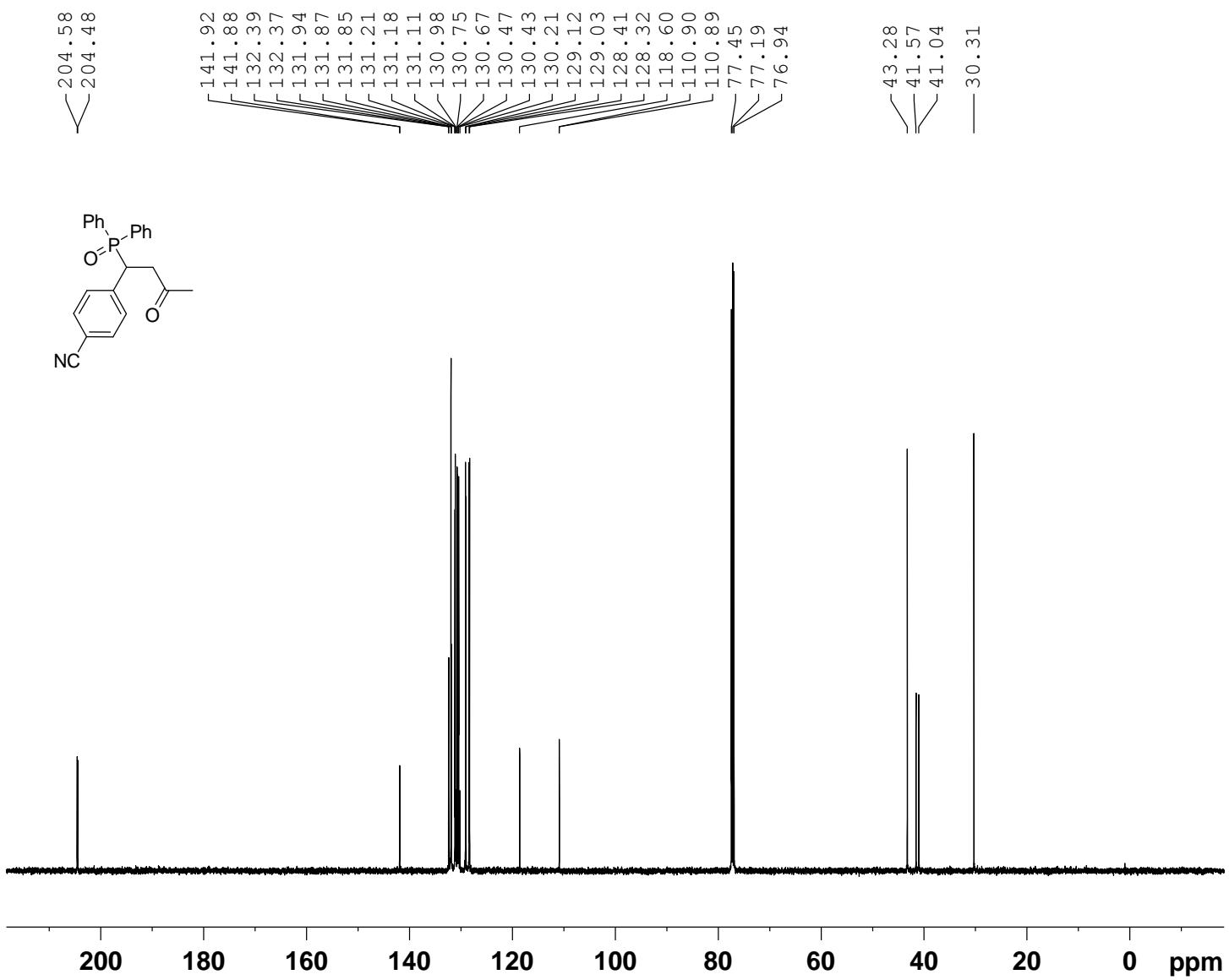

${ }^{31} \mathrm{P}$ NMR $\left(\mathrm{CDCl}_{3}, 202 \mathrm{MHz}\right)$ of 31:<smiles>CC(=O)CC(c1ccc(C#N)cc1)P(c1ccccc1)c1ccccc1</smiles>

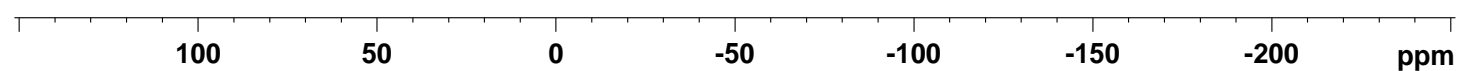


${ }^{1} \mathrm{H}$ NMR $\left(\mathrm{CDCl}_{3}, 500 \mathrm{MHz}\right)$ of $\mathbf{3 m}$ :

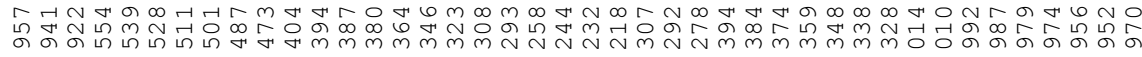

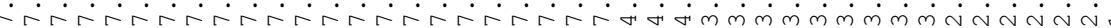

w1
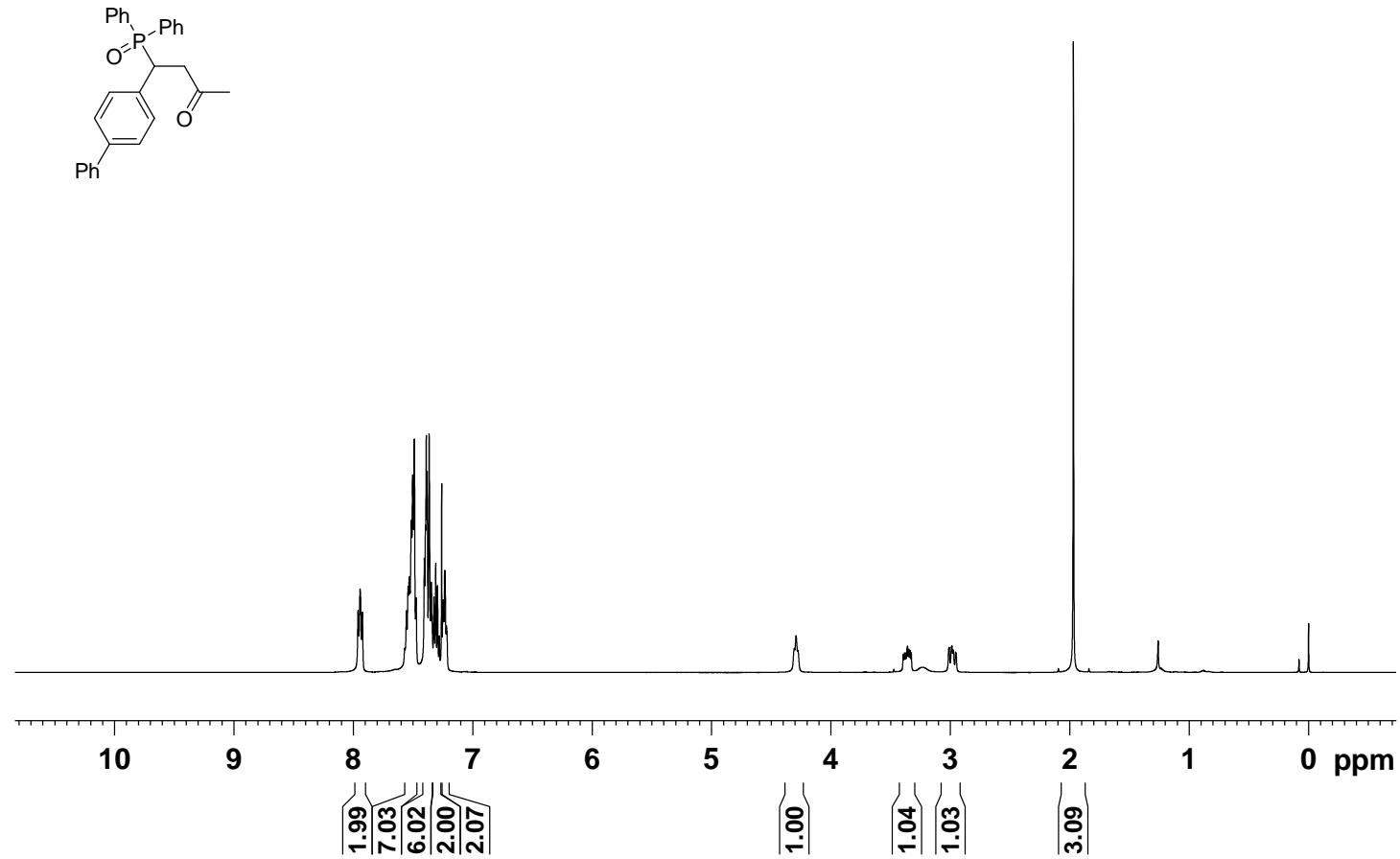

${ }^{13} \mathrm{C} \mathrm{NMR}\left(\mathrm{CDCl}_{3}, 125 \mathrm{MHz}\right)$ of $\mathbf{3 m}$ :
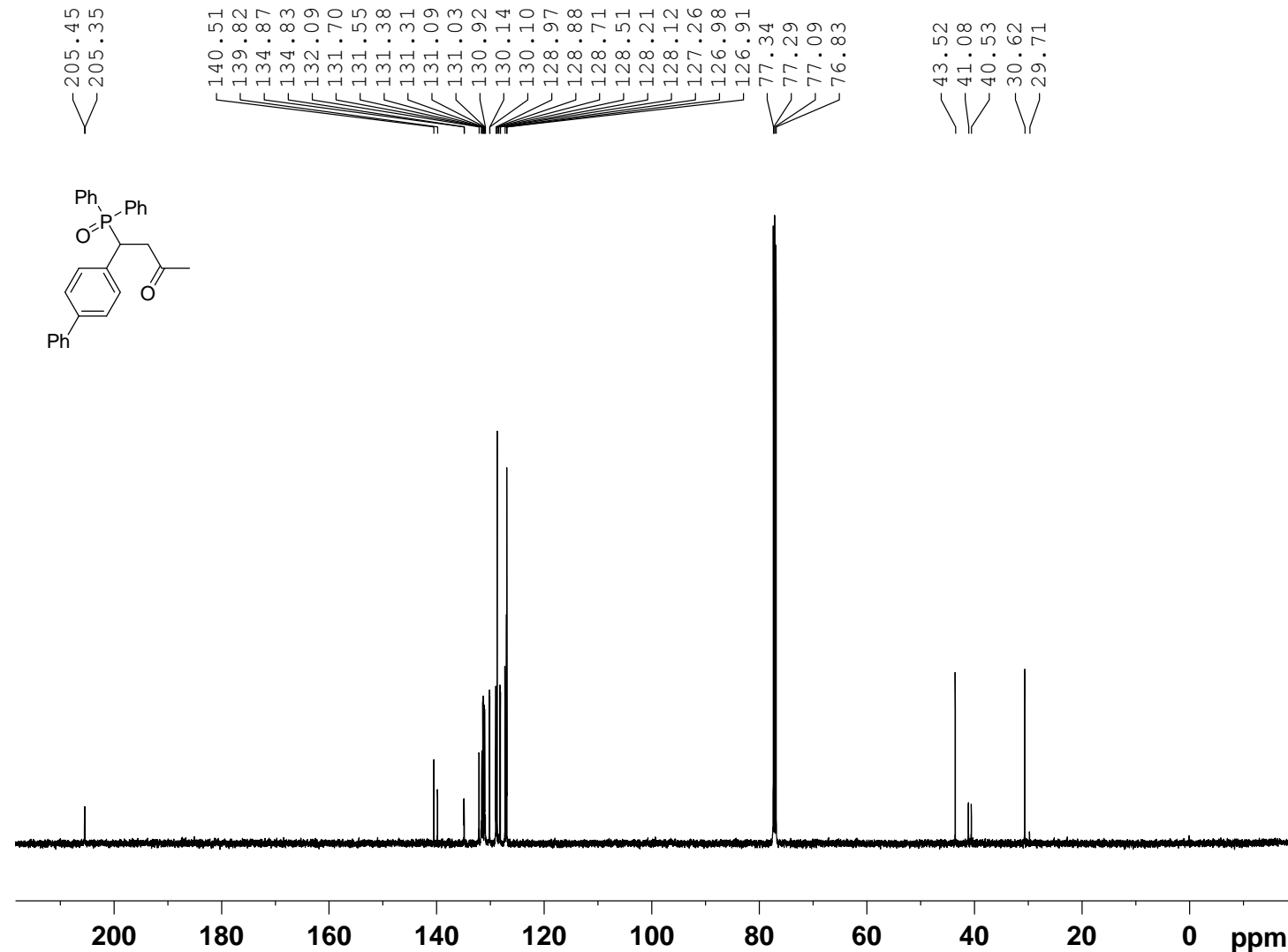
${ }^{31} \mathrm{P}$ NMR $\left(\mathrm{CDCl}_{3}, 202 \mathrm{MHz}\right)$ of $\mathbf{3 m}$ :
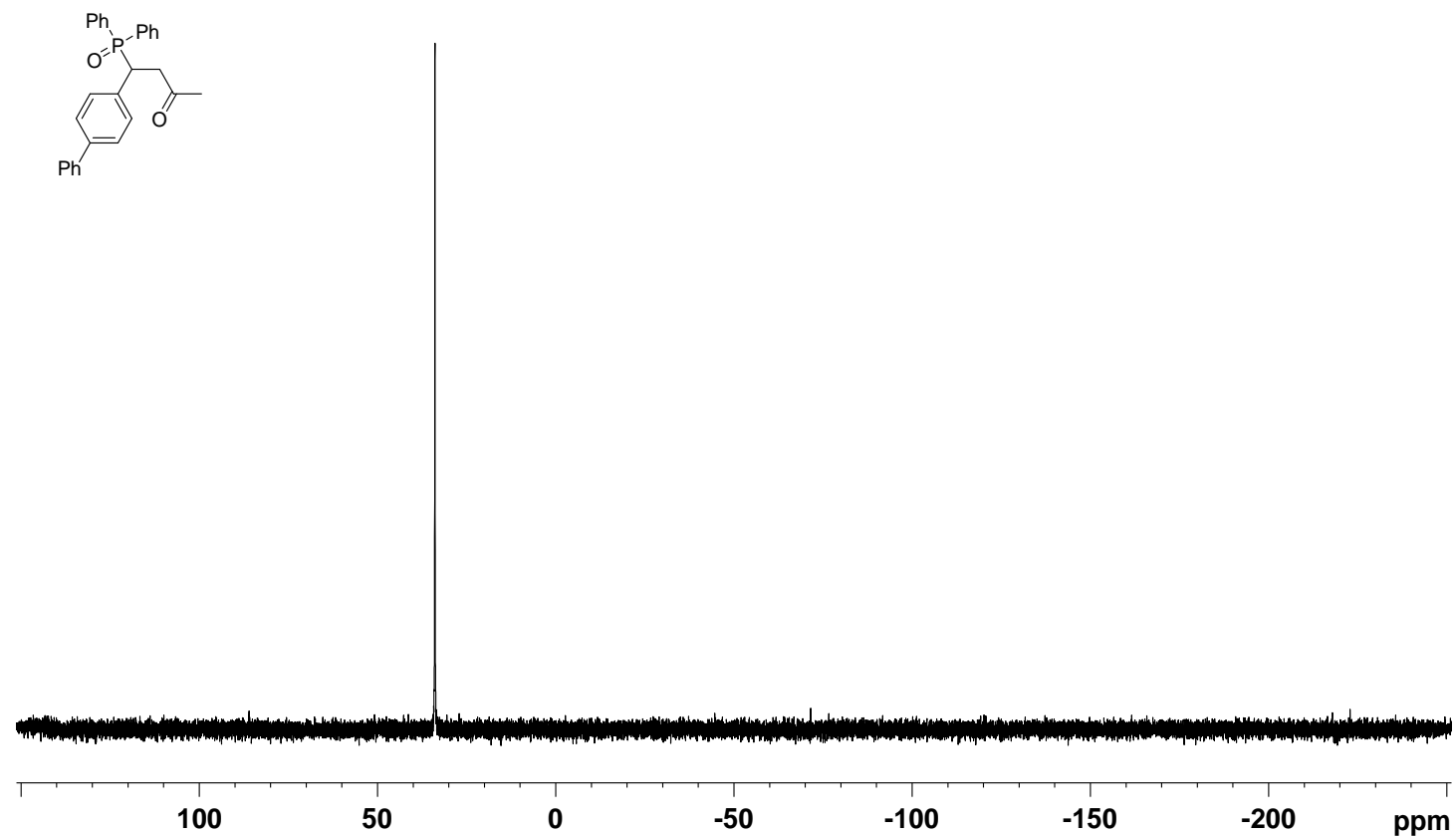

${ }^{1} \mathrm{H} \mathrm{NMR}\left(\mathrm{CDCl}_{3}, 500 \mathrm{MHz}\right)$ of $\mathbf{3 n}$ :

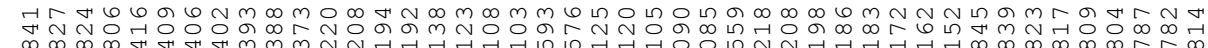
1<smiles>COc1ccc(C(CC(C)=O)P(=O)(c2ccccc2)c2ccccc2)cc1</smiles>

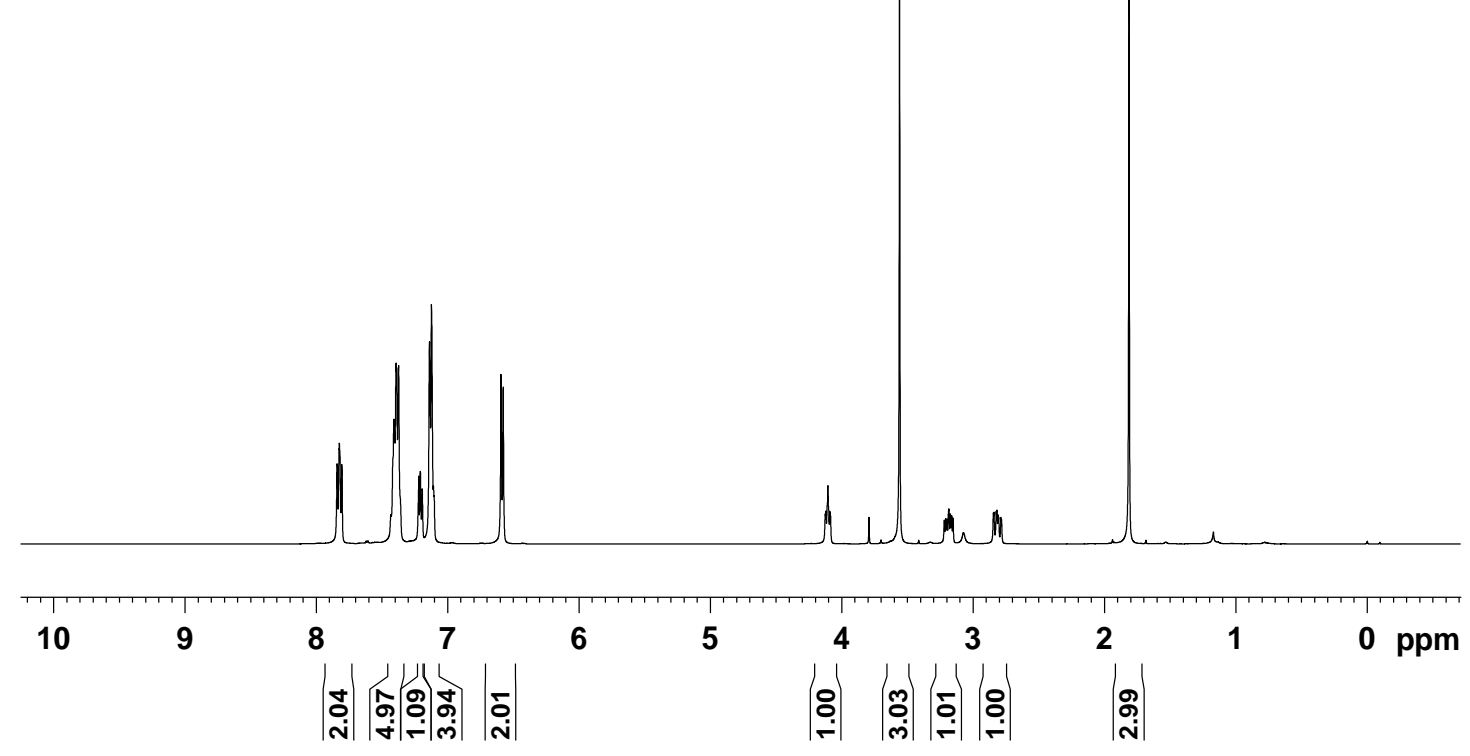


${ }^{13} \mathrm{C}$ NMR $\left(\mathrm{CDCl}_{3}, 125 \mathrm{MHz}\right)$ of $\mathbf{3 n}$ :
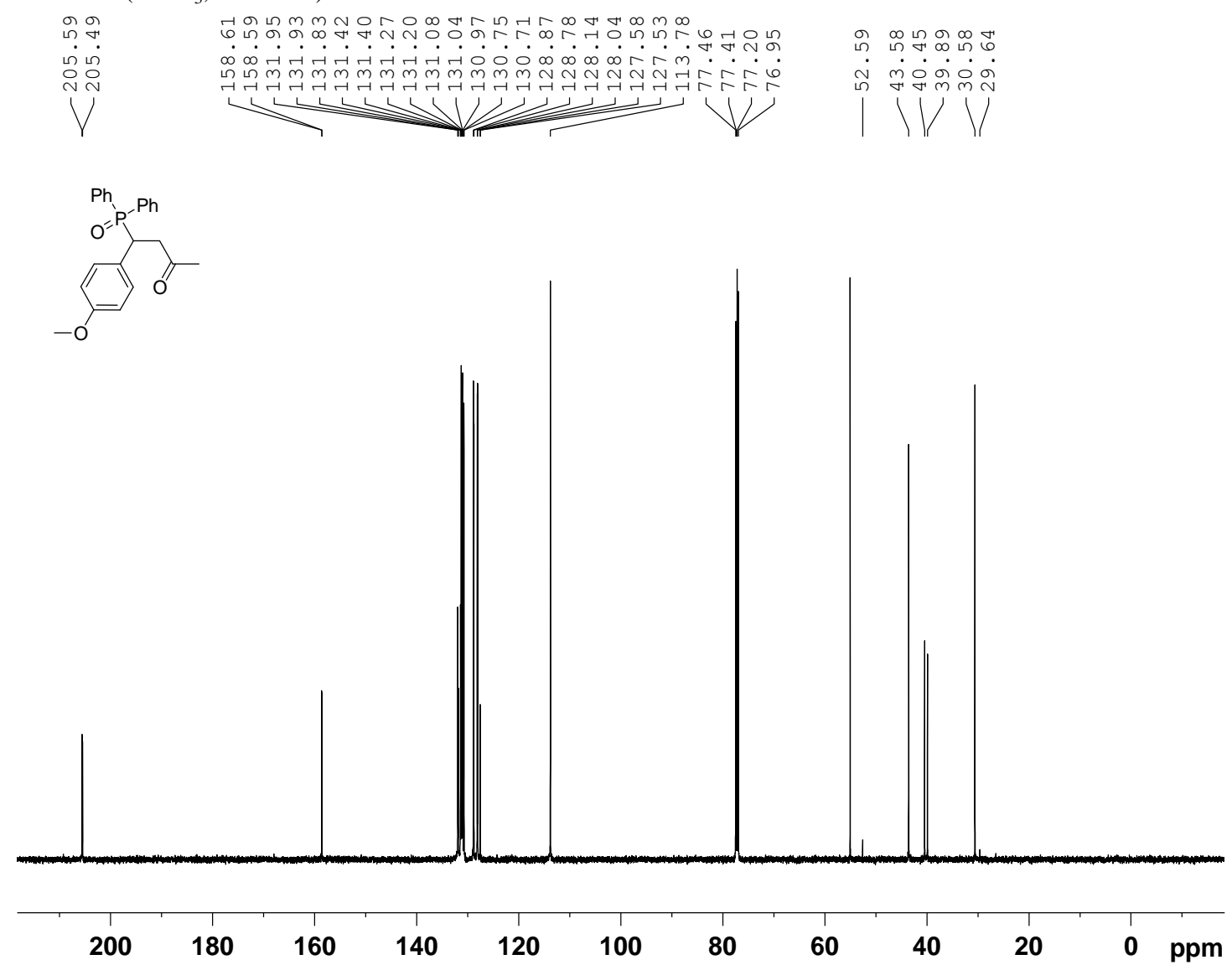

${ }^{31} \mathrm{P}$ NMR $\left(\mathrm{CDCl}_{3}, 202 \mathrm{MHz}\right)$ of $\mathbf{3 n}$ :
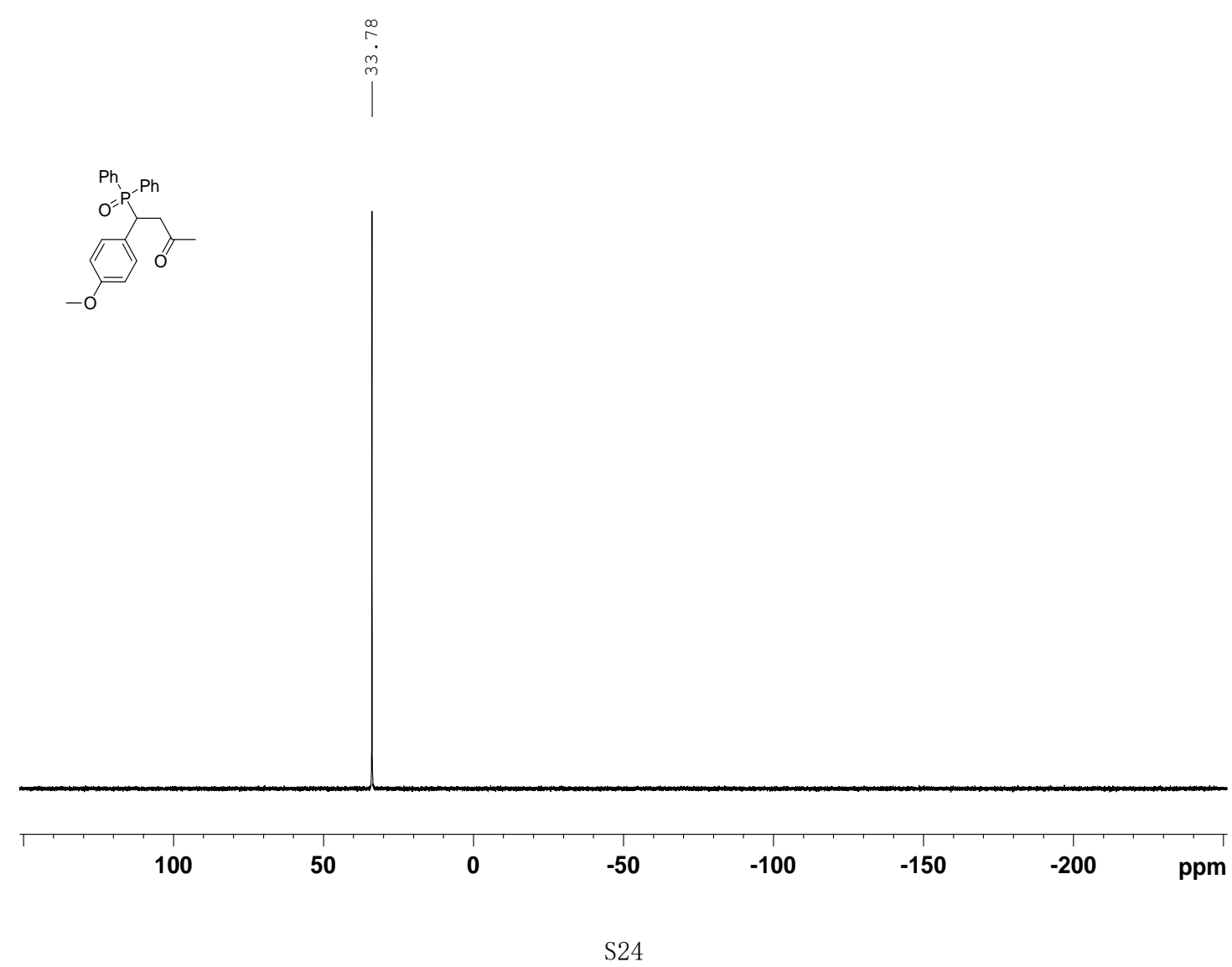
${ }^{1} \mathrm{H} \mathrm{NMR}\left(\mathrm{CDCl}_{3}, 500 \mathrm{MHz}\right)$ of $\mathbf{3 o}$ :

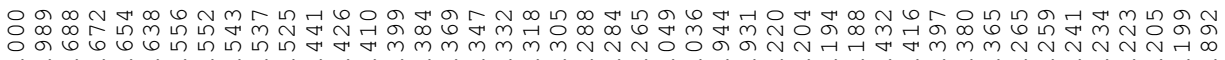

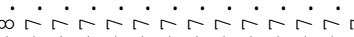

$m m m m m m m m$
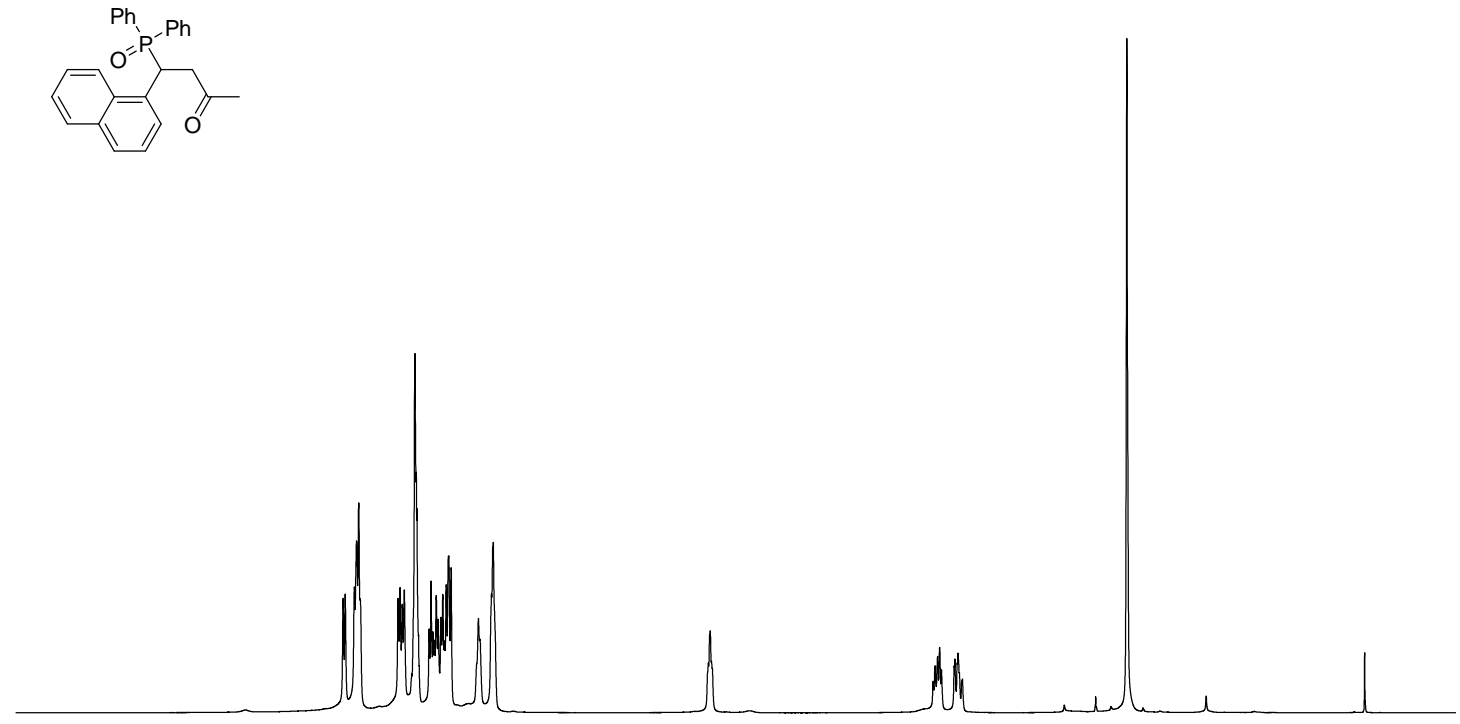

10

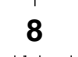

7

8 ใิ

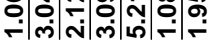

6

|

3

2

0 ppm

${ }^{13} \mathrm{C} \mathrm{NMR}\left(\mathrm{CDCl}_{3}, 125 \mathrm{MHz}\right)$ of $\mathbf{3 o}$ :
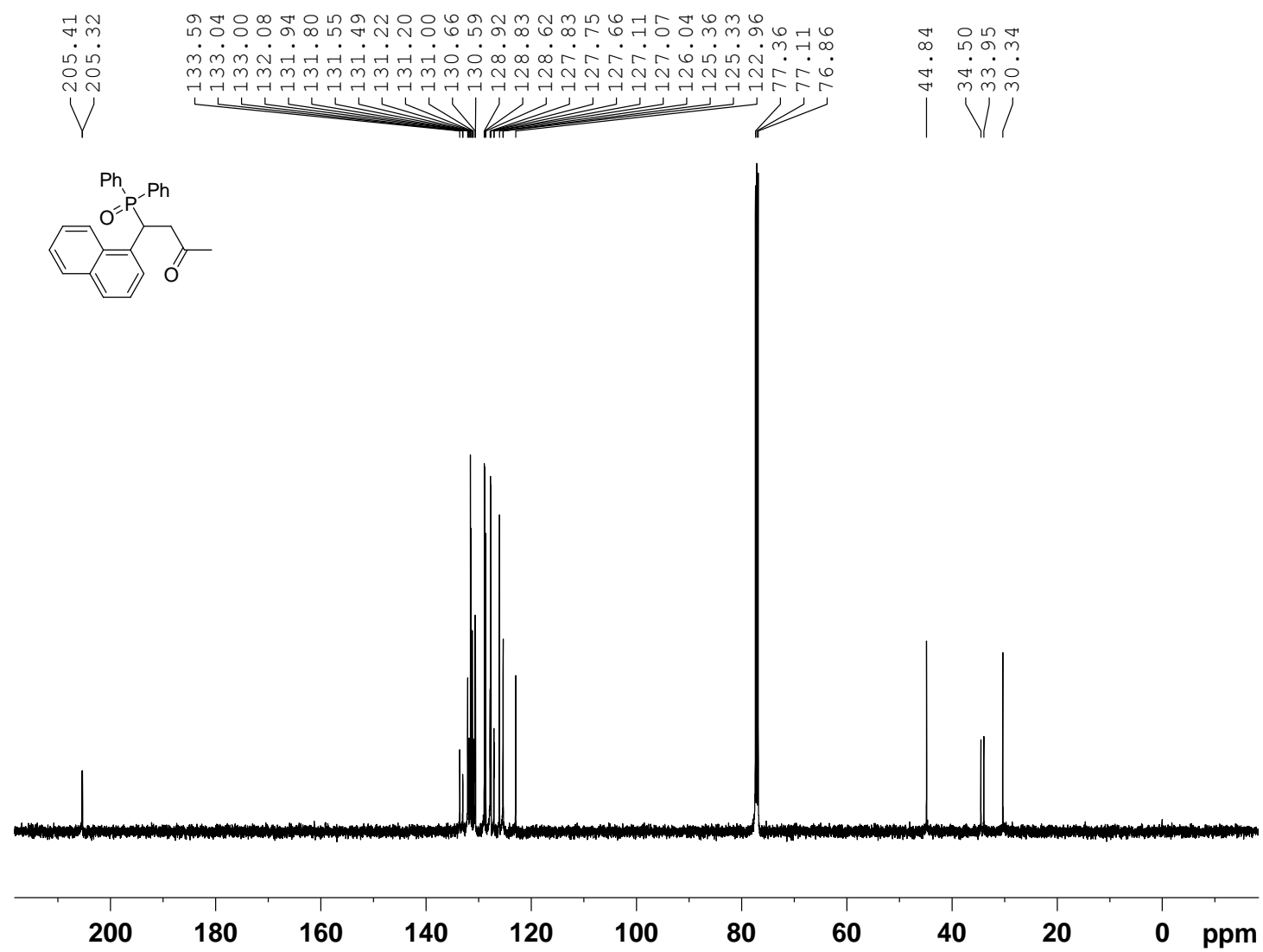
${ }^{31} \mathrm{P} \mathrm{NMR}\left(\mathrm{CDCl}_{3}, 202 \mathrm{MHz}\right)$ of $\mathbf{3 o}$ :

$$
\left.\right|^{\infty}
$$

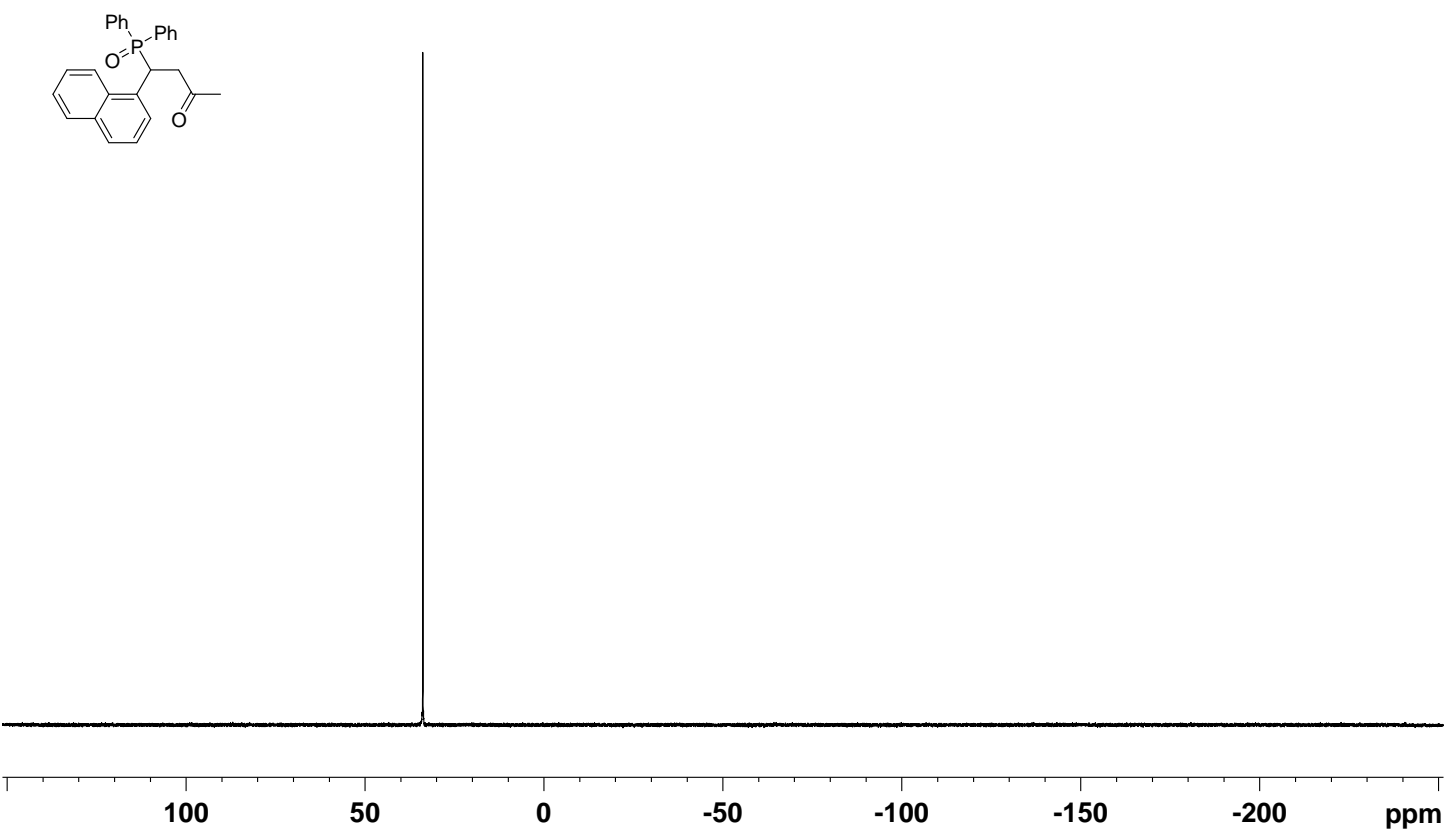

${ }^{1} \mathrm{H} \mathrm{NMR}\left(\mathrm{CDCl}_{3}, 500 \mathrm{MHz}\right)$ of $\mathbf{3 p}$ :

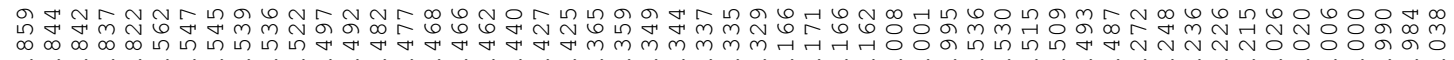

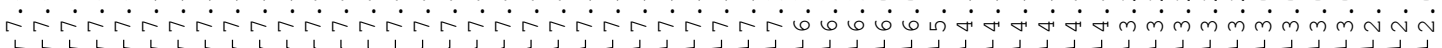<smiles>CC(=O)CC(c1ccco1)P(=O)(c1ccccc1)c1ccccc1</smiles>

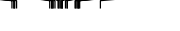

$\longrightarrow$

(1)

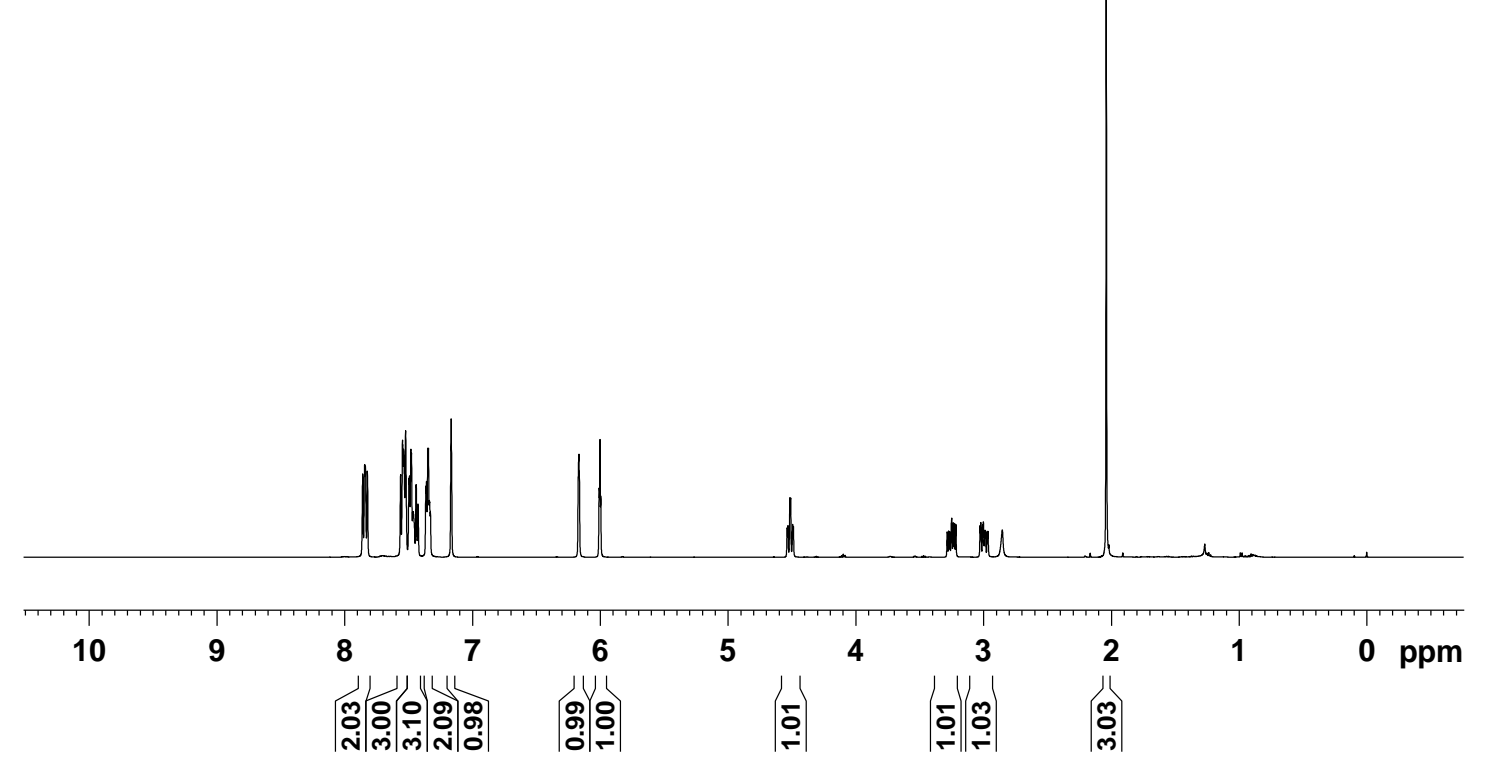


${ }^{13} \mathrm{C}$ NMR $\left(\mathrm{CDCl}_{3}, 125 \mathrm{MHz}\right)$ of $\mathbf{3 p}$ :

$\begin{array}{llll}\infty & \\ \infty & 0\end{array}$

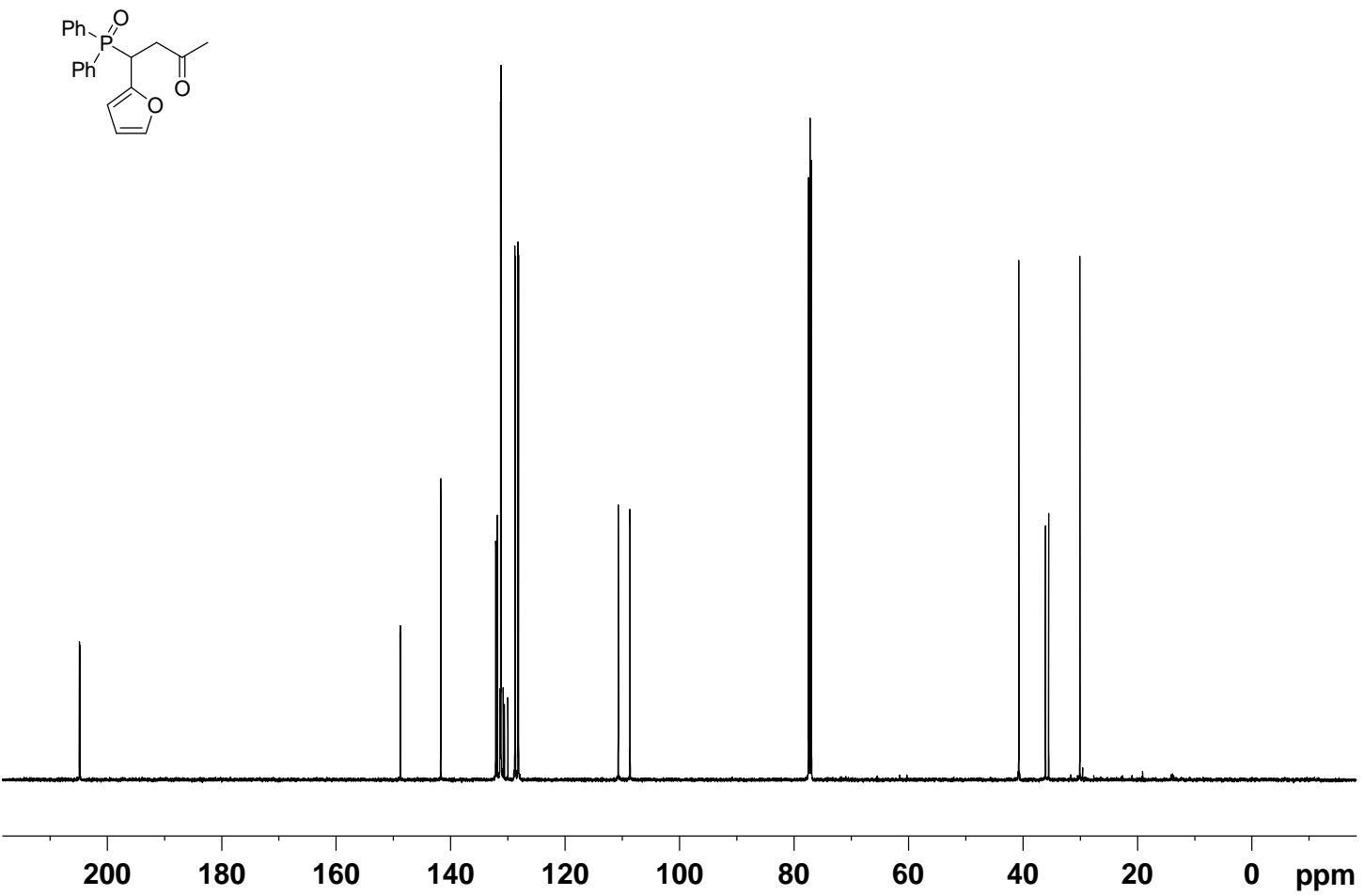

${ }^{31} \mathrm{P}$ NMR $\left(\mathrm{CDCl}_{3}, 202 \mathrm{MHz}\right)$ of $\mathbf{3 p}$ :

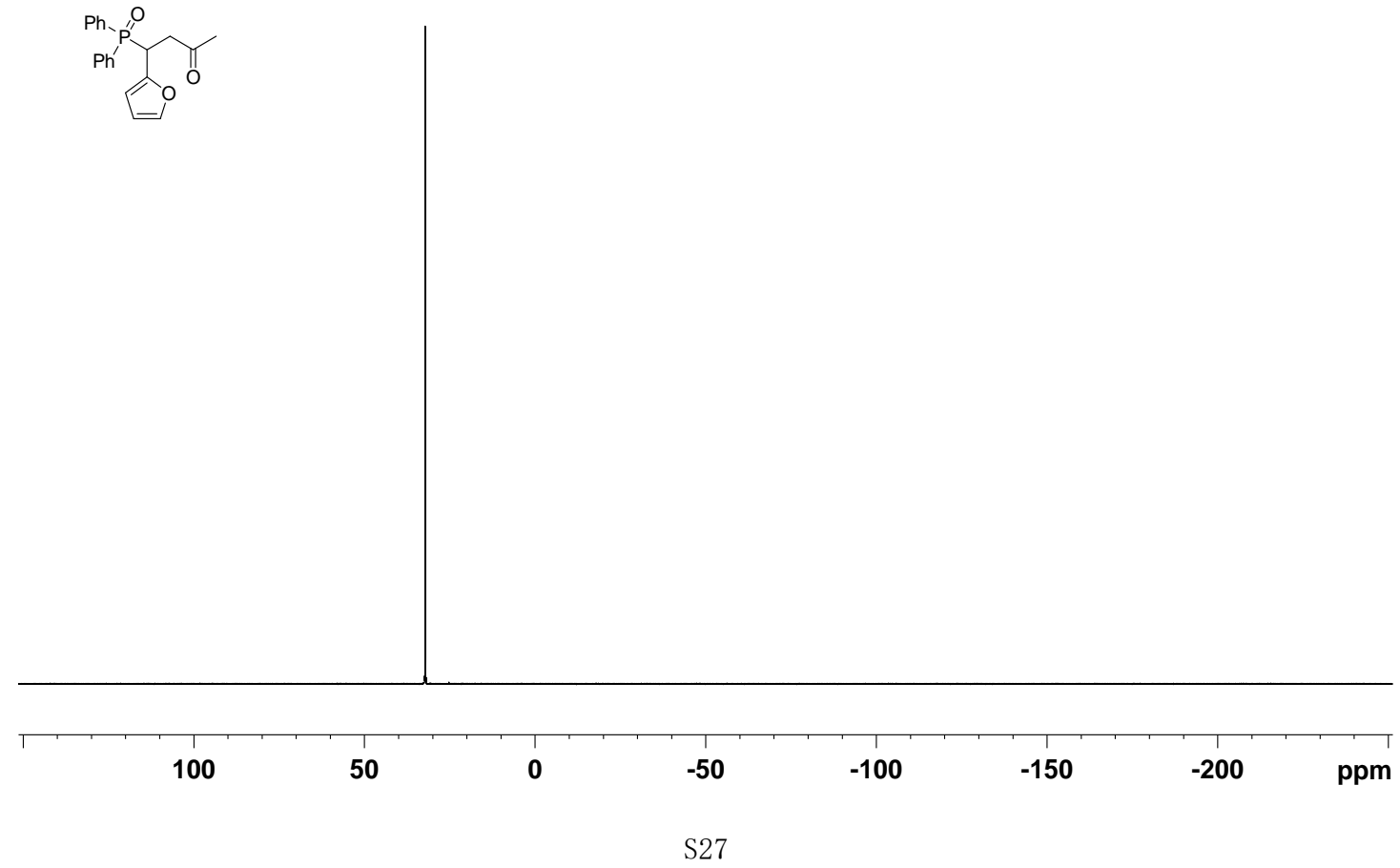


${ }^{1} \mathrm{H} \mathrm{NMR}\left(\mathrm{CDCl}_{3}, 500 \mathrm{MHz}\right)$ of $\mathbf{3 q}$ :

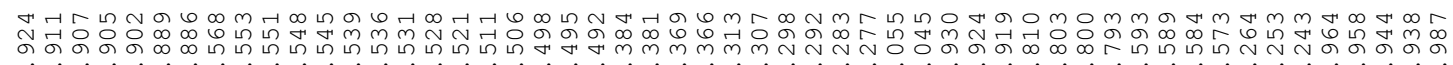

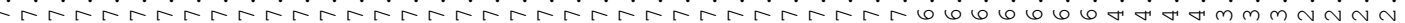

OS

$r$ 
${ }^{31} \mathrm{P} \mathrm{NMR}\left(\mathrm{CDCl}_{3}, 202 \mathrm{MHz}\right)$ of $\mathbf{3 q}$ :

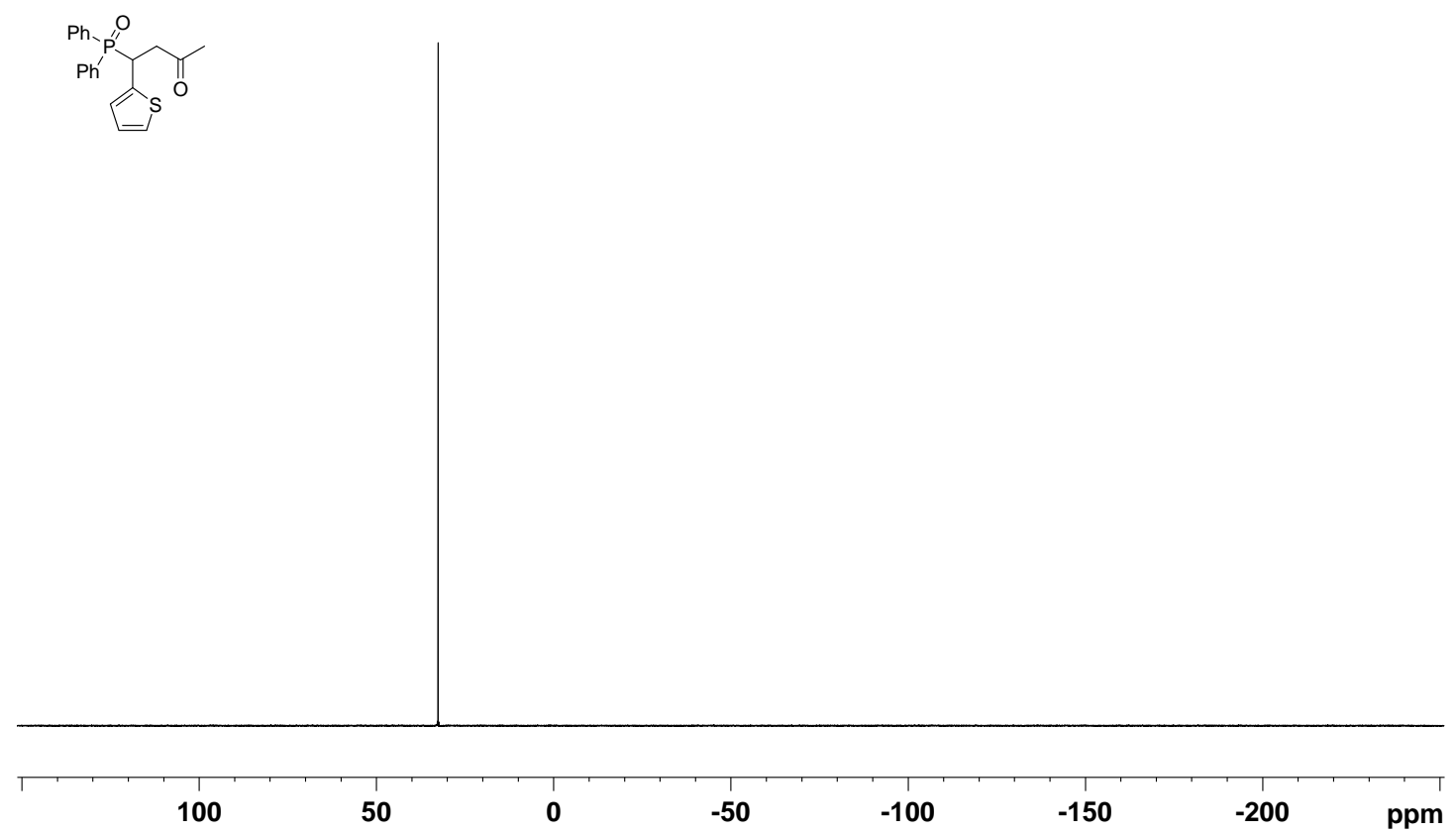

${ }^{1} \mathrm{H} \mathrm{NMR}\left(\mathrm{CDCl}_{3}, 500 \mathrm{MHz}\right)$ of $\mathbf{3 r}$ :

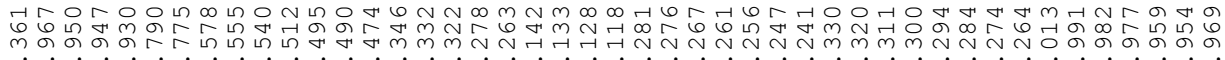

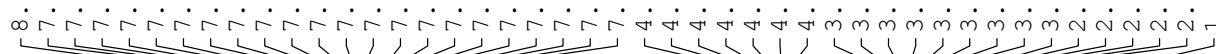

(n)

(1)

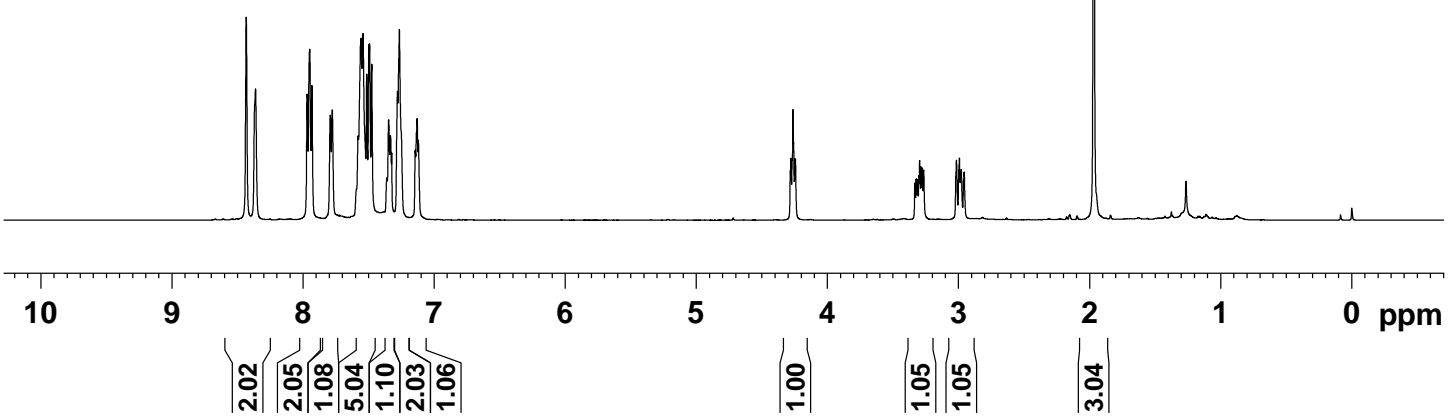


${ }^{13} \mathrm{C} \mathrm{NMR}\left(\mathrm{CDCl}_{3}, 125 \mathrm{MHz}\right)$ of $\mathbf{3 r}$ :

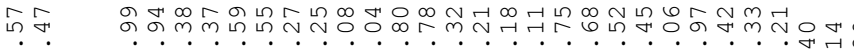

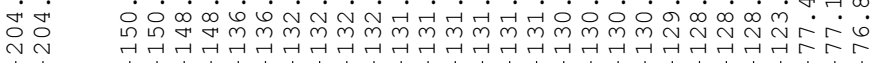

Y

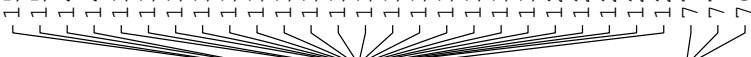

r

$m i \infty$

|V|<smiles>CC(=O)CC(c1ccccn1)P(=O)(c1ccccc1)c1ccccc1</smiles>

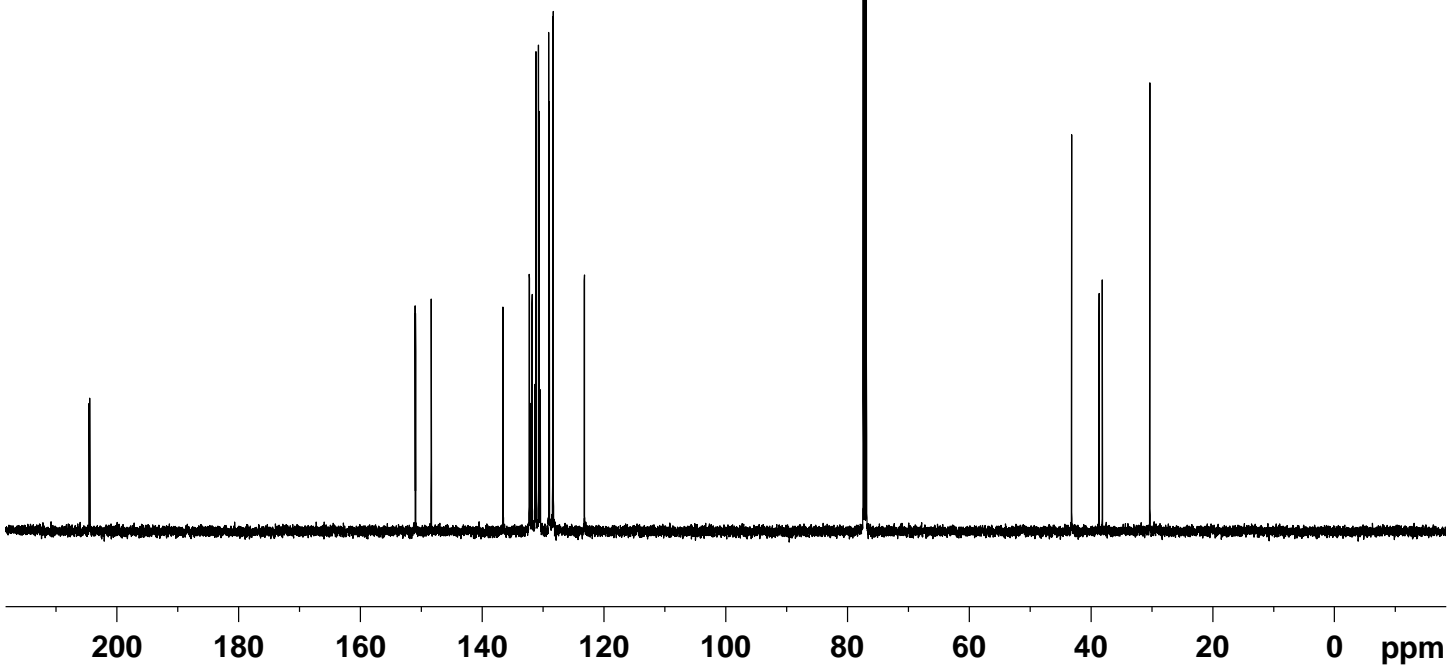

${ }^{31} \mathrm{P}$ NMR $\left(\mathrm{CDCl}_{3}, 202 \mathrm{MHz}\right)$ of $\mathbf{3 r}$
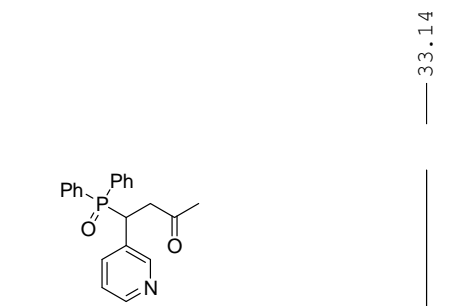
${ }^{1} \mathrm{H} \mathrm{NMR}\left(\mathrm{CDCl}_{3}, 500 \mathrm{MHz}\right)$ of $\mathbf{3 s}$ :

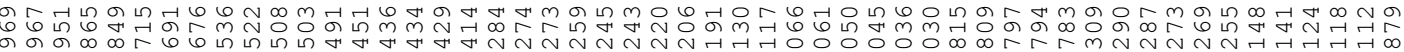

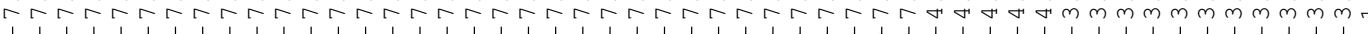<smiles>CC(=O)CC(c1csc2ccccc12)P(=O)(c1ccccc1)c1ccccc1</smiles>
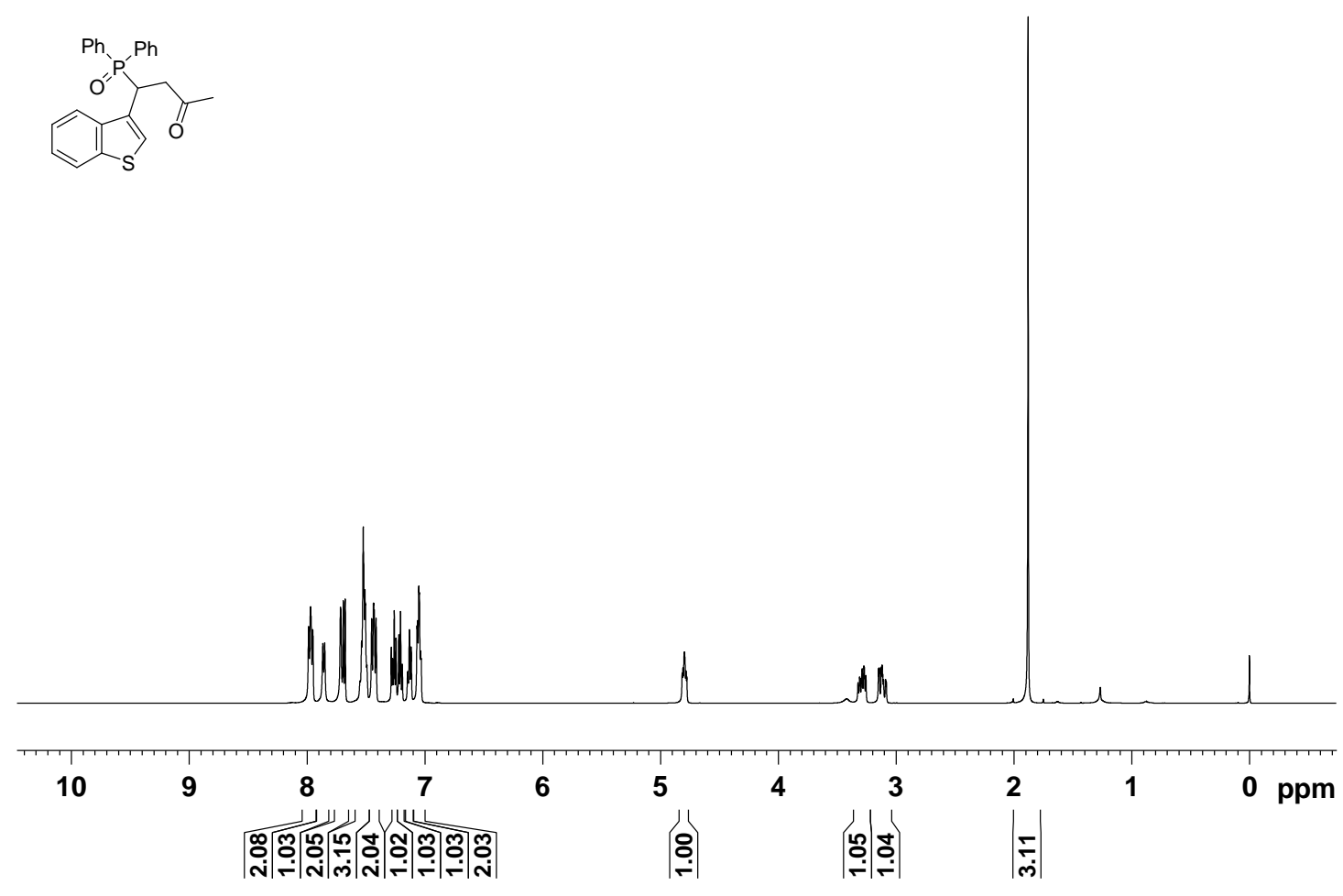

${ }^{13} \mathrm{C} \mathrm{NMR}\left(\mathrm{CDCl}_{3}, 125 \mathrm{MHz}\right)$ of $\mathbf{3 s}$ :
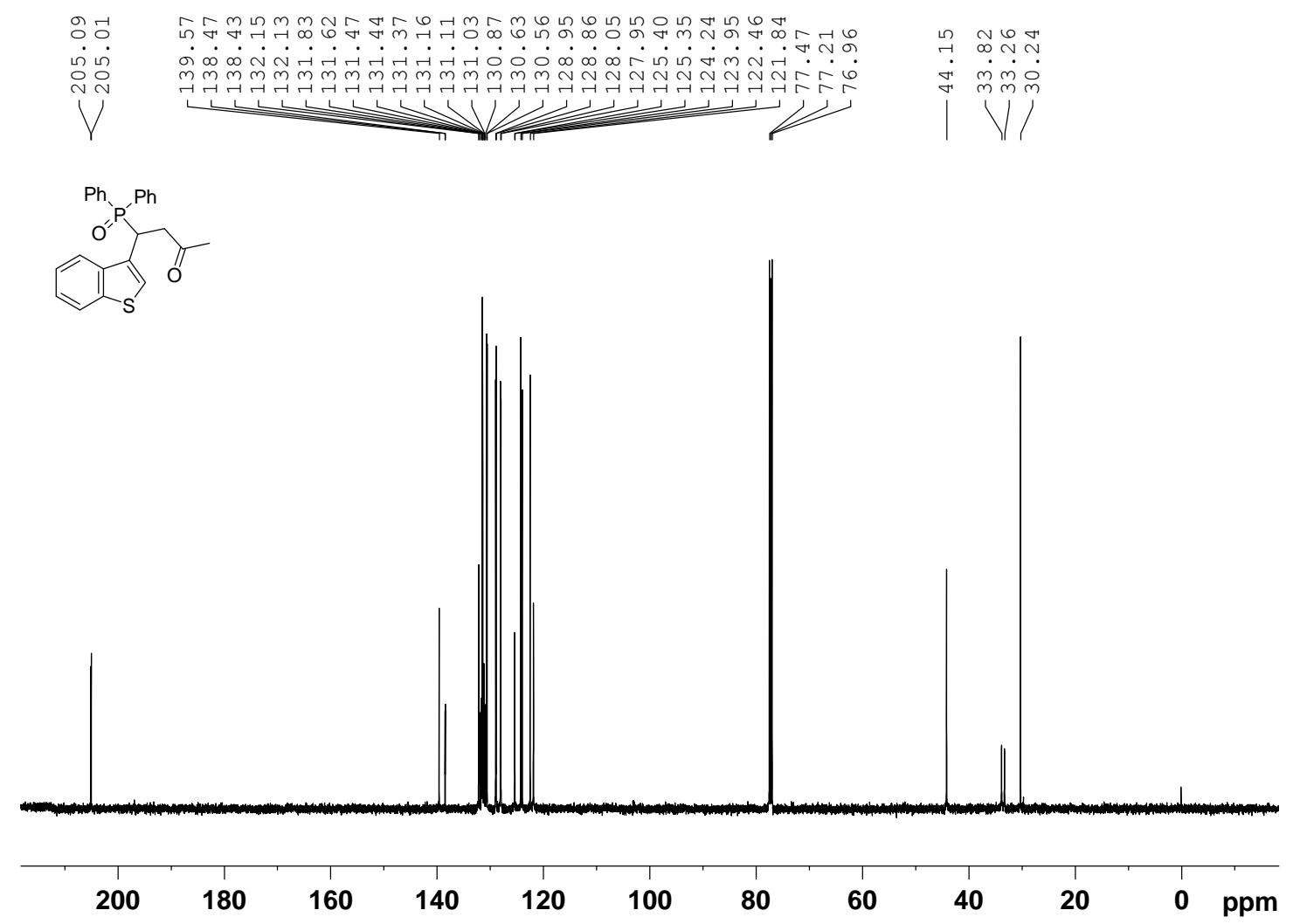
${ }^{31} \mathrm{P} \mathrm{NMR}\left(\mathrm{CDCl}_{3}, 202 \mathrm{MHz}\right)$ of $3 \mathrm{~s}$ :
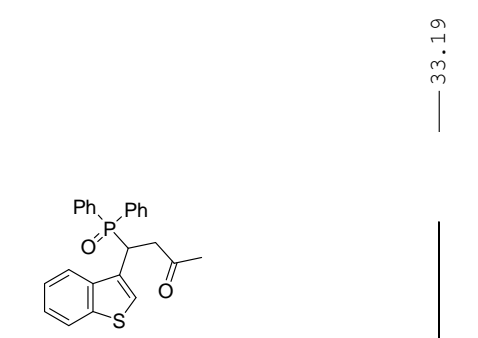

100

50

0

$-50$

$-100$

$-150$

$-200$

ppm

${ }^{1} \mathrm{H} \mathrm{NMR}\left(\mathrm{CDCl}_{3}, 500 \mathrm{MHz}\right)$ of $\mathbf{3 t}$ :

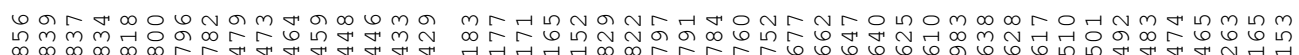

.

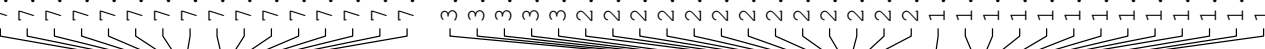

$\longrightarrow$

.
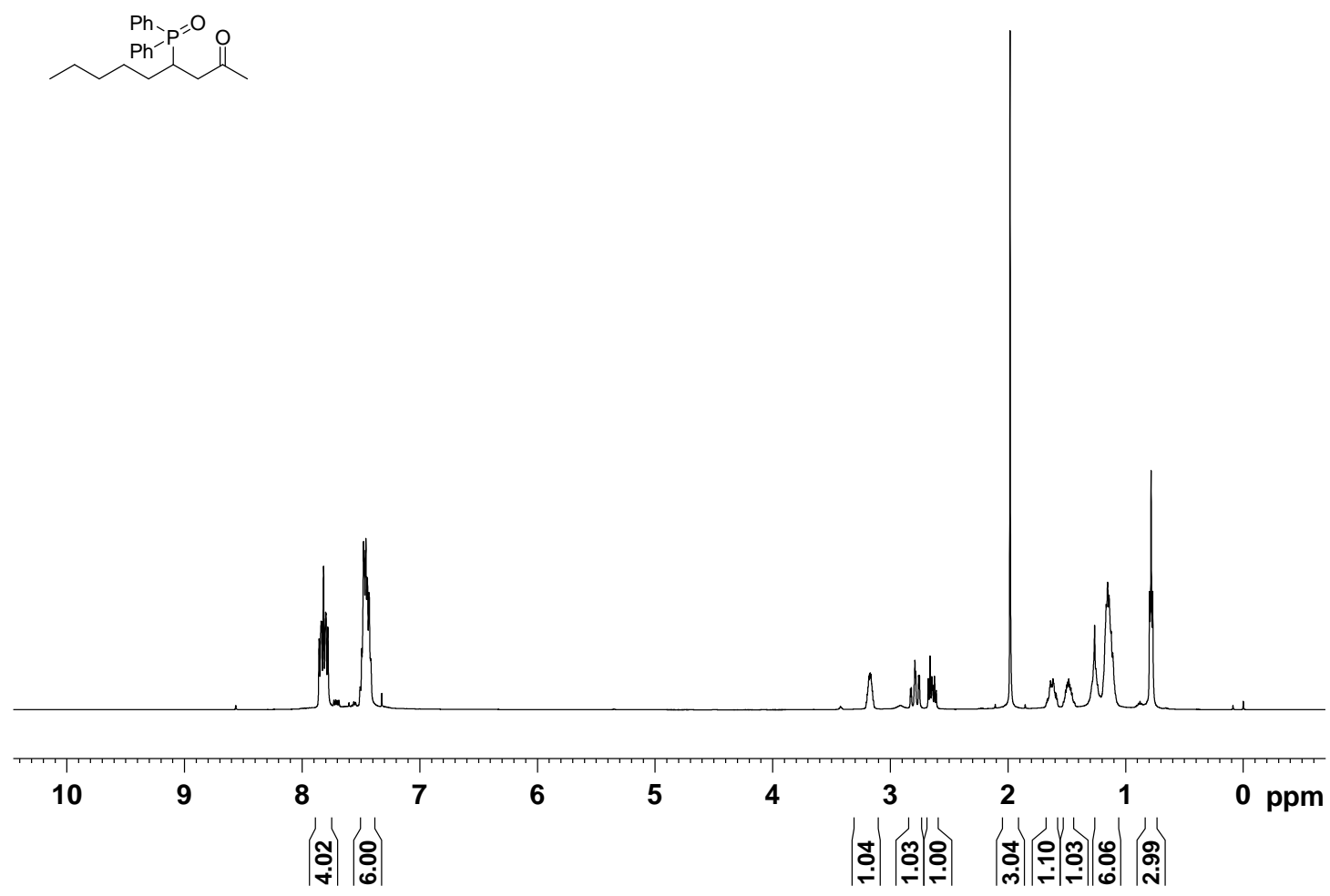


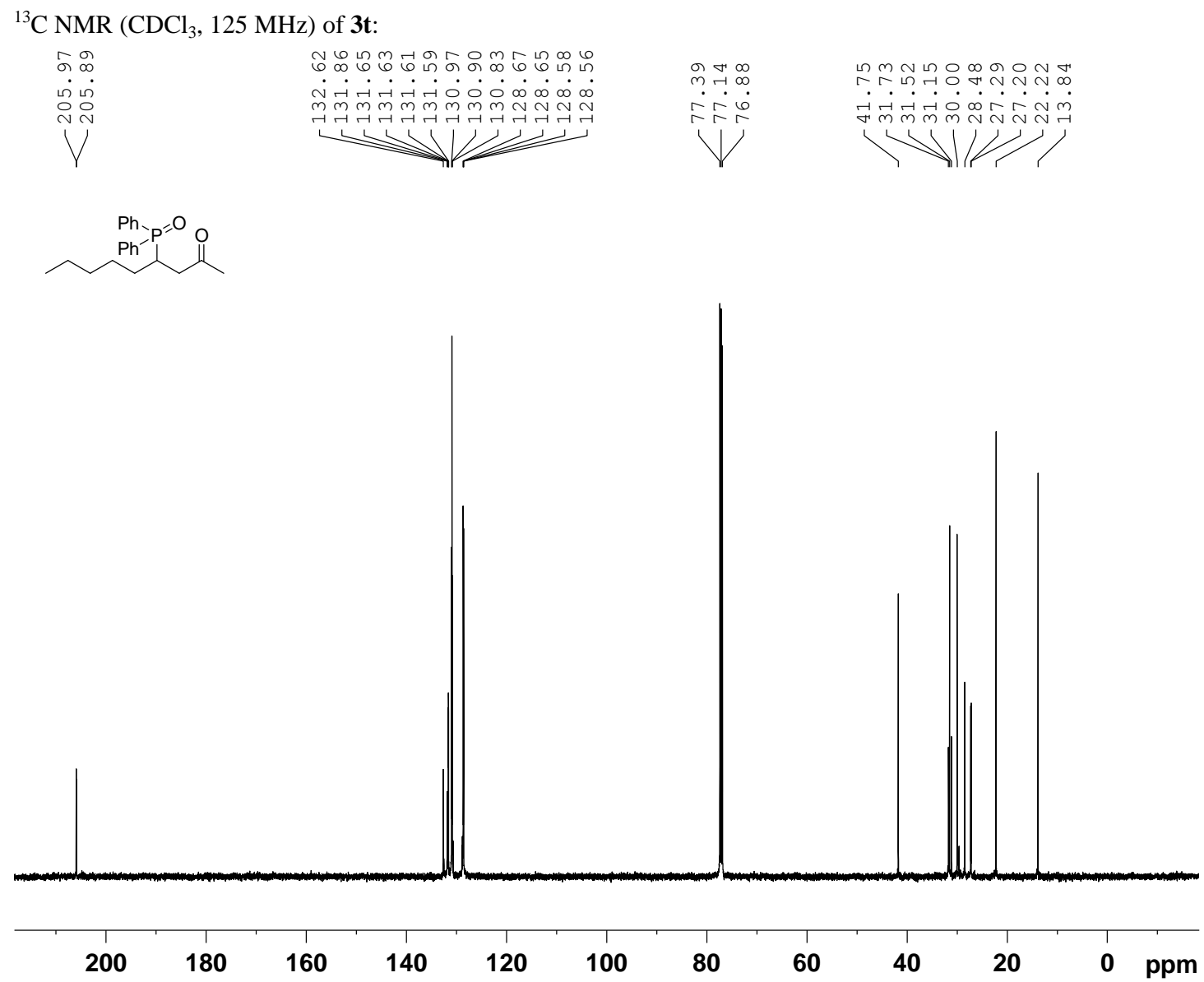

${ }^{31} \mathrm{P} \mathrm{NMR}\left(\mathrm{CDCl}_{3}, 202 \mathrm{MHz}\right)$ of $\mathbf{3 t}$ :
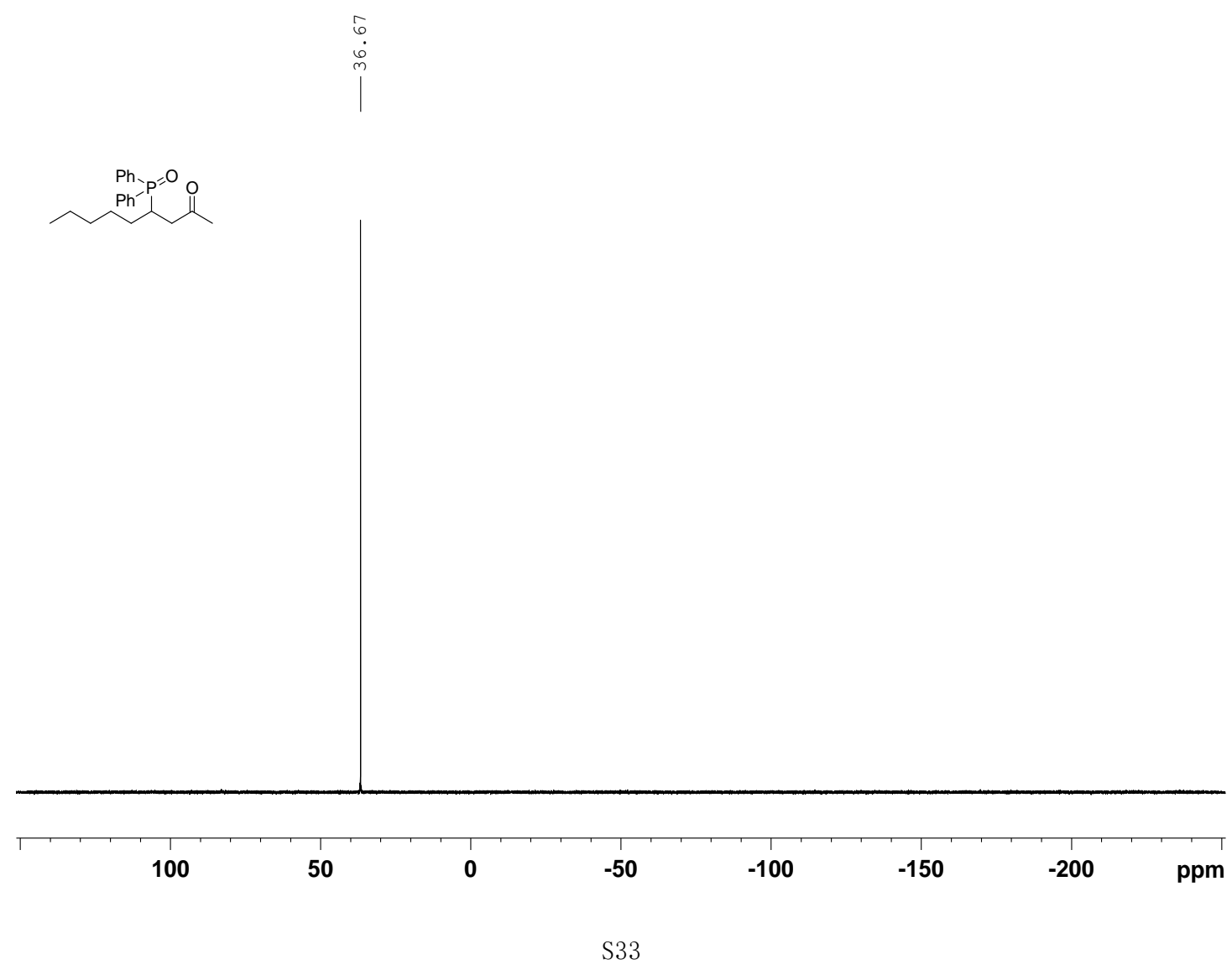
${ }^{1} \mathrm{H}$ NMR $\left(\mathrm{CDCl}_{3}, 500 \mathrm{MHz}\right)$ of $\mathbf{3 u}$ :

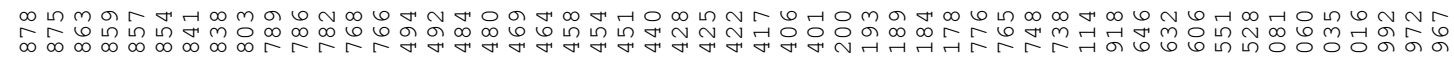

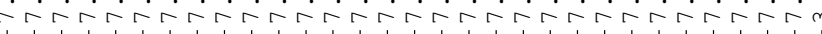

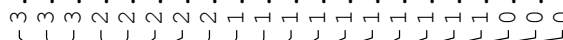
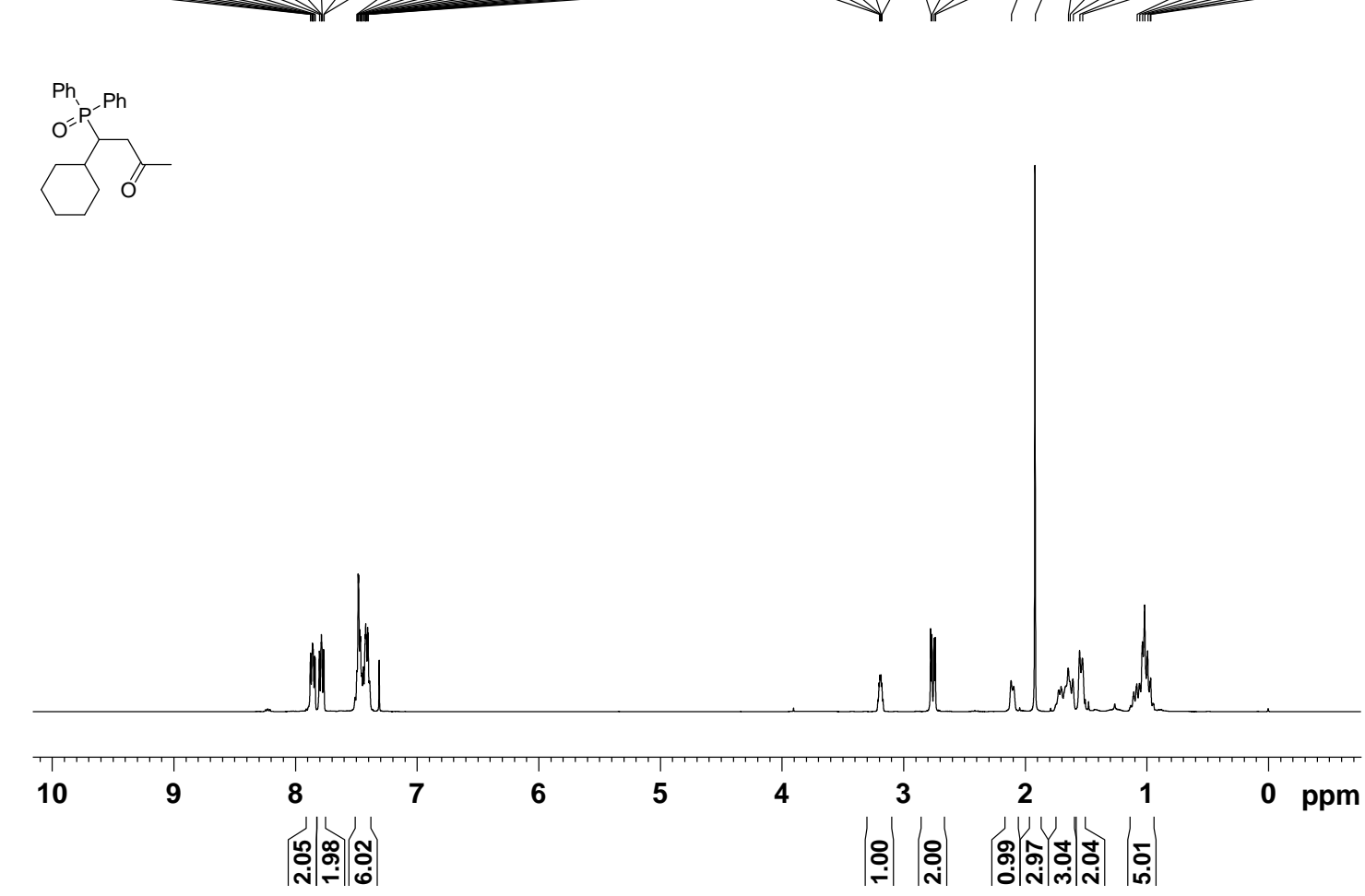

${ }^{13} \mathrm{C} \mathrm{NMR}\left(\mathrm{CDCl}_{3}, 125 \mathrm{MHz}\right)$ of $\mathbf{3 u}$ :
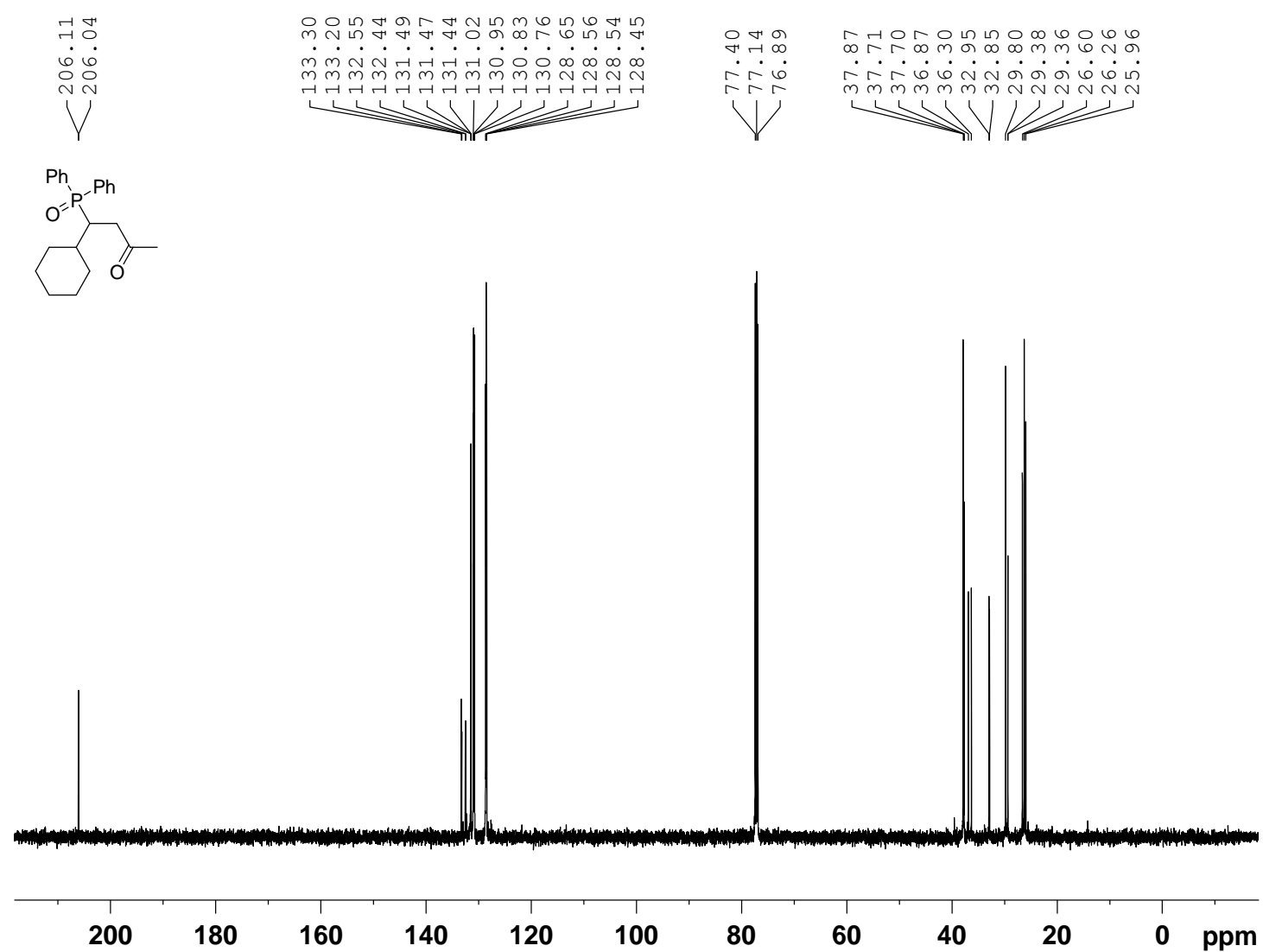
${ }^{31} \mathrm{P}$ NMR $\left(\mathrm{CDCl}_{3}, 202 \mathrm{MHz}\right)$ of $\mathbf{3 u}$ :
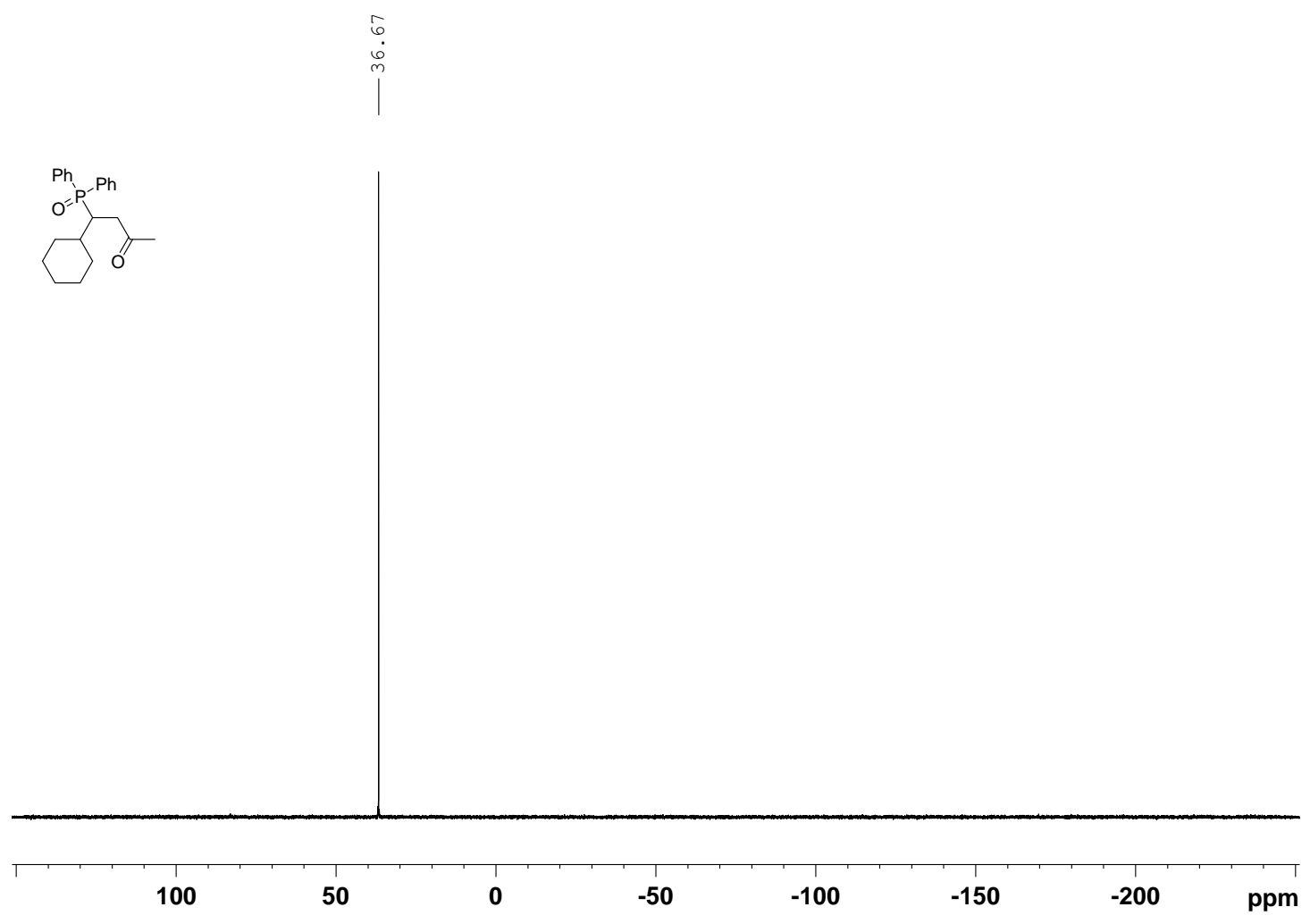

${ }^{1} \mathrm{H} \mathrm{NMR}\left(\mathrm{CDCl}_{3}, 500 \mathrm{MHz}\right)$ of $\mathbf{3 v}$ :

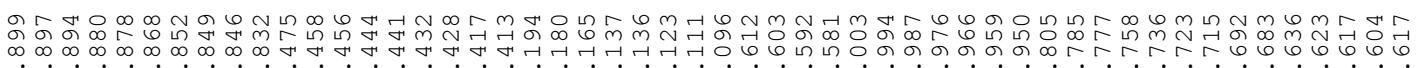

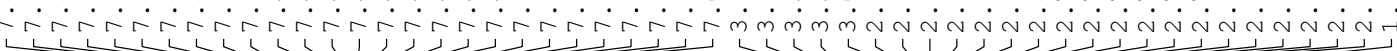

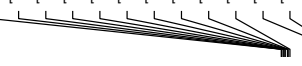<smiles>CC(=O)CC(Cc1ccccc1)P(=O)(O)c1ccccc1</smiles>

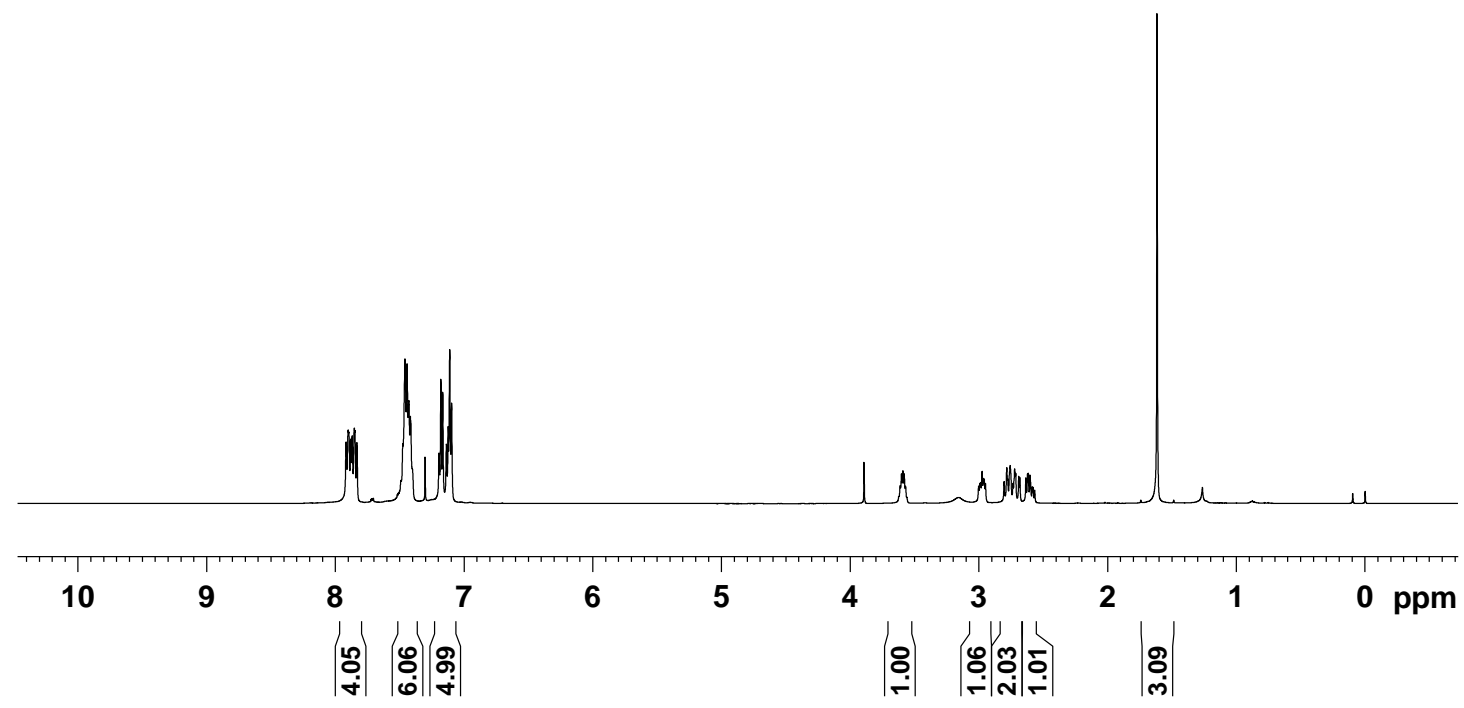


${ }^{13} \mathrm{C}$ NMR $\left(\mathrm{CDCl}_{3}, 125 \mathrm{MHz}\right)$ of $\mathbf{3 v}$ :

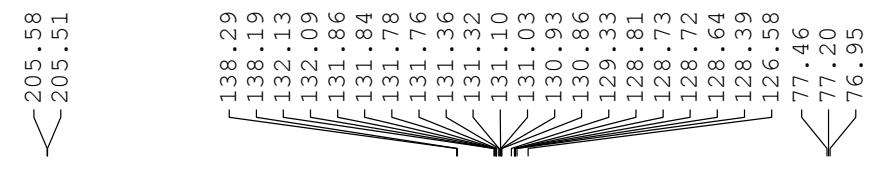

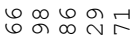

- लंखिं

VI।
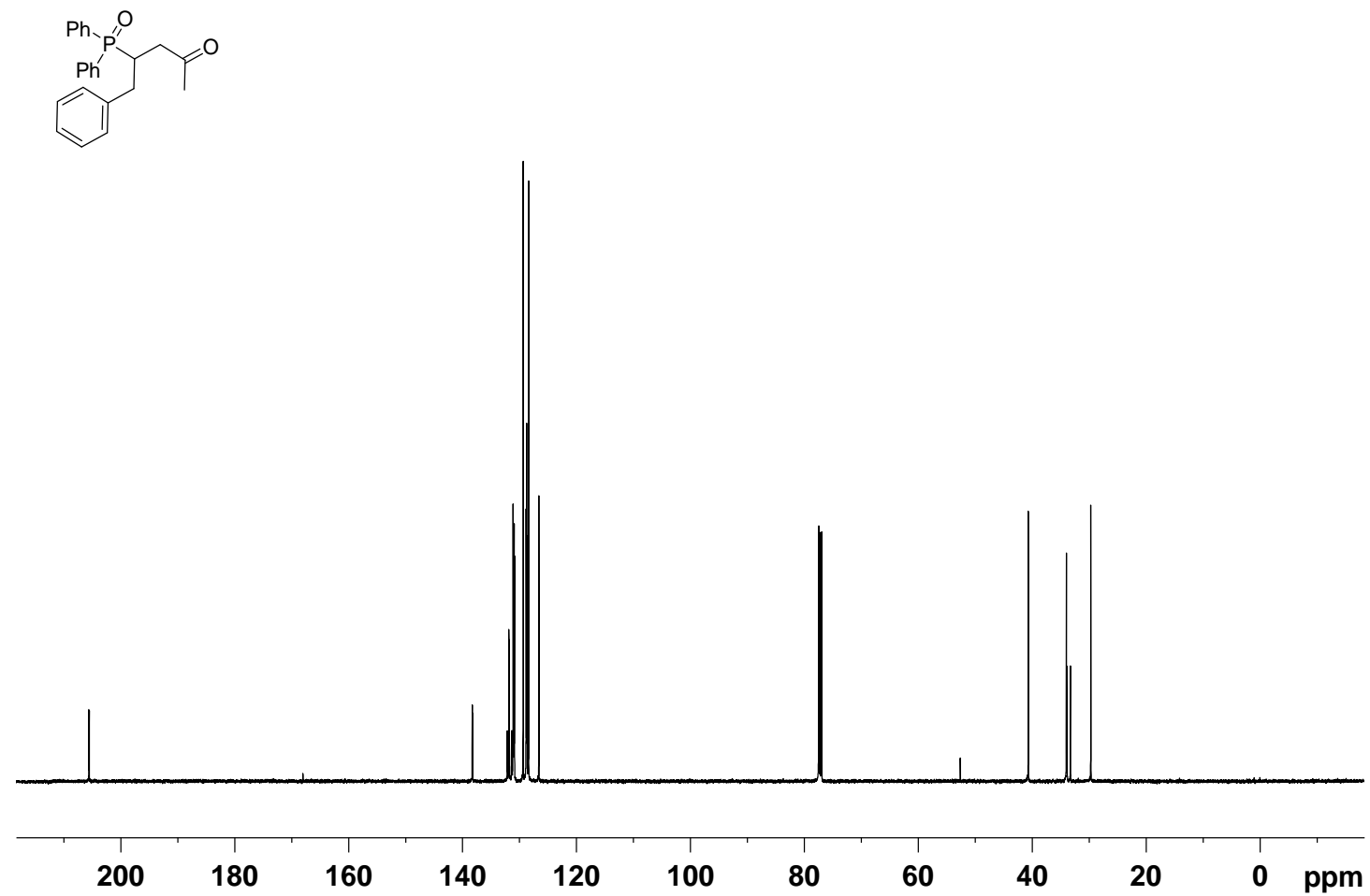

${ }^{31} \mathrm{P} \mathrm{NMR}\left(\mathrm{CDCl}_{3}, 202 \mathrm{MHz}\right)$ of $\mathbf{3 v}$ :
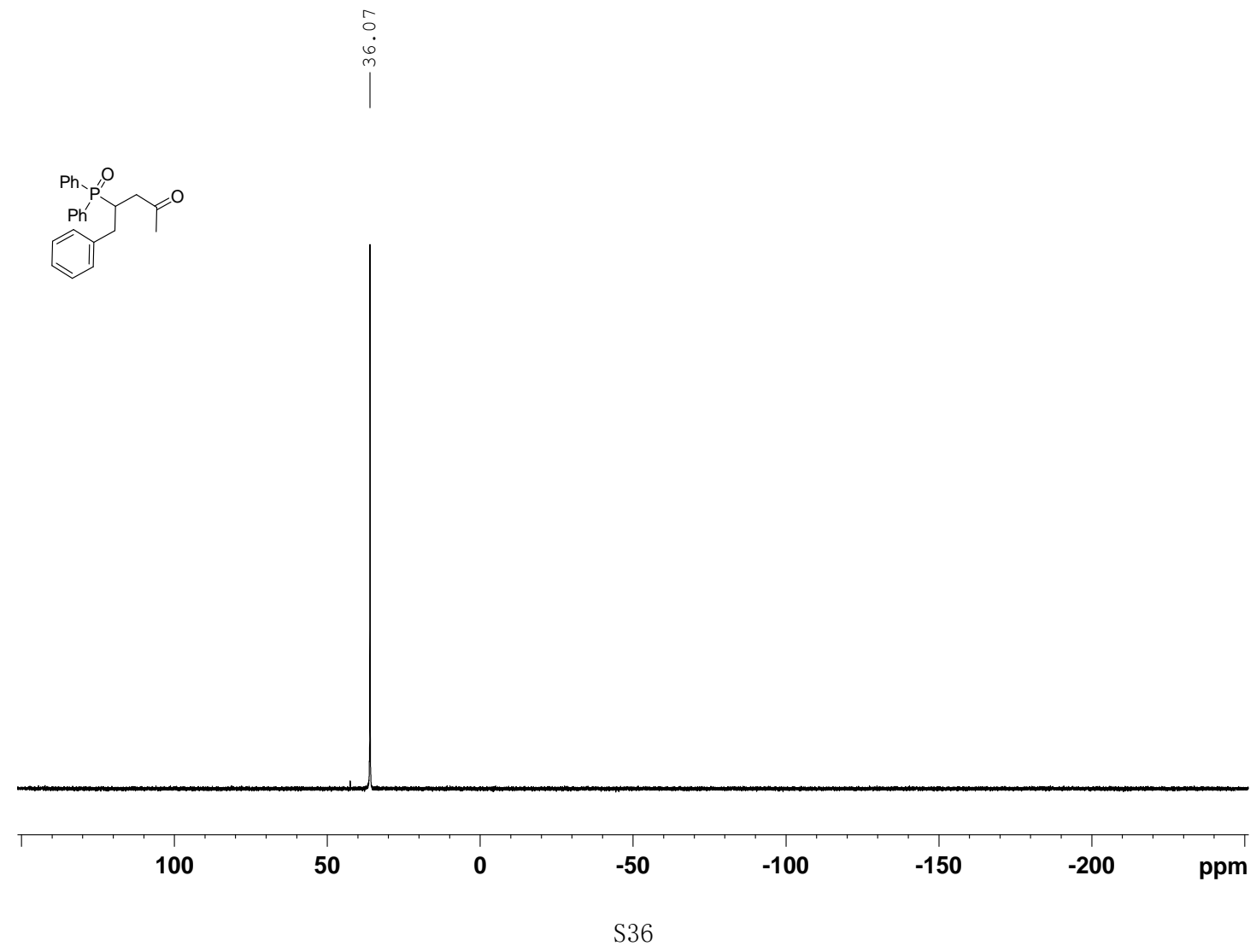
${ }^{1} \mathrm{H}$ NMR $\left(\mathrm{CDCl}_{3}, 500 \mathrm{MHz}\right)$ of $\mathbf{3 w}$ :

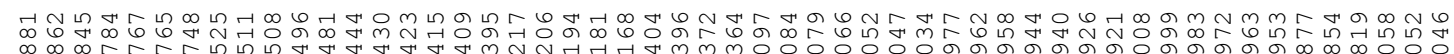
-
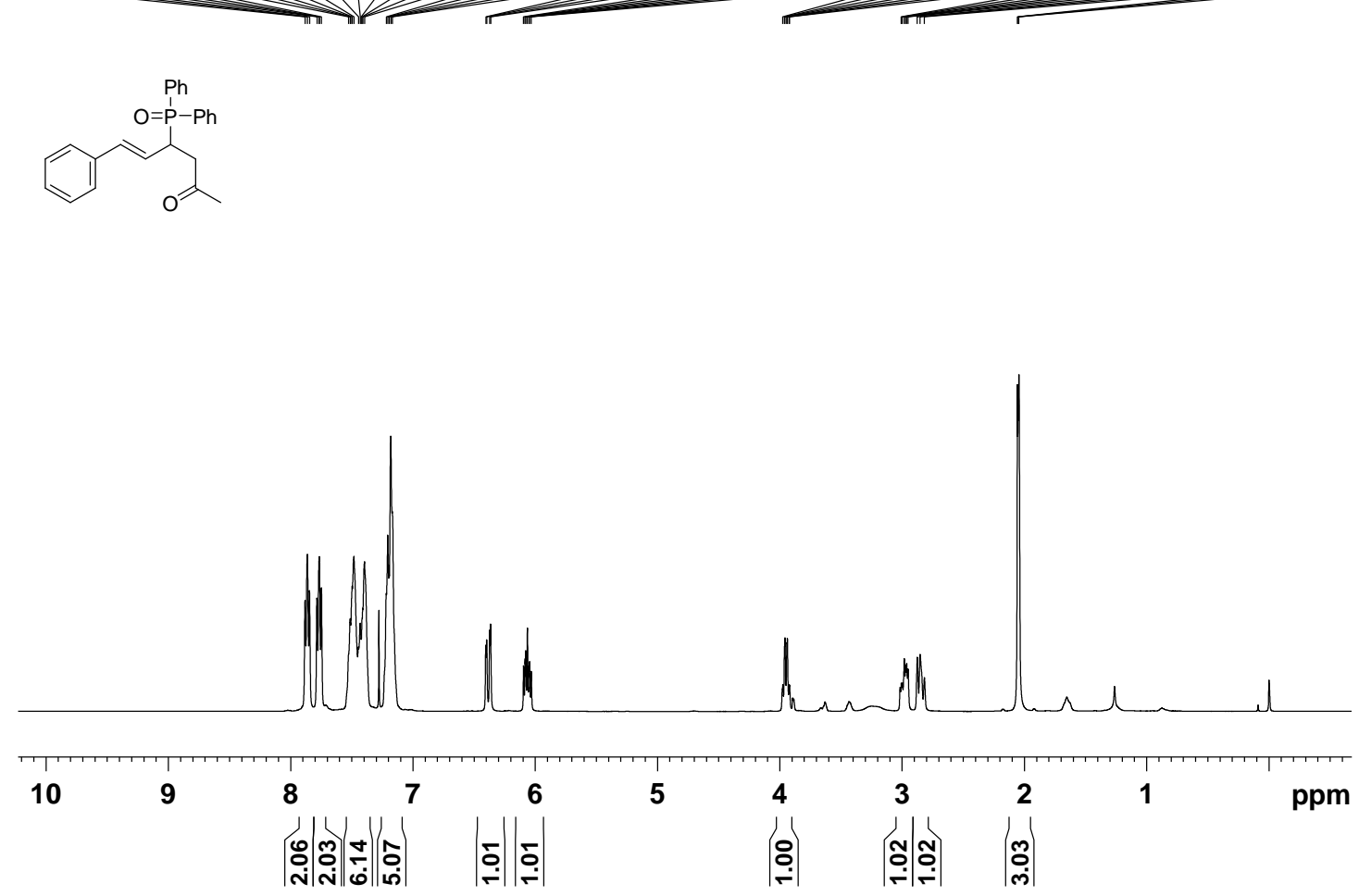

${ }^{13} \mathrm{C}$ NMR $\left(\mathrm{CDCl}_{3}, 125 \mathrm{MHz}\right)$ of $\mathbf{3 w}$ :
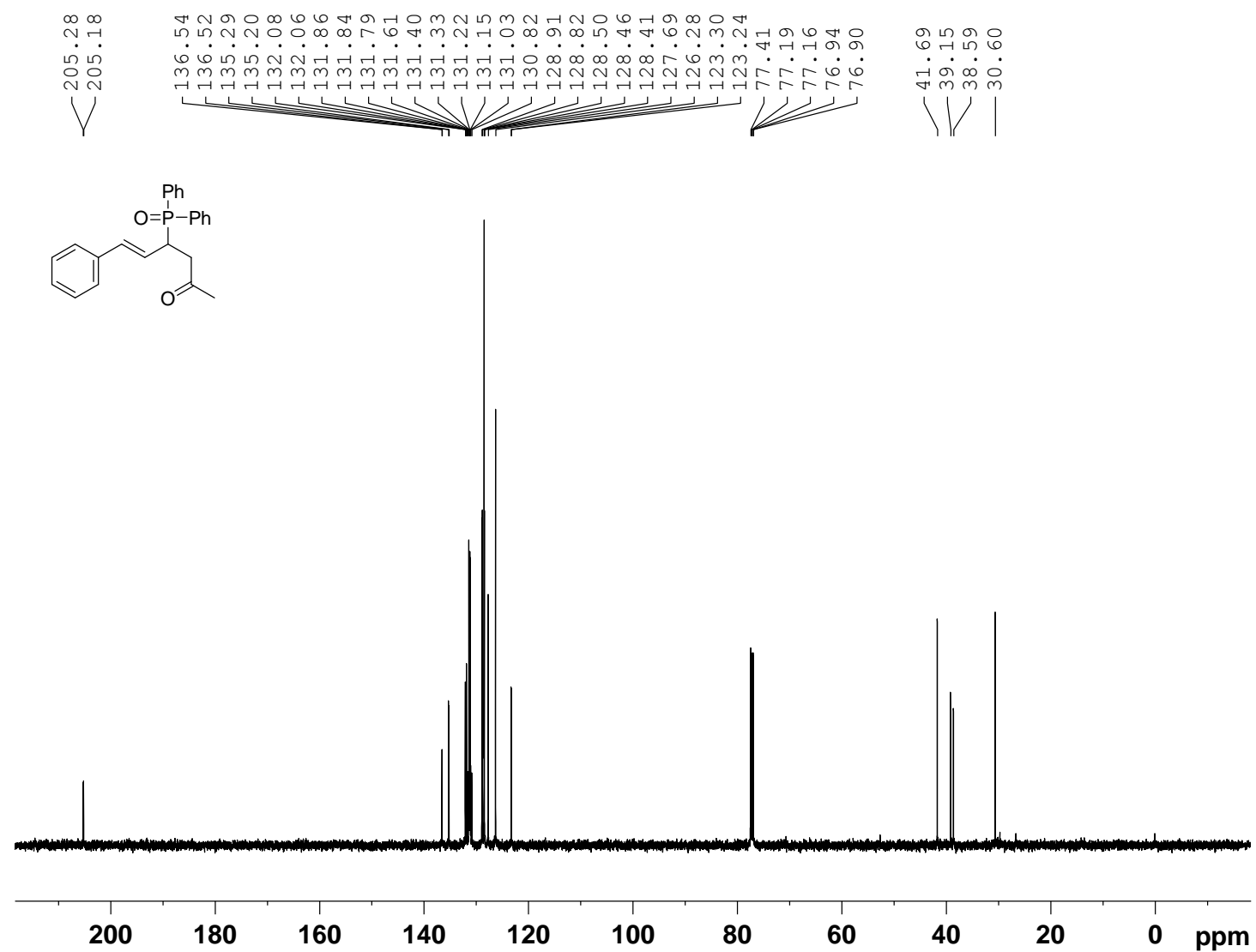
${ }^{31} \mathrm{P}$ NMR $\left(\mathrm{CDCl}_{3}, 202 \mathrm{MHz}\right)$ of $\mathbf{3 w}$ :

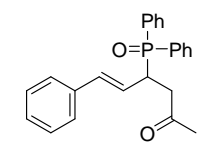

100

50

0

$-50$

$-100$

$-150$

$-200$

ppm

${ }^{1} \mathrm{H} \mathrm{NMR}\left(\mathrm{CDCl}_{3}, 500 \mathrm{MHz}\right)$ of $\mathbf{3 x}$ :

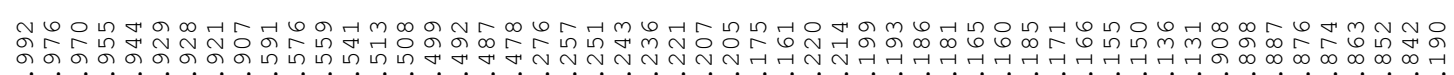

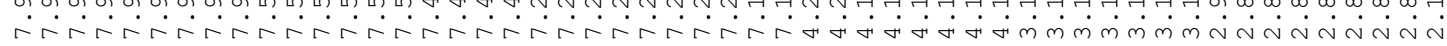

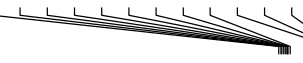<smiles>CC(=O)CC(C#Cc1ccccc1)P(=O)(O)c1ccccc1</smiles>

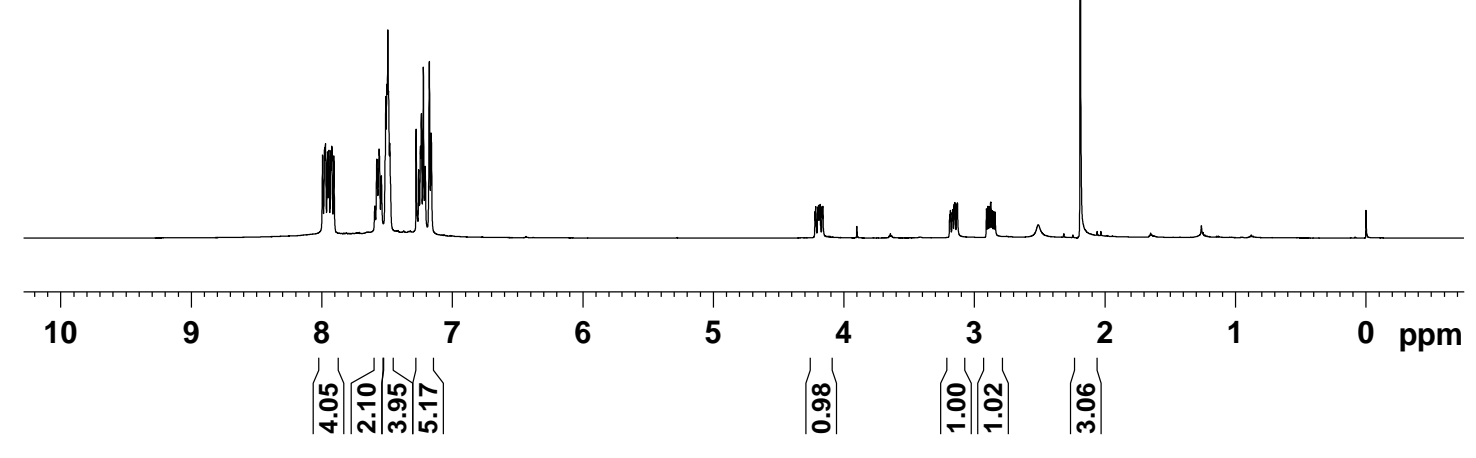


${ }^{13} \mathrm{C}$ NMR $\left(\mathrm{CDCl}_{3}, 125 \mathrm{MHz}\right)$ of $\mathbf{3 x}$ :
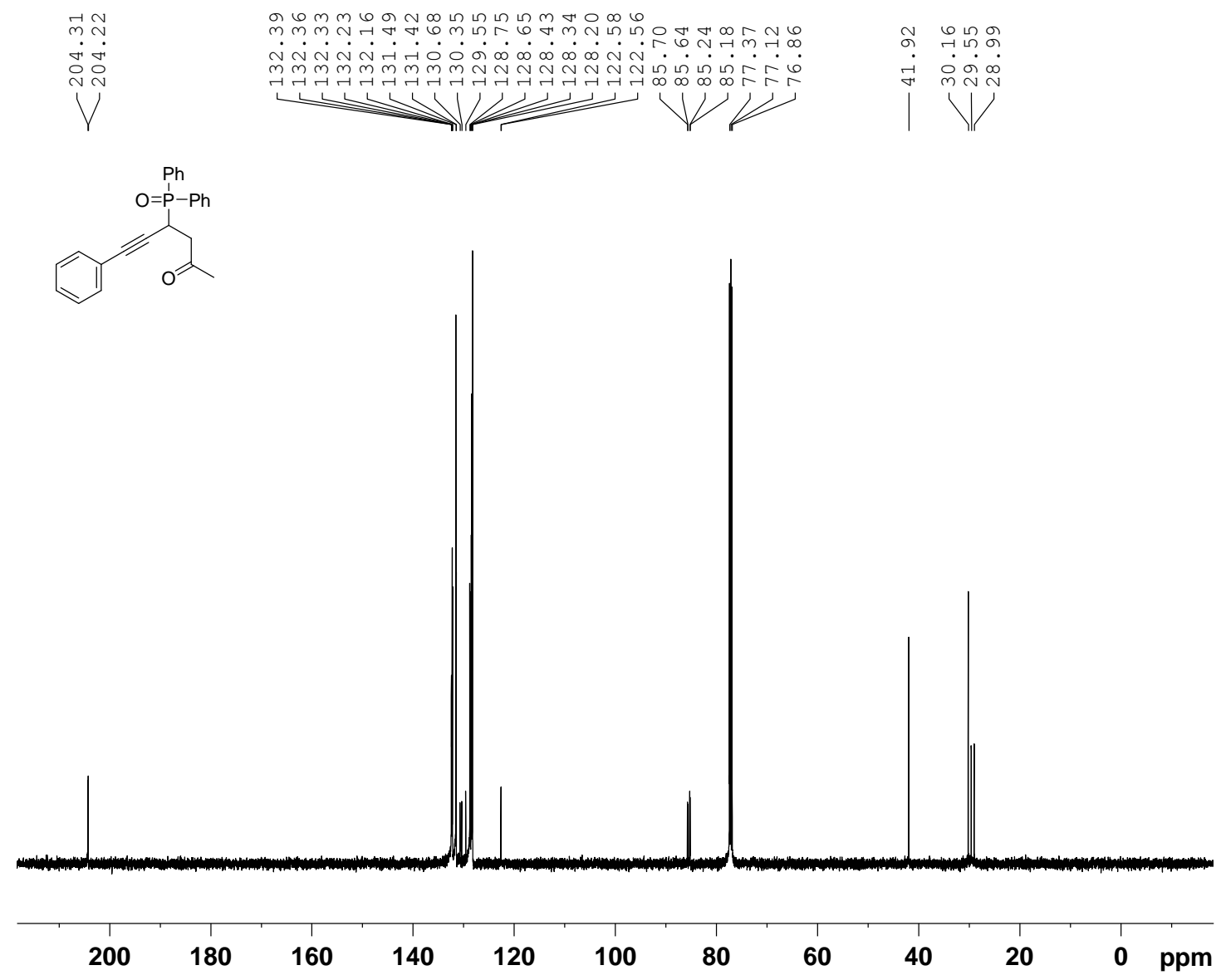

${ }^{31} \mathrm{P}$ NMR $\left(\mathrm{CDCl}_{3}, 202 \mathrm{MHz}\right)$ of $\mathbf{3 x}$ :
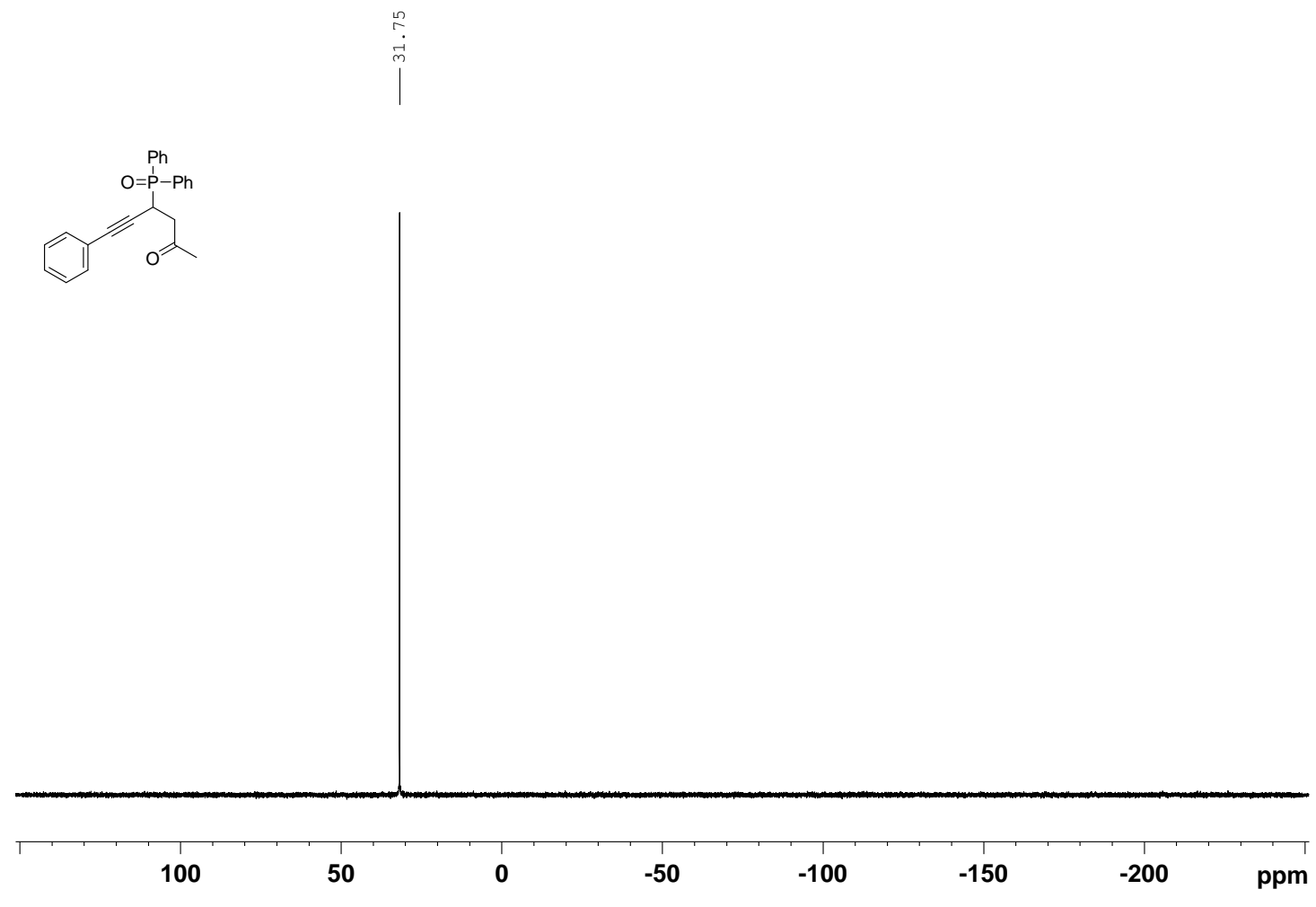
${ }^{1} \mathrm{H}$ NMR $\left(\mathrm{CDCl}_{3}, 500 \mathrm{MHz}\right)$ of $\mathbf{3 y}$ :

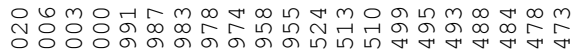

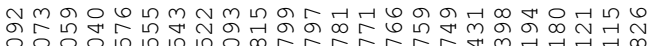

边

$\longrightarrow$
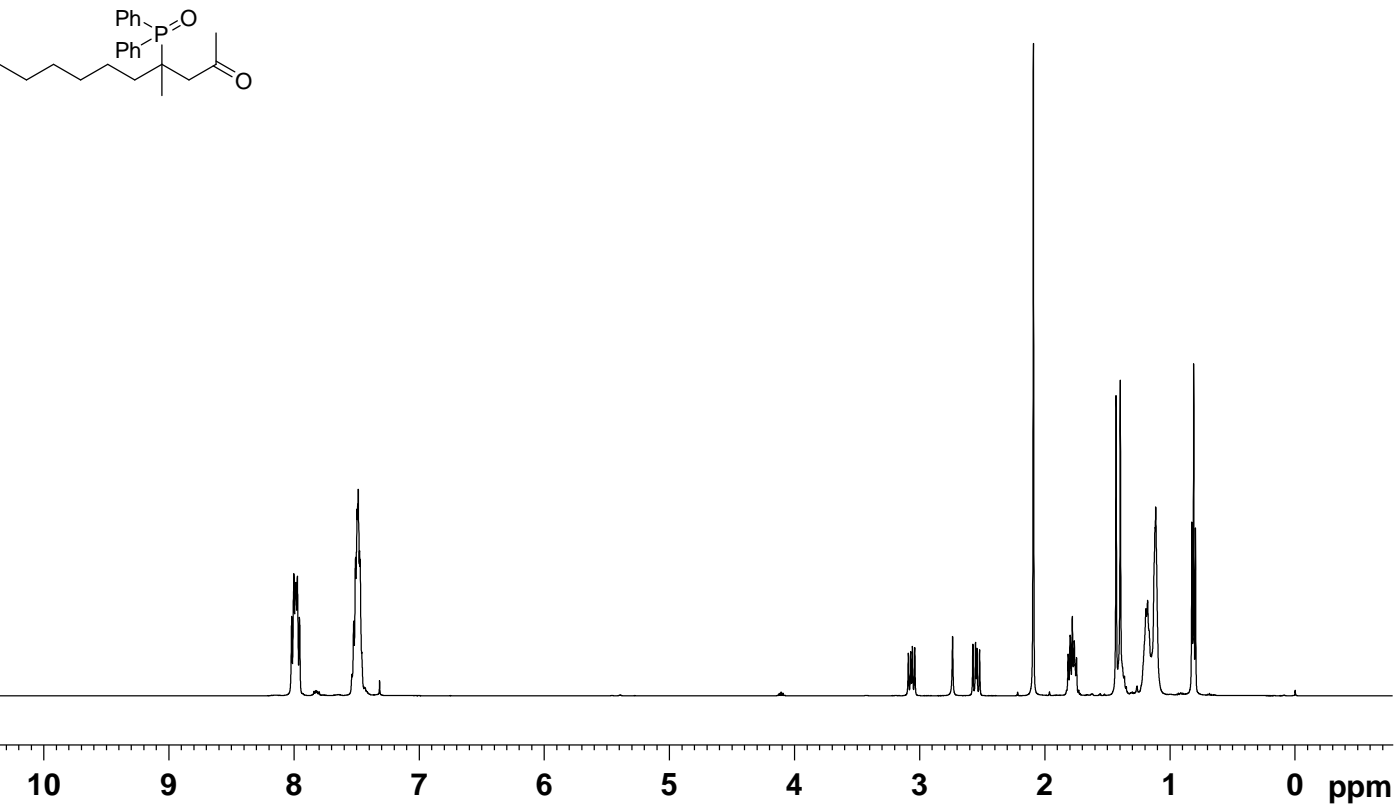
109
$\left|\begin{array}{l}0 \\ \hdashline \\ \dot{\gamma}\end{array}\right|$
|

${ }^{13} \mathrm{C} \mathrm{NMR}\left(\mathrm{CDCl}_{3}, 125 \mathrm{MHz}\right)$ of $\mathbf{3 y}$ :
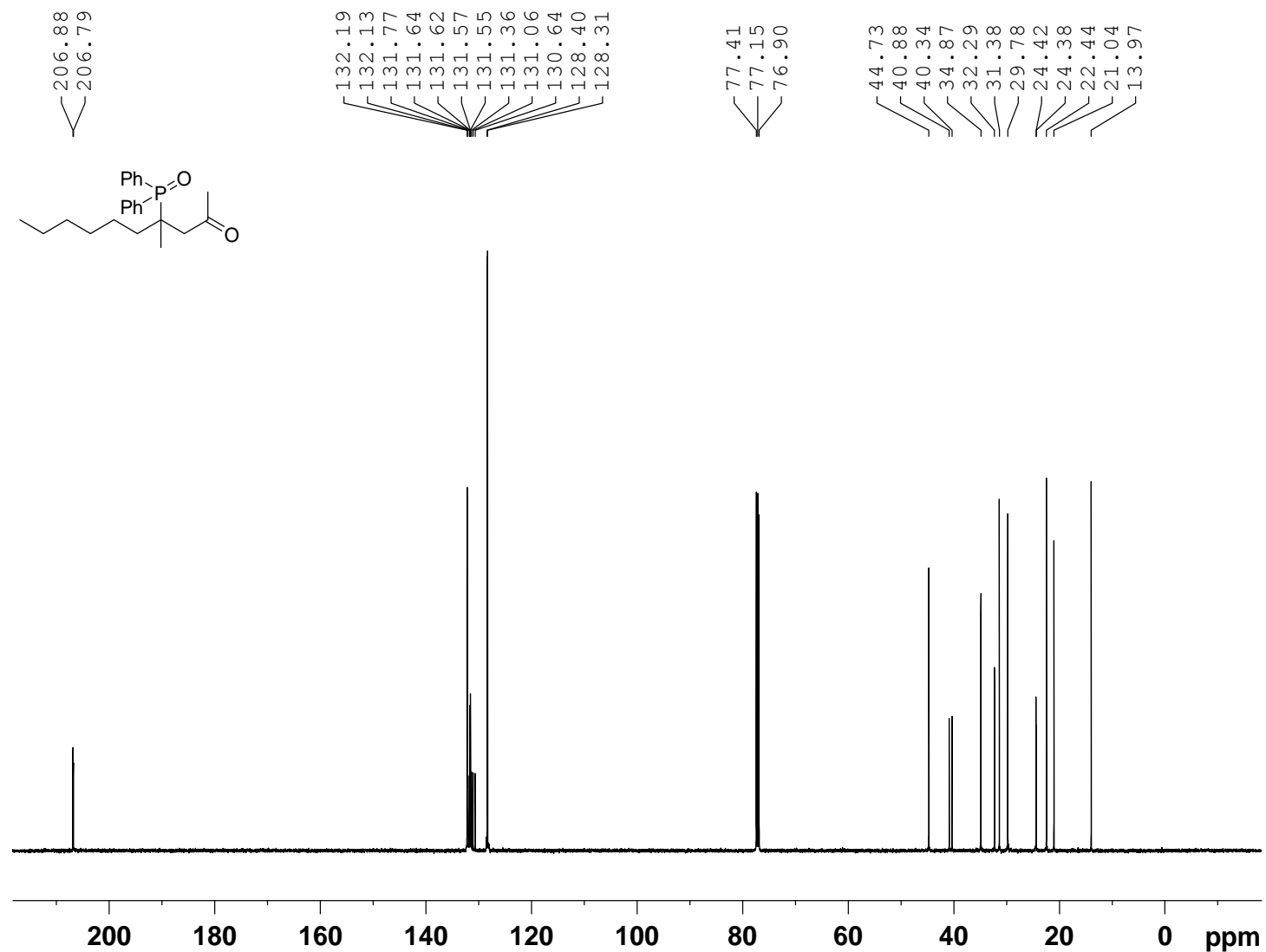
${ }^{31} \mathrm{P} \mathrm{NMR}\left(\mathrm{CDCl}_{3}, 202 \mathrm{MHz}\right)$ of $\mathbf{3 y}$ :
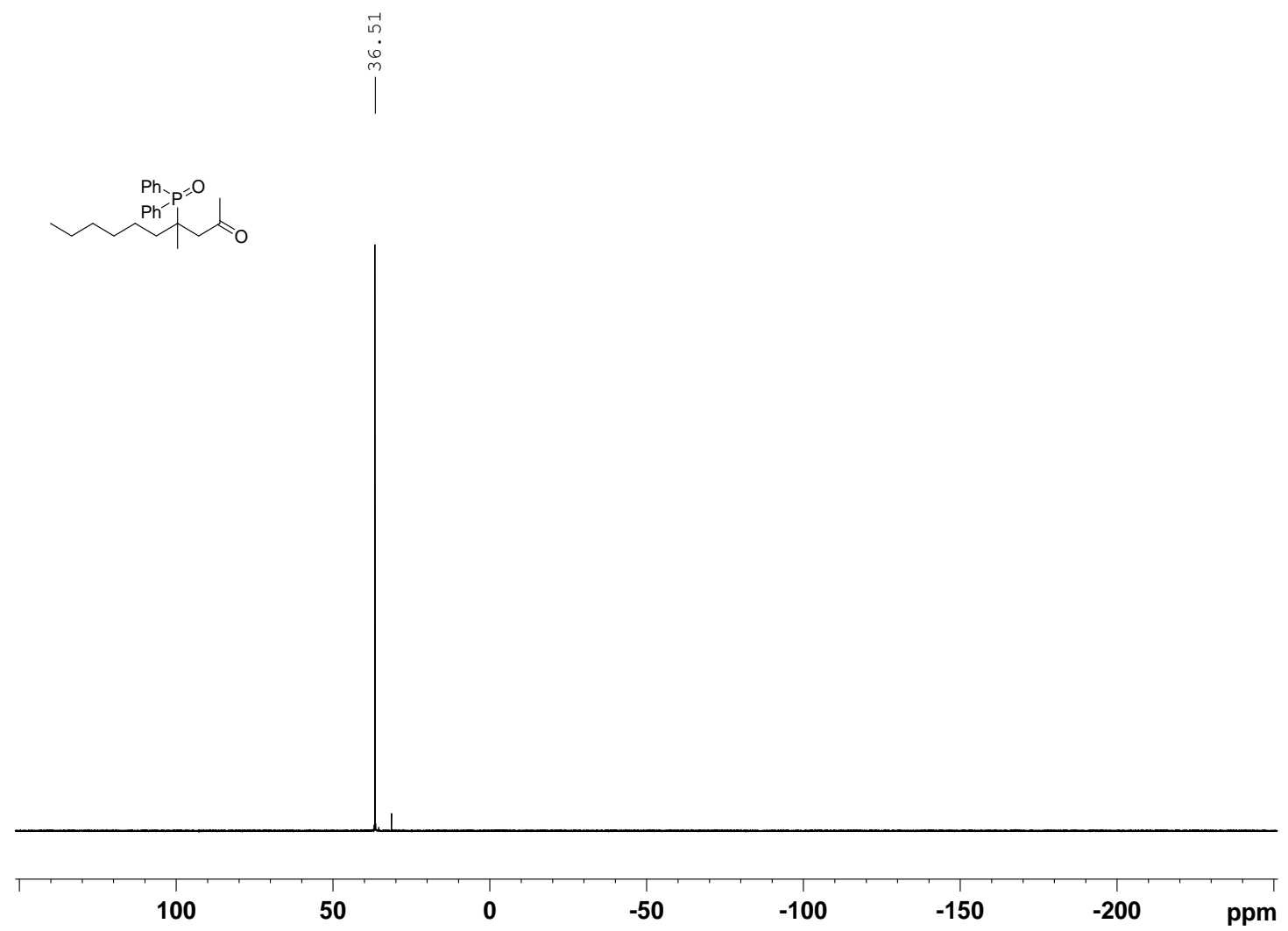

\section{${ }^{1} \mathrm{H} \mathrm{NMR}\left(\mathrm{CDCl}_{3}, 500 \mathrm{MHz}\right)$ of $\mathbf{3 z}$ :}

ाiा

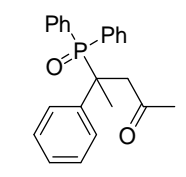


${ }^{13} \mathrm{C}$ NMR $\left(\mathrm{CDCl}_{3}, 125 \mathrm{MHz}\right)$ of $\mathbf{3 z}$ :
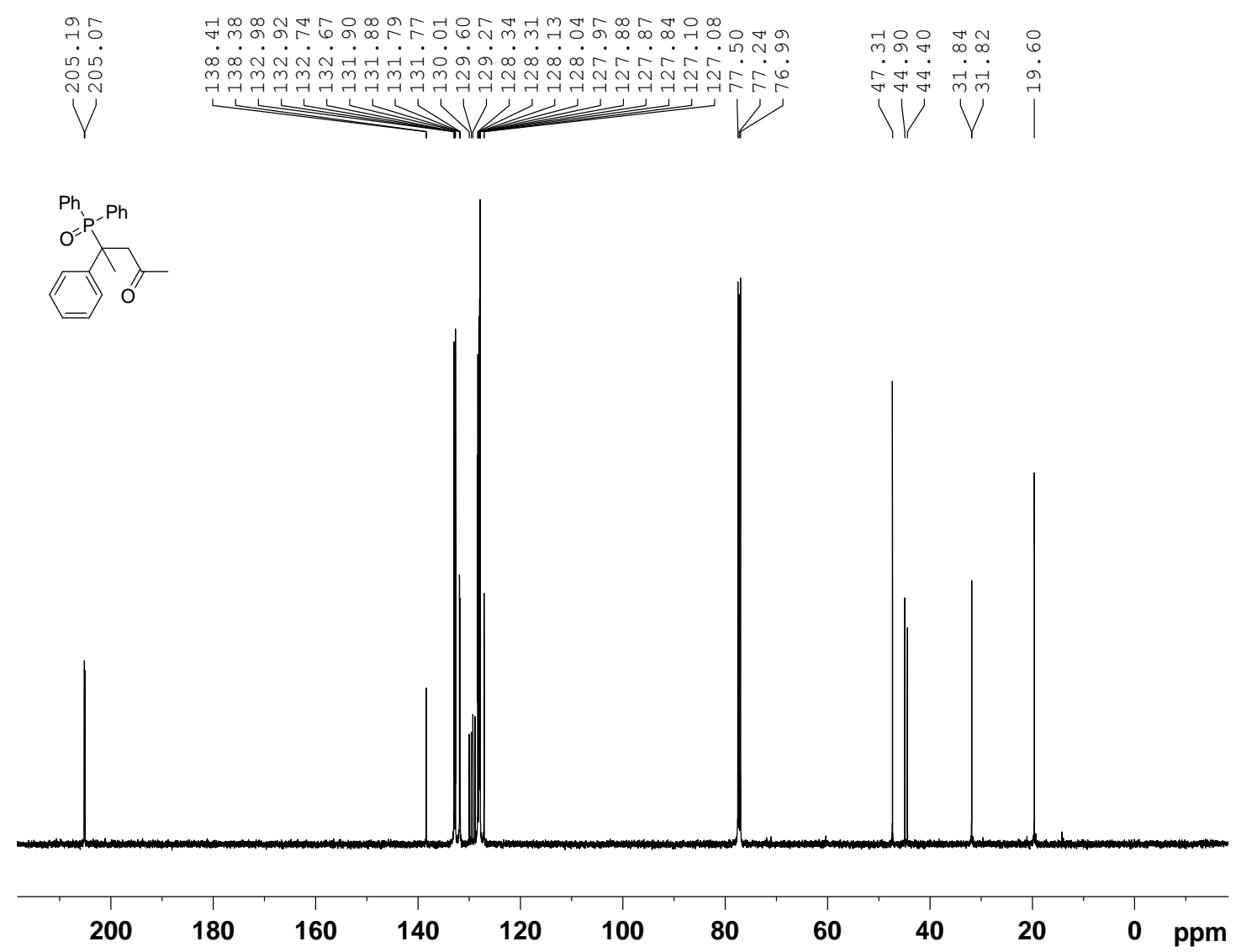

${ }^{31} \mathrm{P} \mathrm{NMR}\left(\mathrm{CDCl}_{3}, 202 \mathrm{MHz}\right)$ of $\mathbf{3 z}$ :
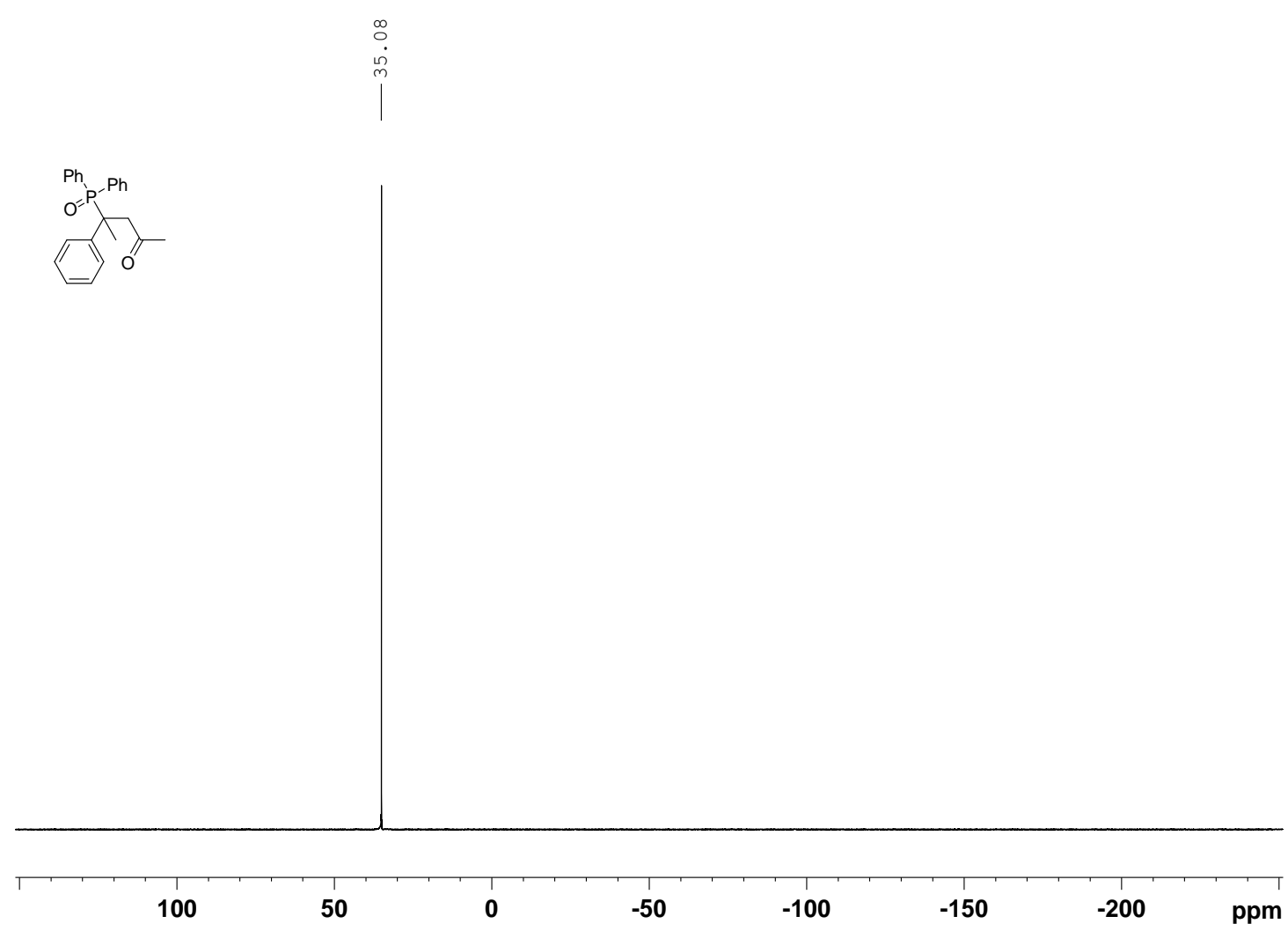
${ }^{1} \mathrm{H} \mathrm{NMR}\left(\mathrm{CDCl}_{3}, 500 \mathrm{MHz}\right)$ of 3aa:
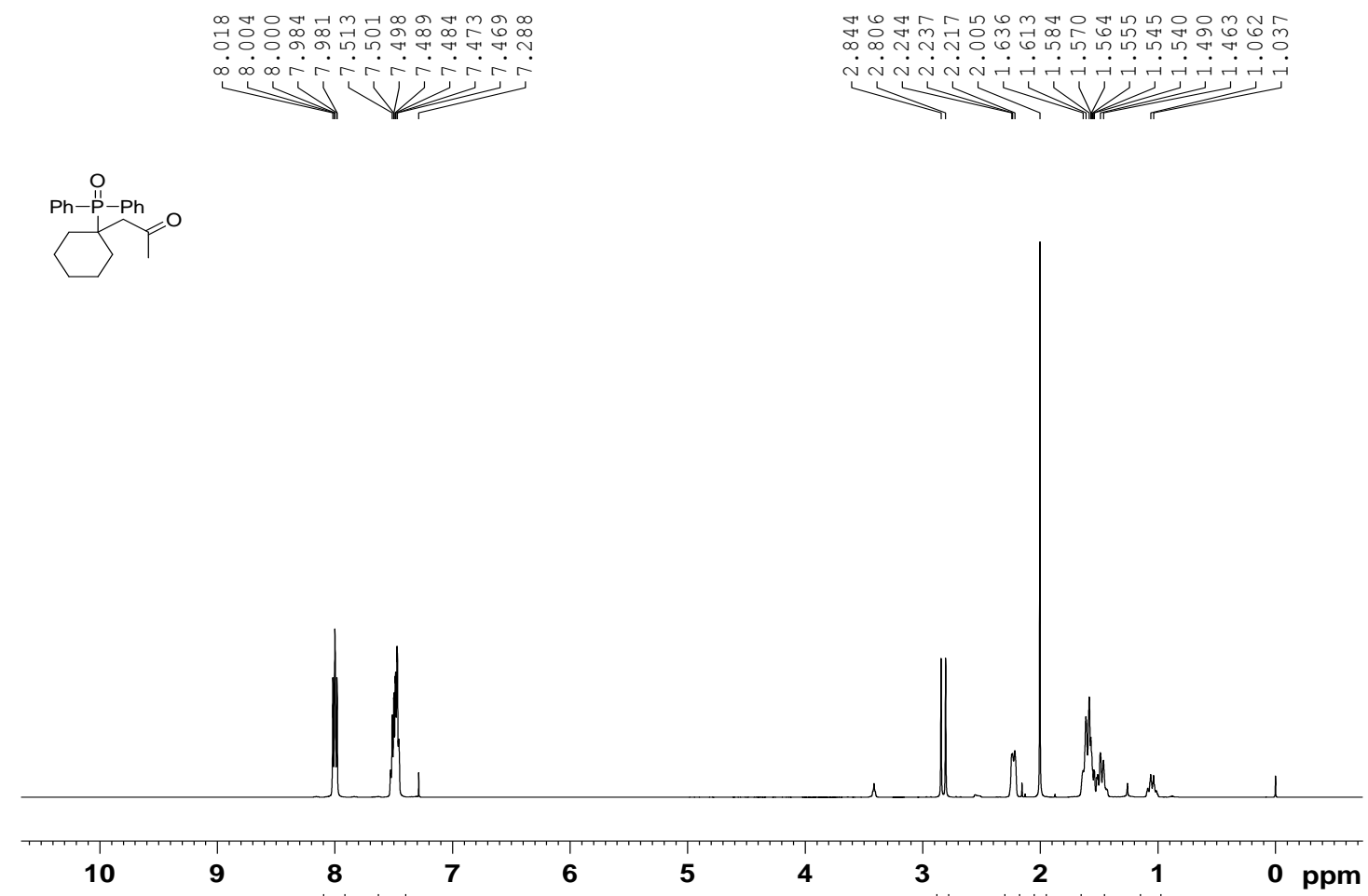

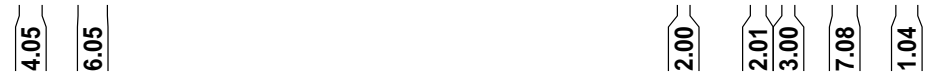

${ }^{13} \mathrm{C} \mathrm{NMR}\left(\mathrm{CDCl}_{3}, 125 \mathrm{MHz}\right)$ of 3aa:

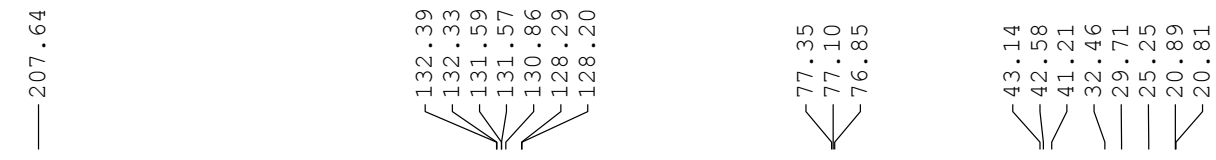

$\overbrace{}^{\text {O }}$

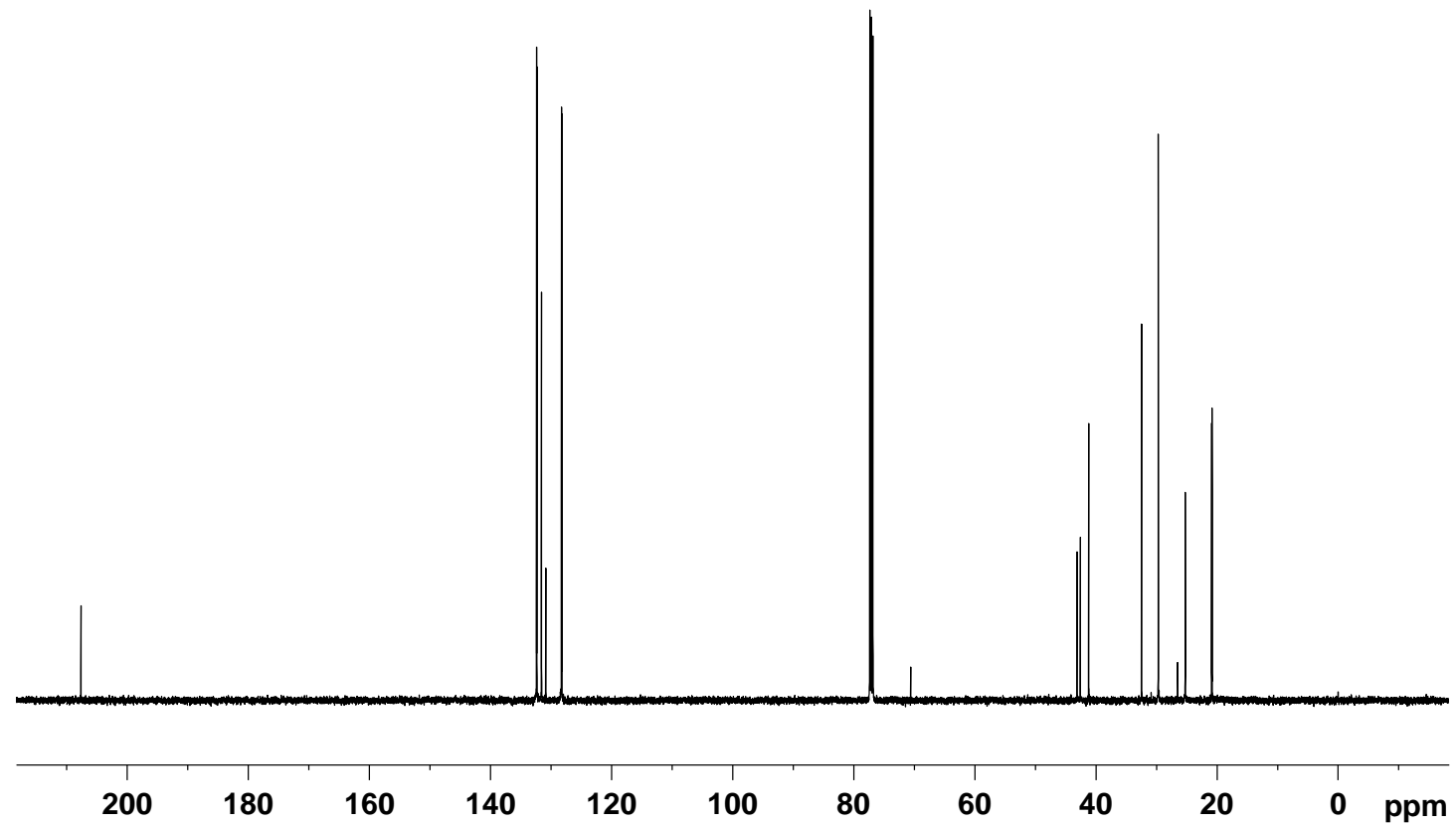


${ }^{31} \mathrm{P}$ NMR $\left(\mathrm{CDCl}_{3}, 202 \mathrm{MHz}\right)$ of 3aa:
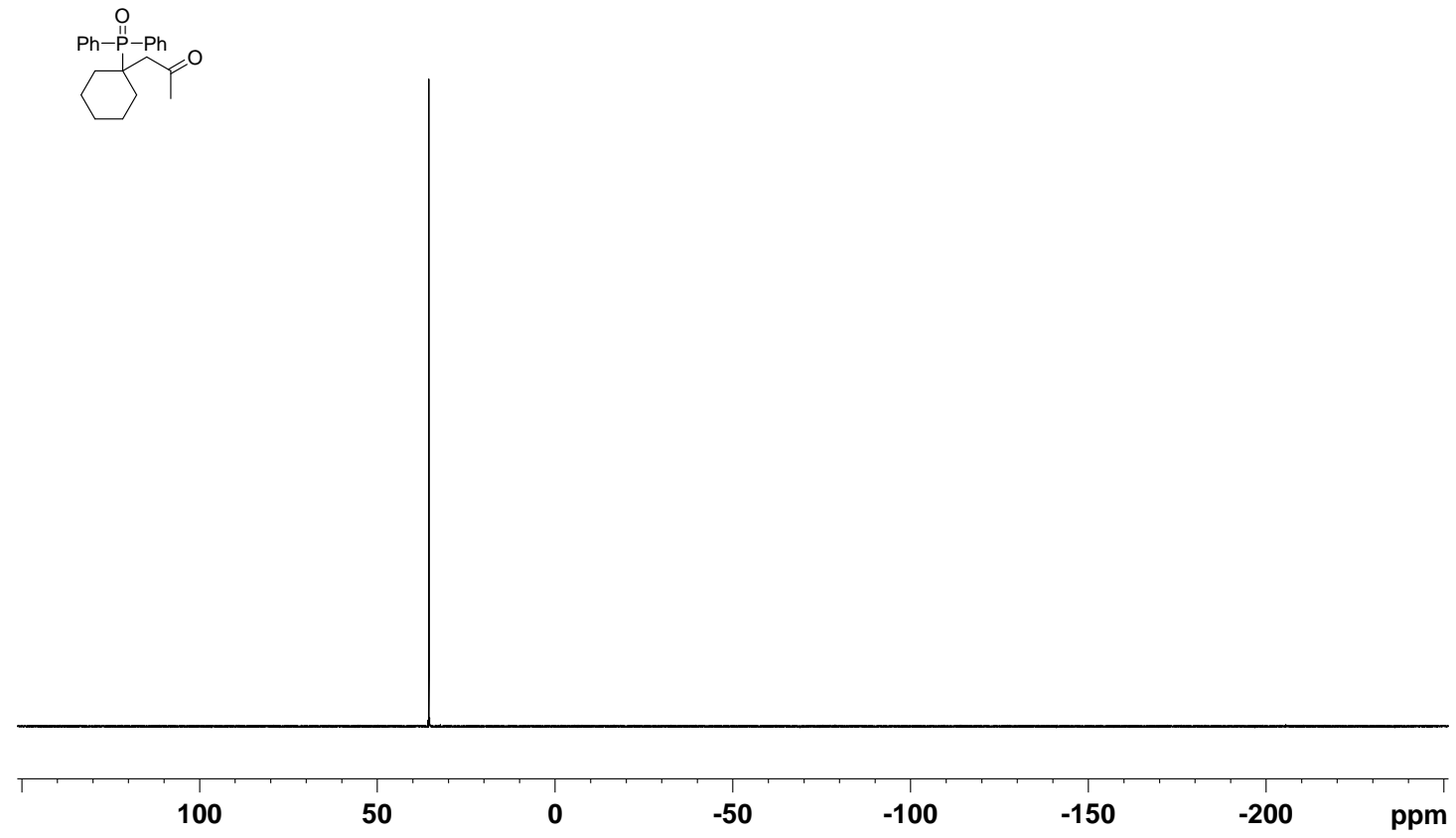

${ }^{1} \mathrm{H} \mathrm{NMR}\left(\mathrm{CDCl}_{3}, 500 \mathrm{MHz}\right)$ of $\mathbf{3 a b}$ :

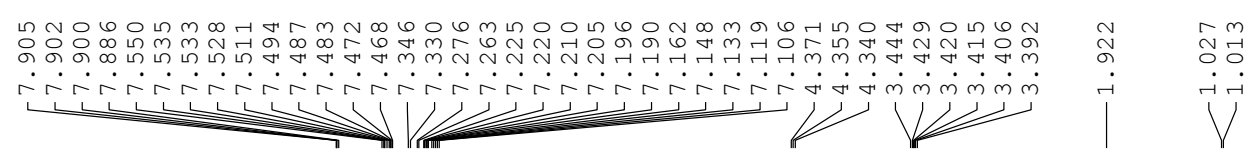
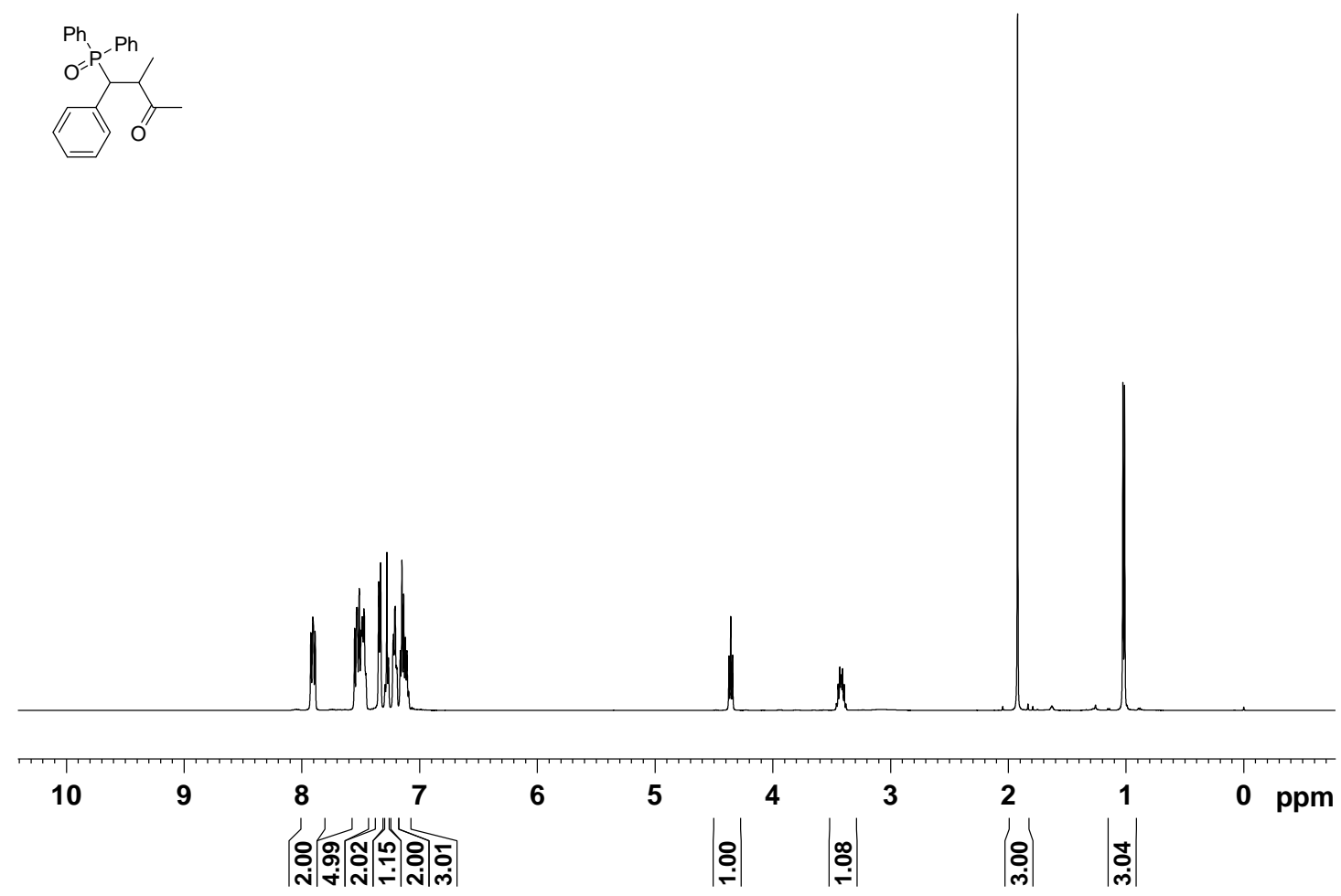
${ }^{13} \mathrm{C} \mathrm{NMR}\left(\mathrm{CDCl}_{3}, 125 \mathrm{MHz}\right)$ of $\mathbf{3 a b}:$
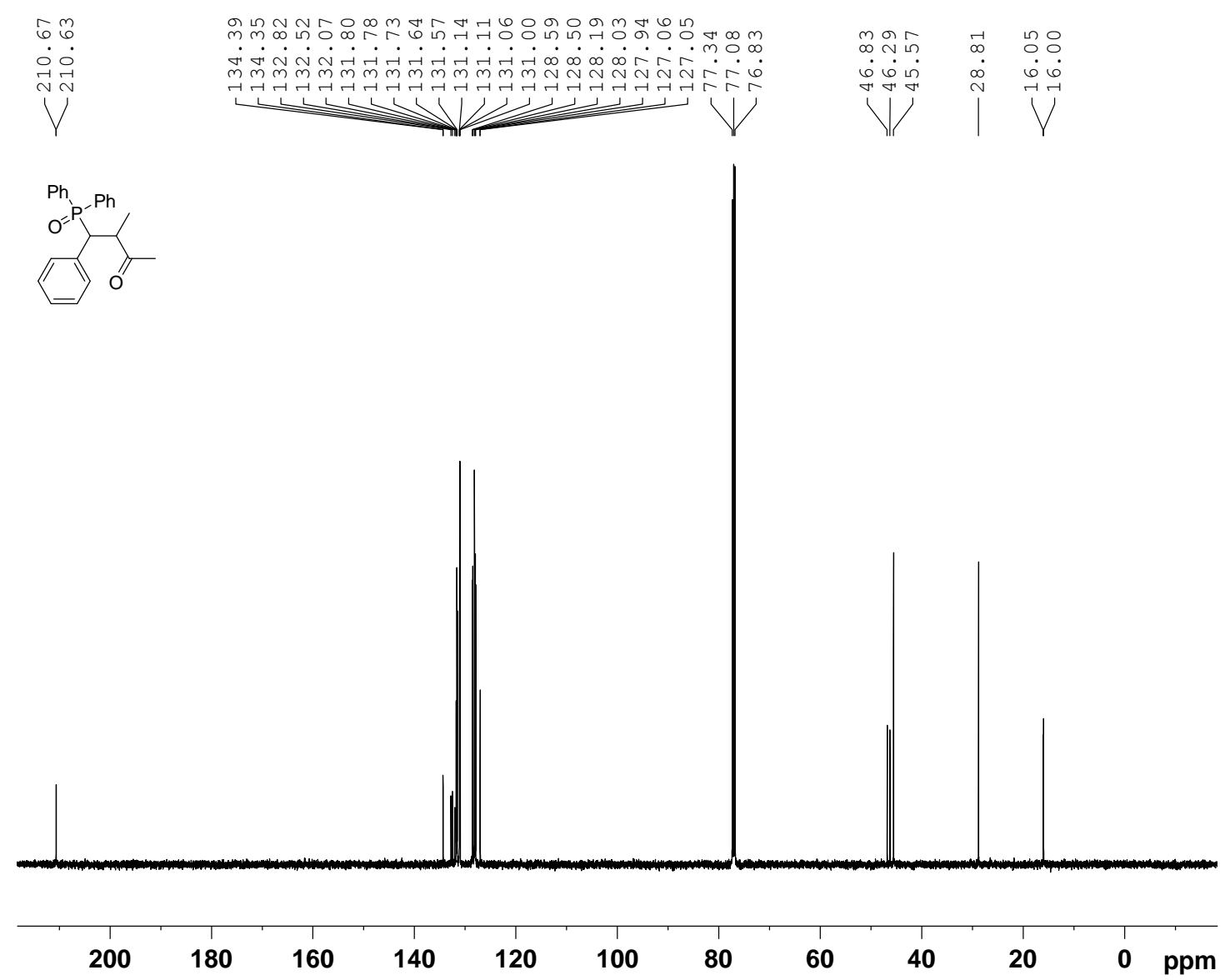

${ }^{31} \mathrm{P}$ NMR $\left(\mathrm{CDCl}_{3}, 202 \mathrm{MHz}\right)$ of $\mathbf{3 a b}$ :
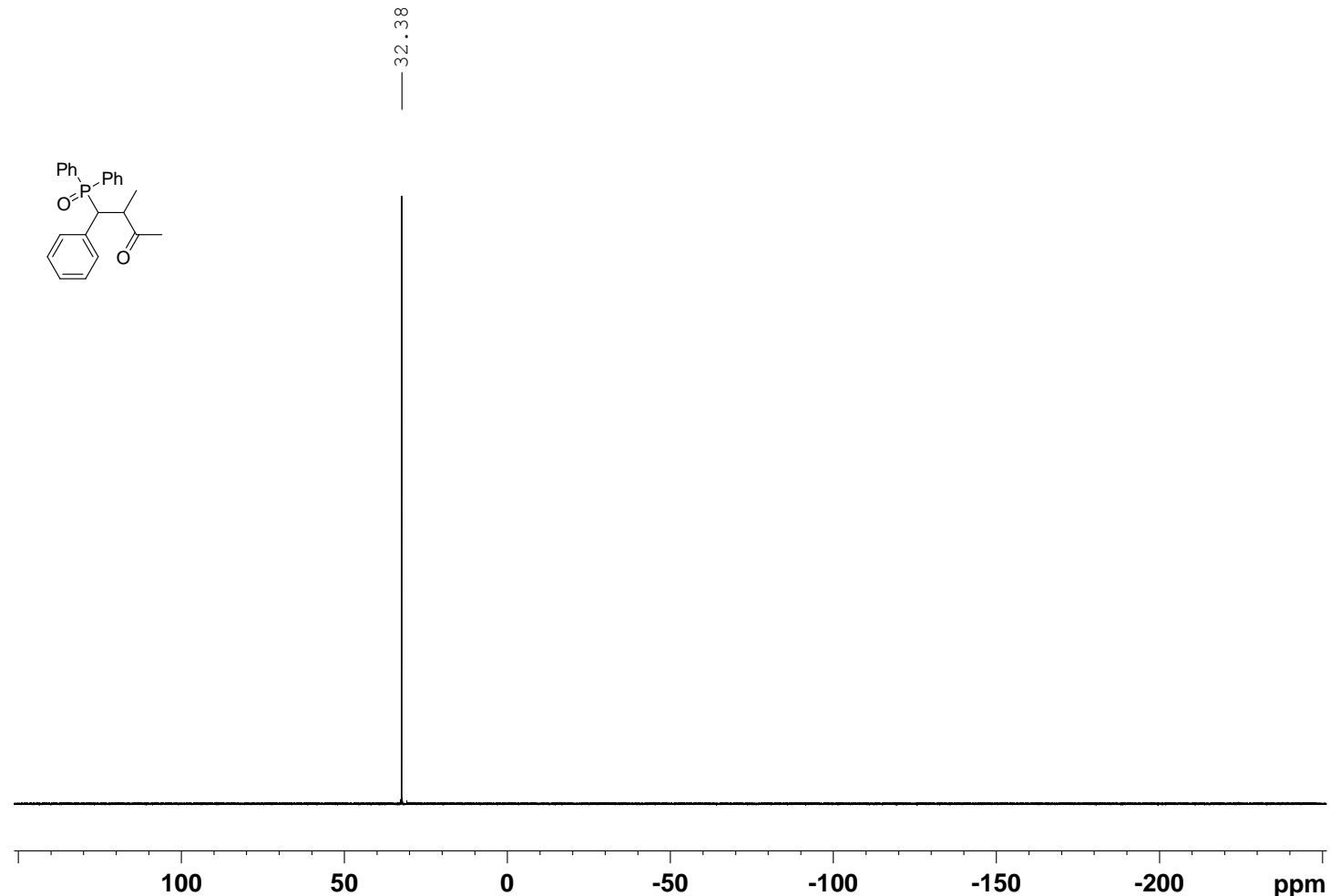
${ }^{1} \mathrm{H}$ NMR $\left(\mathrm{CDCl}_{3}, 500 \mathrm{MHz}\right)$ of 3ac:

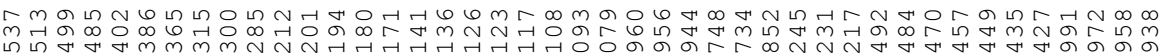

-

$=0$
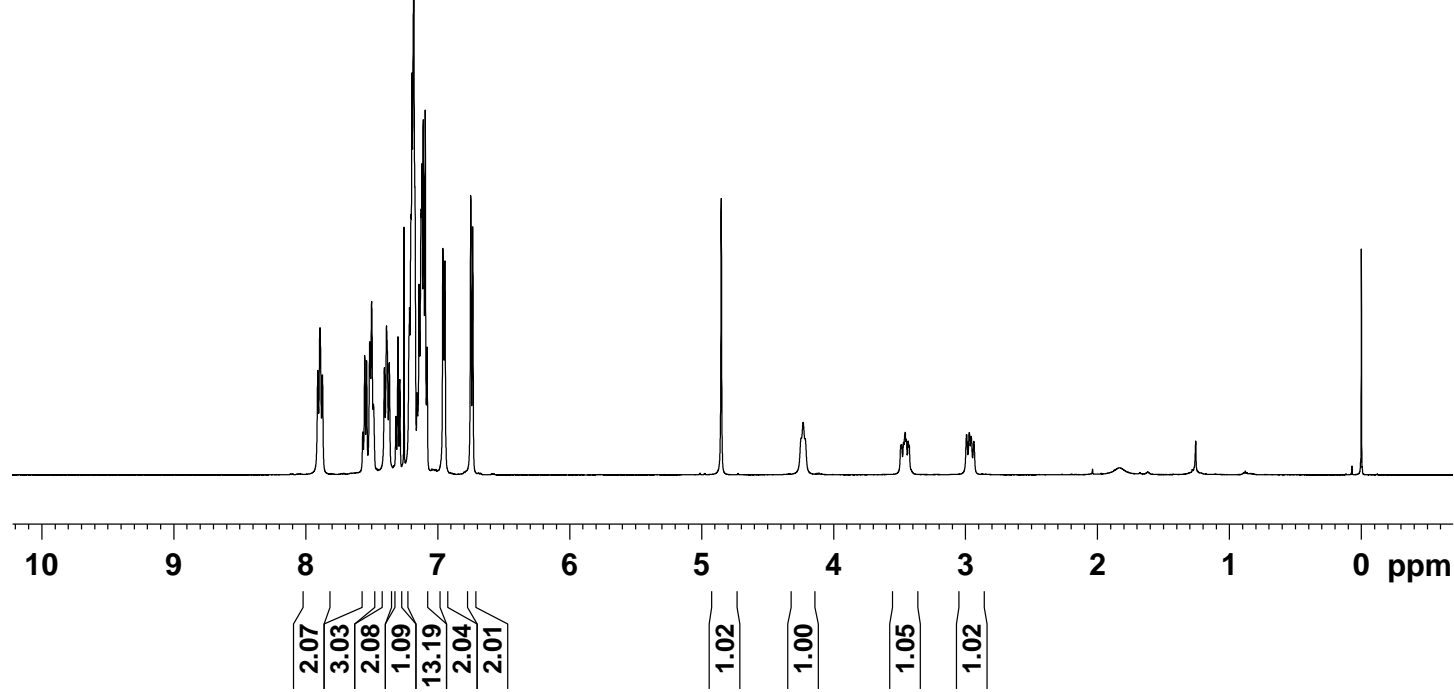

${ }^{13} \mathrm{C} \mathrm{NMR}\left(\mathrm{CDCl}_{3}, 125 \mathrm{MHz}\right)$ of 3ac:
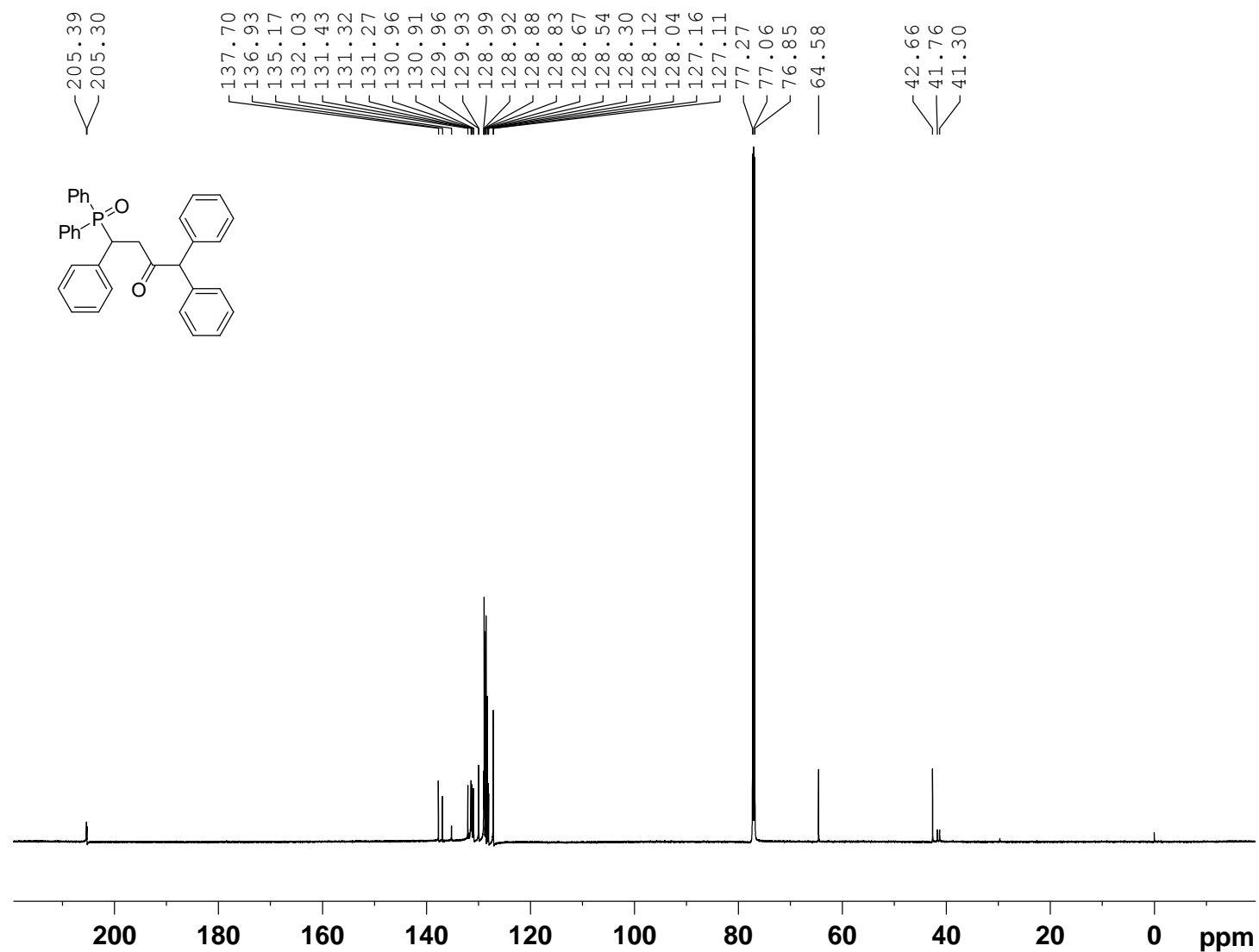
${ }^{31} \mathrm{P}$ NMR $\left(\mathrm{CDCl}_{3}, 202 \mathrm{MHz}\right)$ of 3ac:
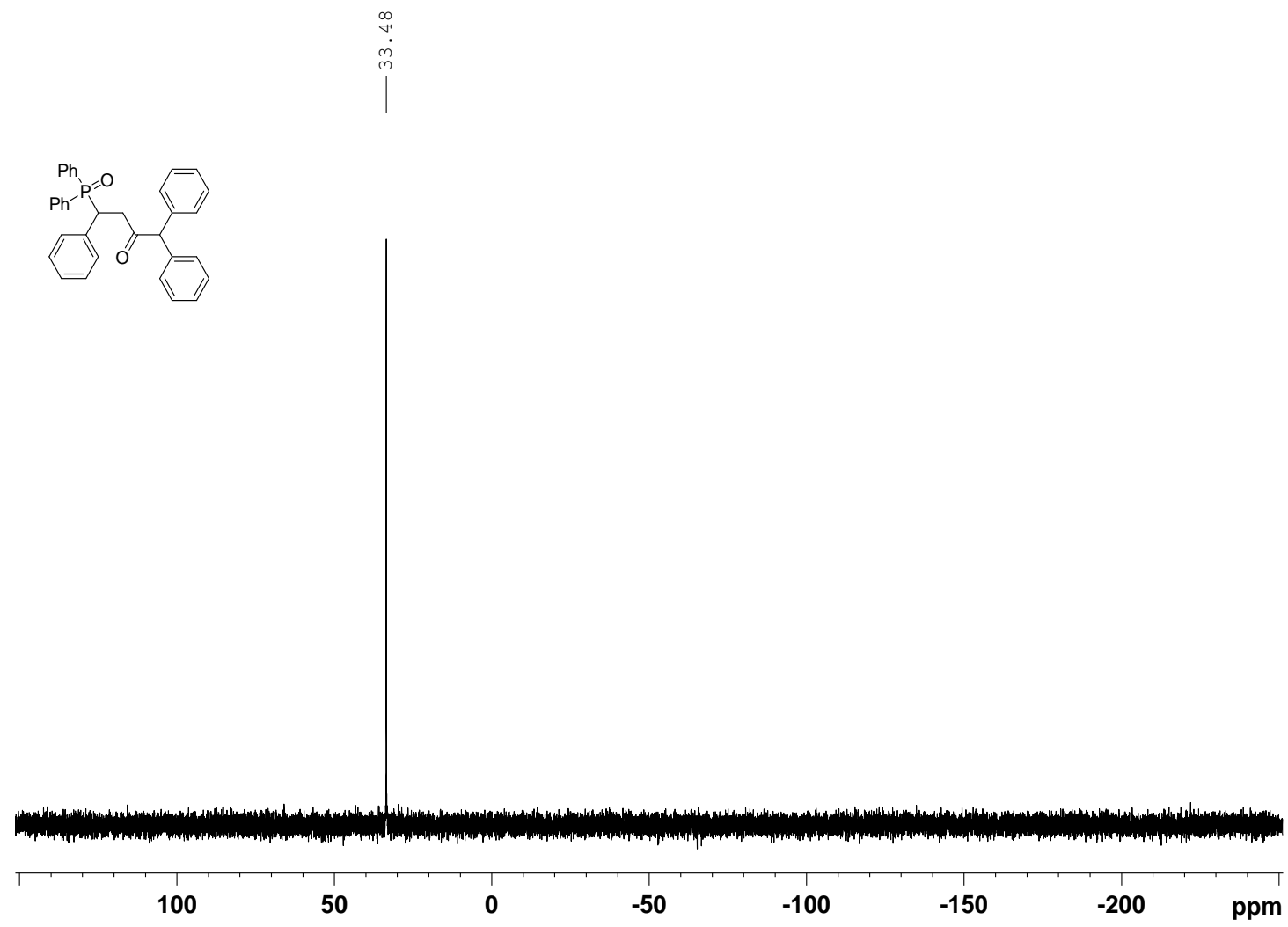

${ }^{1} \mathrm{H} \mathrm{NMR}\left(\mathrm{CDCl}_{3}, 500 \mathrm{MHz}\right)$ of $\mathbf{3 a d}$ :

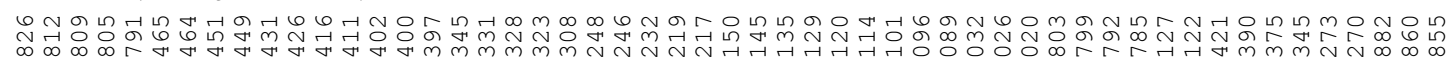

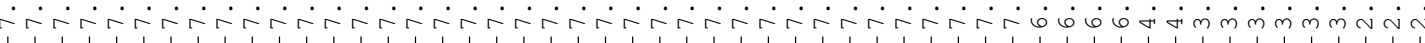

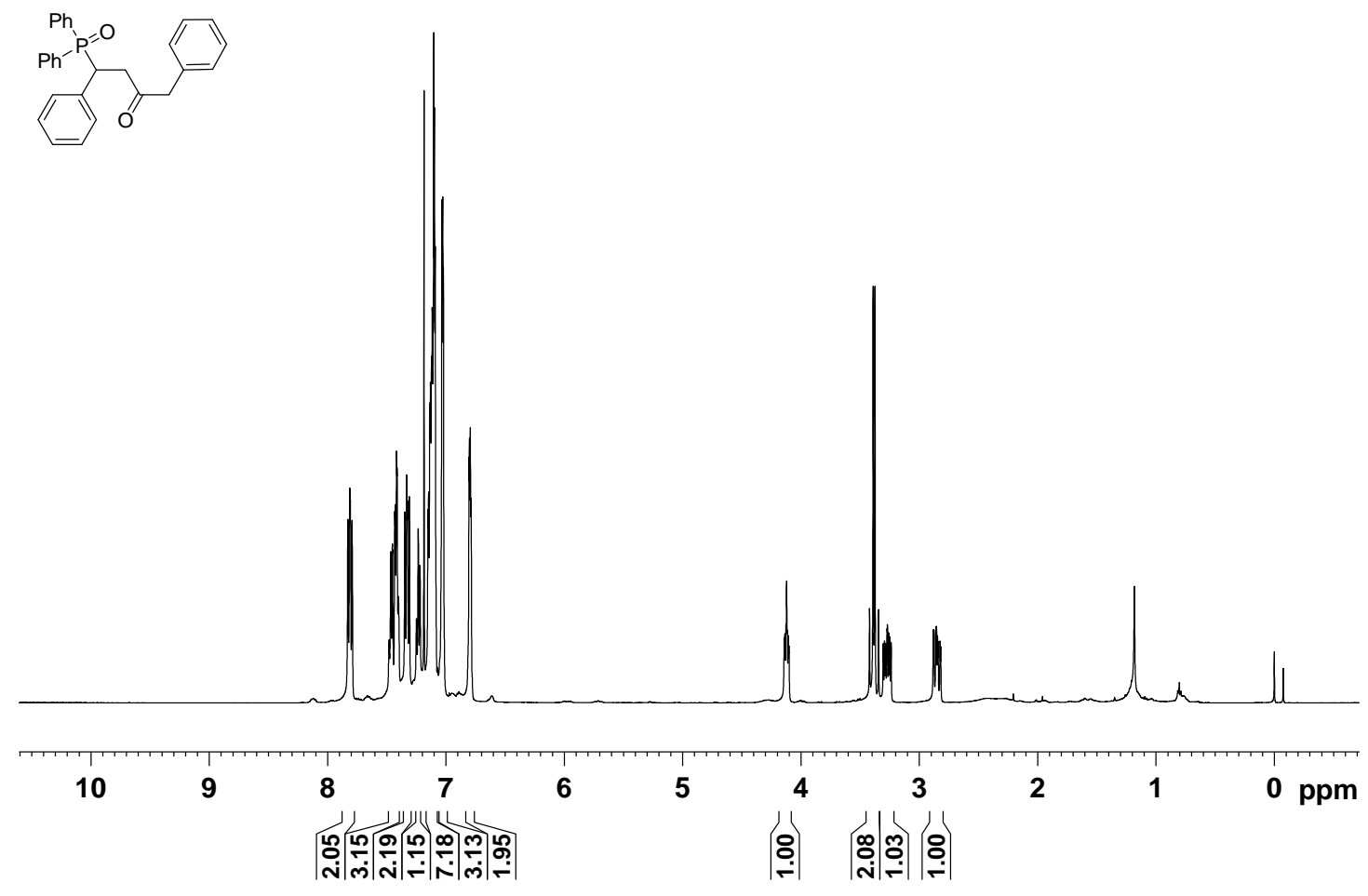


${ }^{13} \mathrm{C}$ NMR $\left(\mathrm{CDCl}_{3}, 125 \mathrm{MHz}\right)$ of $\mathbf{3 a d}$ :
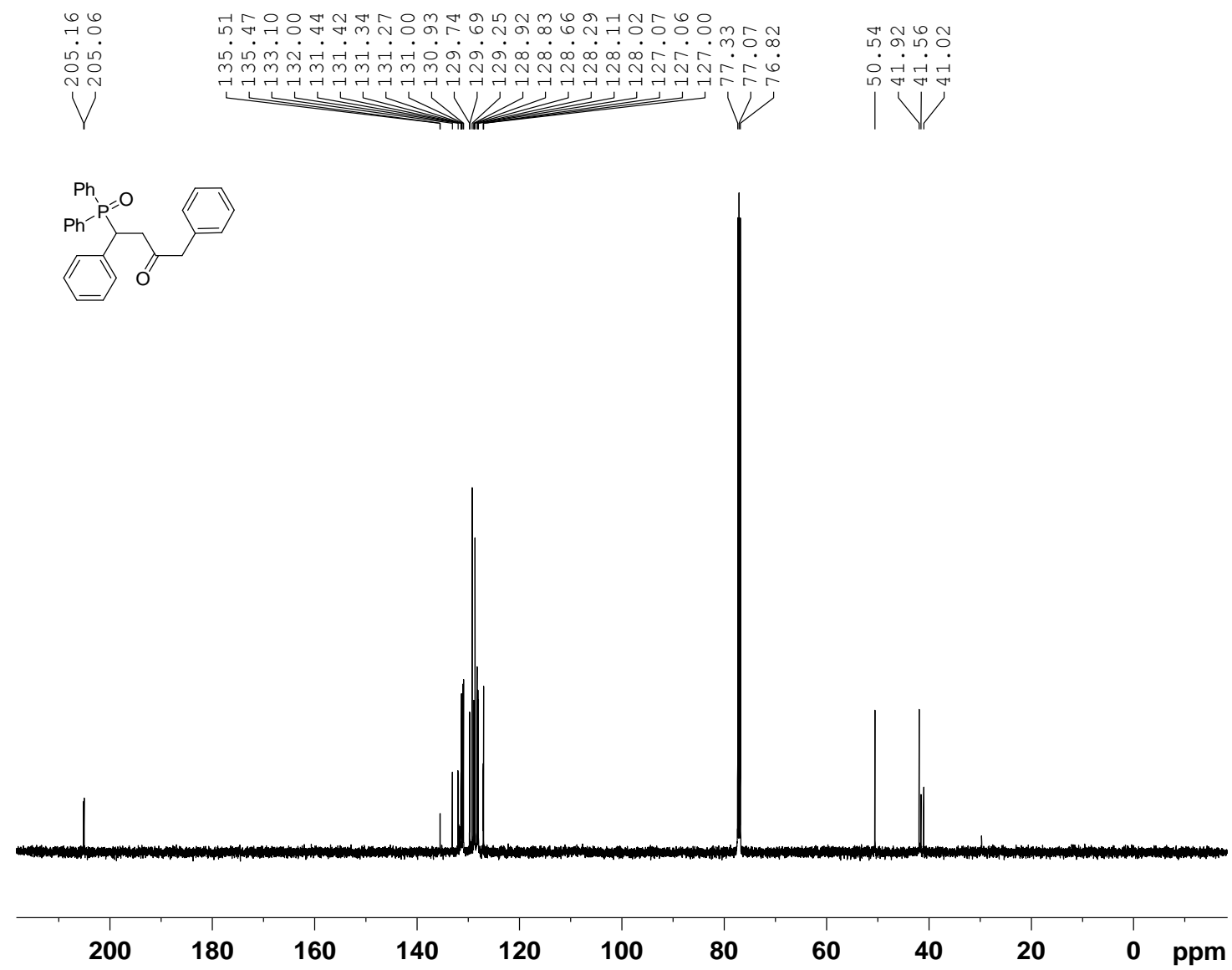

${ }^{31} \mathrm{P}$ NMR $\left(\mathrm{CDCl}_{3}, 202 \mathrm{MHz}\right)$ of 3ad:
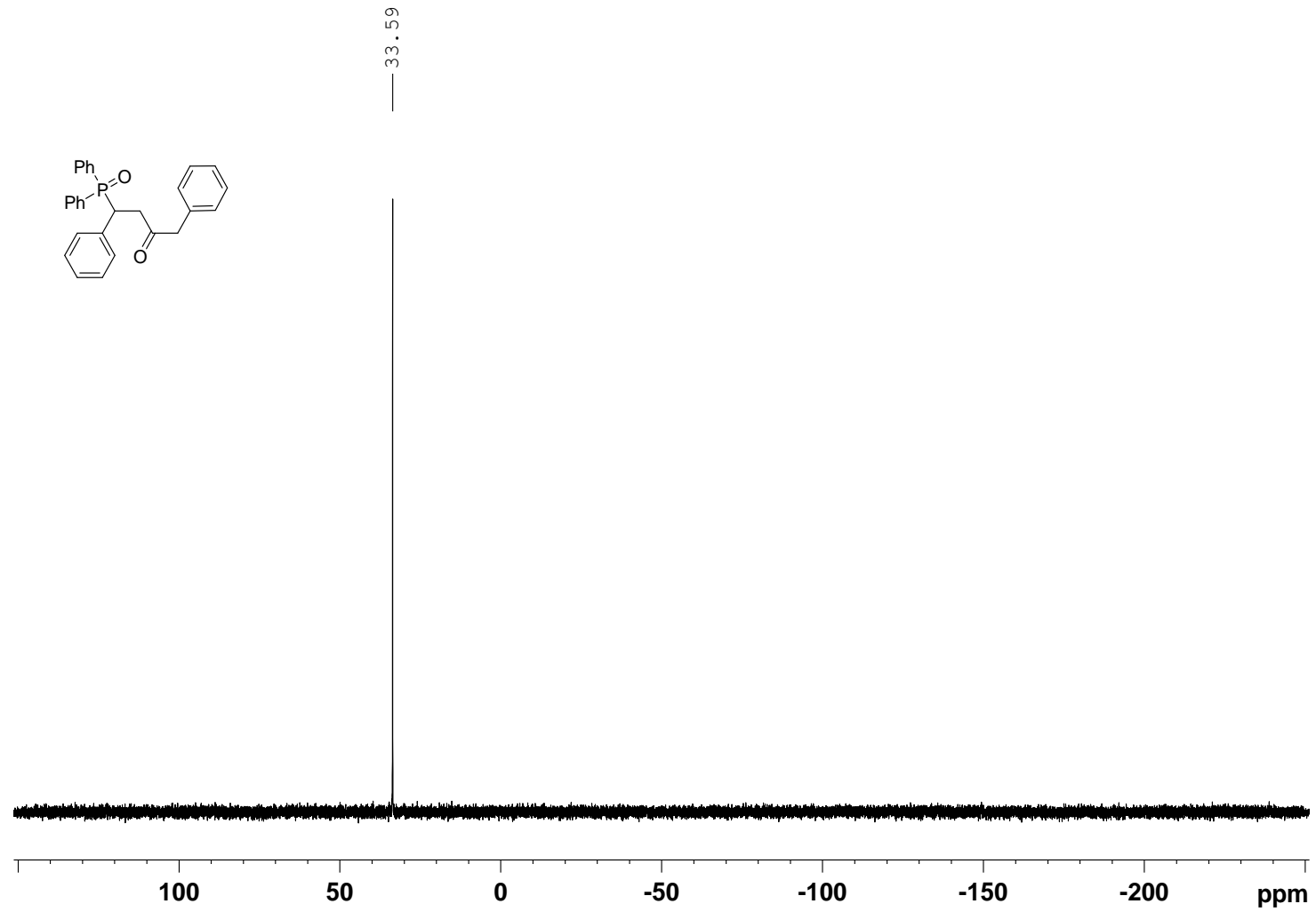
${ }^{1} \mathrm{H} \mathrm{NMR}\left(\mathrm{CDCl}_{3}, 500 \mathrm{MHz}\right)$ of $\mathbf{3 a e}:$

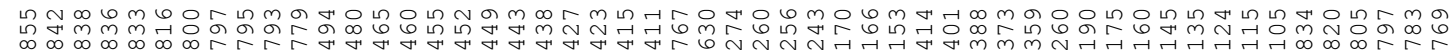

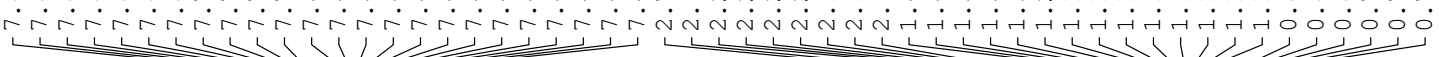
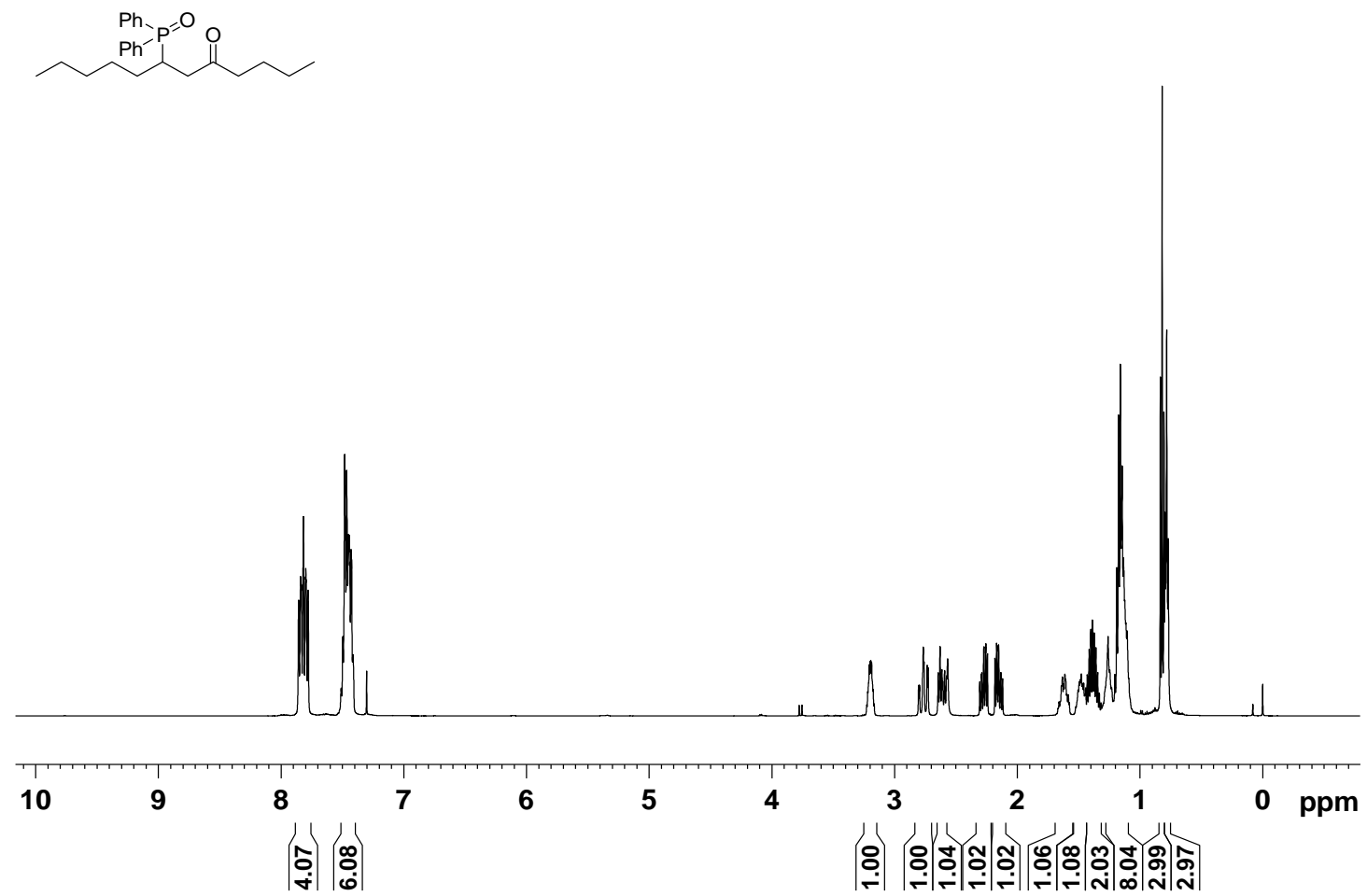

${ }^{13} \mathrm{C}$ NMR $\left(\mathrm{CDCl}_{3}, 125 \mathrm{MHz}\right)$ of $\mathbf{3 a e}:$
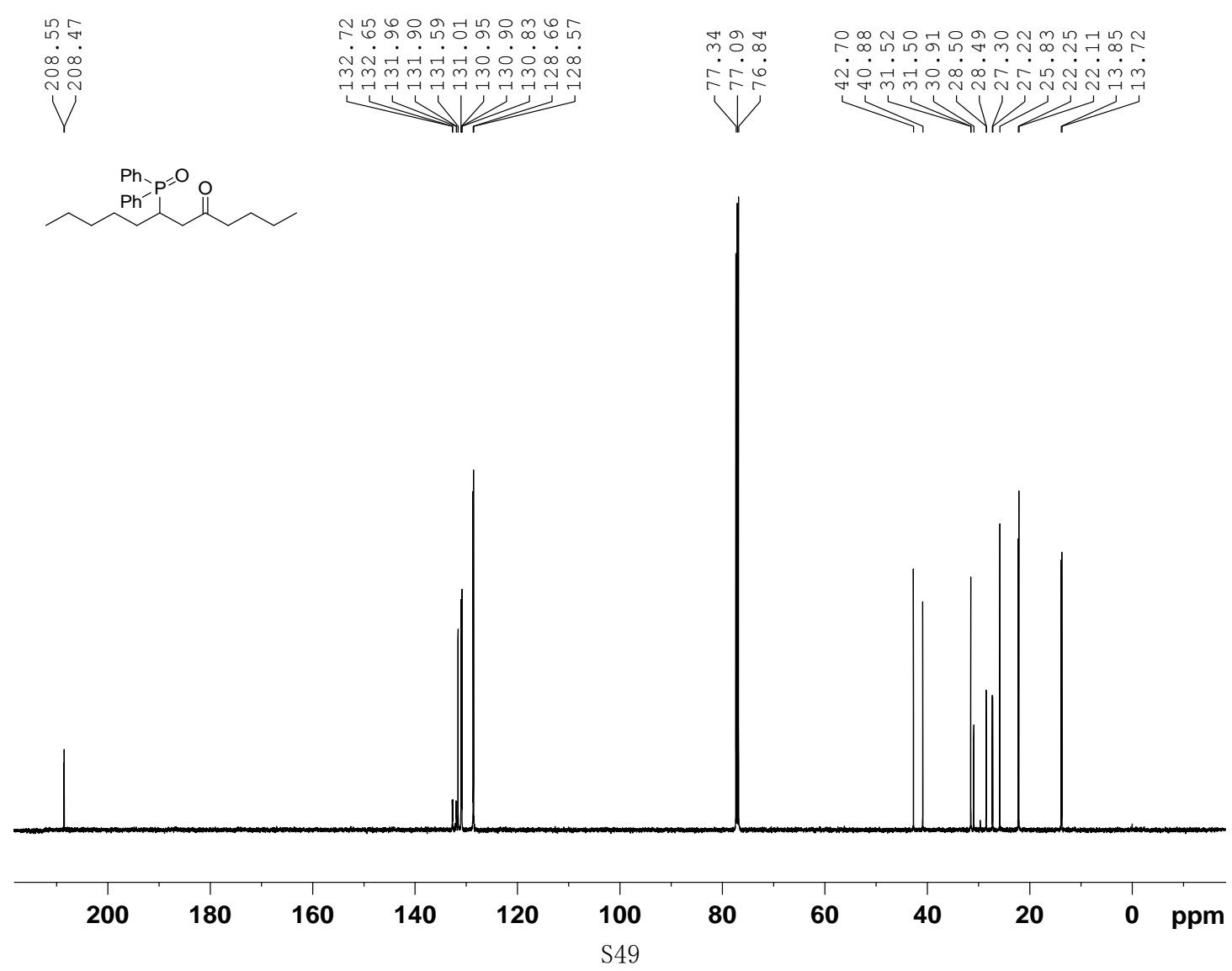
${ }^{31} \mathrm{P}$ NMR $\left(\mathrm{CDCl}_{3}, 202 \mathrm{MHz}\right)$ of 3ae:
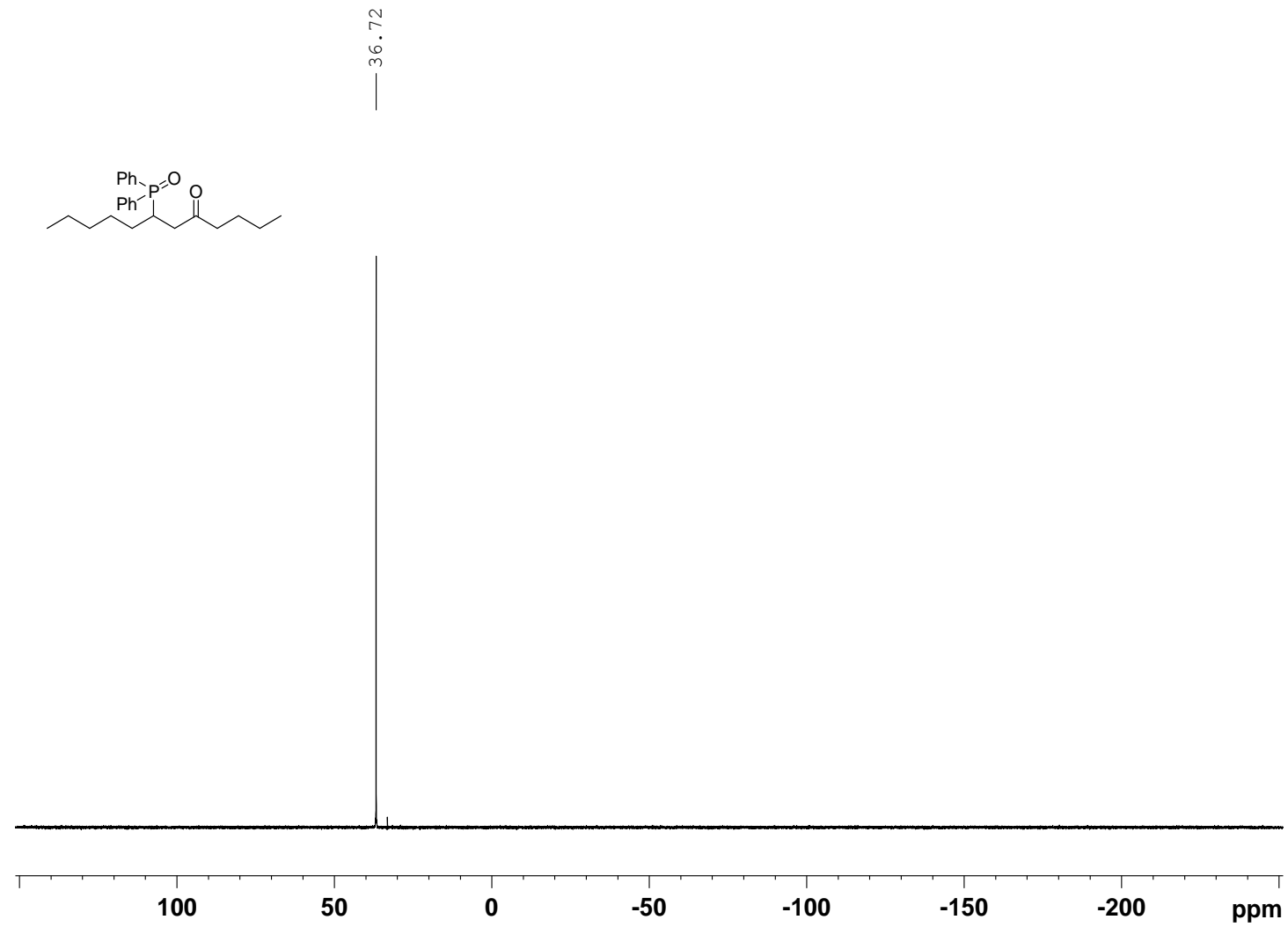

${ }^{1} \mathrm{H} \mathrm{NMR}\left(\mathrm{CDCl}_{3}, 500 \mathrm{MHz}\right)$ of $\mathbf{3 a f}:$

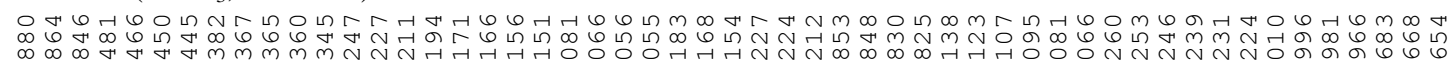
ninn (1)
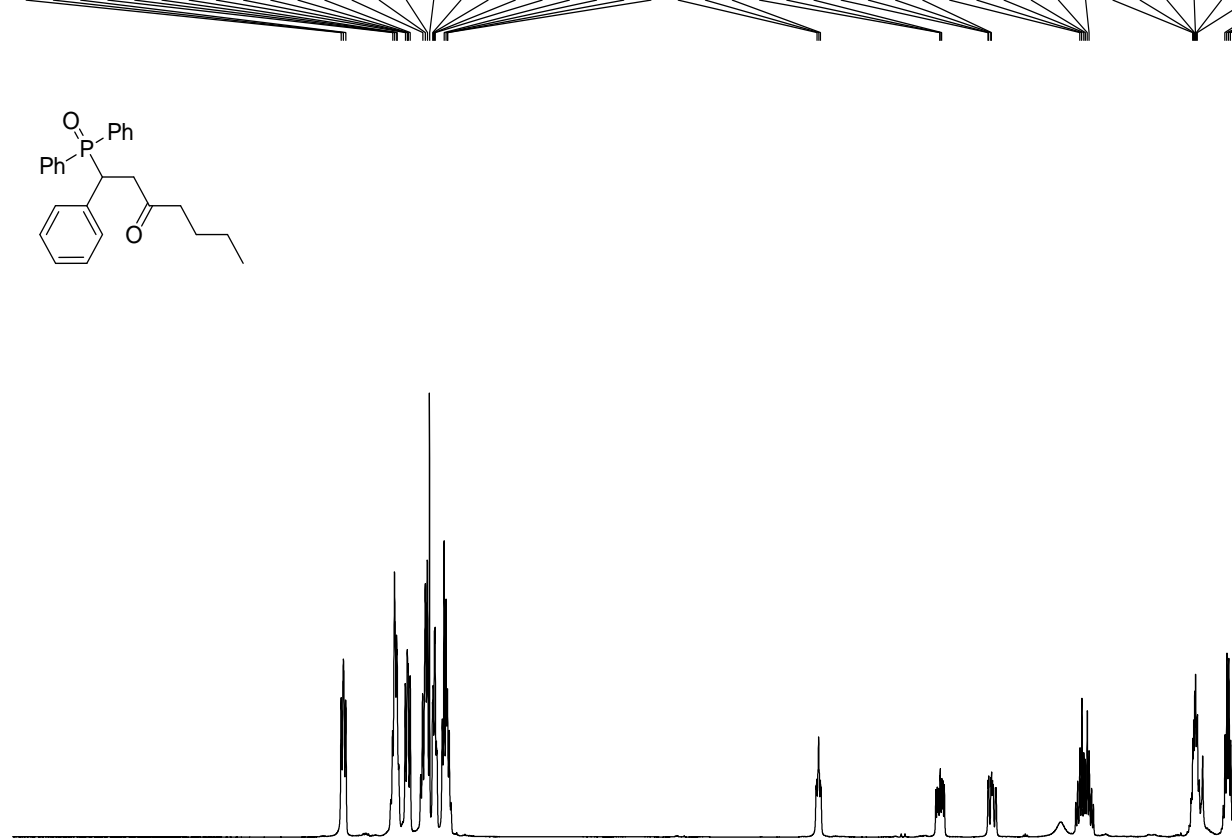
${ }^{13} \mathrm{C}$ NMR $\left(\mathrm{CDCl}_{3}, 125 \mathrm{MHz}\right)$ of 3af:
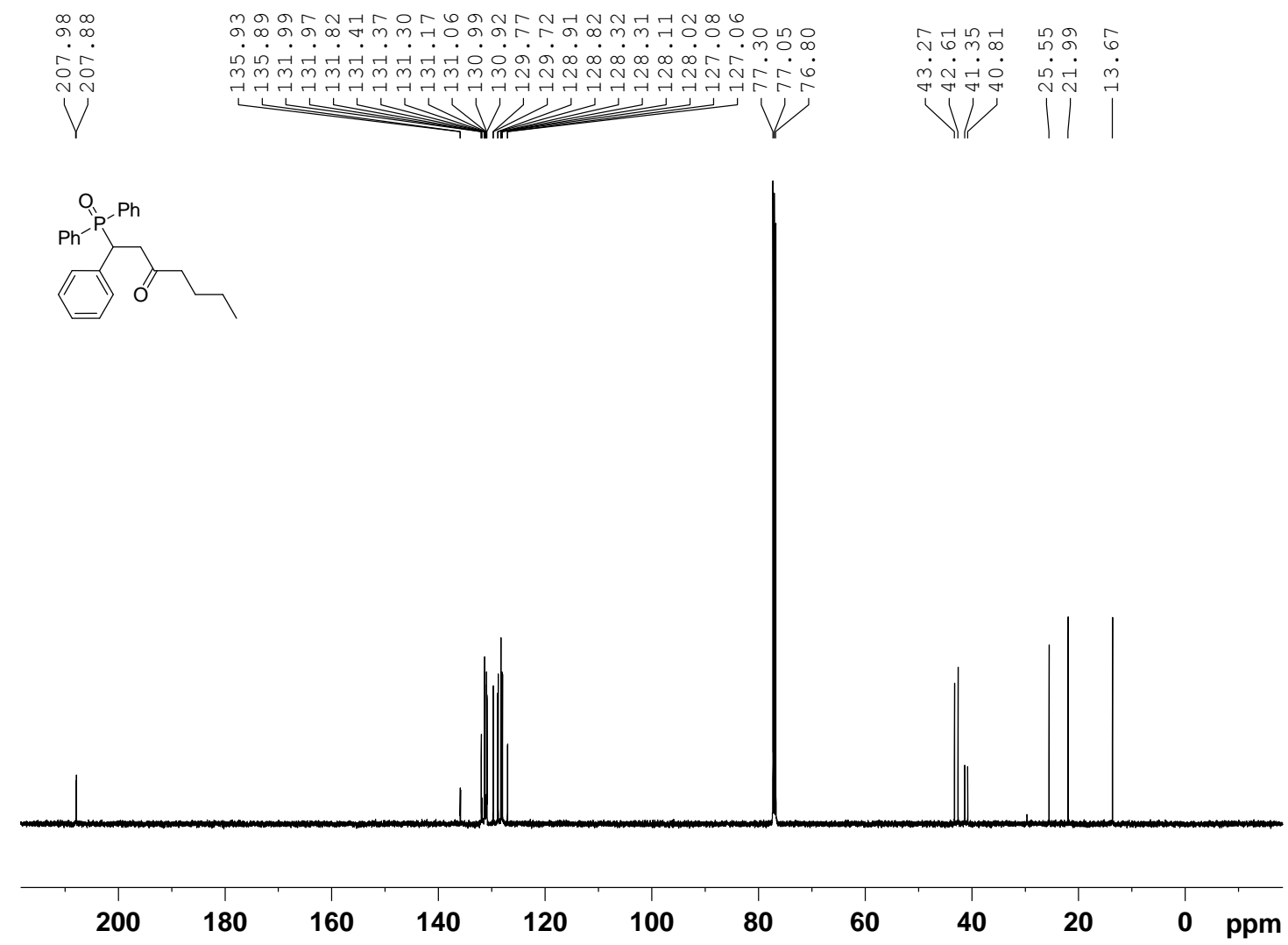

${ }^{31} \mathrm{P} \mathrm{NMR}\left(\mathrm{CDCl}_{3}, 202 \mathrm{MHz}\right)$ of 3af:
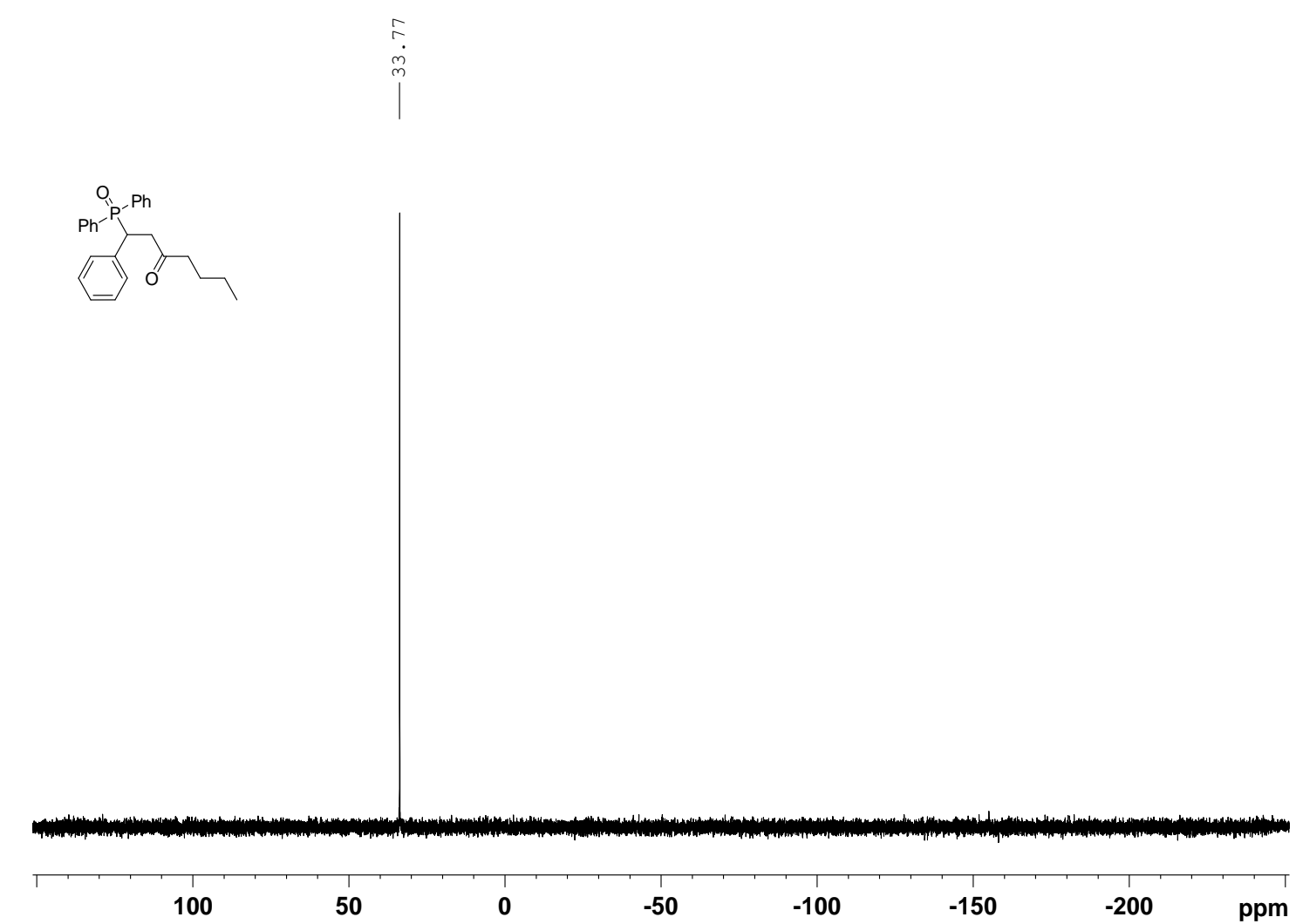
${ }^{1} \mathrm{H}$ NMR $\left(\mathrm{CDCl}_{3}, 500 \mathrm{MHz}\right)$ of $\mathbf{3 a g}$ :

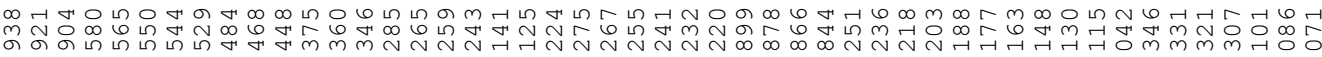
bititisitit
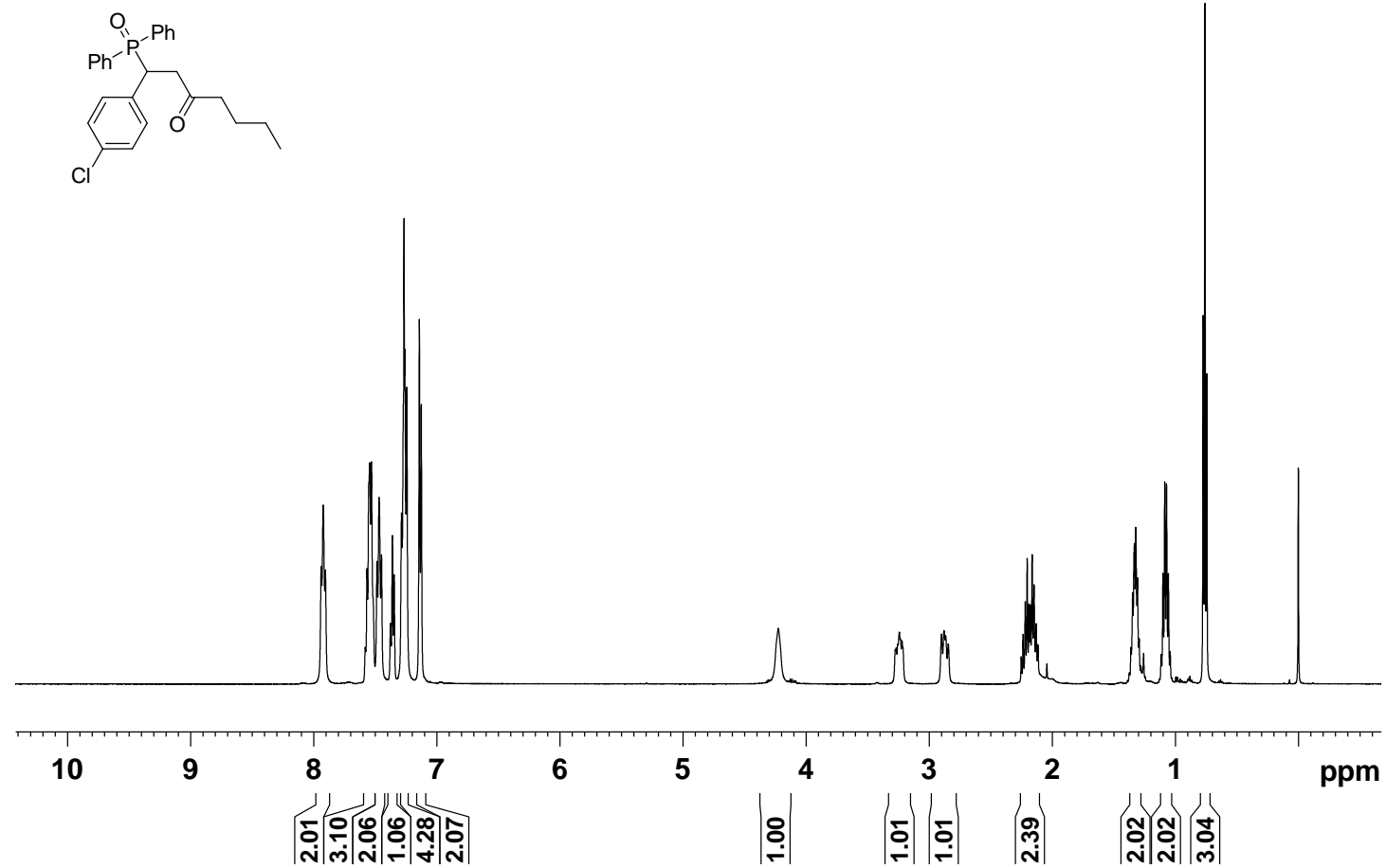

${ }^{13} \mathrm{C} \mathrm{NMR}\left(\mathrm{CDCl}_{3}, 125 \mathrm{MHz}\right)$ of 3ag:
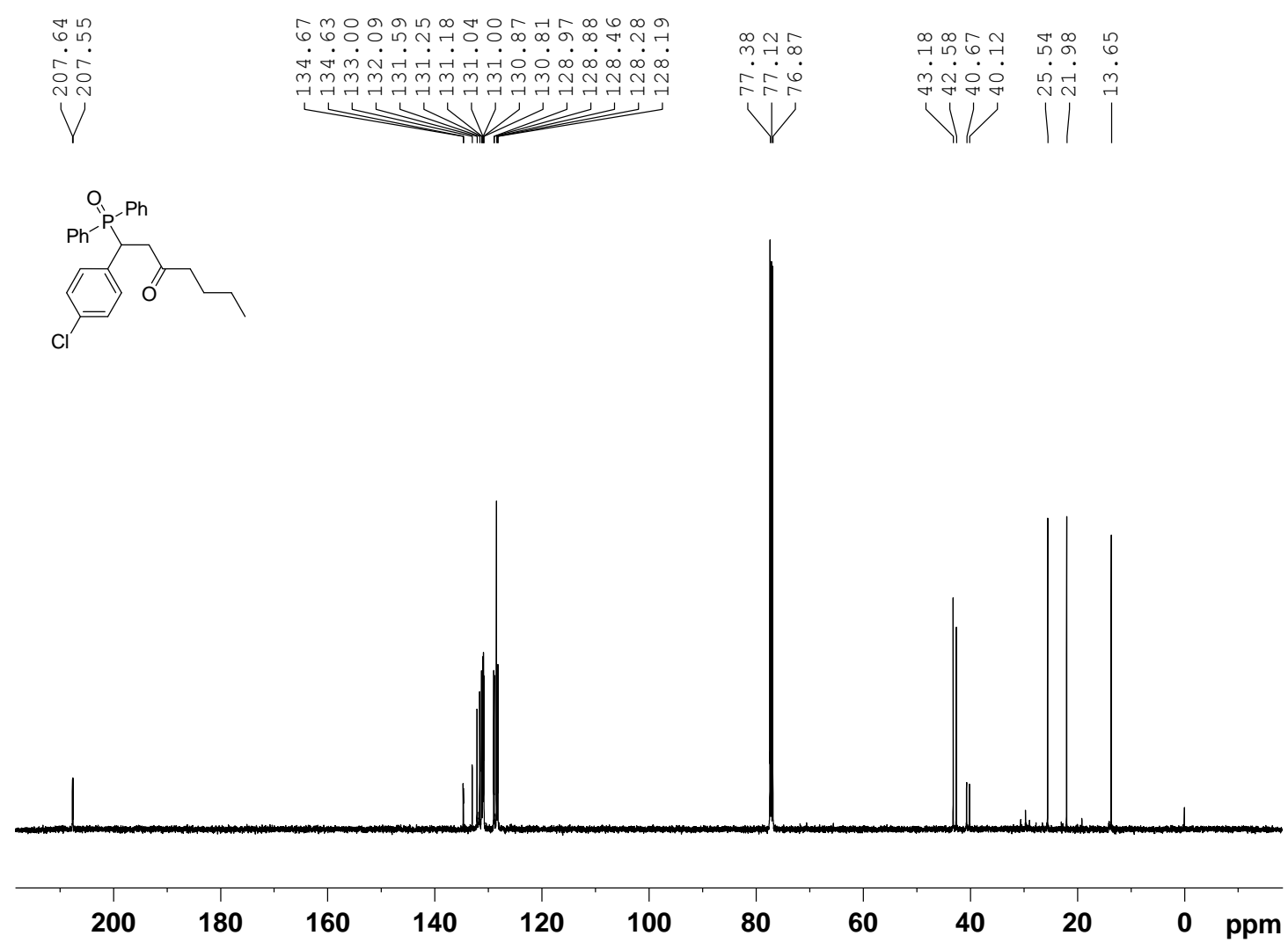
${ }^{31} \mathrm{P}$ NMR $\left(\mathrm{CDCl}_{3}, 202 \mathrm{MHz}\right)$ of 3ag:<smiles>CN=NC</smiles>

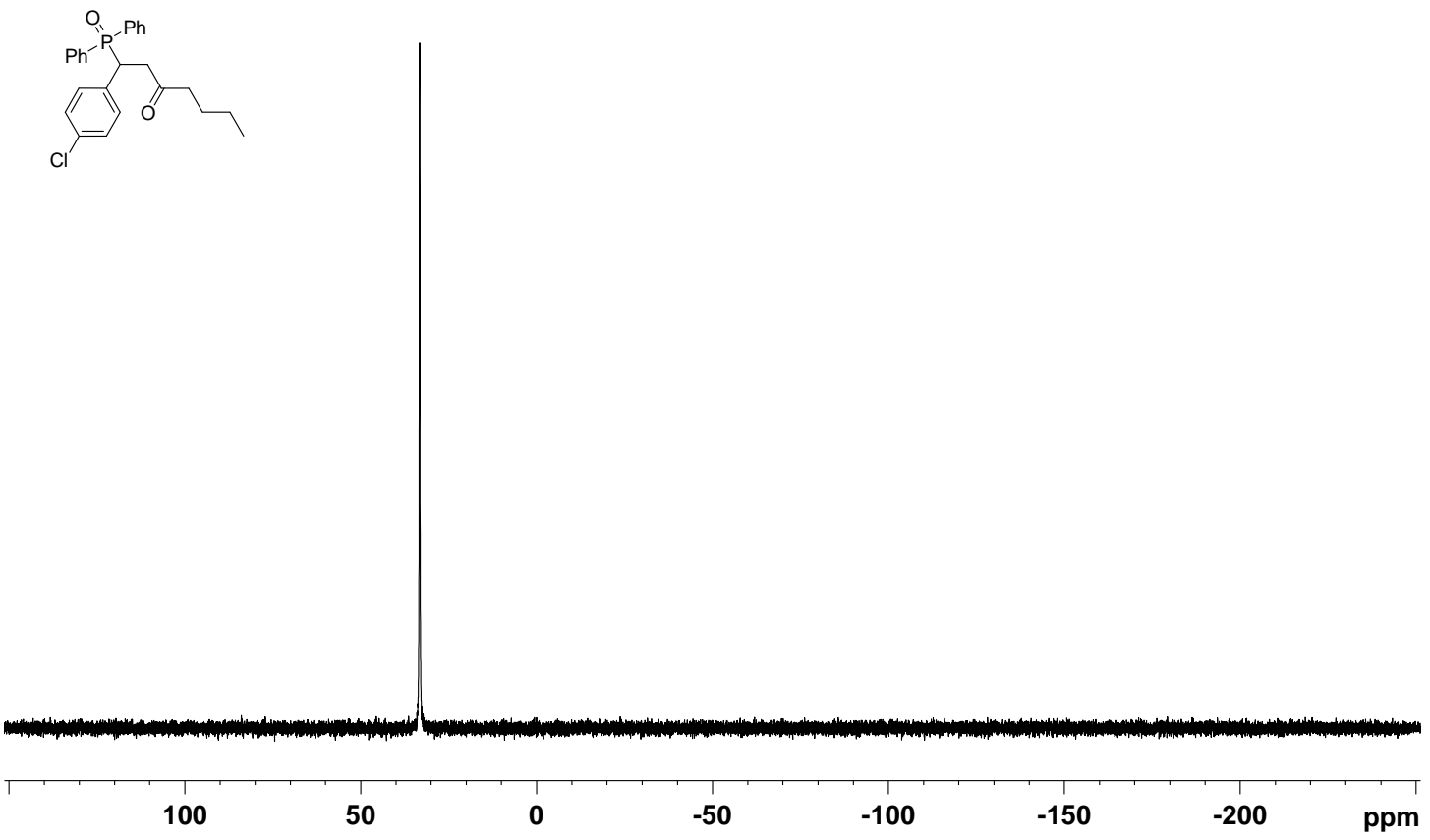

${ }^{1} \mathrm{H} \mathrm{NMR}\left(\mathrm{CDCl}_{3}, 500 \mathrm{MHz}\right)$ of $\mathbf{3 a h}:$

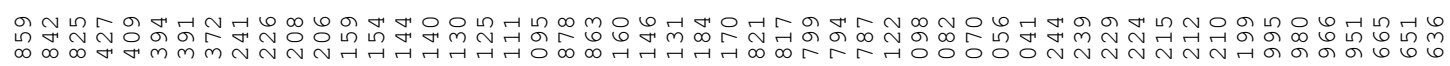

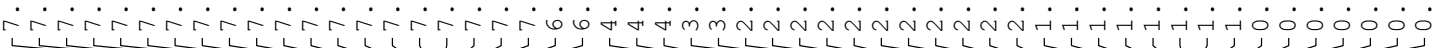
$\underbrace{1}_{\text {111 }}$
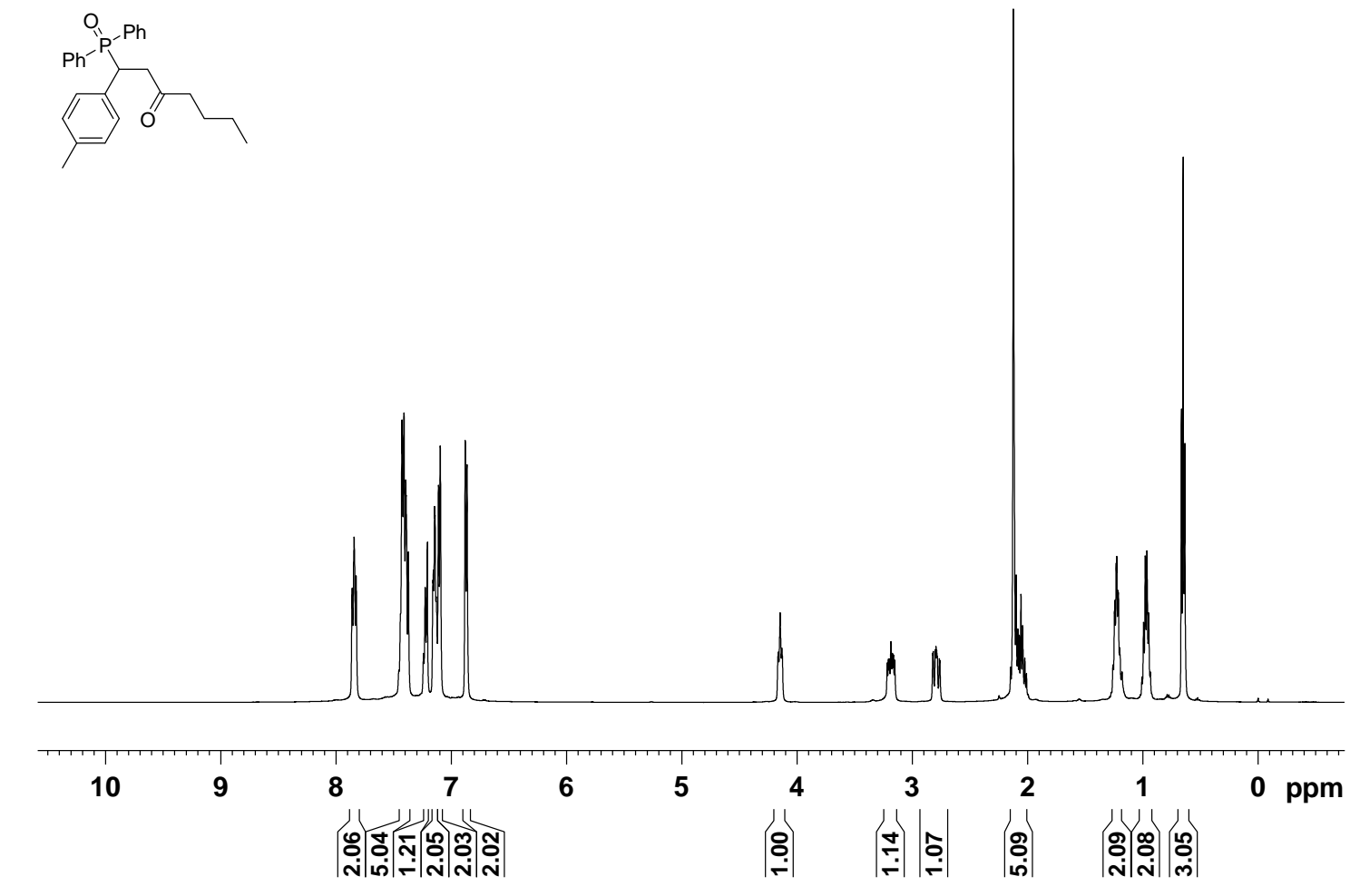
${ }^{13} \mathrm{C}$ NMR $\left(\mathrm{CDCl}_{3}, 125 \mathrm{MHz}\right)$ of $\mathbf{3 a h}$ :
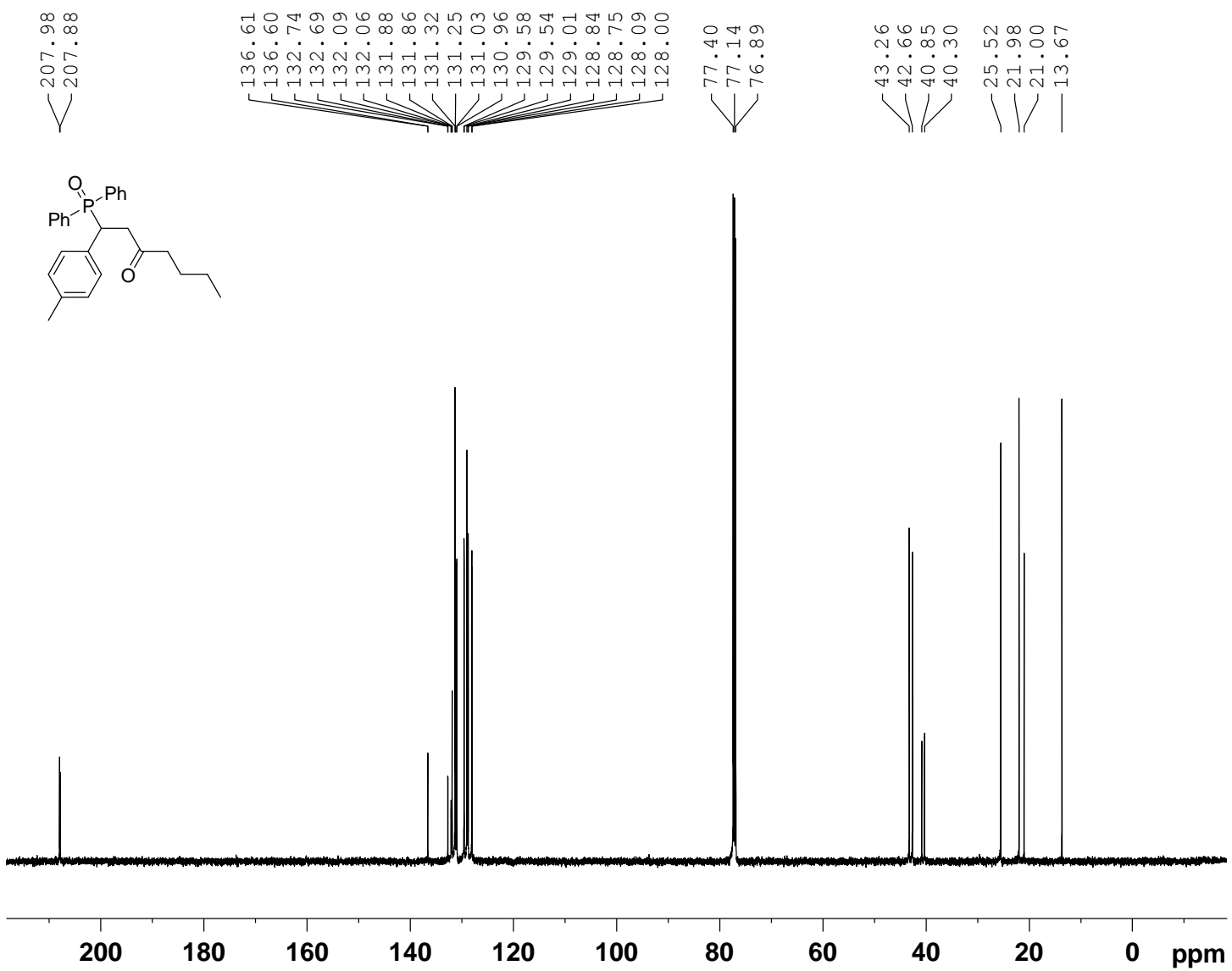

${ }^{31} \mathrm{P}$ NMR $\left(\mathrm{CDCl}_{3}, 202 \mathrm{MHz}\right)$ of $\mathbf{3 a h}$ :
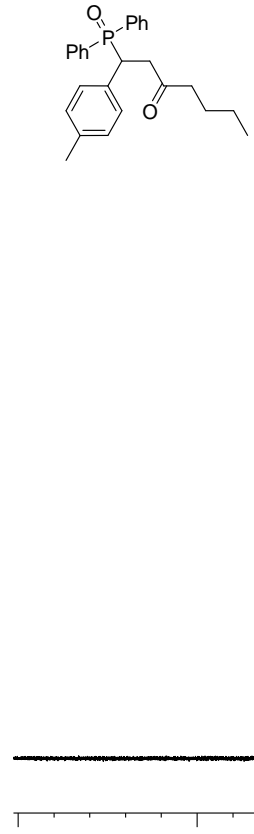

50

0

$-50$

$-100$

$-150$

$-200$

ppm 
${ }^{1} \mathrm{H} \mathrm{NMR}\left(\mathrm{CDCl}_{3}, 500 \mathrm{MHz}\right)$ of 3ai:

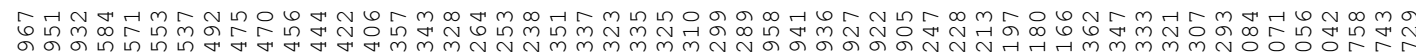
-

(n)
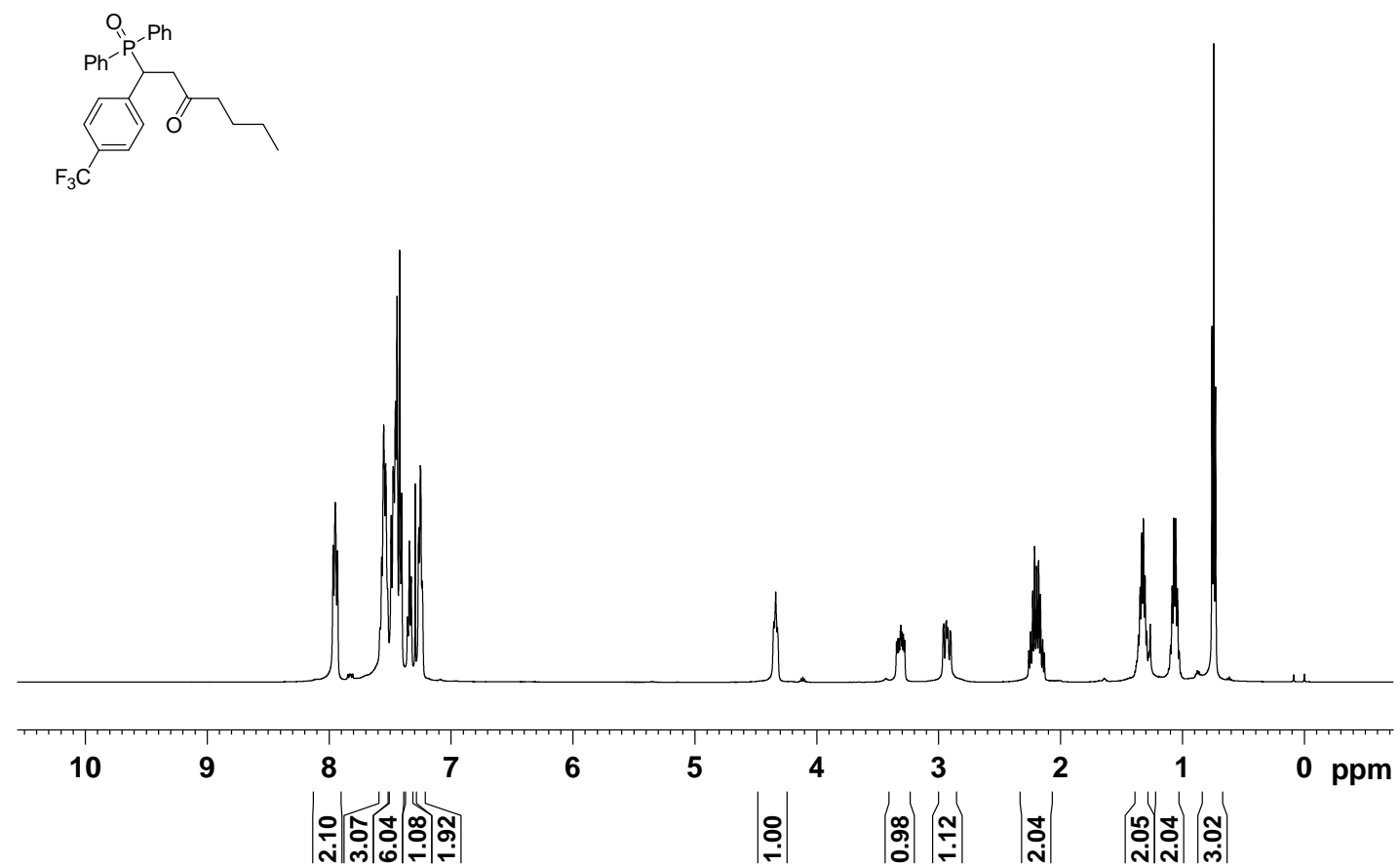

${ }^{13} \mathrm{C} \mathrm{NMR}\left(\mathrm{CDCl}_{3}, 125 \mathrm{MHz}\right)$ of 3ai:
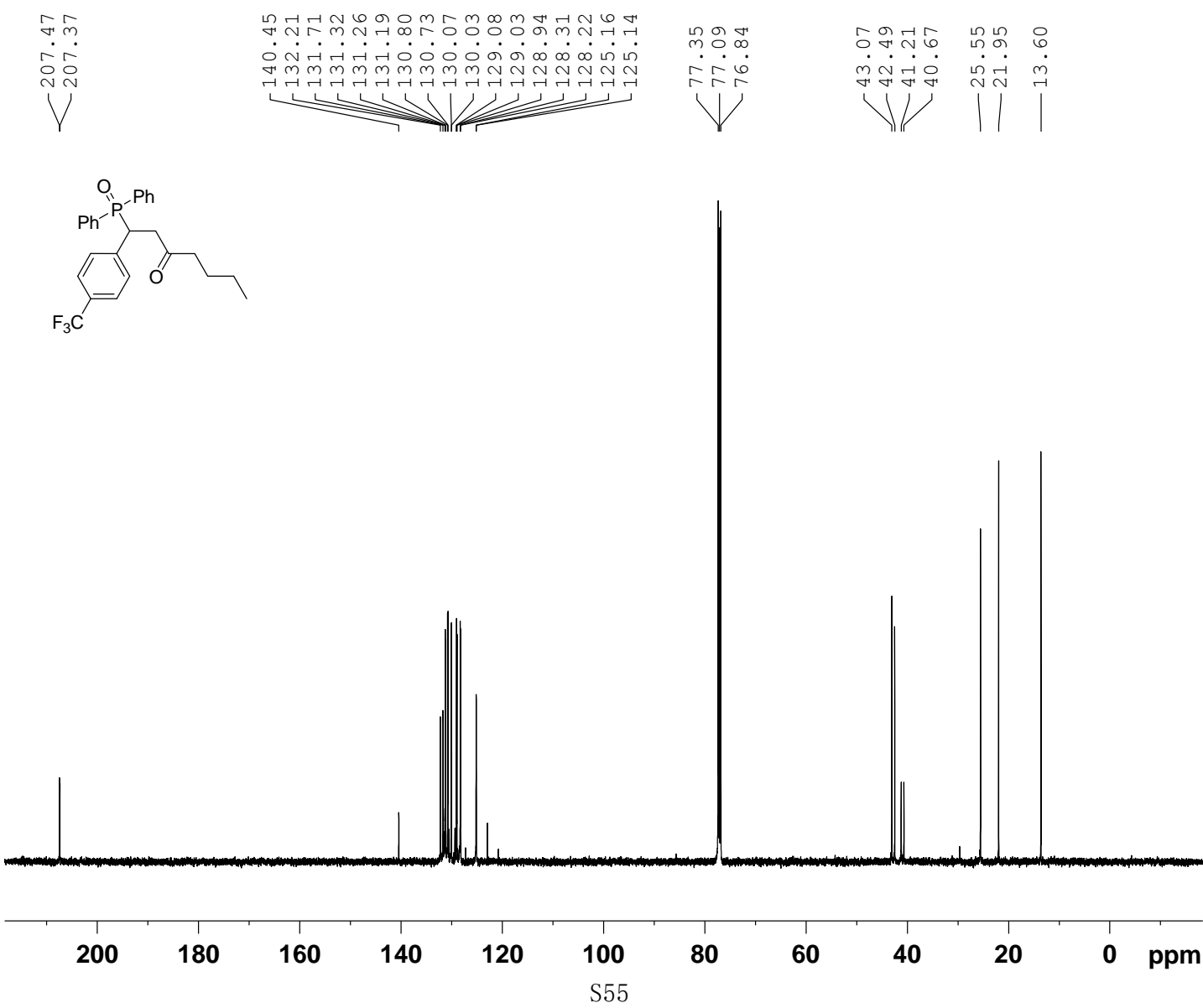
${ }^{31} \mathrm{P}$ NMR $\left(\mathrm{CDCl}_{3}, 202 \mathrm{MHz}\right)$ of 3ai:

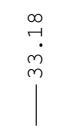

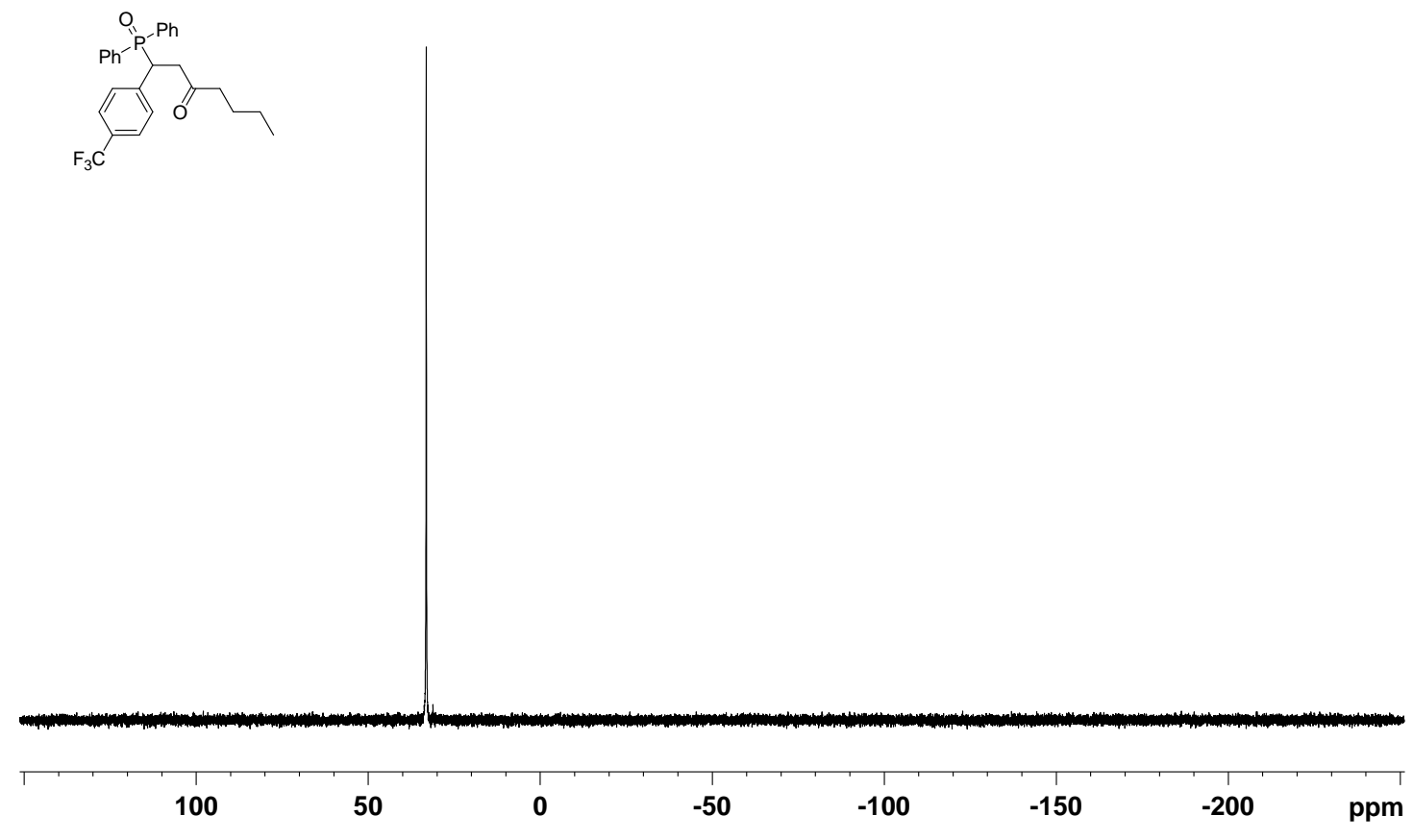

${ }^{1} \mathrm{H} \mathrm{NMR}\left(\mathrm{CDCl}_{3}, 500 \mathrm{MHz}\right)$ of $\mathbf{3 a j}:$

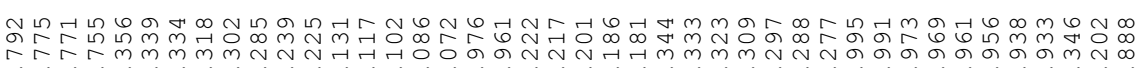

rrirrir

$\longrightarrow$
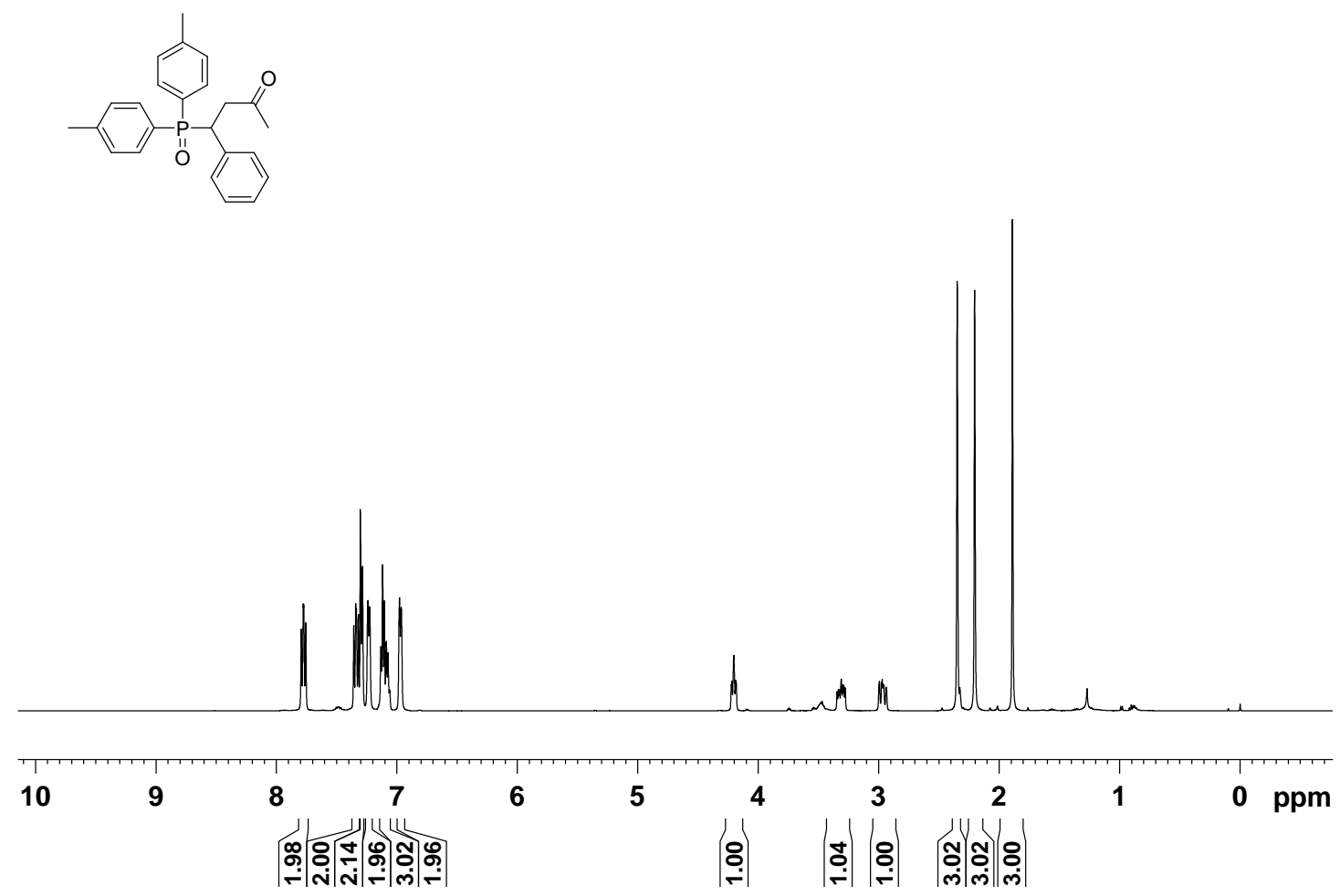
${ }^{13} \mathrm{C}$ NMR $\left(\mathrm{CDCl}_{3}, 125 \mathrm{MHz}\right)$ of $\mathbf{3 a j}:$
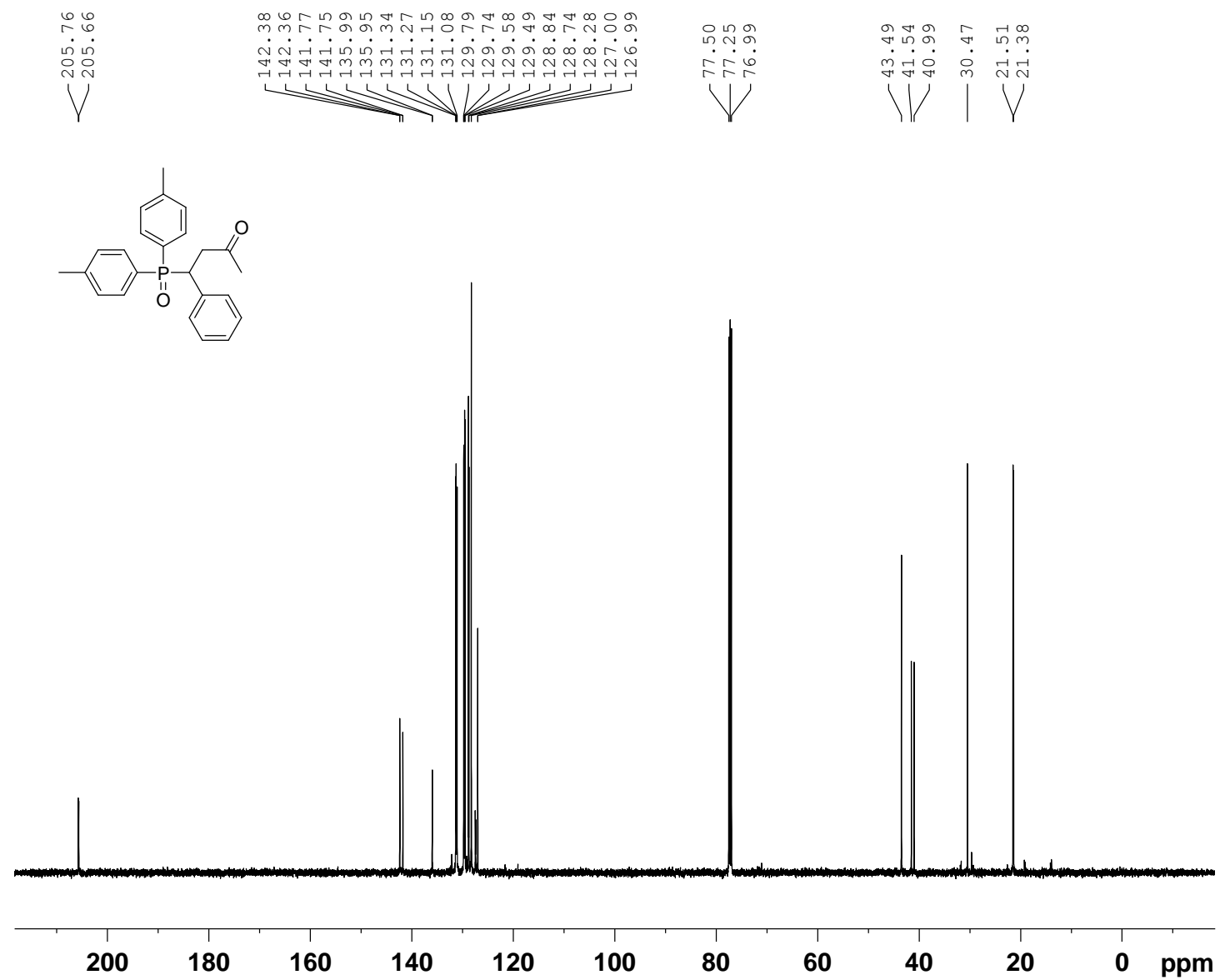

${ }^{31} \mathrm{P}$ NMR $\left(\mathrm{CDCl}_{3}, 202 \mathrm{MHz}\right)$ of 3aj:
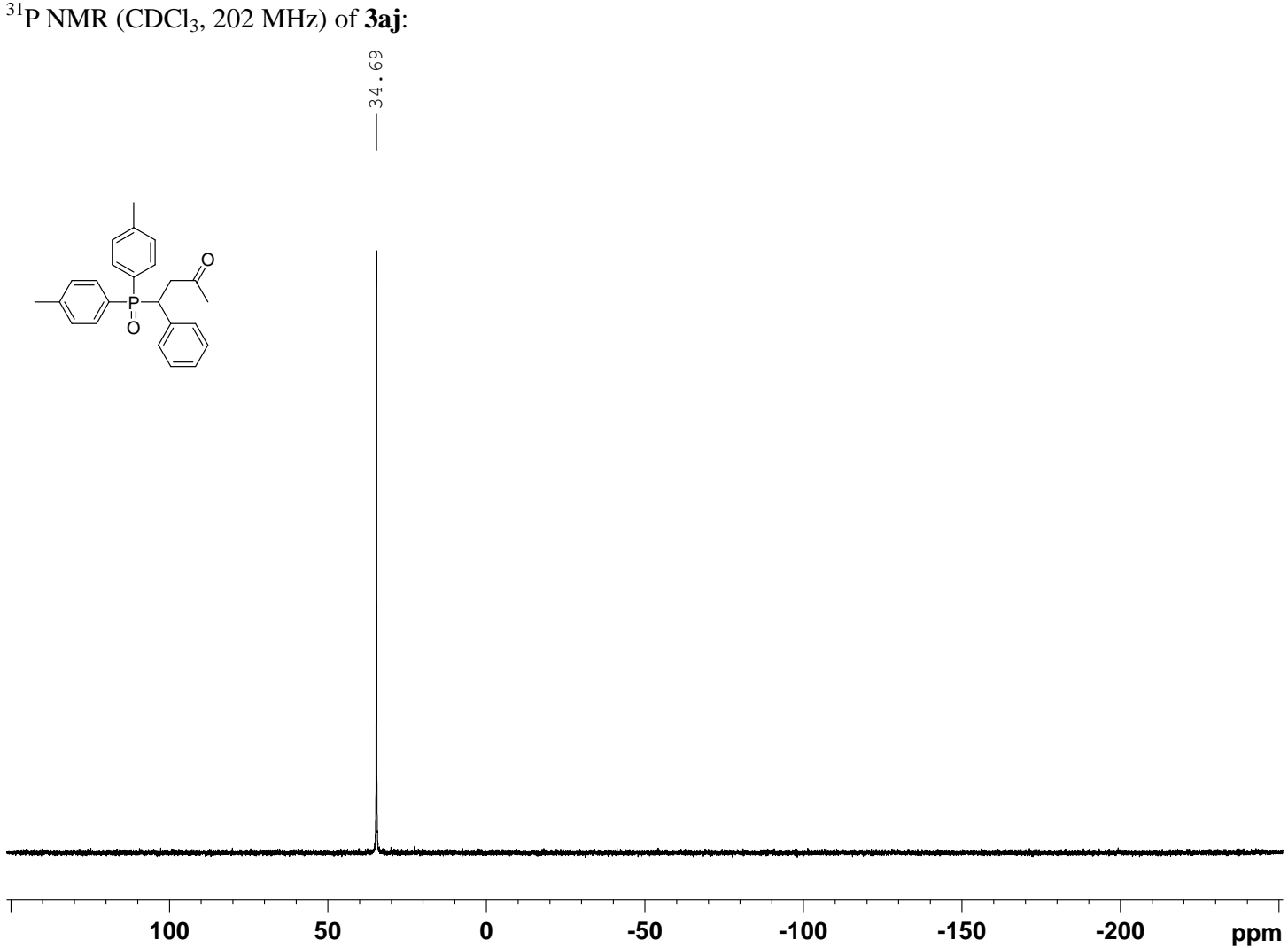
${ }^{1} \mathrm{H}$ NMR $\left(\mathrm{CDCl}_{3}, 500 \mathrm{MHz}\right)$ of $\mathbf{3 a k}$ :
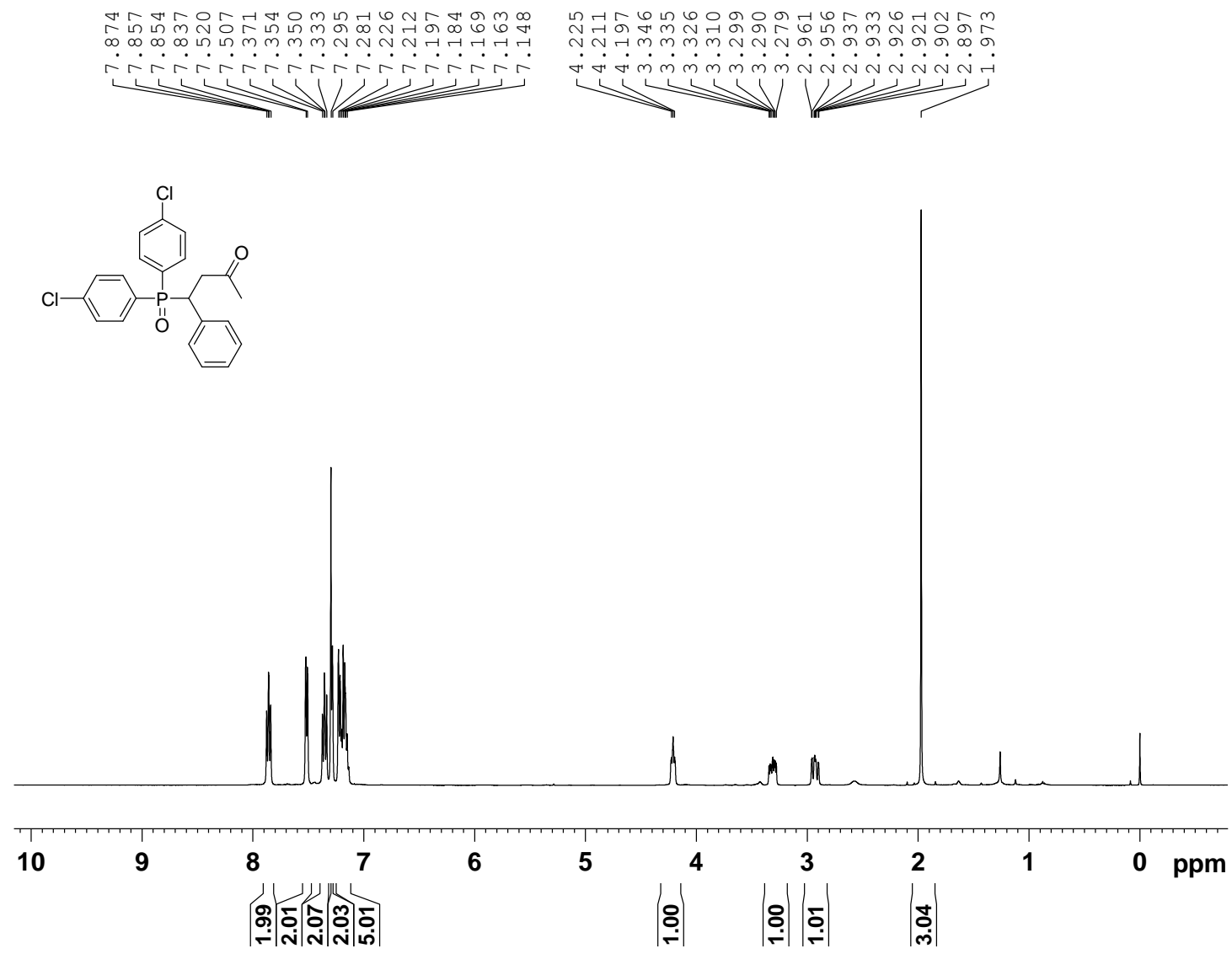

${ }^{13} \mathrm{C} \mathrm{NMR}\left(\mathrm{CDCl}_{3}, 125 \mathrm{MHz}\right)$ of 3ak:
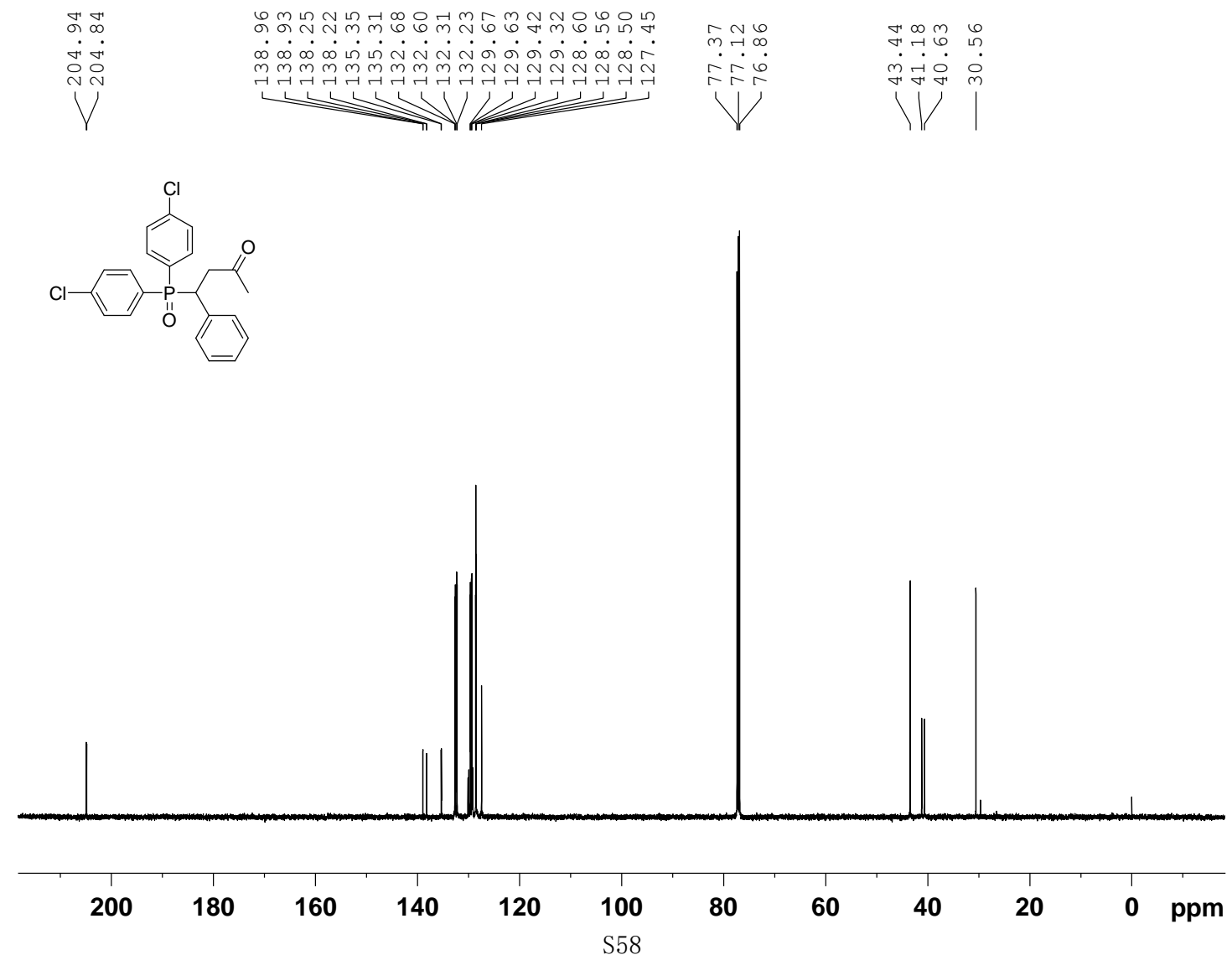
${ }^{31} \mathrm{P} \mathrm{NMR}\left(\mathrm{CDCl}_{3}, 202 \mathrm{MHz}\right)$ of 3ak:
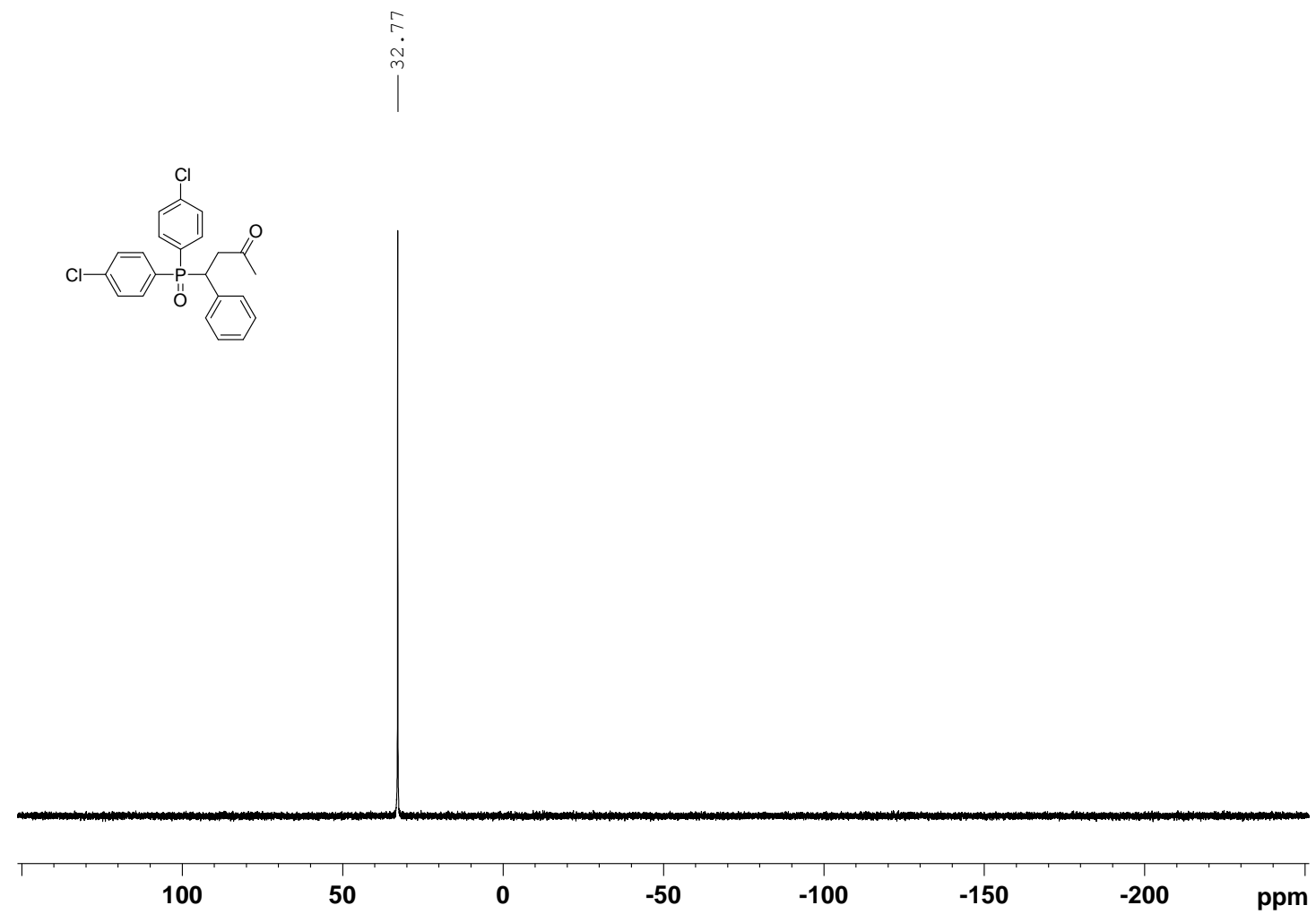

${ }^{1} \mathrm{H} \mathrm{NMR}\left(\mathrm{CDCl}_{3}, 500 \mathrm{MHz}\right)$ of $\mathbf{3 a l}:$

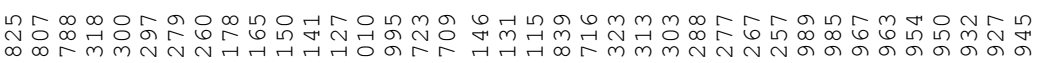

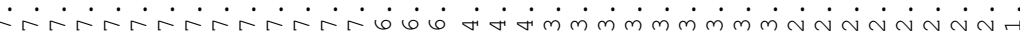

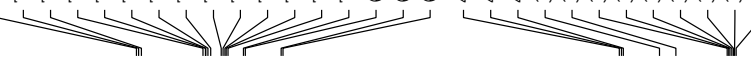
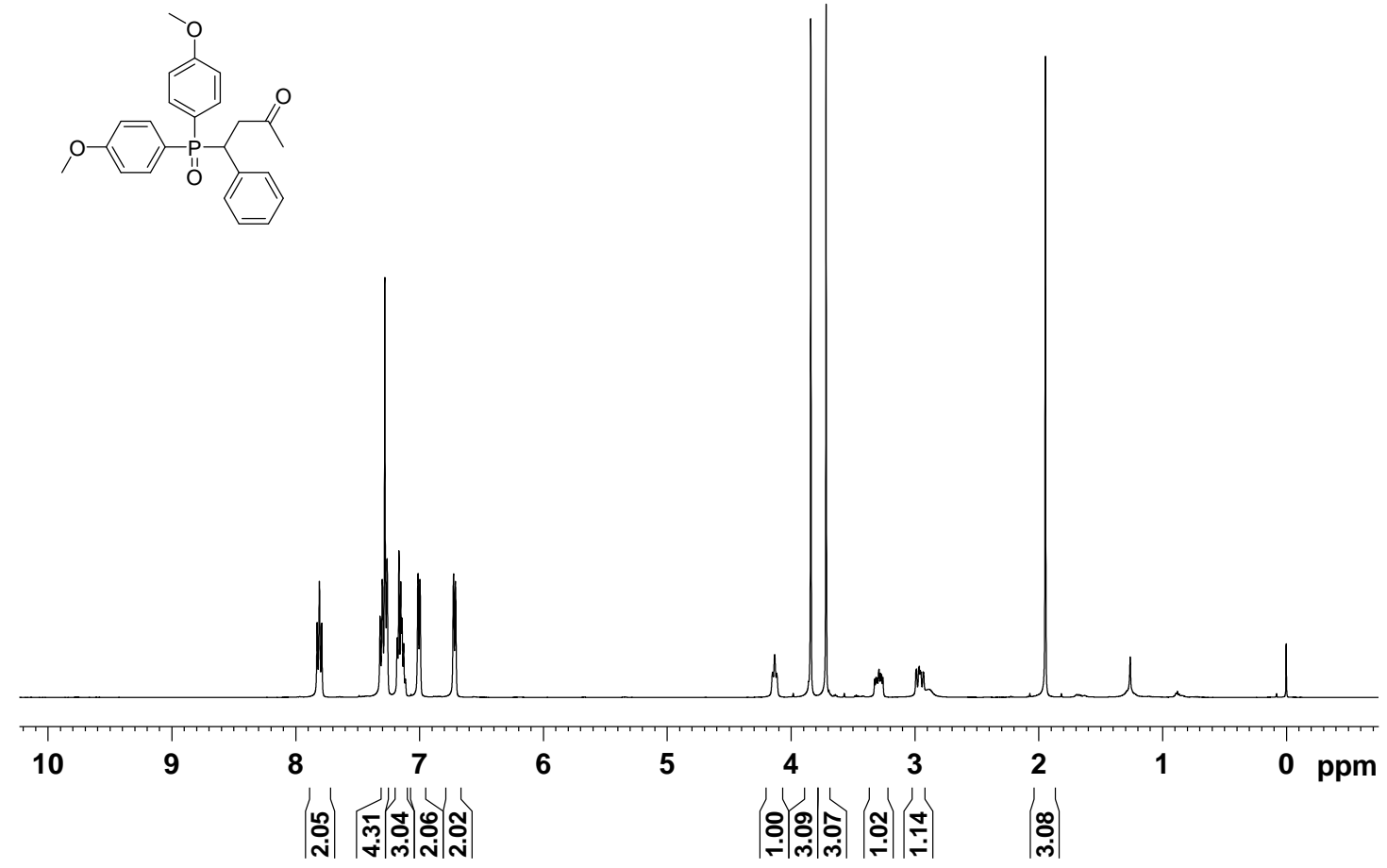


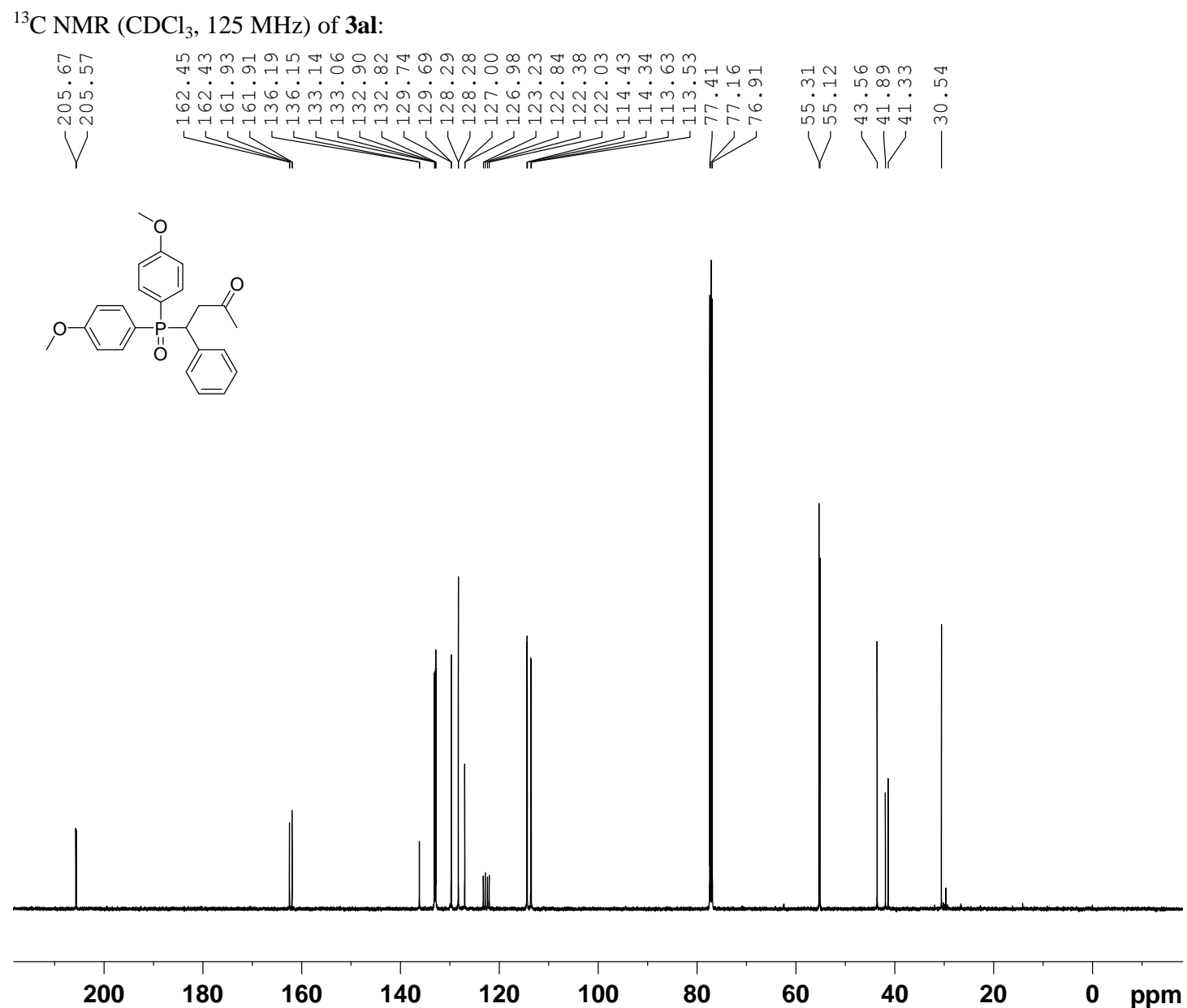

${ }^{31} \mathrm{P}$ NMR $\left(\mathrm{CDCl}_{3}, 202 \mathrm{MHz}\right)$ of 3al:
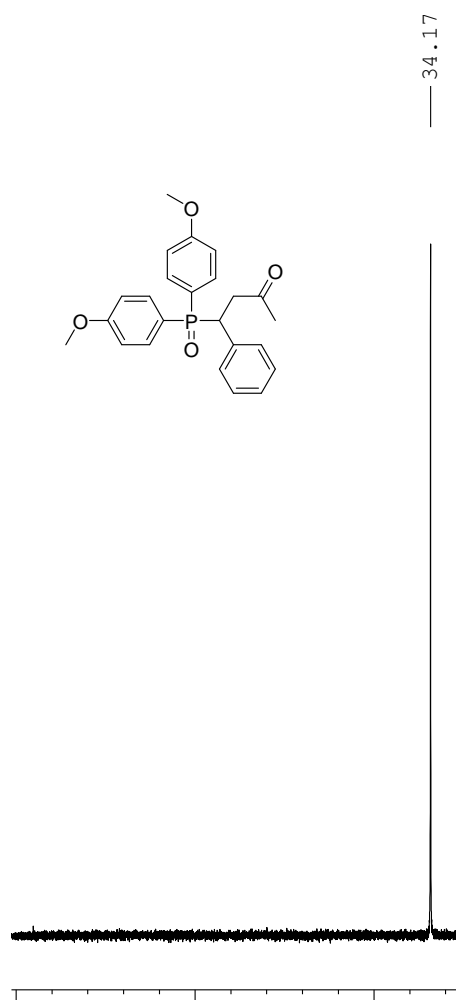
${ }^{1} \mathrm{H} \mathrm{NMR}\left(\mathrm{CDCl}_{3}, 500 \mathrm{MHz}\right)$ of 3am:

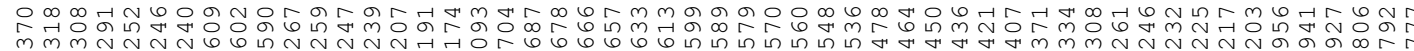

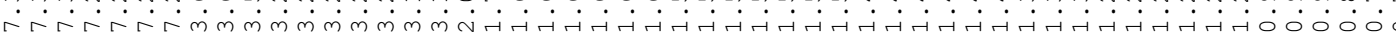

thesing
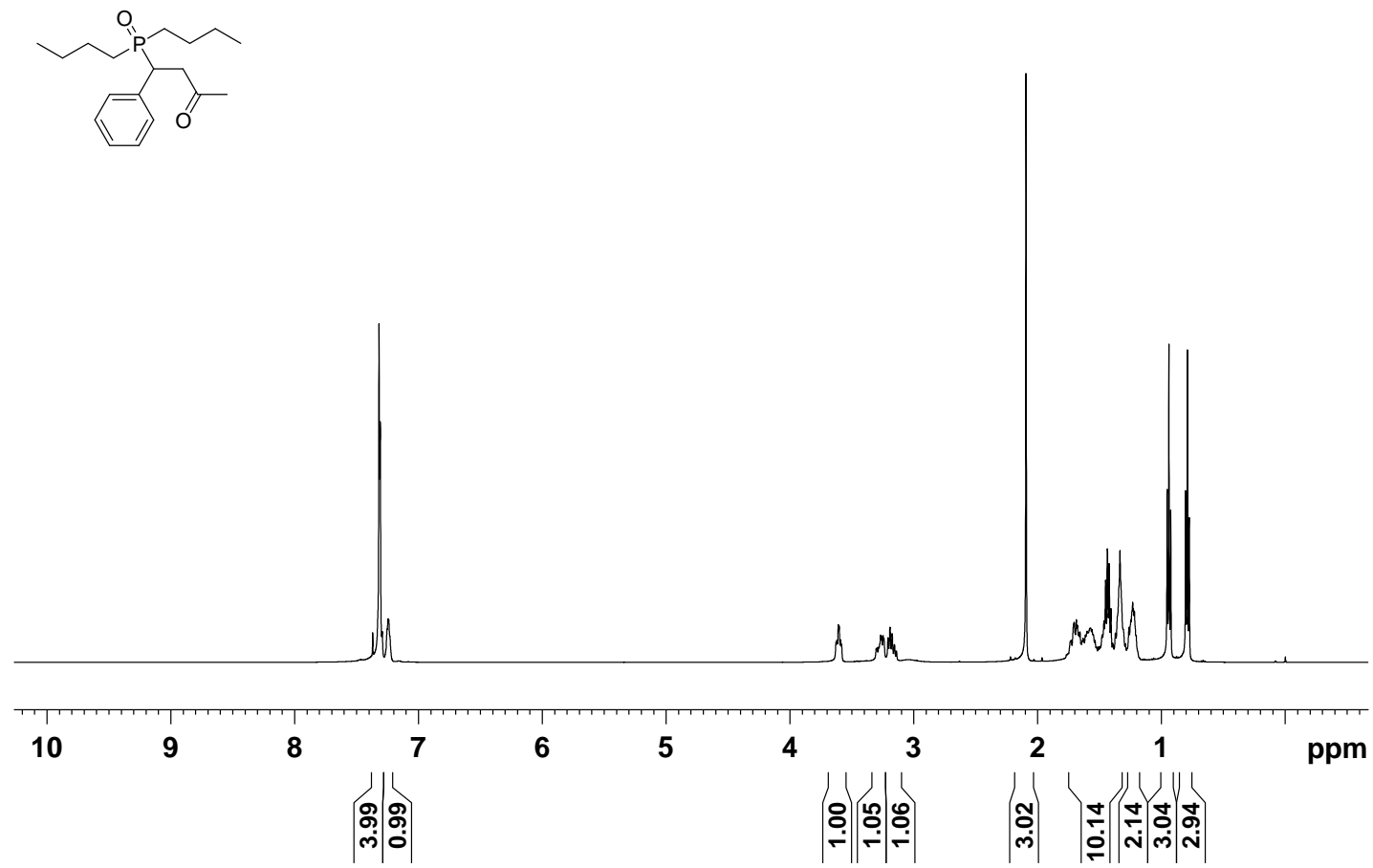

${ }^{13} \mathrm{C} \mathrm{NMR}\left(\mathrm{CDCl}_{3}, 125 \mathrm{MHz}\right)$ of 3am:
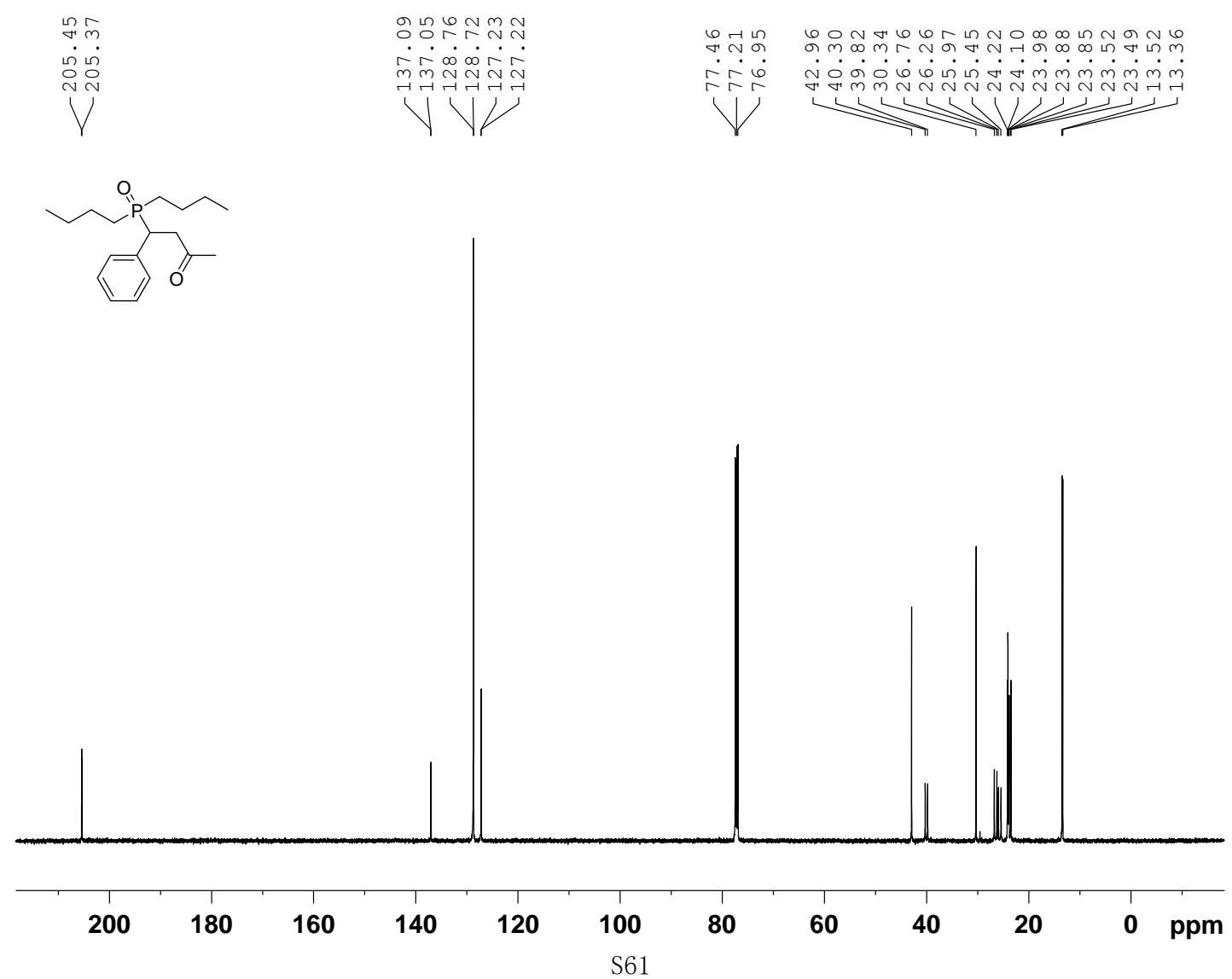
${ }^{31} \mathrm{P}$ NMR $\left(\mathrm{CDCl}_{3}, 202 \mathrm{MHz}\right)$ of 3am:

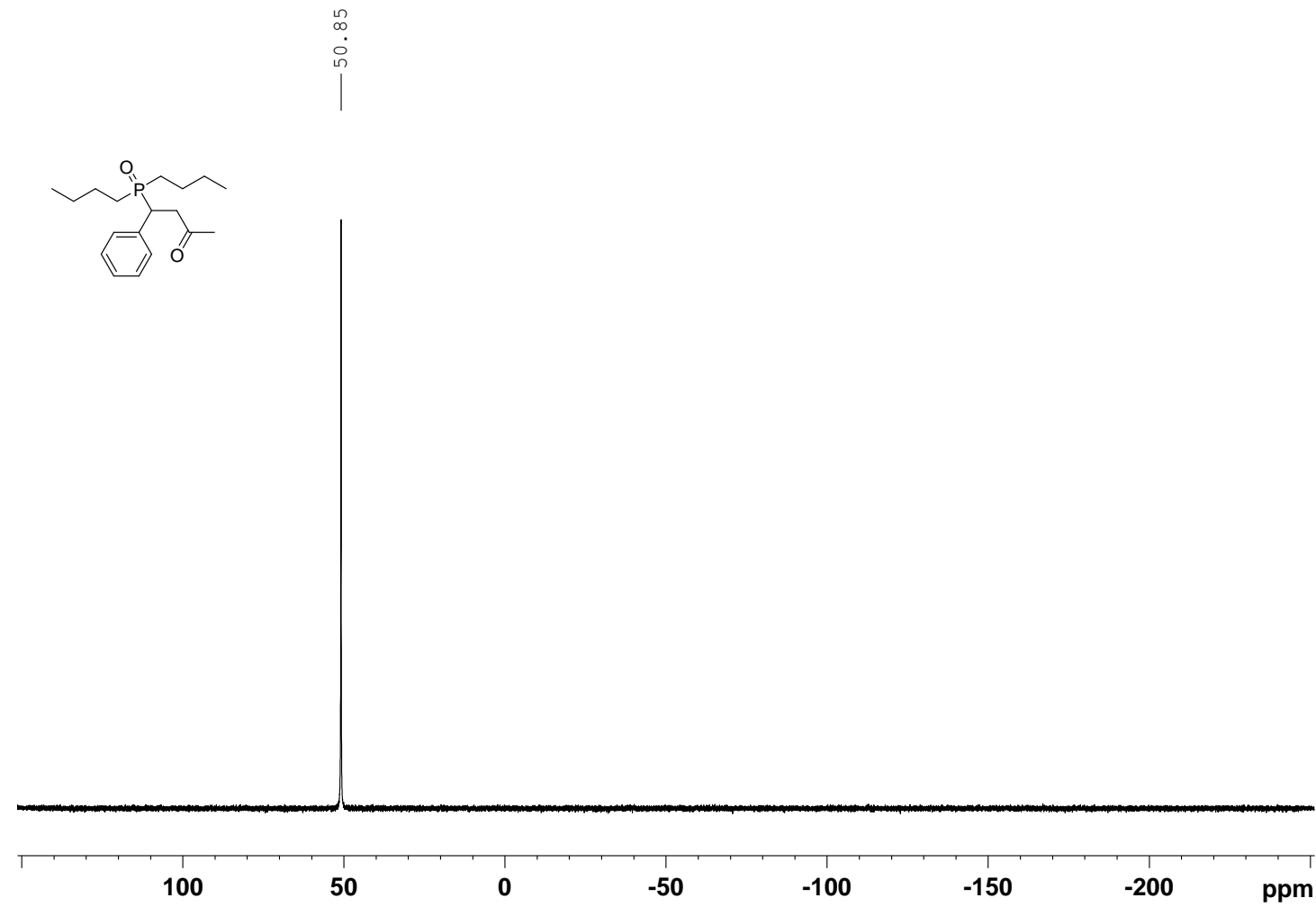

${ }^{1} \mathrm{H} \mathrm{NMR}\left(\mathrm{CDCl}_{3}, 500 \mathrm{MHz}\right)$ of 3an:

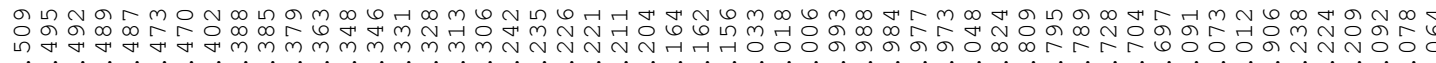

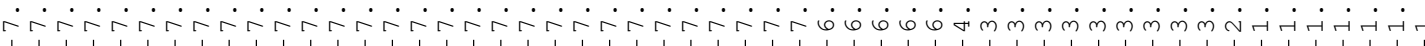

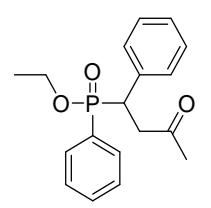

N/IIIII

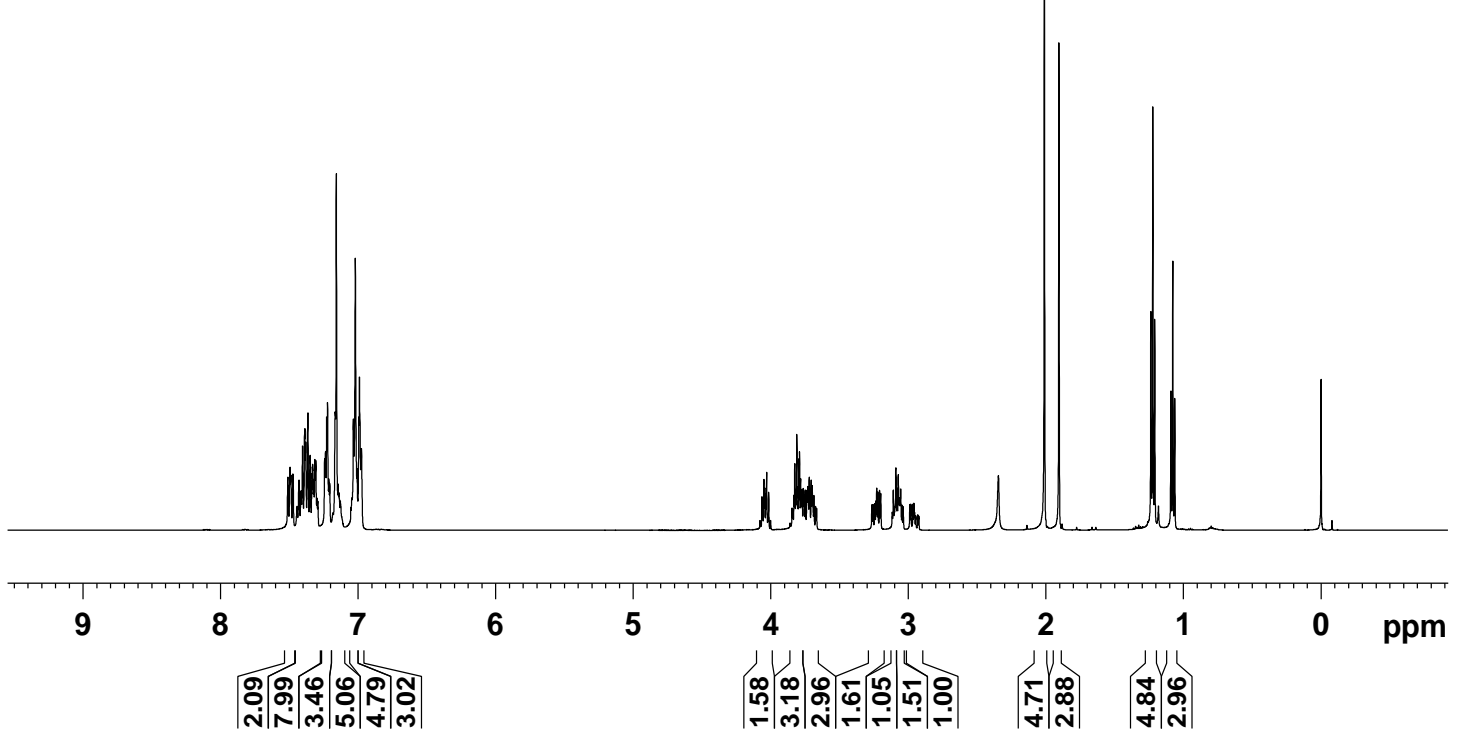


${ }^{13} \mathrm{C}$ NMR $\left(\mathrm{CDCl}_{3}, 125 \mathrm{MHz}\right)$ of 3an:

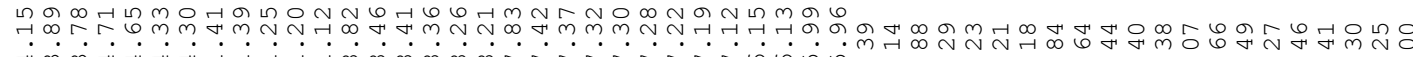

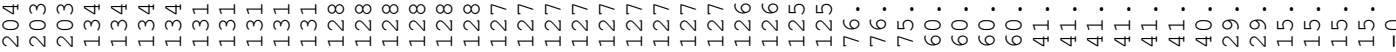

V<smiles>CCOP(=O)(c1ccccc1)C(CC(C)=O)c1ccccc1</smiles>

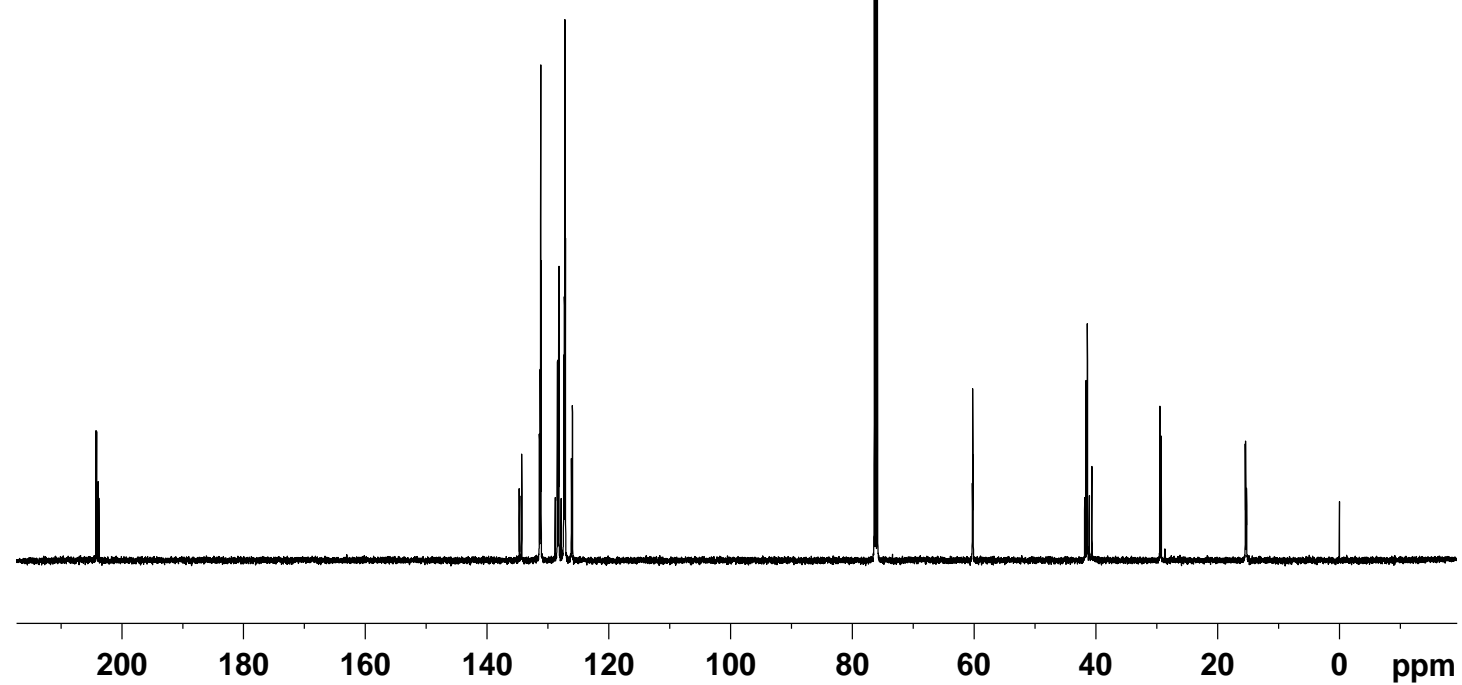

${ }^{31} \mathrm{P}$ NMR $\left(\mathrm{CDCl}_{3}, 202 \mathrm{MHz}\right)$ of $\mathbf{3 a n}:$

ฟै
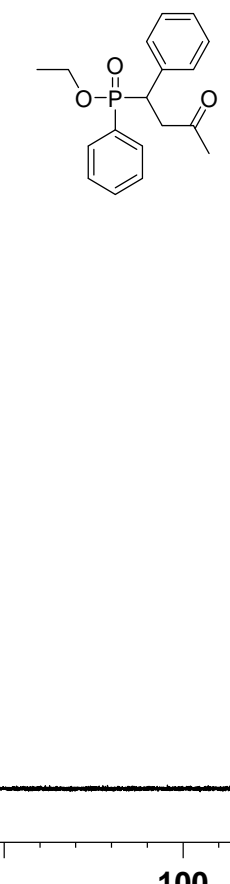

100

50

0

$-50$

$-100$

$-150$

$-200$

ppm 
${ }^{1} \mathrm{H} \mathrm{NMR}\left(\mathrm{CDCl}_{3}, 500 \mathrm{MHz}\right)$ of 3ap:

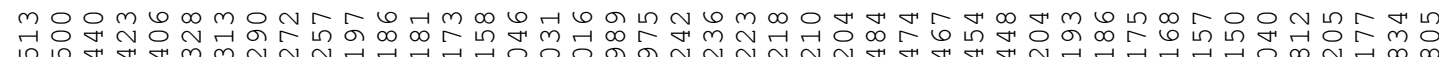
ம. (n)
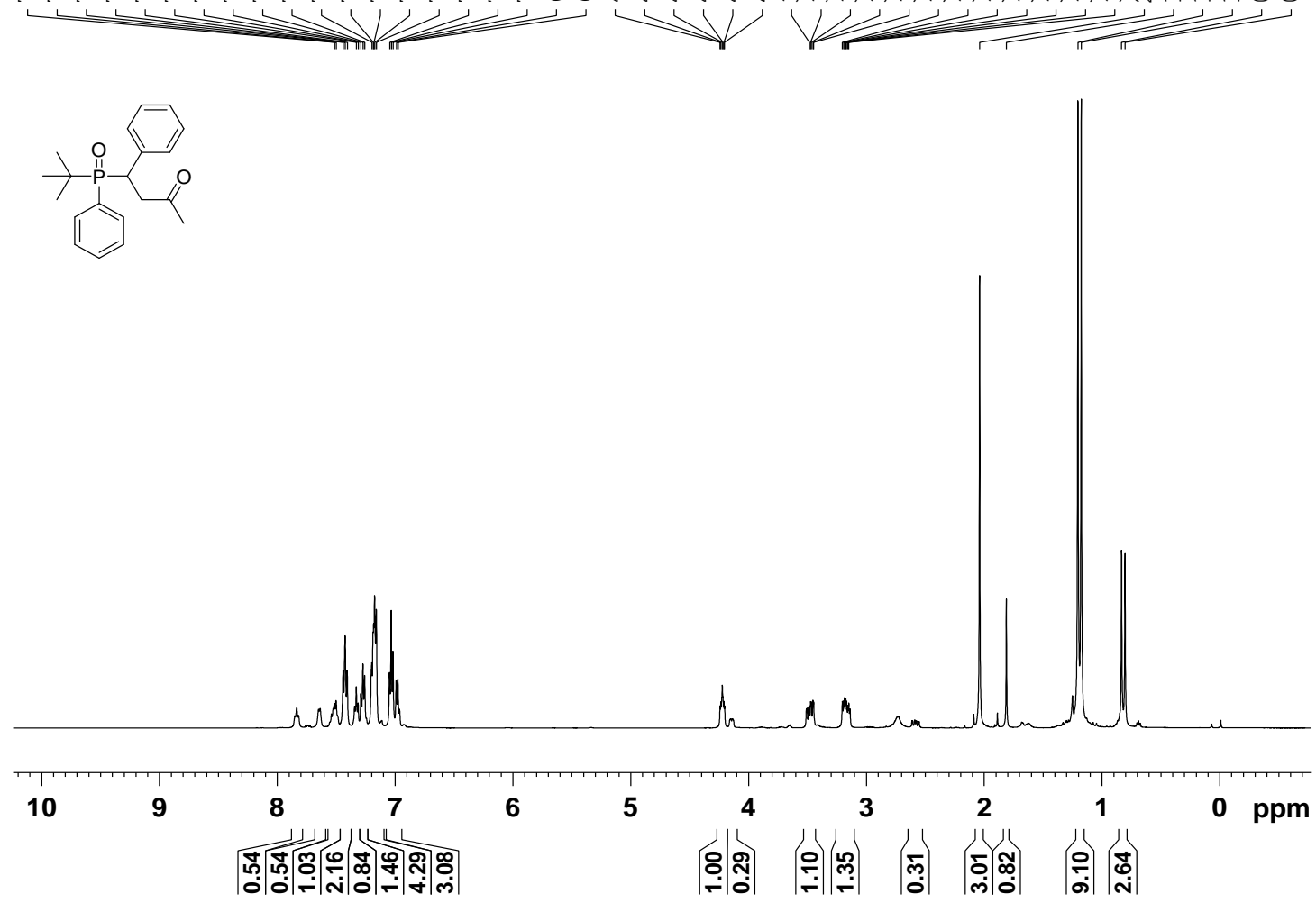

${ }^{13} \mathrm{C} \mathrm{NMR}\left(\mathrm{CDCl}_{3}, 125 \mathrm{MHz}\right)$ of 3ap:

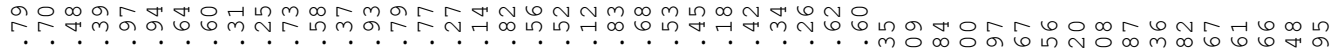

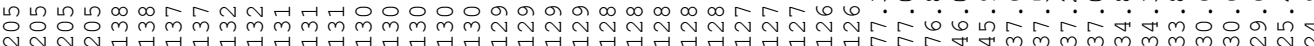

W
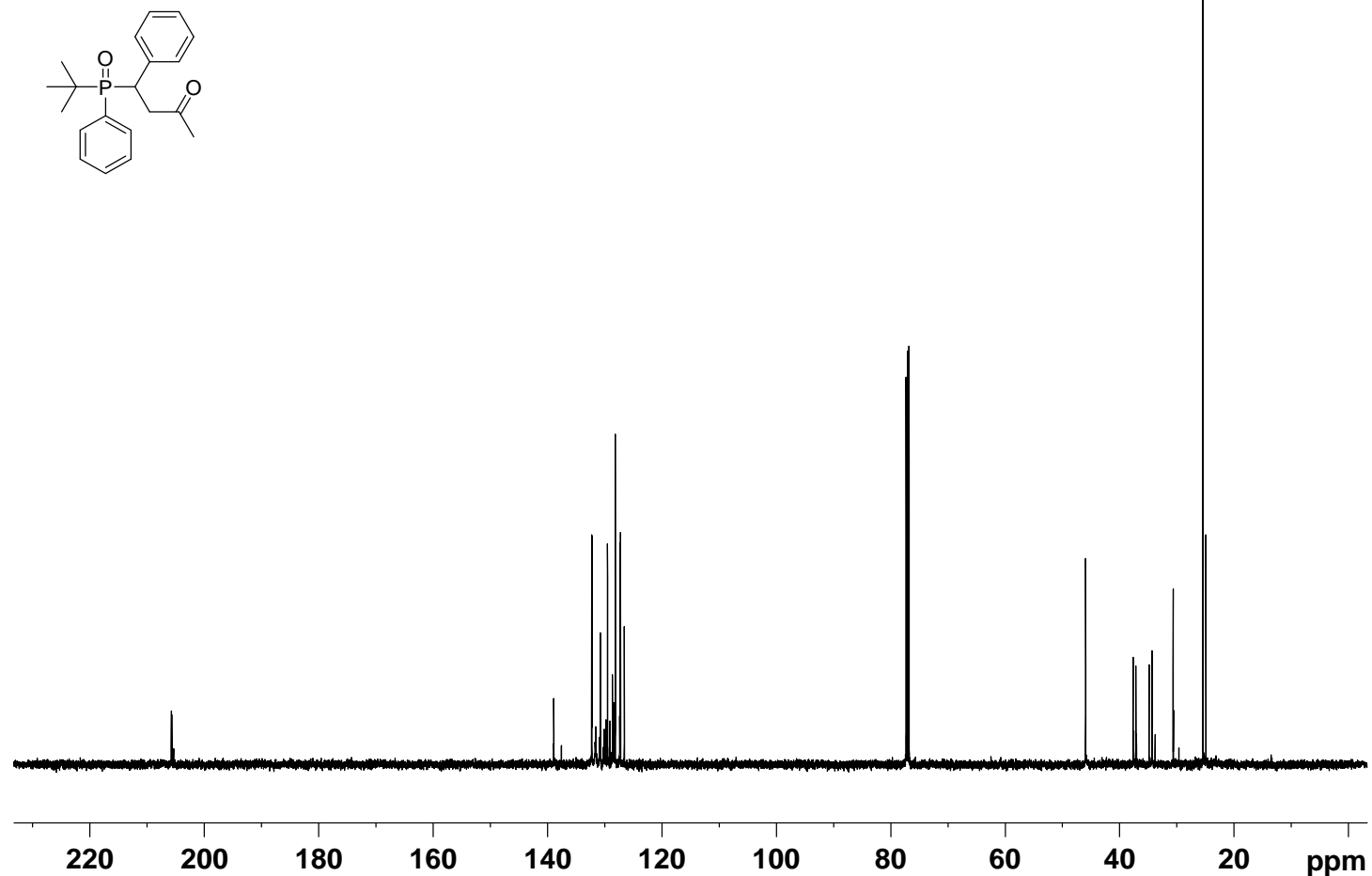
${ }^{31} \mathrm{P}$ NMR $\left(\mathrm{CDCl}_{3}, 202 \mathrm{MHz}\right)$ of 3ap:

Vై

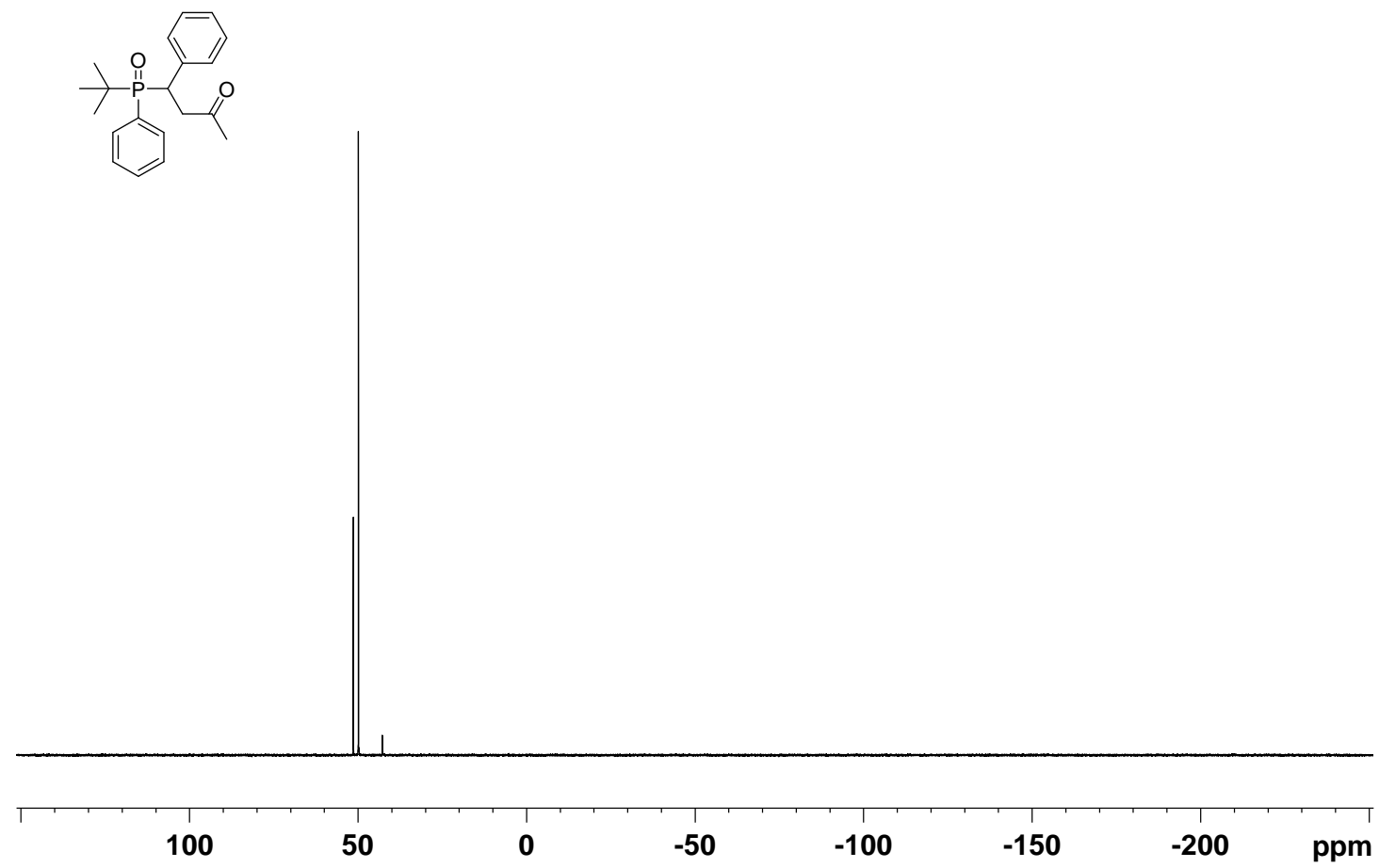

${ }^{1} \mathrm{H} \mathrm{NMR}\left(\mathrm{CDCl}_{3}, 500 \mathrm{MHz}\right)$ of 4 :

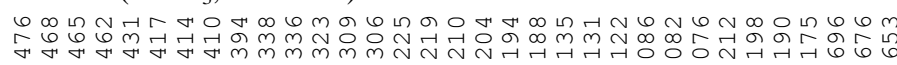

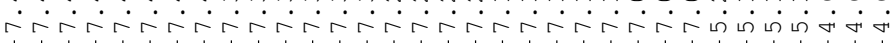

(n)
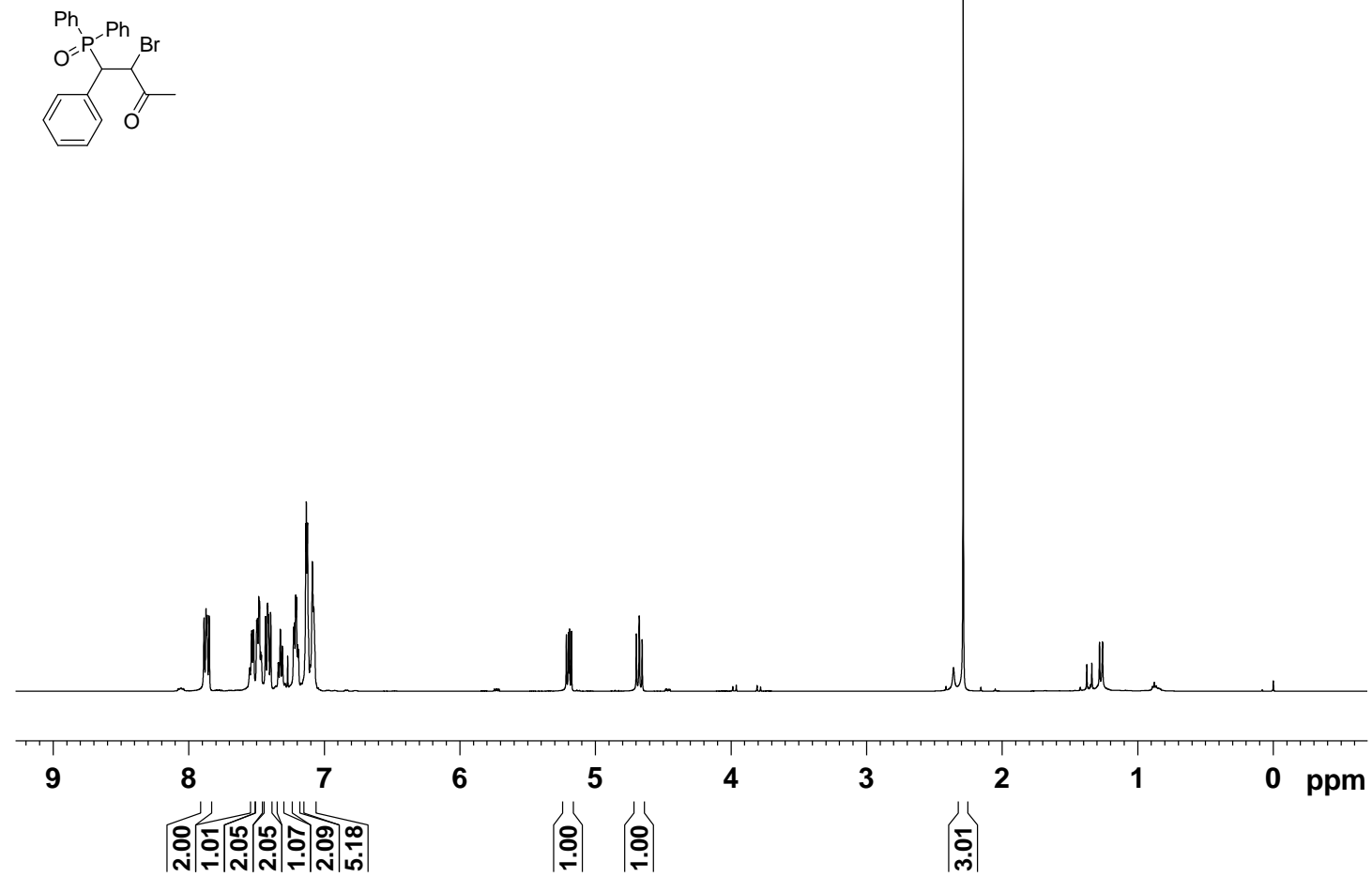
${ }^{13} \mathrm{C} \mathrm{NMR}\left(\mathrm{CDCl}_{3}, 125 \mathrm{MHz}\right)$ of $\mathbf{4}$ :
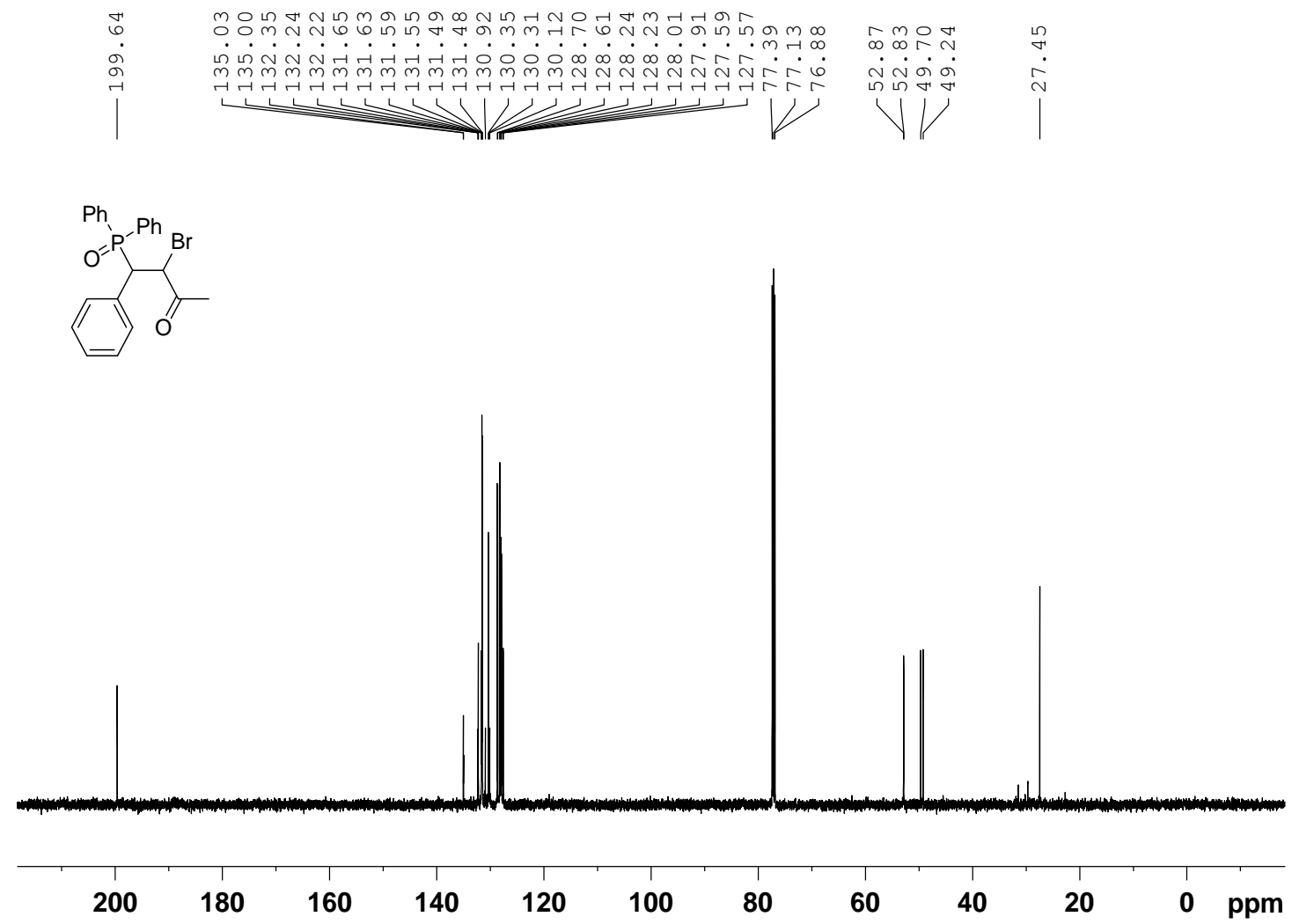

${ }^{31} \mathrm{P} \mathrm{NMR}\left(\mathrm{CDCl}_{3}, 202 \mathrm{MHz}\right)$ of 4 :
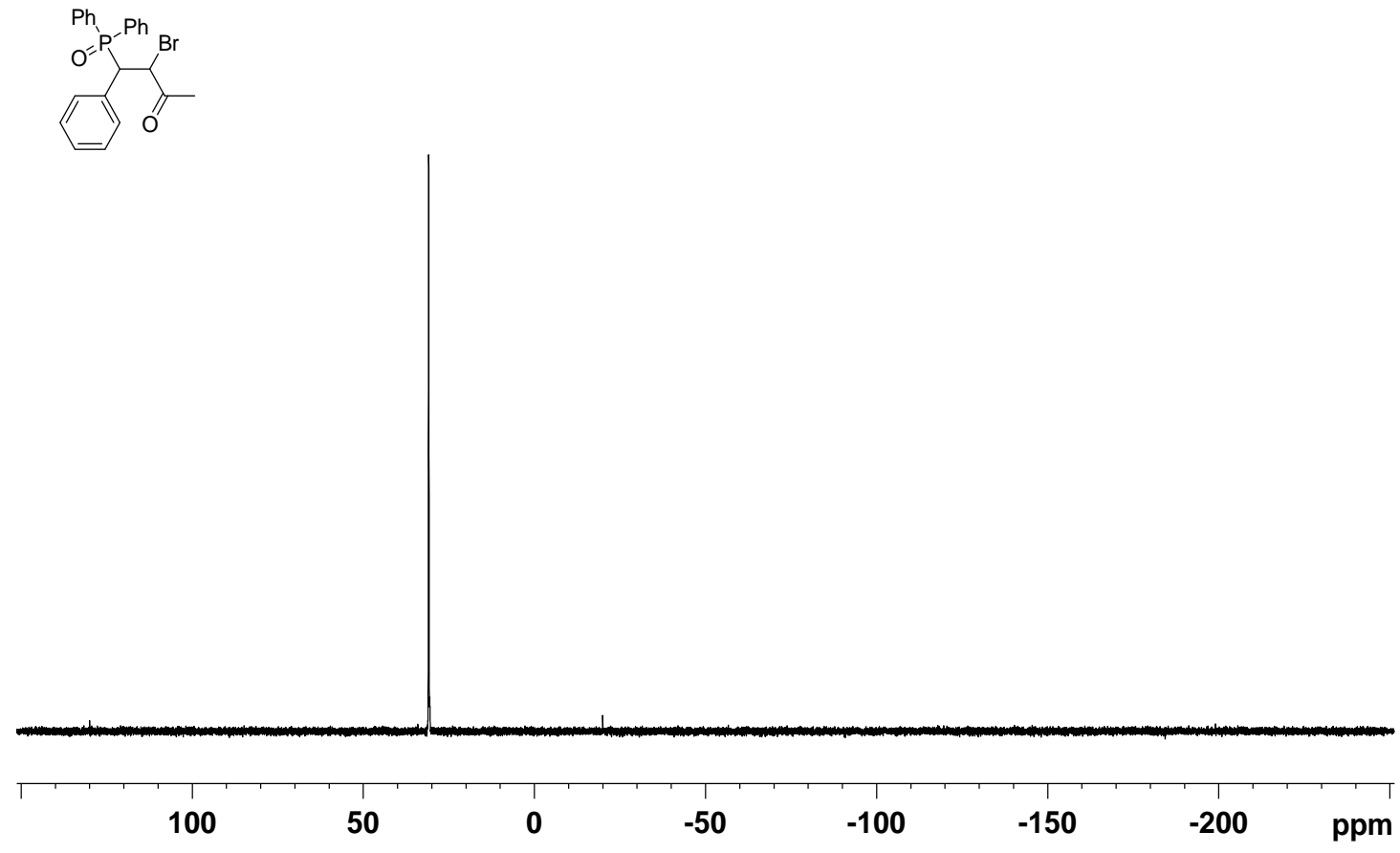
${ }^{1} \mathrm{H}$ NMR $\left(\mathrm{CDCl}_{3}, 500 \mathrm{MHz}\right)$ of $\mathbf{5}$ :

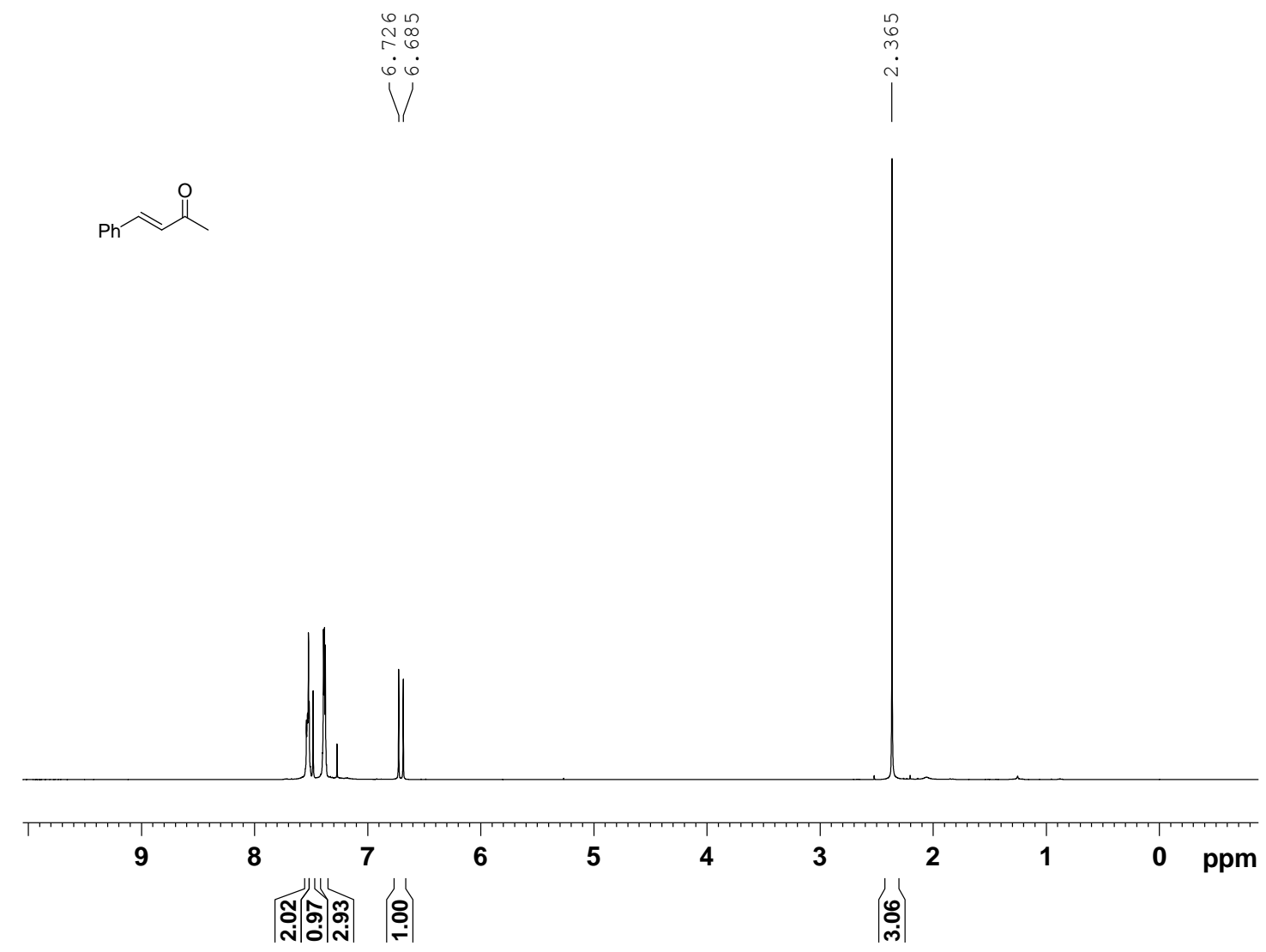

${ }^{13} \mathrm{C} \mathrm{NMR}\left(\mathrm{CDCl}_{3}, 125 \mathrm{MHz}\right)$ of $\mathbf{5}$ :

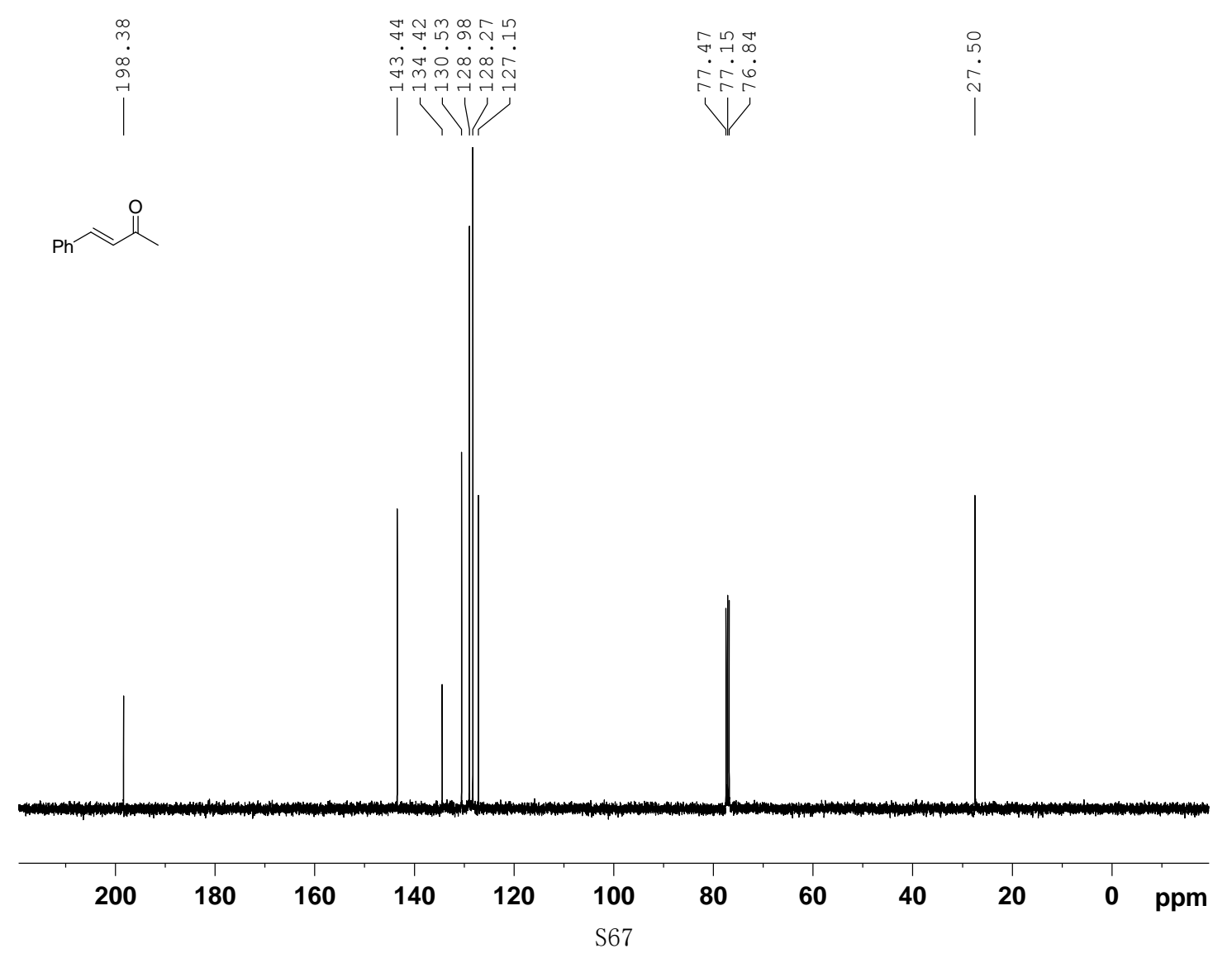

